\title{
Point vortex dynamics as zero-radius limit of the motion of a rigid body in an irrotational fluid
}

\author{
Olivier Glass • Alexandre Munnier • \\ Franck Sueur
}

July 3,2021

\begin{abstract}
The point vortex system is usually considered as an idealized model where the vorticity of an ideal incompressible two-dimensional fluid is concentrated in a finite number of moving points. In the case of a single vortex in an otherwise irrotational ideal fluid occupying a bounded and simply-connected two-dimensional domain the motion is given by the so-called Kirchhoff-Routh velocity which depends only on the domain. The main result of this paper establishes that this dynamics can also be obtained as the limit of the motion of a rigid body immersed in such a fluid when the body shrinks to a massless point particle with fixed circulation. The rigid body is assumed to be only accelerated by the force exerted by the fluid pressure on its boundary, the fluid velocity and pressure being given by the incompressible Euler equations, with zero vorticity. The circulation of the fluid velocity around the particle is conserved as time proceeds according to Kelvin's theorem and gives the strength of the limit point vortex. We also prove that in the different regime where the body shrinks with a fixed mass the limit dynamics is governed by a second-order differential equation involving a Kutta-Joukowski-type lift force.

To prove these results, in a first step we reformulate the dynamics of the body in order to make more explicit different kind of interactions with the fluid. Precisely we establish that the Newton-Euler equations of translational and rotational dynamics of the body can be seen as a 3-dimensional ODE with

O. Glass

CEREMADE, UMR CNRS 7534, Université Paris-Dauphine, Place du Maréchal de Lattre de Tassigny, 75775 Paris Cedex 16, France

\section{A. Munnier}

Université de Lorraine, Institut Elie Cartan de Lorraine, UMR 7502, Nancy-Université, Vandoeuvre-lès-Nancy, F-54506, France \& CNRS, Institut Elie Cartan de Lorraine, UMR 7502, Nancy-Université, Vandoeuvre-lès-Nancy, F-54506, France

\section{F. Sueur}

Institut de Mathématiques de Bordeaux, UMR CNRS 5251, Université de Bordeaux, 351 cours de la Libération, F-33405 Talence Cedex, France.
\end{abstract}


coefficients solving an auxiliary problem for the fluid. When the circulation around the body is zero, this equation is a geodesic equation for a metric associated with the well-known "added inertia" phenomenon; with a nonzero circulation, an additional term similar to the Lorentz force of electromagnetism appears. Then, in the zero-radius limit, surprising relations between leading and subprincipal orders of various terms and modulation variables show up and allow us to establish a normal form with a gyroscopic structure. This leads to uniform estimates on the body's dynamics thanks to a modulated energy, and therefore allows us to describe the transition of the dynamics in the limit.

\section{Contents}

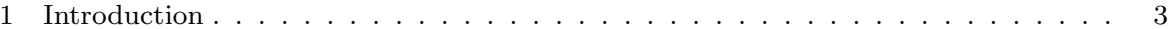

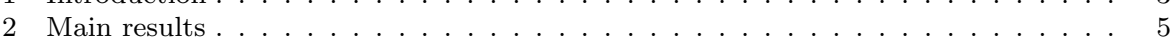

2.1 Dynamics of a solid with fixed size and mass . . . . . . . . . . . . . . . 5

2.2 Case (i): Dynamics of a solid shrinking to a point-mass particle . . . . . . . 15

2.3 Case (ii): Dynamics of a solid shrinking to a massless point particle . . . . . . 17

3 Comments and organization of the paper . . . . . . . . . . . . . . . . . 18

3.1 Comments...................... 18

3.2 Organization of the paper . . . . . . . . . . . . . . . . 19

4 Case without external boundary . . . . . . . . . . . . . . . . . . 20

4.1 Recasting of the system as an ODE . . . . . . . . . . . . . . . . 20

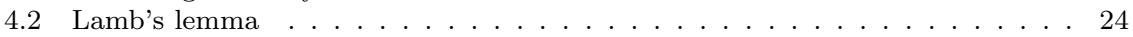

4.3 Proof of Theorem $4.4 \ldots \ldots \ldots \ldots \ldots \ldots$

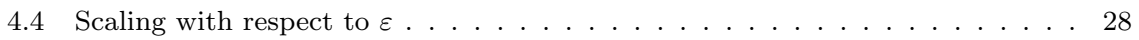

4.5 Case (i) without external boundary . . . . . . . . . . . . . . . . . . . 30

4.6 Case (ii) without external boundary . . . . . . . . . . . . . . . . 31

5 Recasting the system: Proofs of Theorem 2.2 Lemma 2.5 Proposition 2.6 Propo-

sition 2.8 and Theorem 2.9 . . . . . . . . . . . . . . . . . . . 32

5.1 Splitting the proof of Theorem $2.2 \ldots \ldots \ldots \ldots$. . . . . . . . . . . . . . . . . . .

5.2 Reformulation of the potential part: Proof of Lemma 5.1. . . . . . . . . . 33

5.3 Reformulation of the cross part: Proof of Lemma 5.3 . . . . . . . . . . . 37

5.4 Decomposition of the Christoffel symbols: Proof of Proposition 2.8 . . . . . 38

5.5 Identification of the electric potential: Proof of Lemma 2.5 . . . . . . . . . . 43

5.6 Conservation of energy: Proof of Proposition 2.6 . . . . . . . . . . . . 44

5.7 The case of a disk: proof of Theorem 2.9 . . . . . . . . . . . . . . . . . . . 45

6 Convergence to the massive point vortex system in Case (i): Proof of Theorem 2.11 46

6.1 Normal form . . . . . . . . 47

6.2 Renormalized energy estimates . . . . . . . . . . . . . . . . . . . . . . . . . . 48

6.3 Passage to the limit . . . . . . . . . . . . . . . . . . . . . . . . . . 49

7 Convergence to the point vortex system in Case (ii): Proof of Theorem 2.12 . . . . 51

7.1 The case of a homogeneous disk . . . . . . . . . . . . . . . 51

7.2 Geodesic-gyroscopic normal form . . . . . . . . . . . . . . . . . 54

7.3 Modulated energy estimates . . . . . . . . . . . . . . . . . . 56

7.4 Passage to the limit . . . . . . . . . . . . . . . . . . . . . . . . 58

7.5 The case of a non-homogeneous disk . . . . . . . . . . . . . . . . . . . . . 58

8 Asymptotic development of the stream and potential functions . . . . . . . . . . . 60

8.1 A few reminders about single-layer potentials . . . . . . . . . . . . . 60

8.2 Statements of the results . . . . . . . . . . . . . 64 64

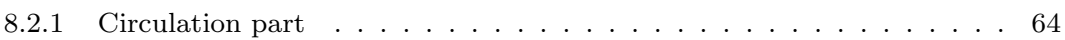

8.2 .2 Potential part . . . . . . . . . . . . . 66

8.3 Asymptotic expansion of the circulation part: Proof of Proposition 8.4 and of

Lemma 6.4 . . . . . . . . . . . . . . . . . . . . . . . 68

8.3.1 First Step. Reduction to integral equations . . . . . . . . . . . 68 
8.3.2 Second Step. Construction of an approximate solution . . . . . . . . 70

8.3.3 Third Step. Existence and estimate of the remainders . . . . . . . . . 74

8.3.4 Fourth Step. Conclusion . . . . . . . . . . . . . . . . . 77

8.4 Asymptotic expansion of the potential part: Proof of Proposition 8.5 . . . . . 78

9 Proof of the normal forms . . . . . . . . . . . . . . . 80

9.1 Asymptotic expansion of the added inertia and the Christoffel symbols . . . . 80

9.2 Asymptotic expansion of $E_{\varepsilon} \ldots \ldots$. . . . . . . . . . . . . . . . . 83

9.3 Asymptotic expansion of $B_{\varepsilon} \ldots \ldots$. . . . . . . . . . . . . . . . . . . . . . . . 90

9.4 End of the proof of the normal forms . . . . . . . . . . . . . . . . . . . . . . 95

\section{Introduction}

The point vortex system is a classical topic which originates from fluid mechanics and goes back to Helmholtz [16], Kirchhoff [22, Poincaré [36], Routh 39], Kelvin [21, and Lin [24,25]. It appeared as an idealized model where the vorticity of an ideal incompressible two-dimensional fluid is concentrated in a finite number of points. Although it does not constitute a solution to the Euler equations in the sense of distributions, it is now understood that point vortices can be viewed as limits of concentrated smooth vortices which evolve according to the Euler equations. In the case of a single vortex moving in a bounded and simply-connected domain this was proved by Turkington in 41]. An extension to the case of several vortices was given by Marchioro and Pulvirenti; see [27. Recently Gallay has proven in [9] that the point vortex system can also be obtained as vanishing viscosity limits of concentrated smooth vortices evolving according to the incompressible Navier-Stokes equations.

The main goal of this paper is to prove that the point vortex system can also be viewed as the limit of the dynamics of a solid, shrinking into a massless point particle with fixed circulation, in free motion in an irrotational bounded flow. By free motion we mean that the rigid body is only accelerated by the force exerted by the fluid pressure on its boundary, the fluid velocity and pressure being given by the incompressible Euler equations with zero vorticity. In a different regime, we also derive a different "massive" point vortex system evoked (in the case of two point vortices in the whole plane) by Friedrichs in [8. Chapter 3] under the terminology of bound vortices (as opposed to free vortices). In this case the dynamics is given by a second-order differential equation involving a gyroscopic force similar to the celebrated Kutta-Joukowski-type lift force revealed in the case of a single body in a irrotational unbounded flow at the beginning of the 20th century during the first mathematical investigations in aeronautics; see for example [23]. This result extends the one obtained in 11 to the case where the solid-fluid system is bounded.

To distinguish these two limits of the dynamics of the solid when its size goes to 0 we therefore introduce two cases: Case (i) when the mass of the solid is fixed (and then the solid tends to a point-mass particle), and Case (ii): when the mass tends to 0 along with the size (and then the solid tends to a massless point particle). In particular Case (ii) encompasses the case of fixed density. The main results in this paper establish the massive point vortex system in Case (i), see Theorem 2.11, and the classical point vortex system in Case (ii), 
see Theorem 2.12, as limits of the dynamics of a shrinking solid in a fluid in a cavity. In both cases the strength of the point vortex obtained in the limit is given by the circulation around the body. This circulation is supposed held fixed independently of the size of the body and is conserved as time proceeds according to Kelvin's theorem.

From the fluid viewpoint the circulation around the body somehow encodes the amount of vorticity hidden in the body. The limit where the body has a diameter tending to zero therefore corresponds to a singular perturbation problem (in space). Indeed it is well understood since the work [19, see also [26], that when a solid obstacle with a nonzero given circulation is held fixed in a perfect incompressible fluid, with possibly nonzero vorticity, then in the limit the obstacle shrinks into a fixed point particle and the Euler equation driving the fluid evolution has to be modified: in the Biot-Savart law providing the fluid velocity generated by the fluid vorticity, a point vortex placed at the fixed position of the point obstacle has to be added to the fluid vorticity, with a strength equal to the circulation previously mentioned.

Still the dynamics of an immersed rigid body requires a more precise analysis, in particular because it is driven by the fluid pressure on the boundary of the solid, a quantity which depends in a nonlinear and non local way on the fluid velocity and hides a remote interaction between the moving body and the exterior boundary. Moreover, in the zero-radius limit, the pressure field on the boundary of the solid is expected to be singular and the Newton-Euler equations driving the particle's dynamics involve a singular perturbation problem in time (in addition to the singularity in space), in a particularly intricate way for asymmetric particles (actually for any other form than a disk) and even more so for light particles whose mass and moment of inertia go to zero (Case (ii)). Our analysis relies on a detailed treatment of the structure of these singularities, first for any positive body radius and then for vanishingly small radius, to describe the transition of the dynamics in the limit.

In a first step, see Theorem 2.2 below, we reformulate the dynamics of the body in order to make more explicit different kind of interactions with the fluid. Indeed the fluid velocity can be recovered from the solid position and velocity by an elliptic-type problem, so that the fluid state may be seen as solving an auxiliary steady problem, where time only appears as a parameter, instead of the unsteady incompressible Euler equation. We establish a reformulation of the Newton-Euler equations as a second-order differential equation on the solid position which is determined by three degrees of freedom (two for the translation and one for the rotation) with coefficients obtained by solving the auxiliary fluid problem. Indeed we establish that the dynamics of the body may be recast as a geodesic equation with an applied force similar to the Lorentz force of electromagnetism. The metric associated with the geodesic part of the equation is given by the total inertia, that is the inertia of the solid to which one adds the so-called "added inertia": a symmetric nonnegative matrix depending only on the body's shape and position, encoding the amount of incompressible fluid that the rigid body has also to accelerate around itself. The magnetic part of the Lorentz force is a gyroscopic force, proportional to the circulation 
around the body, which can be seen as an extension of the Kutta-Joukowski lift force. In particular the contribution of this force to the energy variation vanishes. On the contrary, the electric part of the Lorentz force leads to an energy exchange between the fluid and the solid.

To be able to describe the transition of the dynamics in the zero-radius limit we would like to deduce uniform estimates from this geodesic-Lorentz reformulation in order to pass to the limit. Still the equation contains also the electric-type force which prevents from obtaining such bounds from an energy estimate. Indeed even if the whole system is hamiltonian, see [13, nothing excludes a priori some sharp energy exchange between the fluid and the immersed rigid particles. To overcome this difficulty the second part of our analysis exploits the structure of the various terms of the geodesic-Lorentz reformulation in the limit where the size of the solid goes to 0 . These terms involve integrals of functions describing the part of the fluid velocity due to the body's velocity and to the body's circulation. These functions are given as solutions to some elliptic-type problems in a domain which is the complement to the vanishingly small body in the cavity, which entails some small-scale variations of these functions. Multi-scale expansions allow us to precisely describe their asymptotic behaviour and to deduce asymptotic expansions of every term of the geodesic-Lorentz reformulation. In Case (i) a rough expansion is sufficient as the only leading term is gyroscopic and therefore allows to obtain some uniform estimates. In Case (ii) a few striking combinations allow us to transfer the bad electric-type term as a modulation of the particle velocity appearing in the other terms of the equation (i.e. the geodesic and magnetic terms). This leads to a geodesic-gyroscopic asymptotic normal form of the Newton-Euler equations where a modulation of the unknown is used. This normal form is tailored to obtain uniform estimates on the dynamics thanks to some energytype quantities modulated by the limit dynamics. These uniform estimates then allow us to pass to the limit in both cases.

\section{Main results}

\subsection{Dynamics of a solid with fixed size and mass}

To begin with, let us recall the dynamics of a solid with fixed size and mass in a perfect incompressible fluid. We denote by $\Omega$ the bounded open smooth and simply connected ${ }^{1}$ domain of $\mathbb{R}^{2}$ occupied by the fluid-solid system. At the initial time, the domain of the solid is a non-empty closed smooth and simply connected domain $\mathcal{S}_{0} \subset \Omega$ and $\mathcal{F}_{0}:=\Omega \backslash \mathcal{S}_{0}$ is the domain of the fluid. There is no loss of generality in assuming that the center of mass of the solid coincides at the initial time with the origin.

\footnotetext{
1 the simple connectedness is a simplifying assumption but is actually not essential in the analysis.
} 
The rigid motion of the solid is represented at every moment by a rotation matrix

$$
R(\vartheta(t)):=\left[\begin{array}{cc}
\cos \vartheta(t) & -\sin \vartheta(t) \\
\sin \vartheta(t) & \cos \vartheta(t)
\end{array}\right],
$$

describing the rotation of the solid with respect to its original position and a vector $h(t)$ in $\mathbb{R}^{2}$ describing the position of the center of mass. The domain of the solid at every time $t>0$ is therefore $\mathcal{S}(t):=R(\vartheta(t)) \mathcal{S}_{0}+h(t)$, while the domain of the fluid is $\mathcal{F}(t):=\Omega \backslash \mathcal{S}(t)$ (see Fig. 1). The fluid-solid system is

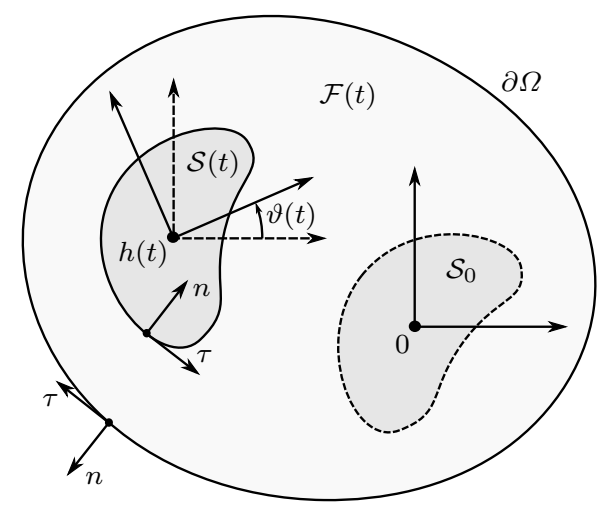

Fig. 1 The domains $\Omega, \mathcal{S}(t)$ and $\mathcal{F}(t):=\Omega \backslash \overline{\mathcal{S}(t)}$ of the problem.

governed by the following set of coupled equations:

Fluid equations:

$$
\begin{array}{rlrl}
\frac{\partial u}{\partial t}+(u \cdot \nabla) u+\nabla \Pi=0 & & \text { in } \mathcal{F}(t) \\
\operatorname{div} u=0 & \text { in } \mathcal{F}(t) .
\end{array}
$$

Solid equations:

$$
\begin{aligned}
m h^{\prime \prime} & =\int_{\partial \mathcal{S}(t)} \Pi n \mathrm{~d} s, \\
\mathcal{J} \vartheta^{\prime \prime} & =\int_{\partial \mathcal{S}(t)}(x-h(t))^{\perp} \cdot \Pi n \mathrm{~d} s .
\end{aligned}
$$

Boundary conditions:

$$
\begin{aligned}
& u \cdot n=\left(\vartheta^{\prime}(\cdot-h)^{\perp}+h^{\prime}\right) \cdot n \quad \text { on } \partial \mathcal{S}(t), \\
& u \cdot n=0 \quad \text { on } \partial \Omega \text {. }
\end{aligned}
$$

Above $u$ and $\Pi$ denote the velocity and pressure fields in the fluid, $m>0$ and $\mathcal{J}>0$ denote respectively the mass and the moment of inertia of the body while the fluid is supposed to be homogeneous of density 1 , to simplify the notations. When $x=\left(x_{1}, x_{2}\right)$ the notation $x^{\perp}$ stands for $x^{\perp}=\left(-x_{2}, x_{1}\right)$, $n$ denotes the unit normal vector pointing outside of the fluid. Let us also 
emphasize that $\mathrm{d} s$ will stand for the arc length without any distinction between $\partial \Omega, \partial \mathcal{S}(t)$ and $\partial \mathcal{S}_{0}$.

In this paper, we consider irrotational solutions, that is, solutions satisfying

$$
\operatorname{curl} u(t, \cdot)=0 \text { in } \mathcal{F}(t) .
$$

Actually introducing the initial data:

$$
\begin{gathered}
u_{\mid t=0}=u_{0} \text { in } \mathcal{F}_{0}, \\
\vartheta(0)=0, h(0)=0, h^{\prime}(0)=\ell_{0}, \vartheta^{\prime}(0)=\omega_{0},
\end{gathered}
$$

the condition $(2.2)$ merely depends on the initial vorticity $\operatorname{curl} u_{0}$ : if the flow is irrotational at the initial time, that is curl $u_{0}=0$ in $\mathcal{F}_{0}$, it will remain irrotational for every time as in (2.2), according to Helmholtz's third theorem. On the other hand the circulation around the body is constant in time:

$$
\int_{\partial \mathcal{S}(t)} u(t) \cdot \tau \mathrm{d} s=\gamma
$$

with

$$
\gamma:=\int_{\partial \mathcal{S}_{0}} u_{0} \cdot \tau \mathrm{d} s,
$$

according to Kelvin's theorem. Here $\tau$ denotes the unit counterclockwise tangential vector so that $n=\tau^{\perp}$. Let us mention here that we will also use the notation $\tau$ on $\partial \Omega$ such that $n:=\tau^{\perp}$ so that it is clockwise in this case (see Fig. 1).

In the irrotational case, System 2.1)-2.2 can be recast as an ODE whose unknowns are the degrees of freedom of the solid, namely $\vartheta$ and $h$. In particular, given (2.4), the motion of the fluid is completely determined by the solid position and velocity. To state this rigorously, let us introduce the variables

$$
h:=\left(h_{1}, h_{2}\right) \quad \text { and } \quad q:=\left(\vartheta, h_{1}, h_{2}\right) \in \mathbb{R}^{3} .
$$

Since the domains $\mathcal{S}(t)$ and $\mathcal{F}(t)$ depend on $q$ only, we will rather denote them $\mathcal{S}(q)$ and $\mathcal{F}(q)$ in the rest of the paper. Since throughout this paper we will not consider any collision, we introduce:

$$
\mathcal{Q}:=\left\{q \in \mathbb{R}^{3}: d(\mathcal{S}(q), \partial \Omega)>0\right\}
$$

where $d(A, B)$ denotes the minimal distance between the two sets $A$ and $B$ in the plane

$$
d(A, B):=\inf \left\{|x-y|_{\mathbb{R}^{2}}, x \in A, y \in B\right\} .
$$

Above and throughout the paper we use the notation $|\cdot|_{\mathbb{R}^{d}}$ for the Euclidean norm in $\mathbb{R}^{d}$. Since $\mathcal{S}_{0}$ is a closed subset in the open set $\Omega$, the initial position $q(0)=0$ of the solid belongs to $\mathcal{Q}$. 
Now we need to introduce various objects depending on the geometry and on the constants $m, \mathcal{J}, \gamma$, in order to make the aforementioned ODE explicit.

Kirchhoff potentials $\varphi_{j}$. Consider the functions $\xi_{j}$, for $j=1,2,3$, defined for $(q, x)$ in $\cup_{q \in \mathcal{Q}}(\{q\} \times \mathcal{F}(q))$, by the formula

$$
\xi_{1}(q, x):=(x-h)^{\perp} \text { and } \xi_{j}(q, x):=e_{j-1}, \text { for } j=2,3,
$$

where $e_{1}$ and $e_{2}$ are the unit vectors of the canonical basis. For any $j=1,2,3$, for any $q$ in $\mathcal{Q}$, we denote by $K_{j}(q, \cdot)$ the normal trace of $\xi_{j}$ on $\partial \Omega \cup \partial \mathcal{S}(q)$, that is:

$$
K_{j}(q, \cdot):=n \cdot \xi_{j}(q, \cdot) \text { on } \partial \Omega \cup \partial \mathcal{S}(q) .
$$

Now $q$ being fixed in $\mathcal{Q}$, we introduce the Kirchhoff potentials $\varphi_{j}(q, \cdot)$, for $j=1,2,3$, as the unique (up to an additive constant) solutions in $\mathcal{F}(q)$ of the following Neumann problem:

$$
\begin{array}{ll}
\Delta \varphi_{j}(q, \cdot)=0 & \text { in } \mathcal{F}(q), \\
\frac{\partial \varphi_{j}}{\partial n}(q, \cdot)=K_{j}(q, \cdot) & \text { on } \partial \mathcal{S}(q), \\
\frac{\partial \varphi_{j}}{\partial n}(q, \cdot)=0 & \text { on } \partial \Omega .
\end{array}
$$

We concatenate the $K_{j}$ and $\varphi_{j}$ into the vectors:

$$
\begin{aligned}
\boldsymbol{K}(q, \cdot) & :=\left(K_{1}(q, \cdot), K_{2}(q, \cdot), K_{3}(q, \cdot)\right)^{t} \text { and } \\
\boldsymbol{\varphi}(q, \cdot) & :=\left(\varphi_{1}(q, \cdot), \varphi_{2}(q, \cdot), \varphi_{3}(q, \cdot)\right)^{t},
\end{aligned}
$$

where the exponent $t$ denotes the transpose of the vector.

Stream function $\psi$ for the circulation term. For every $q$ in $\mathcal{Q}$, there exists a unique $C(q) \in \mathbb{R}$ such that the unique solution $\psi(q, \cdot)$ of the Dirichlet problem:

$$
\begin{aligned}
\Delta \psi(q, \cdot) & =0 & & \text { in } \mathcal{F}(q) \\
\psi(q, \cdot) & =C(q) & & \text { on } \partial \mathcal{S}(q) \\
\psi(q, \cdot) & =0 & & \text { on } \partial \Omega,
\end{aligned}
$$

satisfies

$$
\int_{\partial \mathcal{S}(q)} \frac{\partial \psi}{\partial n}(q, \cdot) \mathrm{d} s=-1 .
$$

This can be seen easily by defining the corresponding harmonic function $\tilde{\psi}(q, \cdot)$ with $\tilde{\psi}(q, \cdot)=-1$ on $\partial \mathcal{S}(q)$ and $\tilde{\psi}(q, \cdot)=0$ on $\partial \Omega$ and renormalizing it. Indeed the strong maximum principle ensures that $\frac{\partial \tilde{\psi}}{\partial n}(q, \cdot)<0$ on $\partial \mathcal{S}(q)$, so that $\int_{\partial \mathcal{S}(q)} \frac{\partial \tilde{\psi}}{\partial n}(q, \cdot) \mathrm{d} s<0$. 
The function $C(q)$ is actually minus the inverse of the condenser capacity of $\mathcal{S}(q)$ in $\Omega$, that is, of $\int_{\mathcal{F}(q)}|\nabla \tilde{\psi}(q, \cdot)|^{2} \mathrm{~d} x$. Observe that

$$
\begin{gathered}
\forall q \in \mathcal{Q}, \quad C(q)=-\int_{\mathcal{F}(q)}|\nabla \psi(q, \cdot)|^{2} \mathrm{~d} x<0, \\
C \in C^{\infty}(\mathcal{Q} ;(-\infty, 0)) \text { and depends on } \mathcal{S}_{0} \text { and } \Omega .
\end{gathered}
$$

Concerning (2.13) and other similar properties below about the regularity with respect to the shape, we refer to $[6,17,32,40]$.

Decomposition of the fluid velocity. The fluid velocity $u$ satisfies a div-curl type system in the doubly-connected domain $\mathcal{F}(q)$, constituted of 2.1b), 2.1e, (2.1f), 2.2 and of 2.4). When the solid position $q$ in $\mathcal{Q}$, and the right hand sides of these equations, including the solid velocity $q^{\prime}=\left(q_{1}^{\prime}, q_{2}^{\prime}, q_{3}^{\prime}\right) \in \mathbb{R}^{3}$ are given, the fluid velocity $u$ is determined in a unique way and we will therefore denote it by $u(q, \cdot)$. Moreover, thanks to (2.9), 2.10) and (2.11), the solution $u(q, \cdot)$ takes the form:

$$
u(q, \cdot)=u_{q^{\prime}}(q, \cdot)+u_{\gamma}(q, \cdot)
$$

with

$$
\begin{aligned}
& u_{q^{\prime}}(q, \cdot):=\nabla\left(\varphi(q, \cdot) \cdot q^{\prime}\right)=\nabla\left(\sum_{j=1}^{3} \varphi_{j}(q, \cdot) q_{j}^{\prime}\right) \\
& \text { and } \quad u_{\gamma}(q, \cdot):=\gamma \nabla^{\perp} \psi(q, \cdot) .
\end{aligned}
$$

So besides the dependence with respect to $\mathcal{S}_{0}$, to $\Omega$ and to the space variable, $u_{q^{\prime}}$ depends on $q$ and linearly on $q^{\prime}$ while $u_{\gamma}$ depends on $q$ and linearly on $\gamma$. Notice that the initial data (2.3a) for system of equations (2.1) is no longer required since it can be deduced from the given circulation $\gamma$ and the initial data of the solid through the functions $\varphi(0, \cdot)$ and $\psi(0, \cdot)$.

Inertia matrices. We can now define the mass matrices

$$
\begin{aligned}
M_{g} & :=\operatorname{diag}(\mathcal{J}, m, m) \\
M_{a}(q) & :=\int_{\partial \mathcal{S}(q)} \varphi(q, \cdot) \otimes \frac{\partial \varphi}{\partial n}(q, \cdot) \mathrm{d} s=\left(\int_{\mathcal{F}(q)} \nabla \varphi_{i} \cdot \nabla \varphi_{j} \mathrm{~d} x\right)_{1 \leqslant i, j \leqslant 3} \\
M(q) & :=M_{g}+M_{a}(q) .
\end{aligned}
$$

The matrix $M(q)$ corresponds to the sum of the genuine inertia $M_{g}$ of the body and the so-called added inertia $M_{a}(q)$, which, loosely speaking, measures how much the surrounding fluid resists the acceleration of the body motion (since the fluid undergoes an acceleration as well). Both $M_{g}$ and $M_{a}(q)$ are symmetric and positive-semidefinite, and $M_{g}$ is positive definite. 
Christoffel symbols. A bilinear symmetric mapping $\Gamma(q)$ associated with $M(q)$ can be defined as follows, for every $p=\left(p_{1}, p_{2}, p_{3}\right) \in \mathbb{R}^{3}$ :

$$
\langle\Gamma(q), p, p\rangle:=\left(\sum_{1 \leqslant i, j \leqslant 3} \Gamma_{i, j}^{k}(q) p_{i} p_{j}\right)_{1 \leqslant k \leqslant 3} \in \mathbb{R}^{3},
$$

where, for every $i, j, k \in\{1,2,3\}$, we denote by

$$
\Gamma_{i, j}^{k}(q):=\frac{1}{2}\left(\left(M_{a}\right)_{k, j}^{i}+\left(M_{a}\right)_{k, i}^{j}-\left(M_{a}\right)_{i, j}^{k}\right)(q)
$$

the Christoffel symbols of the first kind associated with the mass matrix. In this identity, the notation $\left(M_{a}\right)_{i, j}^{k}$ stands for the partial derivative of the entry of indices $(i, j)$ of the matrix $M_{a}$ with respect to $q_{k}$, that is

$$
\left(M_{a}\right)_{i, j}^{k}:=\frac{\partial\left(M_{a}\right)_{i, j}}{\partial q_{k}} .
$$

We underline that since the genuine inertia $M_{g}$ of the body is independent of the position $q$ of the solid, only the added inertia is involved in the Christoffel symbols.

Force term. Eventually, we also define the column vectors:

$$
\begin{aligned}
& B(q):=\int_{\partial \mathcal{S}(q)}\left(\frac{\partial \psi}{\partial n}\left(\frac{\partial \varphi}{\partial n} \times \frac{\partial \varphi}{\partial \tau}\right)\right)(q, \cdot) \mathrm{d} s, \\
& E(q):=-\frac{1}{2} \int_{\partial \mathcal{S}(q)}\left(\left|\frac{\partial \psi}{\partial n}\right|^{2} \frac{\partial \varphi}{\partial n}\right)(q, \cdot) \mathrm{d} s,
\end{aligned}
$$

and for $p$ in $\mathbb{R}^{3}$ the force term

$$
F(q, p):=\gamma^{2} E(q)+\gamma p \times B(q) .
$$

We recall that $\gamma$ denotes the circulation around the body.

Remark 2.1. The notations $E$ and $B$ are chosen on purpose to highlight the analogy with the Lorentz force acting on a charged particle moving under the influence of an electromagnetic field $(E, B)$. This force vanishes if $\gamma=0$.

It can be checked that

$$
\begin{aligned}
& M \in C^{\infty}\left(\mathcal{Q} ; S_{3}^{++}(\mathbb{R})\right) \text { and depends on } \mathcal{S}_{0}, m, \mathcal{J} \text { and } \Omega, \\
& F \in C^{\infty}\left(\mathcal{Q} \times \mathbb{R}^{3} ; \mathbb{R}^{3}\right) \text { and depends on } \mathcal{S}_{0}, \gamma \text { and } \Omega, \\
& \quad \text { and vanishes when } \gamma=0, \\
& \Gamma \in C^{\infty}\left(\mathcal{Q} ; \mathcal{B} \mathcal{L}\left(\mathbb{R}^{3} \times \mathbb{R}^{3} ; \mathbb{R}^{3}\right)\right) \text { and depends on } \mathcal{S}_{0} \text { and } \Omega .
\end{aligned}
$$

Above $S_{3}^{++}(\mathbb{R})$ denotes the set of real symmetric positive-definite $3 \times 3$ matrices, $\mathcal{B} \mathcal{L}\left(\mathbb{R}^{3} \times \mathbb{R}^{3} ; \mathbb{R}^{3}\right)$ denotes the space of bilinear mappings from $\mathbb{R}^{3} \times \mathbb{R}^{3}$ to $\mathbb{R}^{3}$. 
We stress that $M$ does not depend on the circulation $\gamma$ whereas $F$ does not depend on $m$ and $\mathcal{J}$ and $\Gamma$ does not depend on $m, \gamma$ and $\mathcal{J}$. In the following, when specifying these dependences is relevant, we will denote

$$
M\left[\mathcal{S}_{0}, m, \mathcal{J}, \Omega\right], \Gamma\left[\mathcal{S}_{0}, \Omega\right] \text { and } F\left[\mathcal{S}_{0}, \gamma, \Omega\right] \text { instead of } M, \Gamma \text { and } F \text {. }
$$

Now our first result is a rephrasing of System 2.1)-(2.2) as an ordinary differential equation.

Theorem 2.2. For smooth solutions, System (2.1)-(2.2) can be recast, up to the first collision, as the second order ODE

$$
M(q) q^{\prime \prime}+\left\langle\Gamma(q), q^{\prime}, q^{\prime}\right\rangle=F\left(q, q^{\prime}\right) .
$$

Let us emphasize that (2.21) only determines the body motion. The fluid velocity $u(q, \cdot)$ is then deduced by (2.14) and 2.15).

The proof of Theorem 2.2 is postponed to Section 5

Remark 2.3. Theorem 2.2 extends [33, Theorem 1.1] where the potential case, i.e. the case where $\gamma=0$, is obtained. In that case the ODE (2.21) means that the particle is moving along the geodesics associated with the Riemann metric induced on $\mathcal{Q}$ by the matrix $M(q)$.

On the other hand in [13] another reinterpretation in term of geodesics is given: classical solutions to the PDEs driving the fluid-rigid body system are the geodesics of a Riemannian manifold of infinite dimension, in the sense that they are the critical points of an action, which is the integral over time of the total kinetic energy of the system. This result was stated in the 3D case in 13. but holds in the present setting as well, even in the case where $\gamma \neq 0$ and where the fluid is rotational. Theorem 4.4 somehow establishes that for irrotational flows this geodesic structure can be projected on the degrees of freedom corresponding to the immersed rigid particle only, at the prize of an extra Lorentz-type force for $\gamma \neq 0$.

Remark 2.4. The analysis leading to Theorem 2.2 is extended in $[10$ in the case where a control acts on a part of the external boundary. Then the remote influence of the external boundary control translates into additional force terms.

According to classical ODE theory, given $\gamma$, there exists a maximal time $T>0$ and a unique maximal solution $q$ in $C^{\infty}([0, T) ; \mathcal{Q})$ to 2.21$)$ with Cauchy data $q(0)=(0,0), q^{\prime}(0)=\left(\omega_{0}, \ell_{0}\right)$.

Moreover, $T$ is the time of the first collision of the solid with the outer boundary of the fluid domain. If there is no collision, then $T=+\infty$. This follows from Corollary 2.7 below, which itself relies on an energy argument. Indeed an important feature of the system $(2.21)$ is that it is conservative. More precisely, let us define for $(q, p)$ in $\mathcal{Q} \times \mathbb{R}^{3}$,

$$
\mathcal{E}(q, p):=\frac{1}{2} M(q) p \cdot p+U(q),
$$

where the potential energy $U(q)$ is given by

$$
U(q):=-\frac{1}{2} \gamma^{2} C(q),
$$


with $C(q)$ given by (2.11). We will prove the following in Subsection 5.5 .

Lemma 2.5. The derivative $D C(q)$ of $C(q)$ with respect to $q$ satisfies:

$$
\forall q \in \mathcal{Q}, \quad \frac{1}{2} D C(q)=E(q),
$$

where $E(q)$ is defined by 2.18b.

As a corollary of Lemma 2.5 we have the following result regarding the energy conservation.

Proposition 2.6. For any $q$ in $C^{\infty}([0, T] ; \mathcal{Q})$ satisfying 2.21$)$,

$$
\frac{d}{d t} \mathcal{E}\left(q, q^{\prime}\right)=0,
$$

We will prove Proposition 2.6 in Subsection 5.6

Let us emphasize that the energy function $\mathcal{E}$ belongs to $C^{\infty}\left(\mathcal{Q} \times \mathbb{R}^{3} ; \mathbb{R}\right)$ and is the sum of two nonnegative terms. In addition to its dependence on $q$ and $p$, the energy $\mathcal{E}$ depends on $\mathcal{S}_{0}, m, \mathcal{J}, \gamma$ and $\Omega$.

If we assume that the body remains at a distance greater than $\delta>0$ from the boundary we may infer a bound for the body velocity. Indeed we have the following immediate corollary of Proposition 2.6

Corollary 2.7. Let $\mathcal{S}_{0}$ a subset of $\Omega, p_{0}$ in $\mathbb{R}^{3}$ and $(\gamma, m, \mathcal{J})$ in $\mathbb{R} \times(0,+\infty) \times$ $(0,+\infty)$. Let $\delta>0$ and $q$ in $C^{\infty}\left([0, T] ; \mathcal{Q} \times \mathbb{R}^{3}\right)$ satisfying 2.21$)$ with the Cauchy data $\left(q, q^{\prime}\right)(0)=\left(0, p_{0}\right)$ and such that $d(\mathcal{S}(q(t)), \partial \Omega)>\delta$ for $t$ in $[0, T]$. Then there exists $K>0$ depending only on $\mathcal{S}_{0}, \Omega, p_{0}, \gamma, m, \mathcal{J}$ and $\delta$ such that $\left|q^{\prime}\right|_{\mathbb{R}^{3}} \leqslant K$ on $[0, T]$.

Let us refer here to 18 and 34 for a study of the collision of a solid moving in a potential flow (that is in the case where $\gamma=0$ ) with the fixed boundary of the fluid domain.

Let us now turn our attention back to the Christoffel symbols defined in 2.17). They actually can be split into two parts: one taking into account the effect of the solid rotation and the other part encoding the effect of the exterior boundary. First, we introduce the impulses $\rho_{g}, \rho_{a}, \rho$ in $\mathbb{R}$ and $P_{g}, P_{a}$ and $P$ in $\mathbb{R}^{2}$ by the following relations

$$
\left(\begin{array}{l}
\rho_{g} \\
P_{g}
\end{array}\right):=M_{g} p, \quad\left(\begin{array}{l}
\rho_{a} \\
P_{a}
\end{array}\right):=M_{a}(q) p, \quad\left(\begin{array}{c}
\rho \\
P
\end{array}\right):=\left(\begin{array}{c}
\rho_{g}+\rho_{a} \\
P_{g}+P_{a}
\end{array}\right) .
$$

Then for every $q$ in $\mathcal{Q}$ and for every $p=(\omega, \ell)$ in $\mathbb{R}^{3}$, we let:

$$
\left\langle\Gamma^{\mathrm{rot}}(q), p, p\right\rangle:=-\left(\begin{array}{c}
0 \\
P_{a}
\end{array}\right) \times p-\omega M_{a}(q)\left(\begin{array}{c}
0 \\
\ell^{\perp}
\end{array}\right) \in \mathbb{R}^{3} .
$$

We can notice that one also has

$$
\left\langle\Gamma^{\mathrm{rot}}(q), p, p\right\rangle=-\left(\begin{array}{c}
0 \\
P
\end{array}\right) \times p-\omega M(q)\left(\begin{array}{c}
0 \\
\ell^{\perp}
\end{array}\right) \in \mathbb{R}^{3},
$$


since the extra terms cancel out.

Next, for every $j, k, l \in\{1,2,3\}$, we set

$$
\begin{aligned}
& \left(\Gamma^{\partial \Omega}\right)_{k, l}^{j}(q):= \\
& \quad \frac{1}{2} \int_{\partial \Omega}\left(\frac{\partial \varphi_{j}}{\partial \tau} \frac{\partial \varphi_{k}}{\partial \tau} K_{l}+\frac{\partial \varphi_{j}}{\partial \tau} \frac{\partial \varphi_{l}}{\partial \tau} K_{k}-\frac{\partial \varphi_{k}}{\partial \tau} \frac{\partial \varphi_{l}}{\partial \tau} K_{j}\right)(q, \cdot) \mathrm{d} s
\end{aligned}
$$

and we associate correspondingly $\Gamma^{\partial \Omega}(q)$ a symmetric bilinear mapping in $\mathcal{B L}\left(\mathbb{R}^{3} \times \mathbb{R}^{3} ; \mathbb{R}^{3}\right)$ so that for $p=\left(p_{1}, p_{2}, p_{3}\right) \in \mathbb{R}^{3}$ :

$$
\left\langle\Gamma^{\partial \Omega}(q), p, p\right\rangle:=\left(\sum_{1 \leqslant k, l \leqslant 3}\left(\Gamma^{\partial \Omega}\right)_{k, l}^{j}(q) p_{k} p_{l}\right)_{1 \leqslant j \leqslant 3} \in \mathbb{R}^{3}
$$

The Christoffel symbols satisfy the following relation.

Proposition 2.8. For every $q$ in $\mathcal{Q}$ and for every $p$ in $\mathbb{R}^{3}$,

$$
\langle\Gamma(q), p, p\rangle=\left\langle\Gamma^{\mathrm{rot}}(q), p, p\right\rangle+\left\langle\Gamma^{\partial \Omega}(q), p, p\right\rangle .
$$

The proof of Proposition 2.8 is given in Subsection 5.4. We emphasize that in 2.28 and the expressions above, unlike 2.17c , there is no derivative with respect to $q$, that is, no more shape derivative.

We will see that in the decomposition (2.28), the term $\Gamma^{\partial \Omega}$ obeys a scaling law with respect to $\varepsilon$ different from the one of $\Gamma^{\text {rot }}$ (compare $(9.4)$ and $(9.6)$ below), which makes it of lower order.

The case where $\mathcal{S}_{0}$ is a disk is peculiar, and we focus on it for the rest of Subsection 2.1. In particular several degeneracies appear in this case:

- the added mass matrix $M_{a}(q)$ degenerates (it becomes of rank 2),

- the potentials $\varphi_{2}, \varphi_{3}$ and $\psi$ depend on $q$ only through the position $h_{c}$ of the center of the disk $\mathcal{S}(q)$, and in particular so do $E_{2}, E_{3}, B_{1}$ and $\left(M_{a, i, j}\right)_{i, j=2,3}$,

- the dynamics of the solid also degenerates in the sense that it satisfies $\mathcal{J} \vartheta^{\prime \prime}=m h^{\prime \prime} \cdot\left(h_{c}-h\right)^{\perp}$.

Above and in the sequel we denote with an index (in normal font type) the coordinates of $E$ or $B$ (we will sometimes use italic type indices for other purposes, in a way that should not be ambiguous). Note that in particular if the solid is homogeneous, $h=h_{c}$ and $\vartheta^{\prime}$ is constant over time.

As a consequence, in this case where $\mathcal{S}_{0}$ is a disk, we establish a particular reduction of the dynamics. We will use a block decomposition of the matrix $M_{a}=M_{a}(q)$ :

$$
M_{a}=:\left(\begin{array}{cc}
m_{\#} & \mu^{t} \\
\mu & M_{b}
\end{array}\right)
$$


where $M_{b}$ is a symmetric $2 \times 2$ matrix. This matrix is useful in the case where $\mathcal{S}_{0}$ is a disk of center $\zeta$ (we will use this notation later in a broader context). Of course the position $h_{c}$ of the center of $\mathcal{S}(t)$ is related to $q=(h, \vartheta)$ by

$$
h_{c}=h+R(\vartheta) \zeta .
$$

It is easy to see that $M_{b}(q)$ depends on $q$ only through $h_{c}$ so that we define

$$
\tilde{M}_{b}\left(h_{c}\right):=M_{b}(q) .
$$

We associate with the matrix field $\tilde{M}_{b}\left(h_{c}\right)$ a bilinear symmetric mapping $\Gamma_{b}\left(h_{c}\right)$ defined as follows: for $p_{b} \in \mathbb{R}^{2}$,

$$
\left\langle\Gamma_{b}\left(h_{c}\right), p_{b}, p_{b}\right\rangle:=\left(\sum_{1 \leqslant i, j \leqslant 2}\left(\Gamma_{b}\right)_{i, j}^{k}\left(h_{c}\right) p_{b, i} p_{b, j}\right)_{1 \leqslant k \leqslant 2} \in \mathbb{R}^{2},
$$

where, for every $i, j, k \in\{1,2\}$, we set

$$
\left(\Gamma_{\mathrm{b}}\right)_{i, j}^{k}\left(h_{c}\right):=\frac{1}{2}\left(\left(\tilde{M}_{\mathrm{b}}\right)_{k, j}^{i}+\left(\tilde{M}_{\mathrm{b}}\right)_{k, i}^{j}-\left(\tilde{M}_{\mathrm{b}}\right)_{i, j}^{k}\right)\left(h_{c}\right) .
$$

In this identity, the notation $\left(\tilde{M}_{\mathrm{b}}\right)_{i, j}^{k}$ stands for the partial derivative of the entry of indices $(i, j)$ of the matrix $\tilde{M}_{b}$ with respect to $\left(h_{c}\right)_{k}$, that is

$$
\left(\tilde{M}_{b}\right)_{i, j}^{k}:=\frac{\partial\left(\tilde{M}_{b}\right)_{i, j}}{\partial\left(h_{c}\right)_{k}} .
$$

The field $E(q)$ also depends on $q$ only through $h_{c}$ and we define $E_{b}\left(h_{c}\right)$ in $\mathbb{R}^{2}$ by

$$
E_{b}\left(h_{c}\right):=\left(E_{2}, E_{3}\right)^{t}(q) .
$$

In the same way, $B(q)$ depends on $q$ only through $h_{c}$ and we define $\tilde{B}_{1}\left(h_{c}\right)$ in $\mathbb{R}$ by the relation

$$
\tilde{B}_{1}\left(h_{c}\right)=B_{1}(q) .
$$

With these settings, the dynamics can be described as follows.

Theorem 2.9. In the case where $\mathcal{S}_{0}$ is a disk of center $\zeta$, for smooth solutions, System (2.1)-2.2 can be recast up to the first collision, as the following differential system

$$
\begin{aligned}
m h^{\prime \prime}+\tilde{M}_{b}\left(h_{c}\right) h_{c}^{\prime \prime}+\left\langle\Gamma_{b}\left(h_{c}\right), h_{c}^{\prime}, h_{c}^{\prime}\right\rangle & =\gamma^{2} E_{b}\left(h_{c}\right)-\gamma \tilde{B}_{1}\left(h_{c}\right)\left(h_{c}^{\prime}\right)^{\perp}, \\
h_{c}-h & =R(\vartheta) \zeta, \\
\mathcal{J} \vartheta^{\prime \prime} & =\left(h_{c}-h\right)^{\perp} \cdot m h^{\prime \prime},
\end{aligned}
$$

with Cauchy data $(h, \vartheta)(0)=0,(\vartheta, h)^{\prime}(0)=\left(\omega_{0}, \ell_{0}\right)$ in $\mathbb{R} \times \mathbb{R}^{2}$. 

(2.15).

As for Theorem 2.2 the fluid velocity $u(q, \cdot)$ is then deduced by $(2.14$ and

The proof of Theorem 2.9 is given in Subsection 5.7

We have now at our disposal all the material to deal with the limit of the dynamics when the size of the solid goes to 0 . As mentioned in the introduction, we distinguish two cases:

- Case (i): the mass of the solid is fixed (and then the solid tends to a massless point particle), and

- Case (ii): the mass tends to 0 along with the size (and then the solid tends to a point-mass particle).

2.2 Case (i): Dynamics of a solid shrinking to a point-mass particle

From now on, we suppose that $0 \in \Omega$ and we scale $\mathcal{S}_{0}$ around 0 . Precisely, for every $\varepsilon$ in $(0,1]$, we define

$$
\mathcal{S}_{0, \varepsilon}:=\varepsilon \mathcal{S}_{0}
$$

and for every $q=(\vartheta, h) \in \mathbb{R}^{3}$,

$$
\mathcal{S}_{\varepsilon}(q):=R(\vartheta) \mathcal{S}_{0, \varepsilon}+h \quad \text { and } \quad \mathcal{F}_{\varepsilon}(q)=\Omega \backslash \mathcal{S}_{\varepsilon}(q) .
$$

We recall that $h(0)=0$ so that 2.36 represents a homothety centered at $h(0)$. Without loss of generality, we suppose that for any $\varepsilon \in(0,1], \mathcal{S}_{0, \varepsilon} \subset \Omega$; it suffices to consider some $\mathcal{S}_{0, \varepsilon}$ as the initial solid position $\mathcal{S}_{0}$, if necessary.

In Case (i) the solid occupying the domain $\mathcal{S}_{\varepsilon}(q)$ is assumed to have a mass and a moment of inertia of the form

$$
m_{\varepsilon}=m \quad \text { and } \quad \mathcal{J}_{\varepsilon}=\varepsilon^{2} \mathcal{J}_{1},
$$

where $m>0$ and $\mathcal{J}_{1}>0$ are fixed.

With these settings, we denote by $q_{\varepsilon}$ the solution to the ODE (2.21) associated with

$$
M_{\varepsilon}:=M\left[\mathcal{S}_{0, \varepsilon}, m_{\varepsilon}, \mathcal{J}_{\varepsilon}, \Omega\right], \Gamma_{\varepsilon}:=\Gamma\left[\mathcal{S}_{0, \varepsilon}, \Omega\right] \text { and } F_{\varepsilon}:=F\left[\mathcal{S}_{0, \varepsilon}, \gamma, \Omega\right],
$$

in place of $M, \Gamma$ and $F$, respectively, defined on the maximal time interval $\left[0, T^{\varepsilon}\right)$. Accordingly, we define $E_{\varepsilon}$ and $B_{\varepsilon}$ so that the equivalent of $(2.18 \mathrm{c}$ ) is true for all $p \in \mathbb{R}^{3}$. As before we decompose $q_{\varepsilon}$ into

$$
q_{\varepsilon}=\left(\vartheta_{\varepsilon}, h_{\varepsilon}\right) \in \mathbb{R} \times \mathbb{R}^{2} .
$$

We emphasize that the circulation $\gamma$ and the Cauchy data $\left(q_{0}, p_{0}\right)$ do not depend on $\varepsilon$. The latter are decomposed into

$$
q_{0}=(0,0) \text { and } p_{0}=\left(\omega_{0}, \ell_{0}\right) .
$$


Our first result is the convergence, in this setting, of $h_{\varepsilon}$ to the solution to a massive point vortex equation. Let us introduce this limit equation. Let $(\bar{h}, \bar{T})$ be the maximal solution to the ODE:

$$
\begin{aligned}
m \bar{h}^{\prime \prime} & =\gamma\left(\bar{h}^{\prime}-\gamma u^{\Omega}(\bar{h})\right)^{\perp} \quad \text { for } t \in[0, \bar{T}), \\
\text { with } \quad \bar{h}(0) & =0 \text { and } \bar{h}^{\prime}(0)=\ell_{0},
\end{aligned}
$$

where $u^{\Omega}$ is the Kirchhoff-Routh velocity defined as follows. Consider first $\psi_{0}^{\Omega}(h, \cdot)$, the solution to the following Dirichlet problem:

$$
\Delta \psi_{0}^{\Omega}(h, \cdot)=0 \text { in } \Omega, \quad \psi_{0}^{\Omega}(h, \cdot)=G(\cdot-h) \text { on } \partial \Omega,
$$

where

$$
G(r):=-\frac{1}{2 \pi} \ln |r|
$$

The Kirchhoff-Routh stream function $\psi^{\Omega}$ is defined as

$$
\psi^{\Omega}(x):=\frac{1}{2} \psi_{0}^{\Omega}(x, x), \quad x \in \Omega,
$$

and the Kirchhoff-Routh velocity $u^{\Omega}$ is defined by

$$
u^{\Omega}:=\nabla^{\perp} \psi^{\Omega},
$$

where $\nabla^{\perp}:=\left(-\partial_{2}, \partial_{1}\right)$.

The existence of $(\bar{h}, \bar{T})$ follows from classical ODE theory. Moreover for any $h$ in $C^{\infty}([0, T] ; \Omega)$ satisfying 2.40 the following energy conservation holds:

$$
\frac{d}{d t} \mathcal{E}_{(i)}\left(h, h^{\prime}\right)=0, \quad \text { with } \mathcal{E}_{(i)}\left(h, h^{\prime}\right):=\frac{1}{2} m h^{\prime} \cdot h^{\prime}-\gamma^{2} \psi^{\Omega}(h)
$$

Remark 2.10. Indeed 2.40 is a Hamiltonian system associated with the energy 2.45. For further Hamiltonian aspects related to System 2.40 we refer for instance to 42 .

From 2.45 and the regularity of the Kirchhoff-Routh stream function $\psi^{\Omega}$ in $\Omega$ we deduce that $\bar{T}$ is the time of the first collision of $\bar{h}$ with the outer boundary $\partial \Omega$ of the fluid domain. If there is no collision, then $\bar{T}=+\infty$.

The precise statement of our first convergence result is as follows.

Theorem 2.11. Let $\mathcal{S}_{0}$ a subset of $\Omega$ as above, $p_{0}$ in $\mathbb{R}^{3}$ and $(\gamma, m, \mathcal{J})$ in $\mathbb{R} \times(0,+\infty) \times(0,+\infty)$. Let $(\bar{h}, \bar{T})$ be the maximal solution to 2.40 . For every $\varepsilon$ in $(0,1]$, let $\left(q_{\varepsilon}, T_{\varepsilon}\right)$ be the maximal solution to 2.21 with respectively $M_{\varepsilon}=M\left[\mathcal{S}_{0, \varepsilon}, m_{\varepsilon}, \mathcal{J}_{\varepsilon}, \Omega\right], \Gamma_{\varepsilon}=\Gamma\left[\mathcal{S}_{0, \varepsilon}, \Omega\right]$ and $F_{\varepsilon}=F\left[\mathcal{S}_{0, \varepsilon}, \gamma, \Omega\right]$ in place of $M, \Gamma$ and $F$, respectively, where $m_{\varepsilon}, \mathcal{J}_{\varepsilon}$ are given by 2.38 , and with the initial data $q_{\varepsilon}(0)=0$ and $q_{\varepsilon}^{\prime}(0)=p_{0}$. Then, as $\varepsilon \rightarrow 0^{+}$, $\liminf T_{\varepsilon} \geqslant \bar{T}$, and for all $T$ in $(0, \bar{T}), h_{\varepsilon} \rightarrow \bar{h}$ in $W^{2, \infty}\left([0, T] ; \mathbb{R}^{2}\right)$ weak $-\star$ and $\varepsilon \vartheta_{\varepsilon} \longrightarrow 0$ in $W^{2, \infty}([0, T] ; \mathbb{R})$ weak- .

Theorem 2.11 is proved in Section 6 . 
2.3 Case (ii): Dynamics of a solid shrinking to a massless point particle

In this section the solid is still assumed to occupy initially the domain $\mathcal{S}_{0, \varepsilon}$ given by 2.36) (satisfying the same assumptions as above) but we assume now that it has a mass and a moment of inertia given by

$$
m_{\varepsilon}=\alpha_{\varepsilon} m_{1} \text { and } \mathcal{J}_{\varepsilon}=\alpha_{\varepsilon} \varepsilon^{2} \mathcal{J}_{1}
$$

where $\alpha_{1}=1$ and $\alpha_{\varepsilon} \rightarrow 0^{+}$as $\varepsilon \rightarrow 0^{+}$, and where $m_{1}>0$ and $\mathcal{J}_{1}>0$ are fixed. To simplify the notations we will assume that $\alpha_{\varepsilon}$ is of the form

$$
\alpha_{\varepsilon}=\varepsilon^{\alpha}
$$

with $\alpha>0$. The particular case where $\alpha=2$ corresponds to the case of a fixed solid density. Case (i) corresponded to the case where $\alpha=0$.

In this setting, we denote by $q_{\varepsilon}=\left(\vartheta_{\varepsilon}, h_{\varepsilon}\right)$ in $\mathbb{R} \times \mathbb{R}^{2}$ the solution to the ODE (2.21) associated with $M_{\varepsilon}:=M\left[\mathcal{S}_{0, \varepsilon}, m_{\varepsilon}, \mathcal{J}_{\varepsilon}, \Omega\right], \Gamma_{\varepsilon}:=\Gamma\left[\mathcal{S}_{0, \varepsilon}, \Omega\right]$ and $F_{\varepsilon}:=$ $F\left[\mathcal{S}_{0, \varepsilon}, \gamma, \Omega\right]$ in place of $M, \Gamma$ and $F$, respectively, defined on the maximal time interval $\left[0, T^{\varepsilon}\right)$. We stress that the circulation $\gamma$ and the Cauchy data are still assumed independent of $\varepsilon$. Moreover we will assume here that

$$
\gamma \neq 0
$$

Our second result is the convergence of $h_{\varepsilon}$ as $\varepsilon \rightarrow 0^{+}$to the solution to the point vortex equation:

$$
\bar{h}^{\prime}=\gamma u^{\Omega}(\bar{h}) \text { for } t>0 \text {, with } \bar{h}(0)=0 .
$$

It is well-known that 2.48 is a Hamiltonian system associated with the energy

$$
\mathcal{E}_{(i i)}(h):=\gamma^{2} \psi^{\Omega}(h) ;
$$

see for instance to $\left[27,35\right.$. In particular for any $h$ in $C^{\infty}([0, T] ; \Omega)$ satisfying 2.48,

$$
\frac{d}{d t} \mathcal{E}_{(i i)}(h)=0 \text {. }
$$

It follows from 2.49 and the fact that $\psi^{\Omega}(h) \rightarrow+\infty$ when $h$ gets close to $\partial \Omega$, see for instance [41, Eq. (1.27)], that the solution $\bar{h}$ is global in time, and in particular that there is no collision of the point vortex with the external boundary $\partial \Omega$.

Our result in this situation is the following.

Theorem 2.12. Let $\mathcal{S}_{0}$ a subset of $\Omega$ as above, different from a disk. Let $\gamma \neq 0, p_{0}$ in $\mathbb{R}^{3}$ and $\left(m_{1}, \mathcal{J}_{1}\right)$ in $(0,+\infty) \times(0,+\infty)$. Let us consider the global solution $\bar{h}$ to 2.48 and for every $\varepsilon$ in $(0,1]$, let $\left(q_{\varepsilon}, T_{\varepsilon}\right)$ the maximal solution to 2.21 with respectively $M_{\varepsilon}=M\left[\mathcal{S}_{0, \varepsilon}, m_{\varepsilon}, \mathcal{J}_{\varepsilon}, \Omega\right], \Gamma_{\varepsilon}=\Gamma\left[\mathcal{S}_{0, \varepsilon}, \Omega\right]$ and $F_{\varepsilon}=F\left[\mathcal{S}_{0, \varepsilon}, \gamma, \Omega\right]$ in place of $M, \Gamma$ and $F$, where $m_{\varepsilon}, \mathcal{J}_{\varepsilon}$ are given by (2.46), and with the initial data $q_{\varepsilon}(0)=0$ and $q_{\varepsilon}^{\prime}(0)=p_{0}$. Then, as $\varepsilon \rightarrow 0^{+}$,

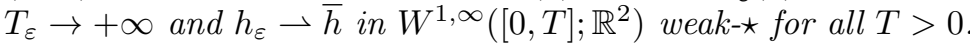


Theorem 2.12 is proved in Section 7 .

In the case where $\mathcal{S}_{0}$ is a disk, the statement needs a slight modification.

Theorem 2.13. Let $\mathcal{S}_{0}$ a disk in $\Omega$, with center $\zeta$ and center of mass 0 . Let $\gamma \neq 0,\left(m_{1}, \mathcal{J}_{1}\right)$ in $(0,+\infty)^{2}$ and $p_{0}$ in $\mathbb{R}^{3}$. Let us consider the global solution $\bar{h}$ to 2.48 and for every $\varepsilon$ in $(0,1]$, let $\left(q_{\varepsilon}, T_{\varepsilon}\right)$ be as in Theorem 2.12. Let $h_{c, \varepsilon}=h_{\varepsilon}+\varepsilon R\left(\vartheta_{\varepsilon}\right) \zeta$ the center of the disk. Then, as $\varepsilon \rightarrow 0^{+}, T_{\varepsilon} \rightarrow+\infty$ and $h_{c, \varepsilon} \rightarrow \bar{h}$ in $W^{1, \infty}\left([0, T] ; \mathbb{R}^{2}\right)$ weak-^ for all $T>0$.

Theorem 2.13 is proved in Subsection 7.5. It is straightforward to check that this involves the convergence of $h_{\varepsilon}$ to $h$ in $L^{\infty}$ strong for all $T>0$. Actually, one even gets the convergence in $W^{\beta, \infty}(0, T)$ weak- $\star$ for all $T>0$ with $\beta:=\min \left(1, \frac{2}{\alpha}\right)$; see again Subsection 7.5. As we will see during the proofs, if either $\mathcal{S}_{0}$ is homogeneous (so that the center of the disk and the center of gravity coincide) or if $\alpha \leqslant 2$, Theorem 2.12 is actually valid without change.

\section{Comments and organization of the paper}

\subsection{Comments}

The limit systems obtained in Cases (i) and (ii) do not depend on the body shape nor on the value of $\alpha>0$. Still the proof is simpler in the case where the body is a homogeneous disk. Indeed if $\mathcal{S}_{0}$ is a disk, in both Cases (i) and (ii), it follows directly from 2.1d that the rotation $\vartheta_{\varepsilon}$ satisfies, for any $\varepsilon$ in $(0,1), \vartheta_{\varepsilon}(t)=t \omega_{0}$ as long as the solution exists.

We notice that the convergences in Theorem 2.11 and Theorem 2.12 cannot be improved unless some compatibility condition holds at initial time. One may however wonder if these convergences could be improved in the open interval $(0, T)$. It seems that some strong oscillations in time show up when $\varepsilon \rightarrow 0^{+}$which prevent strong convergence from happening. We plan to study this phenomenon by a multi-scale approach of the solution to the ODE (2.21) in a forthcoming work. Once again the case where the body is a homogeneous disk is likely to simplify the discussion.

Let us also underline that the convergence of the dynamics of the solid involves some convergence in the fluid. It is not difficult to check that for any $k \in \mathbb{N}$ we have the weak- $\star$ convergence in $W^{1, \infty}\left(0, T ; C^{k}(K)\right)$ for any $T<\bar{T}$ in Case (i) and in $L^{\infty}\left(0, T ; C^{k}(K)\right)$ for any $T>0$ in Case (ii), of the fluid velocity $u_{\varepsilon}$ toward $\gamma u^{\Omega}(\cdot)+\frac{\gamma}{2 \pi} \frac{(\cdot-\bar{h}(t))^{\perp}}{|\cdot-\bar{h}(t)|^{2}}$, for any compact set $K \subset \Omega$ not intersecting $\{\bar{h}(t), t \in[0, T]\}$.

In Case (i), one may also raise the question whether it is possible that $\liminf T_{\varepsilon}>\bar{T}$. This problem should be connected to the behaviour of the potentials and stream functions as the body approaches the boundary; see for instance [3], [5], 29] and [34 and references therein for this question.

The analysis performed in this paper can be easily adapted in order to cover the case where the circulation $\gamma$ depends on $\varepsilon$ under the form $\gamma^{\varepsilon}=\varepsilon^{\beta} \gamma^{1}$ 
with $\beta>0$ in Case (i) and $\beta$ in $(0,1)$ in Case (ii). One obtains respectively at the limit the trivial equations $\bar{h}^{\prime \prime}=0$ and $\bar{h}^{\prime}=0$.

Our analysis should hold as well in the case of several bodies moving in the full plane or in a multiply-connected domain, as long as there is no collision. This will be tackled in a forthcoming work.

Another natural question is whether or not one may extend the results of Theorem 2.11 and Theorem 2.12 to rotational flows. These extensions are respectively tackled in [11] and [12] in the case without external boundary.

\subsection{Organization of the paper}

The paper is organized as follows.

In Section 4 we deal with the simpler case when there is no external boundary. This case is well-known in the literature and has been in particular studied with complex analysis, using Blasius' lemma (see e.g. [27]). We shall use an alternative approach based on a lemma due to Lamb, see Lemma 4.6, allowing one to exchange normal and tangential components in some trilinear integrals over the body boundary. The case without outer boundary is actually very important to tackle the general one.

Then Theorem 2.2, Proposition 2.6 and Proposition 2.8, which are independent of $\varepsilon$, are proved in Section 5 .

In Sections 6 and 7, respectively, we prove Theorem 2.11 concerning the limit of a massive particle (Case (i)) and Theorem 2.12 concerning the limit of a massless particle (Case (ii)). These proofs rely on some asymptotic normal forms (6.7) and (7.10), respectively. These normal forms are the key point of the demonstration and allow us to establish some renormalized and modulated energy estimates and prove the passage to the limit. They are established in the last two sections.

In Section 8 we establish some asymptotic expansions of stream and potential functions with respect to $\varepsilon$. These expansions involve two scales corresponding respectively to variations over length $O(1)$ and $O(\varepsilon)$ respectively on $\partial \Omega$ and $\partial \mathcal{S}_{\varepsilon}(q)$. The profiles appearing in these expansions are obtained by successive corrections, considering alternatively at their respective scales the body boundary from which the external boundary seems remote and the external boundary from which the body seems tiny, so that good approximations are given respectively by the case without external boundary and without the body.

Then in Section 9, we prove the normal forms (6.7) and 7.10). To do so we plug the expansions obtained in Section 8 into the expressions of the inertia matrix $M_{\varepsilon}(q)$, of the Christoffel symbol $\left\langle\Gamma_{\varepsilon}(q), p, p\right\rangle$ and of the force fields $E_{\varepsilon}$ and $p \times B_{\varepsilon}$ and compute the leading terms of the resulting expansions. These expansions can themselves be plugged into the ODE (2.21) of Theorem 2.2. In particular, thanks to Lamb's lemma we will make appear in several terms of the expansions of $E_{\varepsilon}$ and $B_{\varepsilon}$ some coefficients of the added inertia of the solid corresponding to the case without external boundary. Strikingly this allows us 
to combine the subprincipal terms of the expansions of $E_{\varepsilon}$ and $B_{\varepsilon}$ with the leading term of the expansion of $\Gamma_{\varepsilon}$ thanks to a modulation of the variable; see Lemma 9.9 . This fact is essential in the proof.

\section{Case without external boundary}

In this section, we consider a simplified version of the problem that we are interested in. The simplification consists in assuming that the domain occupied by the fluid-solid system is the whole plane, i.e. $\Omega=\mathbb{R}^{2}$, the fluid being at rest at infinity. We aim to give (in this simplified unbounded configuration) the counterparts of Theorems 2.2, 2.11 and 2.12. The rephrasing of the equations driving the dynamics as an ODE (that is a result similar to Theorem 2.2), has been known since the investigations of Blasius, Kutta, Joukowski, Chaplygin and Sedov. Their analysis, relying on a complex-analytic approach, was then revisited following another approach which seems to date back to Lamb. Since we will elaborate on the latter in order to deal with the bounded case, we will first establish the counterpart of Theorem 2.2 relying on Lamb's analysis. We will deduce from this ODE an energy conservation providing an analogous to Proposition 2.6. Then we will investigate the passage to the limit of the dynamics when the size of the solid goes to 0 in both Cases (i) and (ii). Hence we will establish the counterparts of Theorem 2.11 and Theorem 2.12

Remark 4.1. The purpose of this section is not only to provide a "warm-up" for the much more involved "bounded" configuration. It turns out that several objects that will come up in the analysis will be also of central importance in the sequel.

\subsection{Recasting of the system as an ODE}

In the case where $\Omega=\mathbb{R}^{2}$, the fluid-solid system is governed by the following set of coupled equations, quite similar to System (2.1):

$$
\begin{gathered}
\frac{\partial u}{\partial t}+(u \cdot \nabla) u+\nabla \Pi=0 \quad \text { and } \quad \operatorname{div} u=0 \text { in } \mathbb{R}^{2} \backslash \mathcal{S}(t), \\
\left(\mathcal{J} \vartheta^{\prime \prime}, m h^{\prime \prime}\right)=\left(\int_{\partial \mathcal{S}(t)}(x-h(t))^{\perp} \cdot \Pi n \mathrm{~d} s, \int_{\partial \mathcal{S}(t)} \Pi n \mathrm{~d} s\right), \\
u \cdot n=\left(\vartheta^{\prime}(\cdot-h)^{\perp}+h^{\prime}\right) \cdot n \text { on } \partial \mathcal{S}(t) \text { and } u(x) \rightarrow 0 \text { when }|x| \rightarrow+\infty .
\end{gathered}
$$

We still consider irrotational solutions:

$$
\operatorname{curl} u=0 \text { in } \mathbb{R}^{2} \backslash \mathcal{S}(t) .
$$

Again, this depends solely on the fluid part of the initial data

$$
\left.u\right|_{t=0}=u_{0} \text { in } \mathbb{R}^{2} \backslash \mathcal{S}_{0} \text { and }(\vartheta, h)(0)=(0,0),\left(\vartheta^{\prime}, h^{\prime}\right)(0)=\left(\omega_{0}, \ell_{0}\right) .
$$


To state the aforementioned reformulation, we introduce the Kirchhoff potentials, the inertia matrices, the Christoffel symbols and the force term corresponding to this simplified case.

Kirchhoff potential. Let us first denote by $\varphi_{j}^{\mathcal{S}_{0}}$, for $j=1,2,3$, the Kirchhoff potentials in $\mathbb{R}^{2} \backslash \mathcal{S}_{0}$ which are the functions that satisfy the following Neumann problem:

$$
\begin{array}{lr}
\Delta \varphi_{j}^{\mathcal{S}_{0}}=0 & \text { in } \mathbb{R}^{2} \backslash \mathcal{S}_{0}, \\
\frac{\partial \varphi_{j}^{\mathcal{S}_{0}}}{\partial n}=\left\{\begin{array}{lr}
x^{\perp} \cdot n \text { for } j=1 & \text { on } \partial \mathcal{S}_{0}, \\
e_{j-1} \cdot n, \text { for } j=2,3 & \text { at infinity. }
\end{array}\right. &
\end{array}
$$

We also define

$$
\varphi^{\mathcal{S}_{0}}:=\left(\varphi_{1}^{\mathcal{S}_{0}}, \varphi_{2}^{\mathcal{S}_{0}}, \varphi_{3}^{\mathcal{S}_{0}}\right)^{t}
$$

Stream function for the circulation term. In the same spirit as (2.11), we first introduce the function $\psi_{-1}^{\mathcal{S}_{0}}$ as the solution to

$$
\begin{aligned}
-\Delta \psi_{-1}^{\mathcal{S}_{0}} & =0 & & \text { in } \mathbb{R}^{2} \backslash \mathcal{S}_{0}, \\
\psi_{-1}^{\mathcal{S}_{0}} & =C^{\mathcal{S}_{0}} & & \text { on } \partial \mathcal{S}_{0}, \\
\psi_{-1}^{\mathcal{S}_{0}} & =O(\ln |x|) & & \text { at infinity, }
\end{aligned}
$$

where the constant $C^{\mathcal{S}_{0}}$ is such that:

$$
\int_{\partial \mathcal{S}_{0}} \frac{\partial \psi_{-1}^{\mathcal{S}_{0}}}{\partial n} \mathrm{~d} s=-1 .
$$

The existence and uniqueness of $\psi_{-1}^{\mathcal{S}_{0}}$ will be recalled below in Proposition 8.1 one can still identify $\frac{\partial \psi_{-1}^{\mathcal{S}_{0}}}{\partial n}$ as being the equilibrium measure of $\partial \mathcal{S}_{0}$.

Change of frame and decomposition of the fluid velocity. The vector field $\underline{u}$ defined from the fluid velocity $u$ by

$$
\underline{u}(t, x):=R(\vartheta(t))^{t} u(t, R(\vartheta(t)) x+h(t)),
$$

satisfies the following div-curl type system in the doubly-connected domain $\mathcal{F}_{0}$ :

$$
\begin{gathered}
\operatorname{div} \underline{u}=0 \text { and } \operatorname{curl} \underline{u}=0 \text { in } \mathbb{R}^{2} \backslash \mathcal{S}_{0} \\
\underline{u} \cdot n=\left(\underline{\ell}+\vartheta^{\prime} x^{\perp}\right) \cdot n \quad \text { on } \partial \mathcal{S}_{0} \\
\underline{u} \longrightarrow 0 \quad \text { at infinity, } \\
\int_{\partial \mathcal{S}_{0}} \underline{u} \cdot \tau \mathrm{d} s=\gamma
\end{gathered}
$$


where $\gamma:=\int_{\partial \mathcal{S}_{0}} u_{0} \cdot \tau \mathrm{d} s$ and $\underline{\ell}(t):=R(\vartheta(t))^{t} h^{\prime}(t)$.

When the right hand sides of these equations are given the auxiliary velocity field $\underline{u}$ is determined in a unique way and takes the form:

$$
\underline{u}=\underline{u}_{\left(\vartheta^{\prime}, \underline{\ell}\right)}+\underline{u}_{\gamma}
$$

where, for $\underline{\ell}=\left(\underline{\ell}_{1}, \underline{\ell}_{2}\right)$,

$$
\underline{u}_{\left(\vartheta^{\prime}, \underline{\ell}\right)}:=\nabla\left(\varphi_{1}^{\mathcal{S}_{0}} \vartheta^{\prime}+\varphi_{2}^{\mathcal{S}_{0}} \underline{\ell}_{1}+\varphi_{3}^{\mathcal{S}_{0}} \underline{\ell}_{2}\right) \quad \text { and } \quad \underline{u}_{\gamma}:=\gamma \nabla^{\perp} \psi_{-1}^{\mathcal{S}_{0}} .
$$

Below we define $M_{a, \vartheta}^{\mathcal{S}_{0}}, \Gamma_{\vartheta}^{\mathcal{S}_{0}}$ and $F_{\vartheta}^{\mathcal{S}_{0}}$ such that

$$
\begin{gathered}
M_{g}+M_{a, \vartheta}^{\mathcal{S}_{0}}=M\left[\mathcal{S}_{0}, m^{1}, \mathcal{J}^{1}, \mathbb{R}^{2}\right](q), \Gamma_{\vartheta}^{\mathcal{S}_{0}}=\Gamma\left[\mathcal{S}_{0}, \mathbb{R}^{2}\right](q) \\
\text { and } F_{\vartheta}^{\mathcal{S}_{0}}(p)=F\left[\mathcal{S}_{0}, \gamma, \mathbb{R}^{2}\right](q, p) .
\end{gathered}
$$

Remark 4.2. In particular, since the dependence on $q$ of $M, \Gamma$ and $F$ reduces to a dependence on the rotation $R(\vartheta)$ only in the case without external boundary, this dependence on $\vartheta$ is mentioned through an index.

Inertia matrices. We define the added mass matrix

$$
M_{a}^{\mathcal{S}_{0}}:=\int_{\partial \mathcal{S}_{0}} \varphi^{\mathcal{S}_{0}} \otimes \frac{\partial \varphi^{\mathcal{S}_{0}}}{\partial n} \mathrm{~d} s=\left(\int_{\mathbb{R}^{2} \backslash \mathcal{S}_{0}} \nabla \varphi_{i}^{\mathcal{S}_{0}} \cdot \nabla \varphi_{j}^{\mathcal{S}_{0}} \mathrm{~d} x\right)_{1 \leqslant i, j \leqslant 3} .
$$

This matrix is symmetric positive-semidefinite and depends only on $\mathcal{S}_{0}$. Actually it is positive definite if and only if $\mathcal{S}_{0}$ is not a disk. Moreover, when $\mathcal{S}_{0}$ is a disk centered at the center of mass, this matrix reads $M_{a}^{\mathcal{S}_{0}}=: \operatorname{diag}\left(0, m_{a}^{\mathcal{S}_{0}}, m_{a}^{\mathcal{S}_{0}}\right)$ with $m_{a}^{\mathcal{S}_{0}}>0$.

Then we can introduce the mass matrix $M_{a, \vartheta}^{\mathcal{S}_{0}}$ taking the rotation into account by

$$
M_{a, \vartheta}^{\mathcal{S}_{0}}:=\mathcal{R}(\vartheta) M_{a}^{\mathcal{S}_{0}} \mathcal{R}(\vartheta)^{t} .
$$

Above we used the notation

$$
\mathcal{R}(\vartheta):=\left(\begin{array}{cc}
1 & 0 \\
0 & R(\vartheta)
\end{array}\right) \in \mathrm{SO}(3) .
$$

Christoffel symbols. Let $\Gamma_{\vartheta}^{\mathcal{S}_{0}}$ the bilinear symmetric mapping defined for all $p=(\omega, \ell) \in \mathbb{R} \times \mathbb{R}^{2}$ by

$$
\left\langle\Gamma_{\vartheta}^{\mathcal{S}_{0}}, p, p\right\rangle:=-\left(\begin{array}{c}
0 \\
P_{a, \vartheta}^{\mathcal{S}_{0}}
\end{array}\right) \times p-\omega M_{a, \vartheta}^{\mathcal{S}_{0}}\left(\begin{array}{c}
0 \\
\ell^{\perp}
\end{array}\right),
$$

where $P_{a, \vartheta}^{\mathcal{S}_{0}}$ denotes the last two coordinates of the impulse $M_{a, \vartheta}^{\mathcal{S}_{0}} p$. We associate with $\Gamma_{\vartheta}^{\mathcal{S}_{0}}$ the coefficients $\left(\Gamma_{\vartheta}^{\mathcal{S}_{0}}\right)_{i, j}^{k}$ such that for any $\vartheta$ in $\mathbb{R}$, for any $p$ in $\mathbb{R}^{3}$,

$$
\left\langle\Gamma_{\vartheta}^{\mathcal{S}_{0}}, p, p\right\rangle:=\left(\sum_{1 \leqslant i, j \leqslant 3}\left(\Gamma_{\vartheta}^{\mathcal{S}_{0}}\right)_{i, j}^{k} p_{i} p_{j}\right)_{1 \leqslant k \leqslant 3} .
$$


Then a tedious computation reveals that for every $i, j, k \in\{1,2,3\}$,

$$
\left(\Gamma_{\vartheta}^{\mathcal{S}_{0}}\right)_{i, j}^{k}=\frac{1}{2}\left(\left(M_{a, \vartheta}^{\mathcal{S}_{0}}\right)_{k, j}^{i}+\left(M_{a, \vartheta}^{\mathcal{S}_{0}}\right)_{k, i}^{j}-\left(M_{a, \vartheta}^{\mathcal{S}_{0}}\right)_{i, j}^{k}\right)
$$

where $\left(M_{a, \vartheta}^{\mathcal{S}_{0}}\right)_{i, j}^{k}$ denotes the partial derivative with respect to $q_{k}$ of the entry of indexes $(i, j)$ of the matrix $M_{a, \vartheta}^{\mathcal{S}_{0}}$. Thus the coefficients $\left(\Gamma_{\vartheta}^{\mathcal{S}_{0}}\right)_{i, j}^{k}$, for $i, j, k \in$ $\{1,2,3\}$, are the Christoffel symbols of the first kind associated with the matrix $M_{a, \vartheta}^{\mathcal{S}_{0}}$. There is naturally no counterpart of the term $\Gamma^{\partial \Omega}$ here.

Force term. Let us define the geometric constant

$$
\zeta:=-\int_{\partial \mathcal{S}_{0}} x \frac{\partial \psi_{-1}^{\mathcal{S}_{0}}}{\partial n} \mathrm{~d} s
$$

which is a vector of $\mathbb{R}^{2}$, depending only on $\mathcal{S}_{0}$, usually referred to as the conformal center of $\mathcal{S}_{0}$. Remark that if $\mathcal{S}_{0}$ is a disk, $\psi_{-1}^{\mathcal{S}_{0}}(x)=\frac{1}{2 \pi} \ln \left|x-h_{c}(0)\right|$ where $h_{c}(0)$ is the center of the disk (recalling that the initial position of the center of mass is $h(0)=0)$; it follows that $\zeta=h_{c}(0)$ and the definition of $\zeta$ in 4.13 is coherent with the notation in Theorem 2.9.

Next we define the force term, for all $p=(\omega, \ell) \in \mathbb{R}^{3}$, as being:

$$
F_{\vartheta}^{\mathcal{S}_{0}}(p):=\gamma\left(\begin{array}{c}
\zeta_{\vartheta} \cdot \ell \\
\ell^{\perp}-\omega \zeta_{\vartheta}
\end{array}\right),
$$

where

$$
\zeta_{\vartheta}:=R(\vartheta) \zeta .
$$

An important feature of the force vector field $(\vartheta, p) \mapsto F_{\vartheta}^{\mathcal{S}_{0}}(p)$ is that it is gyroscopic, in the sense of the following definition; see for instance [1, p. 428]. Note that we use here again the convention of Remark 4.2 to put $\vartheta$ as an index.

Definition 4.3. We say that a vector field $F$ in $C^{\infty}\left(\mathbb{R} \times \mathbb{R}^{3} ; \mathbb{R}^{3}\right)$ is gyroscopic if for any $(\vartheta, p)$ in $\mathbb{R} \times \mathbb{R}^{3}, p \cdot F(\vartheta, p)=0$.

Indeed, for any $(\vartheta, p)$ in $\mathbb{R} \times \mathbb{R}^{3}$, the force $F_{\vartheta}^{\mathcal{S}_{0}}(p)$ can be written as

$$
F_{\vartheta}^{\mathcal{S}_{0}}(p)=\gamma p \times B_{\vartheta}^{\mathcal{S}_{0}} \text { with } B_{\vartheta}^{\mathcal{S}_{0}}:=\left(\begin{array}{c}
-1 \\
\zeta_{\vartheta}^{\perp}
\end{array}\right) .
$$

The next result is a reformulation of System (4.1) as an ordinary differential equation and the counterpart of Theorem 2.2 in the case without external boundary.

Theorem 4.4. For smooth solutions, System 4.1-4.2) can be recast, up to the first collision, as the second order ODE

$$
\left(M_{g}+M_{a, \vartheta}^{\mathcal{S}_{0}}\right) q^{\prime \prime}+\left\langle\Gamma_{\vartheta}^{\mathcal{S}_{0}}, q^{\prime}, q^{\prime}\right\rangle=F_{\vartheta}^{\mathcal{S}_{0}}\left(q^{\prime}\right) .
$$


Let us underline that it is understood that we associate with the body equation given by $(4.17)$ the fluid velocity $u$ such that the vector field $\underline{u}$ given by 4.6 satisfies 4.8.

The proof of Theorem 4.4 is postponed to Subsection 4.3

In addition we have the following energy conservation property.

Proposition 4.5. If $q$ is a smooth solution to 4.17) then during its lifetime the quantity $\left(M_{g}+M_{a, \vartheta}^{\mathcal{S}_{0}}\right) q^{\prime} \cdot q^{\prime}$ is conserved.

The quantity above corresponds to twice the sum of the kinetic energy of the solid associated with the genuine inertia and of the one associated with the added inertia.

Proof For any $\vartheta$ in $\mathbb{R}$, for any $p$ in $\mathbb{R}^{3}$, we define the matrix

$$
S_{\vartheta}^{\mathcal{S}_{0}}(p):=\left(\sum_{1 \leqslant i \leqslant 3}\left(\Gamma_{\vartheta}^{\mathcal{S}_{0}}\right)_{i, j}^{k} p_{i}\right)_{1 \leqslant k, j \leqslant 3} \text { so that }\left\langle\Gamma_{\vartheta}^{\mathcal{S}_{0}}, p, p\right\rangle=S_{\vartheta}^{\mathcal{S}_{0}}(p) p
$$

Then, an explicit computation (another method, more theoretical, is given in the proof of Proposition 2.6 see Lemma 5.12 proves that for any $\vartheta$ in $\mathbb{R}$, for any $p$ in $\mathbb{R}^{3}$,

$$
\frac{1}{2} \frac{\partial M_{a, \vartheta}^{\mathcal{S}_{0}}}{\partial q}(\vartheta) \cdot p-S_{\vartheta}^{\mathcal{S}_{0}}(p) \text { is skew-symmetric. }
$$

Then Proposition 4.5 follows from 4.18 and from the fact that the force $F_{\vartheta}^{\mathcal{S}_{0}}(p)$ is gyroscopic in the sense of Definition 4.3

As a consequence, given $\gamma$ and $\mathcal{S}_{0}$ and some Cauchy data, there exists a unique global smooth solution to 4.17).

\subsection{Lamb's lemma}

To prove Theorem 4.4, we start with recalling a technical result borrowed from 23. Article 134a. (3) and (7)] and which is a cornerstone of our analysis. We recall that $\xi_{j}$ and $K_{j}$, for $j=1,2,3$, were defined in (2.7) and 2.8) respectively.

Lemma 4.6. For any pair of vector fields $u$, $v$ in $C^{\infty}\left(\overline{\mathbb{R}^{2} \backslash \mathcal{S}_{0}} ; \mathbb{R}^{2}\right)$ satisfying

$-\operatorname{div} u=\operatorname{div} v=\operatorname{curl} u=\operatorname{curl} v=0$,

$-u(x)=O(1 /|x|)$ and $v(x)=O(1 /|x|)$ as $|x| \rightarrow+\infty$,

for any $j=1,2,3$,

$$
\int_{\partial \mathcal{S}_{0}}(u \cdot v) K_{j}(0, \cdot) \mathrm{d} s=\int_{\partial \mathcal{S}_{0}} \xi_{j}(0, \cdot) \cdot((u \cdot n) v+(v \cdot n) u) \mathrm{d} s .
$$


Proof Let us start with the case where $j=2$ or 3 . Then

$$
\int_{\partial \mathcal{S}_{0}}(u \cdot v) K_{j}(0, \cdot) \mathrm{d} s=\int_{\mathbb{R}^{2} \backslash \mathcal{S}_{0}} \operatorname{div}\left((u \cdot v) \xi_{j}(0, \cdot)\right) \mathrm{d} x,
$$

since $u(x)=O(1 /|x|)$ and $v(x)=O(1 /|x|)$ when $|x| \rightarrow+\infty$. Therefore

$$
\begin{aligned}
\int_{\partial \mathcal{S}_{0}}(u \cdot v) K_{j}(0, \cdot) \mathrm{d} s & =\int_{\mathbb{R}^{2} \backslash \mathcal{S}_{0}} \xi_{j}(0, \cdot) \cdot \nabla(u \cdot v) \mathrm{d} x \\
& =\int_{\mathbb{R}^{2} \backslash \mathcal{S}_{0}} \xi_{j}(0, \cdot) \cdot(u \cdot \nabla v+v \cdot \nabla u) \mathrm{d} x,
\end{aligned}
$$

since $\operatorname{curl} u=\operatorname{curl} v=0$. Now, integrating by parts, using that $\operatorname{div} u=\operatorname{div} v=$ 0 and once again that $u(x)=O(1 /|x|)$ and $v(x)=O(1 /|x|)$ as $|x| \rightarrow+\infty$, we obtain 4.19 when $j=2$ or 3 .

We now tackle the case where $j=1$. We follow the same lines as above, with two precisions. First we observe that there is no contribution at infinity in 4.20 and 4.21) when $j=1$ as well. Indeed $\xi_{1}$ and the normal to a centered circle are orthogonal. Moreover there is no additional distributed term coming from the integration by parts in 4.21 when $j=1$ since

$\int_{\mathbb{R}^{2} \backslash \mathcal{S}_{0}} v \cdot\left(u \cdot \nabla_{x} \xi_{j}(0, \cdot)\right)+u \cdot\left(v \cdot \nabla_{x} \xi_{j}(0, \cdot)\right) \mathrm{d} x=\int_{\mathbb{R}^{2} \backslash \mathcal{S}_{0}}\left(v \cdot u^{\perp}+u \cdot v^{\perp}\right) \mathrm{d} x=0$.

The proof is then complete.

\subsection{Proof of Theorem 4.4}

To transfer the equations in the body frame we recast the equations in terms of the vector field $\underline{u}$ (defined from the fluid velocity $u$ by 4.6 ), of $\underline{\ell}(t)=$ $R(\vartheta(t))^{t} h^{\prime}(t)$ and of the auxiliary pressure $\underline{\Pi}$, defined from the fluid pressure $\Pi$ by $\underline{\Pi}(t, x)=\Pi(t, R(\vartheta(t)) x+h(t))$. Equations (4.1) become

$$
\begin{gathered}
\frac{\partial \underline{u}}{\partial t}+\left[\left(\underline{u}-\underline{\ell}-\vartheta^{\prime} x^{\perp}\right) \cdot \nabla\right] \underline{u}+\vartheta^{\prime} \underline{u}^{\perp}+\nabla \underline{\Pi}=0 \text { for } x \in \mathbb{R}^{2} \backslash \mathcal{S}_{0} \\
\qquad \operatorname{div} \underline{u}=0 \text { for } x \in \mathbb{R}^{2} \backslash \mathcal{S}_{0}, \\
\underline{u} \cdot n=\left(\underline{\ell}+\vartheta^{\prime} x^{\perp}\right) \cdot n \text { for } x \in \partial \mathcal{S}_{0} \text { and } \underline{u}(x) \rightarrow 0 \text { as }|x| \rightarrow+\infty, \\
\left(\mathcal{J} \vartheta^{\prime \prime}, m \underline{\ell}^{\prime}\right)=\left(\int_{\partial \mathcal{S}_{0}} x^{\perp} \cdot \underline{\Pi} n d s, \int_{\partial \mathcal{S}_{0}} \underline{\Pi} n d s-m \vartheta^{\prime} \underline{\ell}^{\perp}\right) .
\end{gathered}
$$

Let us consider the right hand side of 4.22d). Using an integration by parts (which we can justify by the decay at infinity of the solution, see [11, p. 520]), we obtain

$$
\left(\int_{\partial \mathcal{S}_{0}} \underline{\Pi} x^{\perp} \cdot n d s, \int_{\partial \mathcal{S}_{0}} \underline{\Pi} n \mathrm{~d} s\right)=\left(\int_{\mathbb{R}^{2} \backslash \mathcal{S}_{0}} \nabla \underline{\Pi} \cdot \nabla \varphi_{i}^{\mathcal{S}_{0}} d x\right)_{i=1,2,3} .
$$


Moreover since the vector field $\underline{u}$ is irrotational we infer from the equation 4.22a that

$$
\nabla \underline{\Pi}=-\frac{\partial \underline{u}}{\partial t}-\nabla Q, \text { with } Q:=\frac{1}{2}|\underline{u}|^{2}-\left(\underline{\ell}+\vartheta^{\prime} x^{\perp}\right) \cdot \underline{u} .
$$

Thus the solid equations $4.22 \mathrm{~d}$ ) now read

$$
\begin{aligned}
M_{g} \underline{p}^{\prime} & +m \vartheta^{\prime}\left(\begin{array}{c}
0 \\
\ell^{\perp}
\end{array}\right) \\
& =-\left(\int_{\mathbb{R}^{2} \backslash \mathcal{S}_{0}} \frac{\partial \underline{u}}{\partial t} \cdot \nabla \varphi_{i}^{\mathcal{S}_{0}} d x\right)_{i=1,2,3}-\left(\int_{\mathbb{R}^{2} \backslash \mathcal{S}_{0}} \nabla Q \cdot \nabla \varphi_{i}^{\mathcal{S}_{0}} d x\right)_{i=1,2,3}
\end{aligned}
$$

Integrating by parts, taking into account the boundary condition $4.22 \mathrm{c}$, and recalling that the added mass matrix $M_{a}^{\mathcal{S}_{0}}$ is defined in 4.9 we observe that the contribution of $\frac{\partial \underline{u}}{\partial t}$ is

$$
\left(\int_{\mathbb{R}^{2} \backslash \mathcal{S}_{0}} \frac{\partial \underline{u}}{\partial t} \cdot \nabla \varphi_{i}^{\mathcal{S}_{0}} d x\right)_{i=1,2,3}=M_{a}^{\mathcal{S}_{0}} \underline{p}^{\prime}, \text { where } \underline{p}:=\left(\begin{array}{c}
\vartheta^{\prime} \\
\underline{\ell}
\end{array}\right) .
$$

By another integration by parts we also have, for $i=1,2,3$, that

$$
\int_{\mathbb{R}^{2} \backslash \mathcal{S}_{0}} \nabla Q \cdot \nabla \varphi_{i}^{\mathcal{S}_{0}} d x=\frac{1}{2} \int_{\partial \mathcal{S}_{0}}|\underline{u}|^{2} K_{i} \mathrm{~d} s-\int_{\partial \mathcal{S}_{0}}\left(\underline{\ell}+\vartheta^{\prime} x^{\perp}\right) \cdot \underline{u} K_{i} \mathrm{~d} s
$$

where we simplified the notation by writing $K_{i}$ instead of $K_{i}(0, \cdot)$ (defined in (2.8), which corresponds to the initial position of the solid, that is $q=0$. In the same way, we will write $\xi_{i}$ for the vector fields $\xi_{i}(0, \cdot)$ (defined in (2.7)) in the computations below. Thus the solid equations $4.22 \mathrm{~d}$ can be rewritten in the form

$$
\begin{aligned}
\left(M_{g}+M_{a}^{\mathcal{S}_{0}}\right) \underline{p}^{\prime} & +m \vartheta^{\prime}\left(\begin{array}{c}
0 \\
\ell^{\perp}
\end{array}\right) \\
& =-\left(\frac{1}{2} \int_{\partial \mathcal{S}_{0}}|\underline{u}|^{2} K_{i} \mathrm{~d} s-\int_{\partial \mathcal{S}_{0}}\left(\underline{\ell}+\vartheta^{\prime} x^{\perp}\right) \cdot \underline{u} K_{i} \mathrm{~d} s\right)_{i=1,2,3}
\end{aligned}
$$

We compute the first term in the right hand side by using Lamb's lemma and the boundary conditions. For $i=1,2,3$,

$$
\begin{aligned}
\frac{1}{2} \int_{\partial \mathcal{S}_{0}}|\underline{u}|^{2} K_{i} \mathrm{~d} s=\int_{\partial \mathcal{S}_{0}}\left(\left(\underline{\ell}+\vartheta^{\prime} x^{\perp}\right) \cdot n\right)(\underline{u} \cdot n) K_{i} \mathrm{~d} s \\
\quad+\int_{\partial \mathcal{S}_{0}}\left(\left(\underline{\ell}+\vartheta^{\prime} x^{\perp}\right) \cdot n\right)(\underline{u} \cdot \tau)\left(\xi_{i} \cdot \tau\right) \mathrm{d} s .
\end{aligned}
$$


It follows that the solid equations $4.22 \mathrm{~d}$ can be recast as follows:

$$
\begin{aligned}
&\left(M_{g}+M_{a}^{\mathcal{S}_{0}}\right) \underline{p}^{\prime}+m \vartheta^{\prime}\left(\begin{array}{c}
0 \\
\underline{\ell}^{\perp}
\end{array}\right) \\
&=-\left(\sum_{k=1}^{3} \underline{p}_{k} \int_{\partial \mathcal{S}_{0}}(\underline{u} \cdot \tau)\left[\left(\xi_{i} \cdot \tau\right) K_{k}-\left(\xi_{k} \cdot \tau\right) K_{i}\right] \mathrm{d} s\right)_{i=1,2,3}
\end{aligned}
$$

We observe that the brackets above are either vanishing (for $i=k$ ) or given by the following identities:

$$
\begin{gathered}
\left(\xi_{2} \cdot \tau\right) K_{3}-\left(\xi_{3} \cdot \tau\right) K_{2}=1, \quad\left(\xi_{2} \cdot \tau\right) K_{1}-\left(\xi_{1} \cdot \tau\right) K_{2}=x^{\perp} \cdot e_{2}, \\
\text { and }\left(\xi_{3} \cdot \tau\right) K_{1}-\left(\xi_{1} \cdot \tau\right) K_{3}=-x^{\perp} \cdot e_{1} .
\end{gathered}
$$

Thanks to these equalities we compute the previous integrals in terms of the entries of the matrix $M_{a}^{\mathcal{S}_{0}}$. Precisely we decompose $M_{a}^{\mathcal{S}_{0}}$ into

$$
M_{a}^{\mathcal{S}_{0}}=:\left(\begin{array}{cc}
m_{\#}^{\mathcal{S}_{0}} & \left(\mu^{\mathcal{S}_{0}}\right)^{t} \\
\mu^{\mathcal{S}_{0}} & M_{b}^{\mathcal{S}_{0}}
\end{array}\right)
$$

where $M_{b}^{\mathcal{S}_{0}}$ is a symmetric $2 \times 2$ matrix. Then we have the following result.

Lemma 4.7. There holds:

$$
\int_{\partial \mathcal{S}_{0}}(\underline{u} \cdot \tau) x^{\perp} \mathrm{d} s=\gamma \zeta^{\perp}-\vartheta^{\prime} \mu-M_{b}^{\mathcal{S}_{0}} \underline{\ell} .
$$

Proof We first use 4.8 and 4.13 to get

$$
\int_{\partial \mathcal{S}_{0}}(\underline{u} \cdot \tau) x^{\perp} \mathrm{d} s=\gamma \zeta^{\perp}+\sum_{j=1}^{3} \underline{p}_{j} \int_{\partial \mathcal{S}_{0}}\left(\nabla \varphi_{j}^{\mathcal{S}_{0}} \cdot \tau\right) x^{\perp} \mathrm{d} s .
$$

Therefore, it only remains to observe that an integration by parts yields:

$$
\int_{\partial \mathcal{S}_{0}}\left(\nabla \varphi_{j}^{\mathcal{S}_{0}} \cdot \tau\right) x^{\perp} \mathrm{d} s=-\int_{\partial \mathcal{S}_{0}} \varphi_{j}^{\mathcal{S}_{0}} n \mathrm{~d} s=-\left(\left(M_{a}^{\mathcal{S}_{0}}\right)_{i, j}\right)_{i=2,3} .
$$

Combining now $4.7 \mathrm{~d}, 4.23$, 4.24 and 4.26 we end up with the identity:

$$
\left[M_{g}+M_{a}^{\mathcal{S}_{0}}\right] \underline{p}^{\prime}+m \vartheta^{\prime}\left(\begin{array}{c}
0 \\
\underline{\ell}^{\perp}
\end{array}\right)+\left(\begin{array}{c}
\underline{\ell}^{\perp} \cdot M_{b}^{\mathcal{S}_{0}} \underline{\ell} \\
\vartheta^{\prime}\left(M_{b}^{\mathcal{S}_{0}} \underline{\ell}\right)^{\perp}
\end{array}\right)+\vartheta^{\prime} \underline{p} \times\left(\begin{array}{c}
0 \\
\mu
\end{array}\right)=\gamma \underline{p} \times\left(\begin{array}{c}
-1 \\
\zeta^{\perp}
\end{array}\right) .
$$

Going back to the original frame we arrive at 4.17 ). This concludes the proof of Theorem 4.4.

Now in the remaining subsections we will make use of the reformulation of the system established in Theorem 4.4 to investigate the passage to the limit of the dynamics when the size of the solid goes to $0^{+}$in both Cases (i) and (ii). We will establish the counterparts of Theorem 2.11 and Theorem 2.12. 
4.4 Scaling with respect to $\varepsilon$

In this subsection we investigate the scaling of several objects with respect to $\varepsilon$ in the absence of outer boundary. We will treat at once both Cases (i) and (ii). Recall that the scaling of the inertia is given by the relations 2.46) and (2.47), that is to say, Case (i) corresponds to $\alpha=0$ in (2.47) while Case (ii) occurs when $\alpha>0$. We consider for $\varepsilon$ in $(0,1]$, the scaled solid occupying initially the domain $\mathcal{S}_{0, \varepsilon}$ given by $(2.36)$. We recall that $\gamma$ and the Cauchy data $p_{0}=\left(\omega_{0}, \ell_{0}\right)$ are supposed to be independent of $\varepsilon$ whereas $q_{0}=0$. We denote by $q_{\varepsilon}:=\left(\vartheta_{\varepsilon}, h_{\varepsilon}\right)$ in $\mathbb{R} \times \mathbb{R}^{2}$ the solution to the ODE obtained from 4.17) by rescaling the coefficients following the relations (2.36), 2.46) and (2.47).

Genuine inertia matrix and kinetic energy. Under the relation (2.46) and (2.47), the matrix of genuine inertia reads

$$
M_{g, \varepsilon}=\varepsilon^{\alpha} I_{\varepsilon} M_{g} I_{\varepsilon}
$$

where we define

$$
I_{\varepsilon}:=\operatorname{diag}(\varepsilon, 1,1) \text { and } M_{g}:=\operatorname{diag}\left(\mathcal{J}_{1}, m_{1}, m_{1}\right) .
$$

Therefore, it is natural to introduce the vector

$$
p_{\varepsilon}:=I_{\varepsilon} q_{\varepsilon}^{\prime}=\left(\begin{array}{c}
\varepsilon \vartheta_{\varepsilon}^{\prime} \\
h_{\varepsilon}^{\prime}
\end{array}\right)
$$

In particular the solid kinetic energy of the solid can be recast as

$$
\frac{1}{2} M_{g, \varepsilon} q_{\varepsilon}^{\prime} \cdot q_{\varepsilon}^{\prime}=\frac{1}{2} \varepsilon^{\alpha} M_{g} p_{\varepsilon} \cdot p_{\varepsilon}
$$

Hence the natural counterpart to $h_{\varepsilon}^{\prime}$ for what concerns the angular velocity is rather $\varepsilon \vartheta_{\varepsilon}^{\prime}$ than $\vartheta_{\varepsilon}^{\prime}$. This can also be seen on the boundary condition (2.1e): when $x$ belongs to $\partial \mathcal{S}_{\varepsilon}(t)$, the term $\vartheta_{\varepsilon}^{\prime}\left(x-h_{\varepsilon}\right)^{\perp}$ is of order $\varepsilon \vartheta_{\varepsilon}^{\prime}$ and is added to $h_{\varepsilon}^{\prime}$.

Added inertia matrix. We recall that $M_{a}^{\mathcal{S}_{0}}$ is the added inertia matrix for $\mathcal{S}_{0}$ defined in 4.9 , while $M_{a, \vartheta}^{\mathcal{S}_{0}}$ is the one corresponding to $\mathcal{S}(q)=R(\vartheta) \mathcal{S}_{0}+h$ (with $q=(\vartheta, h)$ ), given in (4.10). The scaled version of these matrices are $M_{a, \varepsilon}^{\mathcal{S}_{0}}$ corresponding to the solid $\mathcal{S}_{0, \varepsilon}$ and $M_{a, \vartheta, \varepsilon}^{\mathcal{S}_{0}}$ corresponding to $\mathcal{S}_{\varepsilon}(q)$. Then one easily sees after suitable scaling/rotation arguments that

$$
M_{a, \varepsilon}^{\mathcal{S}_{0}}=\varepsilon^{2} I_{\varepsilon} M_{a}^{\mathcal{S}_{0}} I_{\varepsilon} \text { and } M_{a, \vartheta, \varepsilon}^{\mathcal{S}_{0}}=\varepsilon^{2} I_{\varepsilon} M_{a, \vartheta}^{\mathcal{S}_{0}} I_{\varepsilon}
$$

So the dependence of the added inertia matrices with respect to $\varepsilon$ is quite simple. It will not be the case any longer in the case $\Omega$ bounded, where this dependence will be much more intricate; see Proposition 9.1 below.

Other terms and scaled equation. The other terms in (4.17) have also a simple scaling with respect to $\varepsilon$ in the unbounded case. Concerning the Christoffel 
symbols 4.12, it is not hard to check that $\left\langle\Gamma_{\vartheta, \varepsilon}^{\mathcal{S}_{0}}, q_{\varepsilon}^{\prime}, q_{\varepsilon}^{\prime}\right\rangle=\varepsilon I_{\varepsilon}\left\langle\Gamma_{\vartheta}^{\mathcal{S}_{0}}, p_{\varepsilon}, p_{\varepsilon}\right\rangle$, and concerning the force term 4.14 that $F_{\vartheta, \varepsilon}^{\mathcal{S}_{0}}\left(q_{\varepsilon}^{\prime}\right)=I_{\varepsilon} F_{\vartheta}^{\mathcal{S}_{0}}\left(p_{\varepsilon}\right)$. The counterpart of Equation 4.17) for a shrinking solid is therefore

$$
\left(\varepsilon^{\alpha} M_{g}+\varepsilon^{2} M_{a, \vartheta_{\varepsilon}}^{\mathcal{S}_{0}}\right) p_{\varepsilon}^{\prime}+\varepsilon\left\langle\Gamma_{\vartheta_{\varepsilon}}^{\mathcal{S}_{0}}, p_{\varepsilon}, p_{\varepsilon}\right\rangle=F_{\vartheta_{\varepsilon}}^{\mathcal{S}_{0}}\left(p_{\varepsilon}\right) .
$$

As we can see here, a difficulty is that the term $F_{\vartheta_{\varepsilon}}^{\mathcal{S}_{0}}$ depends on the unknown $\varepsilon \vartheta_{\varepsilon}$ through $\vartheta_{\varepsilon}$, that is singularly. This difficulty, which is still present in the general case, will be overcome by using some averaging effect; see 4.38 and Lemma 4.10 below.

Rescaled energy. Let, for any $\vartheta$ in $\mathbb{R}$ and for any $\varepsilon$ in $(0,1)$,

$$
M_{\vartheta}(\varepsilon):= \begin{cases}M_{g}+\varepsilon^{2-\alpha} M_{a, \vartheta}^{\mathcal{S}_{0}} & \text { if } \alpha \leqslant 2, \\ M_{a, \vartheta}^{\mathcal{S}_{0}}+\varepsilon^{\alpha-2} M_{g} & \text { if } \alpha>2 .\end{cases}
$$

Observe that

$$
\varepsilon^{\alpha} M_{g}+\varepsilon^{2} M_{a, \vartheta}^{\mathcal{S}_{0}}=\varepsilon^{\min (2, \alpha)} M_{\vartheta}(\varepsilon),
$$

and that this matrix is of order $O(1)$ with respect to $\varepsilon$. Using 4.29, 4.30 and 4.31 we obtain that the total energy is $\varepsilon^{\min (2, \alpha)} \mathcal{E}_{\vartheta}\left(\varepsilon, p_{\varepsilon}\right)$, where for any $p$ in $\mathbb{R}^{3}$,

$$
\mathcal{E}_{\vartheta}(\varepsilon, p):=\frac{1}{2} M_{\vartheta}(\varepsilon) p \cdot p
$$

The use of $\mathcal{E}_{\vartheta}(\varepsilon, p)$ is motivated by the following elementary result.

Lemma 4.8. Suppose that $\mathcal{S}_{0}$ is not a disk or $\alpha \leqslant 2$. There exists $K>0$ depending only on $\mathcal{S}_{0}, m_{1}$ and $\mathcal{J}_{1}$ such that, for any $(\varepsilon, \vartheta, p)$ in $(0,1) \times \mathbb{R} \times \mathbb{R}^{3}$,

$$
K|p|_{\mathbb{R}^{3}}^{2} \leqslant \mathcal{E}_{\vartheta}(\varepsilon, p) \leqslant K^{-1}|p|_{\mathbb{R}^{3}}^{2} .
$$

Using the energy conservation provided by Proposition 4.5 we deduce that, unless $\mathcal{S}_{0}$ is a disk and $\alpha>2,\left(\left|p_{\varepsilon}\right|\right)_{\varepsilon \in(0,1)}$ is bounded uniformly on $[0,+\infty)$ in both Cases (i) and (ii). (Obtaining such an a priori estimate when $\Omega$ is a bounded domain will be much more involved in particular in Case (ii).)

In the degenerate case when $\mathcal{S}_{0}$ is a disk and $\alpha>2$ (hence in Case (ii)), the problem is that $M_{a, \vartheta}^{\mathcal{S}_{0}}$ is both the principal part of $M_{\vartheta}(\varepsilon)$ and degenerate. However using (5.67) below, we can check that for $p=(\omega, \ell)$,

$$
M_{a, \vartheta}^{\mathcal{S}_{0}} p \cdot p=M_{b}^{\mathcal{S}_{0}}\left(\ell+\omega \zeta_{\vartheta}^{\perp}\right) \cdot\left(\ell+\omega \zeta_{\vartheta}^{\perp}\right),
$$

where $M_{b}^{\mathcal{S}_{0}}$ is given in 4.25. We obtain that

$$
\mathcal{E}_{\vartheta_{\varepsilon}}\left(\varepsilon, p_{\varepsilon}\right)=\frac{1}{2}\left(\varepsilon^{\alpha-2} m_{1}\left|h_{\varepsilon}^{\prime}\right|^{2}+\varepsilon^{\alpha} \mathcal{J}_{1}\left|\vartheta_{\varepsilon}^{\prime}\right|^{2}+M_{b}^{\mathcal{S}_{0}} h_{c, \varepsilon}^{\prime} \cdot h_{c, \varepsilon}^{\prime}\right),
$$

where we recall that $h_{c, \varepsilon}=h_{\varepsilon}+\varepsilon R\left(\vartheta_{\varepsilon}\right) \zeta$.

We deduce in this case that the families $\left(h_{c, \varepsilon}^{\prime}\right),\left(\varepsilon^{\frac{\alpha}{2}-1} h_{\varepsilon}^{\prime}\right)$ and $\left(\varepsilon^{\frac{\alpha}{2}} \vartheta_{\varepsilon}^{\prime}\right)$ for $\varepsilon$ in $(0,1)$, are bounded uniformly on $[0,+\infty)$.

Now our goal is to pass to the limit in each term of 4.32 . We distinguish Case (i) (with $m_{\varepsilon}, \mathcal{J}_{\varepsilon}$ given by (2.38)) and Case (ii) (with $m_{\varepsilon}, \mathcal{J}_{\varepsilon}$ given by (2.46) 
4.5 Case (i) without external boundary

In Case (i) we show that $h_{\varepsilon}$ converges to the solution to a massive point vortex equation similar to 2.40) with a vanishing Kirchhoff-Routh velocity. For $\gamma \neq 0$ we let for $t \geqslant 0$,

$$
\bar{h}(t):=\frac{m}{\gamma}\left[R\left(\frac{\gamma t}{m}-\frac{\pi}{2}\right) \ell_{0}+\ell_{0}^{\perp}\right],
$$

and $\bar{h}(t):=\ell_{0} t$ for $\gamma=0$. Of course $\bar{h}$ satisfies

$$
m \bar{h}^{\prime \prime}=\gamma\left(\bar{h}^{\prime}\right)^{\perp} \text { for } t \geqslant 0, \text { with } \bar{h}(0)=0 \text { and } \bar{h}^{\prime}(0)=\ell_{0} .
$$

The precise statement of our first convergence result is as follows.

Theorem 4.9. Let $\mathcal{S}_{0}$ a subset of $\mathbb{R}^{2}$ as above, $p_{0}$ in $\mathbb{R}^{3}$ and $(\gamma, m, \mathcal{J})$ in $\mathbb{R} \times(0,+\infty) \times(0,+\infty)$. Let $\bar{h}$ be the global solution to 4.36$)$. For every $\varepsilon$ in $(0,1]$, let $q_{\varepsilon}$ be the global solution to (4.29) and (4.32) where $m_{\varepsilon}$ and $\mathcal{J}_{\varepsilon}$ are given by (2.38), and with the initial data $q_{\varepsilon}(0)=0$ and $q_{\varepsilon}^{\prime}(0)=p_{0}$. Then, for

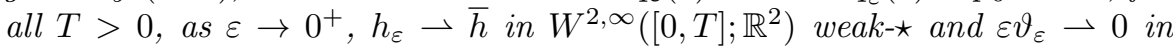
$W^{2, \infty}([0, T] ; \mathbb{R})$ weak-ᄎ.

Proof Let $T>0$. Using that $\left(p_{\varepsilon}\right)_{\varepsilon \in(0,1)}$ is bounded uniformly on $[0, T]$ and Equation 4.32, we deduce uniform $W^{2, \infty}$ bounds on $h_{\varepsilon}$ and $\varepsilon \vartheta_{\varepsilon}$. This entails the existence of a converging subsequence $\left(\varepsilon_{n} \vartheta_{\varepsilon_{n}}, h_{\varepsilon_{n}}\right)$ of $\left(\varepsilon \vartheta_{\varepsilon}, h_{\varepsilon}\right)$ :

$$
\left(\varepsilon_{n} \vartheta_{\varepsilon_{n}}, h_{\varepsilon_{n}}\right) \longrightarrow\left(\Theta_{*}, h_{*}\right) \text { in } W^{2, \infty} \text { weak- } .
$$

We now aim at characterizing the limit. First it is clear that the left hand side of 4.32 converges to $M_{g}\left(\Theta_{*}^{\prime \prime}, h_{*}^{\prime \prime}\right)^{t}$ in $L^{\infty}$ weak $-\star$. Now consider the force term $F_{\vartheta}^{\mathcal{S}_{0}}\left(p_{\varepsilon_{n}}\right)=\gamma\left(\zeta_{\vartheta_{\varepsilon_{n}}} \cdot h_{\varepsilon_{n}}^{\prime},\left(h_{\varepsilon_{n}}^{\prime}\right)^{\perp}-\varepsilon_{n} \vartheta_{\varepsilon_{n}}^{\prime} \zeta_{\vartheta_{\varepsilon_{n}}}\right)^{t}$ as defined in 4.14. On the one hand using 4.15) we see that

$$
\varepsilon_{n} \vartheta_{\varepsilon_{n}}^{\prime} \zeta_{\vartheta_{\varepsilon_{n}}} \longrightarrow 0 \text { in } W^{-1, \infty} \text { weak- } \star .
$$

On the other hand since the weak- $\star$ convergence in $W^{2, \infty}$ entails the strong $W^{1, \infty}$ one, we deduce that $h_{\varepsilon_{n}}^{\prime} \rightarrow h_{*}^{\prime}$ in $L^{\infty}$ and $\left(h_{*}(0), h_{*}^{\prime}(0)\right)=\left(0, \ell_{0}\right)$. Hence from the last two coordinates of System (4.32) we deduce $m_{1} h_{*}^{\prime \prime}=\gamma\left(h_{*}^{\prime}\right)^{\perp}$. Due to the uniqueness of the solution to Equation (4.36), this establishes that $h_{*}=\bar{h}$ and the convergence as $\varepsilon \rightarrow 0^{+}$of the whole sequence (not merely of a subsequence). This concludes the proof of Theorem 4.9 for the part concerning the position of the center of mass.

We now turn to the part concerning the angle, that is the convergence of $\varepsilon \vartheta_{\varepsilon}$. We will use the following lemma (see [1]).

Lemma 4.10. Let $\left(\omega_{n}\right)_{n \in \mathbb{N}}$ in $W^{1, \infty}(0, T ; \mathbb{R})^{\mathbb{N}},\left(\varepsilon_{n}\right)_{n \in \mathbb{N}}$ in $(0,+\infty)^{\mathbb{N}}$ with $\varepsilon_{n} \rightarrow 0^{+}$as $n \rightarrow+\infty$, such that $\varepsilon_{n} \omega_{n} \longrightarrow \bar{\rho}$ in $W^{1, \infty}(0, T ; \mathbb{R})$ weak-太 as $n \rightarrow+\infty$. Let $\left(w_{n}\right)_{n \in \mathbb{N}}$ in $L^{\infty}(0, T ; \mathbb{C})^{\mathbb{N}}$ such that $w_{n} \longrightarrow w$ in $L^{\infty}(0, T ; \mathbb{C})$ as $n \rightarrow+\infty$. Let $\vartheta_{n}:=\int_{0}^{t} \omega_{n}$. Suppose that, on $(0, T)$,

$$
\varepsilon_{n} \omega_{n}^{\prime}(t)=\operatorname{Re}\left[w_{n}(t) \exp \left(-i \vartheta_{n}(t)\right)\right] .
$$

Then $\bar{\rho}$ is constant on $[0, T]$. 
We consider the first coordinate of the system (4.32 (recall that $\alpha=0$ here). By the uniform estimate of $p_{\varepsilon}$ in $W^{1, \infty}(0, T)$, the system can be written as:

$$
M_{g} p_{\varepsilon}^{\prime}=F_{\vartheta_{\varepsilon}}^{\mathcal{S}_{0}}\left(p_{\varepsilon}\right)+R_{\varepsilon}
$$

where the remainder $R_{\varepsilon}$ is of order $\mathcal{O}(\varepsilon)$ in $L^{\infty}(0, T)$. We apply Lemma 4.10 to $\omega_{n}=\vartheta_{\varepsilon_{n}}^{\prime}$ and $w_{n}:=\frac{1}{\mathcal{J}_{1}}\left[\gamma\left(\zeta \cdot h_{\varepsilon_{n}}^{\prime}\right)-i \gamma\left(\zeta \cdot\left(h_{\varepsilon_{n}}^{\prime}\right)^{\perp}\right)+R_{\varepsilon_{n}, 1} e^{i \vartheta_{\varepsilon_{n}}}\right]$, where $R_{\varepsilon, 1}$ denotes the first coordinate of $R_{\varepsilon}$. The assumptions are satisfied thanks to (4.37). Hence $\Theta_{*}$ is constant; using the initial data, we infer that $\Theta_{*}=0$. This concludes the proof of Theorem 4.9 .

\subsection{Case (ii) without external boundary}

In this situation we show that the limit system is the point vortex system with a vanishing Kirchhoff-Routh velocity field, that is the trivial system $\bar{h}^{\prime}=0$. Our result is the following.

Theorem 4.11. Let $\mathcal{S}_{0}$ a subset of $\mathbb{R}^{2}$ as before, different from a disk (or $\alpha \leqslant 2), \gamma \neq 0, p_{0}$ in $\mathbb{R}^{3}$ and $(\gamma, m, \mathcal{J})$ in $\mathbb{R} \times(0,+\infty) \times(0,+\infty)$. For every $\varepsilon$ in $(0,1]$, let $q_{\varepsilon}$ be the global solution to 4.29 and 44.32 where $m_{\varepsilon}$ and $\mathcal{J}_{\varepsilon}$ are given by (2.46), and with the initial data $q_{\varepsilon}(0)=0$ and $q_{\varepsilon}^{\prime}(0)=p_{0}$. Then,

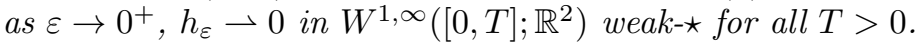

Proof First, thanks to the energy estimate, $\varepsilon M_{a, \vartheta_{\varepsilon}}^{\mathcal{S}_{0}}$ is bounded in $W^{1, \infty}$ uniformly with respect to $\varepsilon$. Since moreover $M_{g}$ is constant and $\left(p_{\varepsilon}\right)^{\prime}$ is bounded in $W^{-1, \infty}$, we can conclude that the first term in the left hand side of (4.32) converges to 0 in $W^{-1, \infty}$ (due to the extra powers of $\varepsilon$ ). Next, the second term of the left hand side converges to 0 in $L^{\infty}$ since the terms inside the brackets are bounded. Now the last two coordinates of the right hand side of the equation 4.32 correspond to the vector $\gamma\left(h_{\varepsilon}^{\prime}\right)^{\perp}-\gamma \varepsilon \vartheta_{\varepsilon}^{\prime} \zeta_{\vartheta_{\varepsilon}}$. The last term converges weakly to 0 in $W^{-1, \infty}$ as seen in Case (i); see 4.38). Hence we infer that $h_{\varepsilon}^{\prime}$ converges weakly-^ to 0 in $W^{-1, \infty}$. Because $h_{\varepsilon}^{\prime}$ is bounded, this convergences also occurs in $L^{\infty}$ weak- $\star$. This is sufficient to deduce the strong convergence of $h_{\varepsilon}$ toward some $h_{*}$ in $L^{\infty}$, and that $h_{*}^{\prime}=0$ and $h_{*}(0)=0$.

In the case of disk and for $\alpha>2$, we have the equivalent convergence for the center of the disk $h_{c, \varepsilon}$. It suffices to pass to the weak limit in $2.35 \mathrm{a}$ where, since there is no external boundary, the right hand side is simplified by $E_{\mathrm{b}}=0$ and $\tilde{B}_{1}=-1$. Note that in that case $\tilde{M}_{\mathrm{b}}$ is constant, so $\Gamma_{\mathrm{b}}=0$ (while the "original" $\Gamma$ is not, since $M_{a}$ has one more dimension and a different set of variables). When the disk is homogeneous, $h_{\varepsilon}=h_{c, \varepsilon}$, so that $h_{\varepsilon}$ converges itself in $W^{1, \infty}$ weak- $\star$. We omit the details. 
5 Recasting the system: Proofs of Theorem 2.2, Lemma 2.5 , Proposition 2.6, Proposition 2.8 and Theorem 2.9

In this Section, we prove the results of Subsection 2.1 concerning the dynamics of a solid with fixed size and mass, that is, Theorem 2.2. Lemma 2.5. Proposition 2.6. Proposition 2.8 and Theorem 2.9.

5.1 Splitting the proof of Theorem 2.2

The pressure $\Pi$ can be recovered by means of Bernoulli's formula which is obtained by combining (2.1a) and 2.2), and which reads:

$$
\nabla \Pi=-\left(\frac{\partial u}{\partial t}+\frac{1}{2} \nabla\left|u^{2}\right|\right) \quad \text { in } \mathcal{F}(q) .
$$

Given $q, p$ and $\gamma$, the pair $(u, \Pi)$ where $u$ is given by (2.14) and $\Pi$ by (5.1) yields a solution to (2.1a), (2.1b), (2.1e) and (2.1f).

Now, equations (2.1c) and (2.1d) can be summarized in the variational form:

$$
m h^{\prime \prime} \cdot \ell^{*}+\mathcal{J} \vartheta^{\prime \prime} \omega^{*}=\int_{\partial \mathcal{S}(q)} \Pi\left(\omega^{*}(x-h)^{\perp}+\ell^{*}\right) \cdot n \mathrm{~d} s,
$$

for all $p^{*}:=\left(\omega^{*}, \ell^{*}\right)$ in $\mathbb{R} \times \mathbb{R}^{2}$. Let us associate with $\left(q, p^{*}\right)$ in $\mathcal{Q} \times \mathbb{R}^{3}$ the potential vector field

$$
u^{*}:=\nabla\left(\varphi(q, \cdot) \cdot p^{*}\right),
$$

which is defined on $\mathcal{F}(q)$. By definition of the Kirchhoff potentials, see 2.9b) and $2.9 \mathrm{c})$, we have the following equalities:

$$
u^{*} \cdot n=\left(\omega^{*}(\cdot-h)^{\perp}+\ell^{*}\right) \cdot n \quad \text { on } \partial \mathcal{S}(q) \text { and } u^{*} \cdot n=0 \quad \text { on } \partial \Omega .
$$

According to Bernoulli's formula (5.1) and upon an integration by parts, identity 5.2 can be turned into:

$$
m h^{\prime \prime} \cdot \ell^{*}+\mathcal{J} \vartheta^{\prime \prime} \omega^{*}=-\int_{\mathcal{F}(q)}\left(\frac{\partial u}{\partial t}+\frac{1}{2} \nabla|u|^{2}\right) \cdot u^{*} \mathrm{~d} x,
$$

for all $p^{*}:=\left(\omega^{*}, \ell^{*}\right)$ in $\mathbb{R} \times \mathbb{R}^{2}$. Therefore substituting the decomposition 2.14 into (5.4) we arrive at

$$
\begin{aligned}
m h^{\prime \prime} & \cdot \ell^{*}+\mathcal{J} \vartheta^{\prime \prime} \omega^{*}+\int_{\mathcal{F}(q)}\left(\frac{\partial u_{q^{\prime}}}{\partial t}+\frac{1}{2} \nabla\left|u_{q^{\prime}}\right|^{2}\right) \cdot u^{*} \mathrm{~d} x= \\
& -\int_{\mathcal{F}(q)}\left(\frac{1}{2} \nabla\left|u_{\gamma}\right|^{2}\right) \cdot u^{*} \mathrm{~d} x-\int_{\mathcal{F}(q)}\left(\frac{\partial u_{\gamma}}{\partial t}+\nabla\left(u_{q^{\prime}} \cdot u_{\gamma}\right)\right) \cdot u^{*} \mathrm{~d} x
\end{aligned}
$$

for all $p^{*}:=\left(\omega^{*}, \ell^{*}\right)$ in $\mathbb{R} \times \mathbb{R}^{2}$.

Then the reformulation of Equations (2.1)- $(2.2)$ stated in Theorem 2.2 will follow from the three following lemmas which deal respectively with the left hand side of 5.5 and the two terms in the right hand side. 
Lemma 5.1. For every smooth curve $t \mapsto q(t)$ in $\mathcal{Q}$ and every $p^{*}=\left(\omega^{*}, \ell^{*}\right)$ in $\mathbb{R}^{3}$, one has

$$
\begin{aligned}
m h^{\prime \prime} \cdot \ell^{*}+\mathcal{J} \vartheta^{\prime \prime} \omega^{*}+\int_{\mathcal{F}(q)}\left(\frac{\partial u_{q^{\prime}}}{\partial t}+\right. & \left.\frac{1}{2} \nabla\left|u_{q^{\prime}}\right|^{2}\right) \cdot u^{*} \mathrm{~d} x \\
& =M(q) q^{\prime \prime} \cdot p^{*}+\left\langle\Gamma(q), q^{\prime}, q^{\prime}\right\rangle \cdot p^{*}
\end{aligned}
$$

where $q=(\vartheta, h), u^{*}$ is given by (5.3), $u_{q^{\prime}}$ is given by 2.15a , $M(q)$ and $\Gamma(q)$ are defined in (2.16) and 2.17).

Lemma 5.2. For every $q \in \mathcal{Q}$ and every $p^{*}=\left(\omega^{*}, \ell^{*}\right) \in \mathbb{R}^{3}$,

$$
-\int_{\mathcal{F}(q)}\left(\frac{1}{2} \nabla\left|u_{\gamma}\right|^{2}\right) \cdot u^{*} \mathrm{~d} x=\gamma^{2} E(q) \cdot p^{*},
$$

where $u^{*}$ is given by (5.3), $u_{\gamma}$ is given by 2.15b, $E(q)$ is defined in 2.18b). Lemma 5.3. For every smooth curve $t \mapsto q(t)$ in $\mathcal{Q}$ and every $p^{*}=\left(\omega^{*}, \ell^{*}\right)$ in $\mathbb{R}^{3}$

$$
-\int_{\mathcal{F}(q)}\left(\frac{\partial u_{\gamma}}{\partial t}+\nabla\left(u_{q^{\prime}} \cdot u_{\gamma}\right)\right) \cdot u^{*} \mathrm{~d} x=\gamma\left(q^{\prime} \times B(q)\right) \cdot p^{*},
$$

where $q=(\vartheta, h), u^{*}$ is given by (5.3), $u_{\gamma}$ and $u_{q^{\prime}}$ are given by $(2.15), B(q)$ is defined in 2.18a.

Lemma 5.2 simply follows from an integration by parts. If we consider Lemmas 5.1 and 5.3 as granted, then gathering the results of Lemmas 5.1, 5.2 and 5.3 with (5.5), the conclusion of Theorem 2.2 follows.

Lemmas 5.1 and 5.3 are proved in Subsections 5.2 and 5.3 respectively.

5.2 Reformulation of the potential part: Proof of Lemma 5.1

We start with observing that

$$
m h^{\prime \prime} \cdot \ell^{*}+\mathcal{J} \vartheta^{\prime \prime} \omega^{*}=M_{g} q^{\prime \prime} \cdot p^{*} .
$$

Now in order to deal with the last term of the left hand side of $(5.6)$ we use a Lagrangian strategy. For any $q$ in $\mathcal{Q}$ and every $p$ in $\mathbb{R}^{3}$, let us define

$$
\mathcal{E}_{1}(q, p):=\frac{1}{2} \int_{\mathcal{F}(q)}|\nabla(\varphi(q, \cdot) \cdot p)|^{2} \mathrm{~d} x .
$$

Thus $\mathcal{E}_{1}\left(q, q^{\prime}\right)$ denotes the kinetic energy of the potential part $u_{q^{\prime}}$ of the flow. It follows from classical shape derivative theory (see [6,17, 32, 40, ) that $\mathcal{E}_{1}$ is in $C^{\infty}\left(\mathcal{Q} \times \mathbb{R}^{3} ;[0,+\infty)\right)$.

Now the crucial quantity here is the Euler-Lagrange function:

$$
\mathcal{E} \mathcal{L}=\left(\frac{d}{d t} \frac{\partial \mathcal{E}_{1}}{\partial p}\left(q, q^{\prime}\right)-\frac{\partial \mathcal{E}_{1}}{\partial q}\left(q, q^{\prime}\right)\right) \cdot p^{*}
$$

associated with any smooth curve $t \mapsto q(t)$ in $\mathcal{Q}$ and any $p^{*} \in \mathbb{R}^{3}$. 
Lemma 5.4. For any smooth curve $t \mapsto q(t)$ in $\mathcal{Q}$, for every $p^{*} \in \mathbb{R}^{3}$,

$$
\int_{\mathcal{F}(q)}\left(\frac{\partial u_{q^{\prime}}}{\partial t}+\frac{1}{2} \nabla\left|u_{q^{\prime}}\right|^{2}\right) \cdot u^{*} \mathrm{~d} x=\mathcal{E} \mathcal{L}
$$

where $u_{q^{\prime}}$ is given by (2.15), $u^{*}$ is given by (5.3) and $\mathcal{E} \mathcal{L}$ is given by (5.11).

Proof Let us make use of the following slight abuse of notations which simplifies the presentation of the proof of Lemma 5.4 . For a smooth function $I(q, p)$, where $(q, p)$ is running into $\mathcal{Q} \times \mathbb{R}^{3}$, and a smooth curve $t \mapsto q(t)$ in $\mathcal{Q}$ let us define

$$
\left(\frac{\partial}{\partial q} \frac{d}{d t} I\left(q, q^{\prime}\right)\right)(t):=\left(\frac{\partial}{\partial q} J\right)\left(q(t), q^{\prime}(t), q^{\prime \prime}(t)\right),
$$

where, for $(q, p, r)$ in $\mathcal{Q} \times \mathbb{R}^{3} \times \mathbb{R}^{3}$,

$$
J(q, p, r)=p \frac{\partial I}{\partial q}(q, p)+r \frac{\partial I}{\partial p}(q, p) .
$$

Observe in particular that

$$
\frac{d}{d t}\left(I\left(q(t), q^{\prime}(t)\right)\right)=J\left(q(t), q^{\prime}(t), q^{\prime \prime}(t)\right)
$$

and

$$
\frac{d}{d t}\left(\frac{\partial I}{\partial q}\left(q(t), q^{\prime}(t)\right)\right)=\left(\frac{\partial}{\partial q} \frac{d}{d t} I\left(q, q^{\prime}\right)\right)(t) .
$$

Below, in such circumstances, it will be comfortable to write

$$
\frac{\partial}{\partial q}\left[J\left(q(t), q^{\prime}(t), q^{\prime \prime}(t)\right)\right] \text { instead of }\left(\frac{\partial J}{\partial q}\right)\left(q(t), q^{\prime}(t), q^{\prime \prime}(t)\right)
$$

and it will be understood that $J$ is extended from $\left(q(t), q^{\prime}(t), q^{\prime \prime}(t)\right)$ to general $(q, p, r)$ by (5.13).

Let us also recall the Reynolds transport formula (see [38, pages 12-13]), that we shall use to express the derivative of functionals of the kind:

$$
\mathcal{I}(q)=\int_{\mathcal{F}(q)} f(q, \cdot) \mathrm{d} x,
$$

where $f(q, \cdot)$ is a smooth function defined in $\mathcal{F}(q)$. The formula reads, for every $q \in \mathcal{Q}$ and every $p^{*}$ in $\mathbb{R}^{3}$ :

$$
\frac{\partial \mathcal{I}}{\partial q}(q) \cdot p^{*}=\int_{\mathcal{F}(q)} \frac{\partial f}{\partial q}(q, \cdot) \cdot p^{*} \mathrm{~d} x+\int_{\partial \mathcal{S}(q)} f(q, \cdot)\left(u^{*} \cdot n\right) \mathrm{d} s,
$$

where $u^{*}$ is given by 5.3 .

We start with manipulating the first term of $\mathcal{E} \mathcal{L}$. Upon an integration by parts we arrive at

$$
\frac{\partial \mathcal{E}_{1}}{\partial p} \cdot p^{*}=\int_{\mathcal{F}(q)} u_{q^{\prime}} \cdot u^{*} \mathrm{~d} x=\int_{\partial \mathcal{S}(q)}\left(\boldsymbol{\varphi} \cdot q^{\prime}\right)\left(u^{*} \cdot n\right) \mathrm{d} s .
$$


Then, thanks to formula (5.15),

$$
\frac{\partial \mathcal{E}_{1}}{\partial p} \cdot p^{*}=\frac{\partial}{\partial q}\left(\int_{\mathcal{F}(q)}\left(\boldsymbol{\varphi} \cdot q^{\prime}\right) \mathrm{d} x\right) \cdot p^{*}-\int_{\mathcal{F}(q)}\left(\frac{\partial \varphi}{\partial q} \cdot q^{\prime}\right) \cdot p^{*} \mathrm{~d} x
$$

We differentiate (5.16) with respect to $t$ to arrive at

$$
\begin{aligned}
\frac{d}{d t} \frac{\partial \mathcal{E}_{1}}{\partial p} \cdot p^{*}=\frac{d}{d t} \frac{\partial}{\partial q}\left(\int_{\mathcal{F}(q)}\left(\varphi \cdot q^{\prime}\right) \mathrm{d} x\right) & \cdot p^{*} \\
& -\frac{d}{d t}\left(\int_{\mathcal{F}(q)}\left(\frac{\partial \varphi}{\partial q} \cdot q^{\prime}\right) \cdot p^{*} \mathrm{~d} x\right) .
\end{aligned}
$$

With the abuse of notations mentioned above we exchange the derivatives involved in the first term of the right hand side, so that the identity (5.17) can be rewritten as follows:

$$
\begin{aligned}
\frac{d}{d t} \frac{\partial \mathcal{E}_{1}}{\partial p} \cdot p^{*}=\frac{\partial}{\partial q} \frac{d}{d t}\left(\int_{\mathcal{F}(q)}\left(\varphi \cdot q^{\prime}\right) \mathrm{d} x\right) & \cdot p^{*} \\
& -\frac{d}{d t}\left(\int_{\mathcal{F}(q)}\left(\frac{\partial \varphi}{\partial q} \cdot q^{\prime}\right) \cdot p^{*} \mathrm{~d} x\right) .
\end{aligned}
$$

Moreover, using again the Reynolds transport formula (5.15), we deduce that

$$
\begin{aligned}
\frac{d}{d t}\left(\int_{\mathcal{F}(q)}\left(\boldsymbol{\varphi} \cdot q^{\prime}\right) \mathrm{d} x\right) & =\int_{\mathcal{F}(q)} \frac{\partial}{\partial t}\left(\boldsymbol{\varphi} \cdot q^{\prime}\right) \mathrm{d} x+\int_{\partial \mathcal{S}(q)}\left(\boldsymbol{\varphi} \cdot q^{\prime}\right)\left(u_{q^{\prime}} \cdot n\right) \mathrm{d} s \\
& =\int_{\mathcal{F}(q)} \frac{\partial}{\partial t}\left(\boldsymbol{\varphi} \cdot q^{\prime}\right) \mathrm{d} x+2 \mathcal{E}_{1}\left(q, q^{\prime}\right),
\end{aligned}
$$

by integration by parts.

We infer from (5.18) and (5.19), again with the abuse of notations mentioned above, that:

$$
\begin{aligned}
\mathcal{E} \mathcal{L}=\frac{\partial \mathcal{E}_{1}}{\partial q}+\frac{\partial}{\partial q}\left[\int_{\mathcal{F}(q)} \frac{\partial}{\partial t}\left(\varphi \cdot q^{\prime}\right) \mathrm{d} x\right] & \cdot p^{*} \\
& -\frac{d}{d t}\left(\int_{\mathcal{F}(q)}\left(\frac{\partial \varphi}{\partial q} \cdot q^{\prime}\right) \cdot p^{*} \mathrm{~d} x\right) .
\end{aligned}
$$

Thanks to formula (5.15), we translate the second term of the right hand side into

$$
\begin{aligned}
\frac{\partial}{\partial q}\left[\int_{\mathcal{F}(q)} \frac{\partial}{\partial t}\left(\boldsymbol{\varphi} \cdot q^{\prime}\right) \mathrm{d} x\right] \cdot p^{*}=\int_{\mathcal{F}(q)} & \frac{\partial}{\partial q}\left(\frac{\partial}{\partial t}\left(\boldsymbol{\varphi} \cdot q^{\prime}\right)\right) \cdot p^{*} \mathrm{~d} x \\
& +\int_{\partial \mathcal{S}(q)} \frac{\partial}{\partial t}\left(\boldsymbol{\varphi} \cdot q^{\prime}\right)\left(u^{*} \cdot n\right) \mathrm{d} s
\end{aligned}
$$


and the last one into

$$
\begin{aligned}
\frac{d}{d t}\left(\int_{\mathcal{F}(q)}\left(\frac{\partial \varphi}{\partial q} \cdot q^{\prime}\right) \cdot p^{*} \mathrm{~d} x\right)= & \int_{\mathcal{F}(q)} \frac{\partial}{\partial t}\left(\left(\frac{\partial \varphi}{\partial q} \cdot q^{\prime}\right) \cdot p^{*}\right) \mathrm{d} x \\
& +\int_{\partial \mathcal{S}(q)}\left(\left(\frac{\partial \varphi}{\partial q} \cdot q^{\prime}\right) \cdot p^{*}\right)\left(u_{q^{\prime}} \cdot n\right) \mathrm{d} s
\end{aligned}
$$

Using again (5.14) for the first term and integrating by parts the second one, we arrive at

$$
\begin{aligned}
& \frac{d}{d t}\left(\int_{\mathcal{F}(q)}\left(\frac{\partial \varphi}{\partial q} \cdot q^{\prime}\right) \cdot p^{*} \mathrm{~d} x\right) \\
& \quad=\int_{\mathcal{F}(q)} \frac{\partial}{\partial q}\left(\frac{\partial}{\partial t}\left(\boldsymbol{\varphi} \cdot q^{\prime}\right)\right) \cdot p^{*} \mathrm{~d} x+\int_{\mathcal{F}(q)}\left(\frac{\partial u_{q^{\prime}}}{\partial q} \cdot p^{*}\right) \cdot u_{q^{\prime}} \mathrm{d} x
\end{aligned}
$$

On the other hand, thanks to formula (5.15),

$$
\frac{\partial \mathcal{E}_{1}}{\partial q} \cdot p^{*}=\int_{\mathcal{F}(q)}\left(\frac{\partial u_{q^{\prime}}}{\partial q} \cdot p^{*}\right) \cdot u_{q^{\prime}} \mathrm{d} x+\frac{1}{2} \int_{\partial \mathcal{S}(q)}\left|u_{q^{\prime}}\right|^{2}\left(u^{*} \cdot n\right) \mathrm{d} s .
$$

Substituting the expressions (5.21), (5.23) and (5.24) into (5.20) and simplifying, we end up with:

$$
\mathcal{E} \mathcal{L}=\int_{\partial \mathcal{S}(q)}\left[\frac{\partial}{\partial t}\left(\boldsymbol{\varphi} \cdot q^{\prime}\right)+\frac{1}{2}\left|u_{q^{\prime}}\right|^{2}\right]\left(u^{*} \cdot n\right) \mathrm{d} s
$$

Upon an integration by parts, we recover 5.12 and the proof of Lemma 5.4 is completed.

Now, we observe that the kinetic energy $\mathcal{E}_{1}\left(q, q^{\prime}\right)$ associated with the potential part of the flow, as defined by (5.10), can be rewritten as:

$$
\mathcal{E}_{1}\left(q, q^{\prime}\right)=\frac{1}{2} M_{a}(q) q^{\prime} \cdot q^{\prime}
$$

where $M_{a}(q)$ is defined by (2.16). This allows us to prove the following result.

Lemma 5.5. For any smooth curve $t \mapsto q(t)$ in $\mathcal{Q}$, for every $p^{*} \in \mathbb{R}^{3}$, one has

$$
\mathcal{E} \mathcal{L}=M_{a}(q) q^{\prime \prime} \cdot p^{*}+\left\langle\Gamma(q), q^{\prime}, q^{\prime}\right\rangle \cdot p^{*},
$$

with $\mathcal{E} \mathcal{L}$ is given by (5.11), $M_{a}(q)$ defined by $(2.16)$ and $\Gamma(q)$ associated with $M(q)$ by the Christoffel formula 2.17a $2.17 \mathrm{~b}$. 
Proof of Lemma 5.5. Using 5.25 in the definition 5.11) of $\mathcal{E} \mathcal{L}$, we arrive at

$$
\mathcal{E} \mathcal{L}=M_{a}(q) q^{\prime \prime} \cdot p^{*}+\left(\left(D M_{a}(q) \cdot q^{\prime}\right) q^{\prime}\right) \cdot p^{*}-\frac{1}{2}\left(\left(D M_{a}(q) \cdot p^{*}\right) q^{\prime}\right) \cdot q^{\prime}
$$

Let us recall the notation $\left(M_{a}\right)_{i, j}^{k}(q)$ in $2.17 \mathrm{c}$ and let the notation $\sum$ stand for $\sum_{1 \leqslant i, j, k \leqslant 3}$ for the rest of this proof. Then

$$
\mathcal{E} \mathcal{L}=M_{a} q^{\prime \prime} \cdot p^{*}+\sum\left(M_{a}\right)_{i, j}^{k} q_{k}^{\prime} q_{j}^{\prime} p_{i}^{*}-\frac{1}{2} \sum\left(M_{a}\right)_{i, j}^{k} q_{i}^{\prime} q_{j}^{\prime} p_{k}^{*} .
$$

A symmetrization of the second term of the right hand side above leads to

$$
\begin{aligned}
\mathcal{E} \mathcal{L}= & M_{a} q^{\prime \prime} \cdot p^{*} \\
& +\frac{1}{2}\left(\sum\left(M_{a}\right)_{i, j}^{k} q_{k}^{\prime} q_{j}^{\prime} p_{i}^{*}+\sum\left(M_{a}\right)_{i, k}^{j} q_{k}^{\prime} q_{j}^{\prime} p_{i}^{*}-\sum\left(M_{a}\right)_{i, j}^{k} q_{i}^{\prime} q_{j}^{\prime} p_{k}^{*}\right),
\end{aligned}
$$

Then the result follows by exchanging $i$ and $k$ in the last sum.

Finally Lemma 5.1 straightforwardly results from the combination of (5.9), Lemmas 5.4 and 5.5 This concludes the proof of Lemma 5.1 .

5.3 Reformulation of the cross part: Proof of Lemma 5.3

We now turn to the proof of Lemma 5.3. Write $q:=(\vartheta, h)$ and recall that $\psi(q, \cdot)$ and $C(q)$ are defined in 2.11) and that

$$
u_{\gamma}:=\gamma \nabla^{\perp} \psi(q, \cdot), \quad u_{q^{\prime}}:=\nabla\left(\varphi(q, \cdot) \cdot q^{\prime}\right) \text { and } u^{*}:=\nabla\left(\varphi(q, \cdot) \cdot p^{*}\right) .
$$

Upon an integration by parts, since $\psi(q, \cdot)$ vanishes on $\partial \Omega$,

$$
\int_{\mathcal{F}(q)}\left(\frac{\partial u_{\gamma}}{\partial t}\right) \cdot u^{*} \mathrm{~d} x=-\gamma \int_{\partial \mathcal{S}(q)}\left(\frac{\partial}{\partial t}(\psi(q, \cdot))\right)\left(\frac{\partial \varphi}{\partial \tau}(q, \cdot) \cdot p^{*}\right) \mathrm{d} s .
$$

Lemma 5.6. On $\partial \mathcal{S}(q)$,

$$
\frac{\partial}{\partial t}(\psi(q, \cdot))=-\frac{\partial \psi}{\partial n}(q, \cdot)\left(\frac{\partial \varphi}{\partial n}(q, \cdot) \cdot q^{\prime}\right)+D C(q) \cdot q^{\prime}
$$

Proof We start with the observation that

$$
\frac{\partial}{\partial t}(\psi(q, \cdot))=\frac{\partial \psi}{\partial q}(q, \cdot) \cdot q^{\prime}
$$

is the derivative of the function $\psi(q, \cdot)$ when the boundary $\partial \mathcal{S}(q)$ undergoes a rigid displacement of velocity $w=\omega(x-h)^{\perp}+\ell$ where $q^{\prime}=(\omega, \ell)$. Then we differentiate the identity:

$$
\psi(q, R(\vartheta) X+h)=C(q), \quad \text { for } X \in \partial \mathcal{S}_{0},
$$


with respect to $q$ in the direction $q^{\prime}$. We obtain:

$$
\frac{\partial \psi}{\partial q}(q, x) \cdot q^{\prime}+\nabla \psi(q, x) \cdot w=D C(q) \cdot q^{\prime}, \quad \text { for } x \in \partial \mathcal{S}(q) .
$$

Since $\psi(q, \cdot)$ is constant on $\partial \mathcal{S}(q)$, its tangential derivative is zero. Besides, on $\partial \mathcal{S}(q)$ we have $w \cdot n=u_{q^{\prime}} \cdot n=\frac{\partial \varphi}{\partial n}(q, \cdot) \cdot q^{\prime}$ so

$$
\nabla \psi(q, x) \cdot w=\frac{\partial \psi}{\partial n}(q, x)\left(\frac{\partial \varphi}{\partial n}(q, x) \cdot q^{\prime}\right) \text { for } x \in \partial \mathcal{S}(q) .
$$

Gathering (5.29), 5.30) and (5.31) we obtain 5.28).

Substituting now (5.28) into (5.27), we arrive at

$$
\int_{\mathcal{F}(q)}\left(\frac{\partial u_{\gamma}}{\partial t}\right) \cdot u^{*} \mathrm{~d} x=\gamma \int_{\partial \mathcal{S}(q)} \frac{\partial \psi}{\partial n}\left(\frac{\partial \varphi}{\partial n} \cdot q^{\prime}\right)\left(\frac{\partial \varphi}{\partial \tau} \cdot p^{*}\right) \mathrm{d} s .
$$

On the other hand, by an integration by parts,

$$
\int_{\mathcal{F}(q)} \nabla\left(u_{q^{\prime}} \cdot u_{\gamma}\right) \cdot u^{*} \mathrm{~d} x=\gamma \int_{\partial \mathcal{S}(q)}\left(u_{q^{\prime}} \cdot u_{\gamma}\right)\left(u^{*} \cdot n\right) \mathrm{d} s .
$$

Adding 5.32 and 5.33) and using that $u_{\gamma}=-\gamma \frac{\partial \psi}{\partial n} \tau$ on $\partial \mathcal{S}(q)$, we arrive at

$$
\begin{aligned}
\int_{\mathcal{F}(q)}( & \left.\frac{\partial u_{\gamma}}{\partial t}+\nabla\left(u_{q^{\prime}} \cdot u_{\gamma}\right)\right) \cdot u^{*} \mathrm{~d} x= \\
& \gamma \int_{\partial \mathcal{S}(q)} \frac{\partial \psi}{\partial n}\left[\left(\frac{\partial \varphi}{\partial n} \cdot q^{\prime}\right)\left(\frac{\partial \varphi}{\partial \tau} \cdot p^{*}\right)-\left(\frac{\partial \varphi}{\partial n} \cdot p^{*}\right)\left(\frac{\partial \varphi}{\partial \tau} \cdot q^{\prime}\right)\right] \mathrm{d} s
\end{aligned}
$$

which is 5.8). This ends the proof of Lemma 5.3 .

\subsection{Decomposition of the Christoffel symbols: Proof of Proposition 2.8}

This subsection is devoted to the proof of Proposition 2.8. We will use the matrix $\underline{M}_{a}(q)$ given by

$$
\underline{M}_{a}(q):=\mathcal{R}(\vartheta)^{t} M_{a}(q) \mathcal{R}(\vartheta),
$$

where we recall that $\mathcal{R}(\vartheta)$ is the rotation matrix defined by 4.11). We also introduce the following real-valued functions depending on the variables $q=$ $(\vartheta, h) \in \mathcal{Q}, p$ in $\mathbb{R}^{3}$ and $p^{*}$ in $\mathbb{R}^{3}$ :

$$
\begin{aligned}
& \Xi_{1}\left(q, p, p^{*}\right):=\left[\left(\frac{\partial \underline{M}_{a}}{\partial q}(q) \cdot p^{*}\right) \mathcal{R}(\vartheta)^{t} p\right] \cdot \mathcal{R}(\vartheta)^{t} p, \\
& \Xi_{3}\left(q, p, p^{*}\right):=\left[\left(\frac{\partial \underline{M}_{a}}{\partial q}(q) \cdot p\right) \mathcal{R}(\vartheta)^{t} p\right] \cdot \mathcal{R}(\vartheta)^{t} p^{*} .
\end{aligned}
$$


The indices in $\Xi_{1}$ and $\Xi_{3}$ above are chosen in order to recall the position where $p^{*}$ appears (the "first $p$ " in the expression of $\Xi_{1}$ is $p^{*}$ and so on.) Similarly we define, for $p=(\omega, \ell)$ and $p^{*}=\left(\omega^{*}, \ell^{*}\right)$ the functions:

$$
\begin{array}{r}
\Upsilon_{1}\left(q, p, p^{*}\right):=\omega^{*} M_{a}(q) p \cdot\left(\begin{array}{c}
0 \\
\ell^{\perp}
\end{array}\right), \quad \Upsilon_{2}\left(q, p, p^{*}\right):=\omega M_{a}(q) p^{*} \cdot\left(\begin{array}{c}
0 \\
\ell^{\perp}
\end{array}\right) \\
\text { and } \Upsilon_{3}\left(q, p, p^{*}\right):=\omega M_{a}(q) p \cdot\left(\begin{array}{c}
0 \\
\ell^{* \perp}
\end{array}\right) .
\end{array}
$$

The proof of Proposition 2.8 is then split into the proof of the following three ancillary lemmas.

Lemma 5.7. For any $\left(q, p, p^{*}\right)$ in $\mathcal{Q} \times \mathbb{R}^{3} \times \mathbb{R}^{3}$,

$$
\begin{aligned}
\langle\Gamma(q), p, p\rangle \cdot p^{*}=\Upsilon_{1}\left(q, p, p^{*}\right)-\Upsilon_{2}(q, p & \left., p^{*}\right)-\Upsilon_{3}\left(q, p, p^{*}\right) \\
& +\Xi_{3}\left(q, p, p^{*}\right)-\frac{1}{2} \Xi_{1}\left(q, p, p^{*}\right) .
\end{aligned}
$$

Lemma 5.8. For any $\left(q, p, p^{*}\right)$ in $\mathcal{Q} \times \mathbb{R}^{3} \times \mathbb{R}^{3}$,

$$
\Upsilon_{1}\left(q, p, p^{*}\right)-\Upsilon_{2}\left(q, p, p^{*}\right)-\Upsilon_{3}\left(q, p, p^{*}\right)=\left\langle\Gamma^{\mathrm{rot}}(q), p, p\right\rangle \cdot p^{*},
$$

where $\Gamma^{\mathrm{rot}}(q)$ is defined in 2.26 .

Lemma 5.9. For any $\left(q, p, p^{*}\right)$ in $\mathcal{Q} \times \mathbb{R}^{3} \times \mathbb{R}^{3}$,

$$
\Xi_{3}\left(q, p, p^{*}\right)-\frac{1}{2} \Xi_{1}\left(q, p, p^{*}\right)=\left\langle\Gamma^{\partial \Omega}(q), p, p\right\rangle \cdot p^{*},
$$

where $\Gamma^{\partial \Omega}(q)$ is defined in 2.27).

Before proving these three lemmas, we introduce a few notations. For every $q=(\vartheta, h) \in \mathcal{Q}$, we define the change of variables $y=R(\vartheta)^{t}(x-h)$, the domains

$$
\underline{\Omega}(q):=R(\vartheta)^{t}(\Omega-h), \quad \underline{\mathcal{F}}(q):=R(\vartheta)^{t}(\mathcal{F}(q)-h)=\underline{\Omega}(q) \backslash \mathcal{S}_{0},
$$

and the functions $\underline{\varphi}_{i}(q, y)$ such that

$$
\underline{\varphi}(q, \cdot):=\left(\underline{\varphi}_{1}(q, \cdot), \underline{\varphi}_{2}(q, \cdot), \underline{\varphi}_{3}(q, \cdot)\right),
$$

satisfies

$$
\underline{\varphi}(q, y)=\mathcal{R}(\vartheta)^{t} \boldsymbol{\varphi}(q, x), \quad y \in \underline{\mathcal{F}}(q) .
$$

For every $j=1,2,3$, the functions $\underline{\varphi}_{j}(q, \cdot)$ are harmonic in $\underline{\mathcal{F}}(q)$ and satisfy:

$$
\begin{aligned}
& \frac{\partial \underline{\varphi}_{j}}{\partial n}(q, y)=\left\{\begin{array}{ll}
y^{\perp} \cdot n & j=1 ; \\
n_{j-1} & j=2,3
\end{array} \quad \text { on } \partial \mathcal{S}_{0},\right. \\
& \frac{\partial \underline{\varphi}_{j}}{\partial n}(q, y)=0 \quad(j=1,2,3) \quad \text { on } \partial \underline{\Omega}(q) .
\end{aligned}
$$

Therefore the matrix $\underline{M}_{a}(q)$ defined in 5.34 can be recast as

$$
\underline{M}_{a}(q)=\int_{\partial \mathcal{S}_{0}} \underline{\varphi}(q) \otimes \frac{\partial \underline{\varphi}}{\partial n}(q) \mathrm{d} s .
$$


Proof of Lemma 5.7. Let $t \mapsto q(t)$ be a smooth curve in $\mathcal{Q}$, defined in a neighborhood of 0 such that $q(0)=q$ and $q^{\prime}(0)=p$. On the one hand, it follows from (5.25) and (5.34) that for every $p^{*}=\left(\omega^{*}, \ell^{*}\right) \in \mathbb{R}^{3}$,

$$
\frac{d}{d t} \frac{\partial \mathcal{E}_{1}}{\partial p} \cdot p^{*}=M_{a}(q) p^{\prime} \cdot p^{*}-\Upsilon_{2}\left(q, p, p^{*}\right)+\Xi_{3}\left(q, p, p^{*}\right)-\Upsilon_{3}\left(q, p, p^{*}\right) .
$$

On the other hand,

$$
\frac{\partial \mathcal{E}_{1}}{\partial q} \cdot p^{*}=-\Upsilon_{1}\left(q, p, p^{*}\right)+\frac{1}{2} \Xi_{1}\left(q, p, p^{*}\right) .
$$

Gathering (5.11), (5.26), (5.39) and (5.40), we deduce (5.35).

Proof of Lemma 5.8. On the one hand, since $M(q)$ is symmetric,

$$
\Upsilon_{2}\left(q, p, p^{*}\right)=\omega M_{a}(q)\left(\begin{array}{c}
0 \\
\ell^{\perp}
\end{array}\right) \cdot p^{*} .
$$

On the other hand, with the notation of 2.25,

$$
-\Upsilon_{3}\left(q, p, p^{*}\right)+\Upsilon_{1}\left(q, p, p^{*}\right)=\left(\begin{array}{c}
-P_{a}^{\perp} \cdot \ell \\
\omega P_{a}^{\perp}
\end{array}\right) \cdot p^{*}=-\left[\left(\begin{array}{c}
0 \\
P_{a}
\end{array}\right) \times p\right] \cdot p^{*} .
$$

Now gathering (5.41) and (5.42) we conclude the proof of Lemma 5.8

Proof of Lemma 5.9. We will use the following lemma.

Lemma 5.10. For $i, j=1,2,3$, for every $\hat{q}=(\hat{\vartheta}, \hat{h})$ in $\mathcal{Q}$ and every $p^{*}=$ $\left(\omega^{*}, \ell^{*}\right)$ in $\mathbb{R}^{3}$,

$$
\left.\frac{\partial}{\partial q}\left(\int_{\underline{\mathcal{F}}(q)} \nabla \underline{\varphi}_{i}(q) \cdot \nabla \underline{\varphi}_{j}(q) \mathrm{d} y\right)\right|_{q=\hat{q}} \cdot p^{*}=-\int_{\partial \underline{\Omega}(\hat{q})} \frac{\partial \underline{\varphi}_{i}}{\partial \tau} \frac{\partial \underline{\varphi}_{j}}{\partial \tau}\left(\hat{w}^{*} \cdot n\right) \mathrm{d} s
$$

with

$$
\hat{w}^{*}\left(\hat{q}, p^{*}, \cdot\right):=-\omega^{*} \cdot{ }^{\perp}-R(\hat{\vartheta})^{t} \ell^{*} .
$$

Let us first take Lemma 5.10 for granted and conclude the proof of Lemma 5.9 , Applying the change of variables $x=R(\hat{\vartheta}) y+\hat{h}$, we deduce that:

$$
\begin{aligned}
\frac{\partial \underline{M}_{a}}{\partial q}(\hat{q}) \cdot p^{*} & =\left(\left.\frac{\partial}{\partial q}\left(\int_{\underline{\mathcal{F}}(q)} \nabla \underline{\varphi}_{i}(q) \cdot \nabla \underline{\varphi}_{j}(q) \mathrm{d} y\right)\right|_{q=\hat{q}} \cdot p^{*}\right)_{1 \leqslant i, j \leqslant 3} \\
& =-\left(\int_{\partial \underline{\Omega}(\hat{q})} \frac{\partial \underline{\varphi}_{i}}{\partial \tau} \frac{\partial \underline{\varphi}_{j}}{\partial \tau}\left(\hat{w}^{*} \cdot n\right) \mathrm{d} s\right)_{1 \leqslant i, j \leqslant 3} \\
& =\mathcal{R}(\hat{\vartheta})^{t}\left(\int_{\partial \Omega} \frac{\partial \varphi_{i}}{\partial \tau}(\hat{q}) \frac{\partial \varphi_{j}}{\partial \tau}(\hat{q})\left(w^{*} \cdot n\right) \mathrm{d} s\right)_{1 \leqslant i, j \leqslant 3} \mathcal{R}(\hat{\vartheta}),
\end{aligned}
$$


with $w^{*}:=\omega^{*}(x-h)^{\perp}+\ell^{*}$. Therefore, applying this with $\left(\hat{q}, p^{*}\right)=\left(q, p^{*}\right)$ and with $\left(\hat{q}, p^{*}\right)=(q, p)$, we arrive at

$$
\Xi_{3}\left(q, p, p^{*}\right)-\frac{1}{2} \Xi_{1}\left(q, p, p^{*}\right)=\sum\left[\Lambda_{i j}^{l}(q) p_{l} p_{j}-\frac{1}{2} \Lambda_{j l}^{i}(q) p_{l} p_{j}\right] p_{i}^{*},
$$

where as before, the notation $\sum$ stands for $\sum_{1 \leqslant i, j, l \leqslant 3}$ in this proof and where for $k=1,2,3$, the matrices $\Lambda^{k}(\hat{q})$ are given by:

$$
\Lambda^{k}(\hat{q})=\left(\int_{\partial \Omega} \frac{\partial \varphi_{i}}{\partial \tau}(\hat{q}) \frac{\partial \varphi_{j}}{\partial \tau}(\hat{q}) K_{k}(\hat{q}, \cdot) \mathrm{d} s\right)_{1 \leqslant i, j \leqslant 3} .
$$

We recall that $K_{1}(\hat{q}, \cdot)=(x-\hat{h})^{\perp} \cdot n$ and $K_{j}(\hat{q}, \cdot)=n_{j-1}(j=2,3)$ on $\partial \Omega$. Now the quadratic form in $p$ can be symmetrized as follows:

$$
\sum\left[\Lambda_{i j}^{l}(q) p_{l} p_{j}-\frac{1}{2} \Lambda_{j l}^{i}(q) p_{l} p_{j}\right] p_{i}^{*}=\frac{1}{2} \sum\left[\Lambda_{i j}^{l}+\Lambda_{i l}^{j}-\Lambda_{j l}^{i}\right](q) p_{l} p_{j} p_{i}^{*},
$$

which leads to 5.36. This concludes the proof of Lemma 5.9

Now we give the proof of Lemma 5.10 .

Proof of Lemma 5.10. The quantity

$$
\left.\frac{\partial}{\partial q}\left(\int_{\underline{\mathcal{F}}(q)} \nabla \underline{\varphi}_{i}(q) \cdot \nabla \underline{\varphi}_{j}(q) \mathrm{d} y\right)\right|_{q=\hat{q}} \cdot p^{*}
$$

can be interpreted as the time derivative of the quantity between parentheses, when the outer boundary $\partial \underline{\Omega}(\hat{q})$ undergoes a rigid displacement of velocity $w^{*}$.

More precisely, denote by $\chi$ a cut-off function, compactly supported, valued in $[0,1]$ and such that $\chi=1$ in a neighborhood of $\partial \underline{\Omega}(\hat{q})$ and $\chi=0$ in a neighborhood of $\mathcal{S}_{0}$. Then, denote by $\xi(t, \cdot)$ the flow associated with the ODE:

$$
\xi^{\prime}(t, y)=\chi(\xi(t, y)) \hat{w}^{*}(t, \xi(t, y)), \quad \text { for } t>0, \text { with } \xi(0, y)=y,
$$

where $\hat{w}^{*}$ was introduced in (5.44). Notice that:

$$
\xi(t, y)=R\left(-t \omega^{*}\right) y-t R(\hat{\vartheta})^{t} \ell^{*},
$$

in a neighborhood of $\partial \underline{\Omega}(\hat{q})$ and $\xi(t, y)=y$ in a neighborhood of $\partial \mathcal{S}_{0}$.

For every $t$ small, define

$$
\underline{\Omega}_{t}:=\xi(t, \underline{\Omega}(\hat{q})) \text { and } \underline{\mathcal{F}}_{t}:=\xi(t, \underline{\mathcal{F}}(\hat{q})) .
$$

For $j=1,2,3$, let $\underline{\varphi}_{j, t}$ be harmonic in $\underline{\mathcal{F}}_{t}$ and satisfy the Neumann boundary conditions:

$$
\begin{aligned}
& \frac{\partial \underline{\varphi}_{j, t}}{\partial n}=\left\{\begin{array}{ll}
(y-\hat{h})^{\perp} \cdot n & j=1 ; \\
n_{j-1} & j=2,3
\end{array} \quad \text { on } \partial \mathcal{S}_{0},\right. \\
& \frac{\partial \underline{\varphi}_{j, t}}{\partial n}=0 \quad(j=1,2,3) \quad \text { on } \partial \underline{\Omega}_{t} \text {. }
\end{aligned}
$$


With these settings, the quantity 5.45 can be rewritten as:

$$
\left.\frac{d}{d t}\left(\int_{\underline{\mathcal{F}}_{t}} \nabla \underline{\varphi}_{i, t} \cdot \nabla \underline{\varphi}_{j, t} \mathrm{~d} x\right)\right|_{t=0}
$$

According to the Reynolds transport formula (5.15), it can be expanded as follows:

$$
\begin{array}{r}
\left.\frac{d}{d t}\left(\int_{\underline{\mathcal{F}}_{t}} \nabla \underline{\varphi}_{i, t} \cdot \nabla \underline{\varphi}_{j, t} \mathrm{~d} x\right)\right|_{t=0}=\int_{\underline{\mathcal{F}(\hat{q})}} \nabla \underline{\varphi}_{i}^{\prime} \cdot \nabla \underline{\varphi}_{j} \mathrm{~d} x+\int_{\underline{\mathcal{F}}(\hat{q})} \nabla \underline{\varphi}_{i} \cdot \nabla \underline{\varphi}_{j}^{\prime} \mathrm{d} x \\
+\int_{\partial \underline{\Omega}(\hat{q})}\left(\nabla \underline{\varphi}_{i} \cdot \nabla \underline{\varphi}_{j}\right)\left(\hat{w}^{*} \cdot n\right) \mathrm{d} s,
\end{array}
$$

where

$$
\underline{\varphi}_{j}^{\prime}:=\left.\frac{\partial \underline{\varphi}_{j, t}}{\partial t}\right|_{t=0} .
$$

Lemma 5.11. For $j=1,2,3$, the function $\underline{\varphi}_{j}^{\prime}$ is harmonic in $\underline{\mathcal{F}}(\hat{q})$, satisfies

$$
\frac{\partial \underline{\varphi}_{j}^{\prime}}{\partial n}=0 \text { on } \partial \mathcal{S}_{0}
$$

and

$$
\frac{\partial \underline{\varphi}_{j}^{\prime}}{\partial n}=\frac{\partial}{\partial \tau}\left(\left(\hat{w}^{*} \cdot n\right) \frac{\partial \underline{\varphi}_{j}}{\partial \tau}\right) \text { on } \partial \underline{\Omega}(\hat{q}) .
$$

Once Lemma 5.11 is proved, (5.43) follows from $(5.48$ and an integration by parts.

Proof of Lemma 5.11. The function $\underline{\varphi}_{j}^{\prime}$ is defined and harmonic in $\underline{\mathcal{F}}(\hat{q})$ and the boundary conditions are obtained by differentiating with respect to $t$, at $t=0$, the identities on the fixed boundaries $\partial \mathcal{S}_{0}$ and $\partial \underline{\Omega}(\hat{q})$ :

$$
\begin{array}{ll}
\frac{\partial \underline{\varphi}_{j, t}}{\partial n}(\xi(t, \cdot))= \begin{cases}(y-\hat{h})^{\perp} \cdot n & j=1 ; \\
n_{j-1} & j=2,3 ;\end{cases} & \text { on } \partial \mathcal{S}_{0}, \\
\frac{\partial \underline{\varphi}_{j, t}}{\partial n}(\xi(t, \cdot))=0 \quad(j=1,2,3) & \text { on } \partial \underline{\Omega}(\hat{q}) .
\end{array}
$$

Let us focus on the proof of 5.50 , the proof of 5.49 being quite similar with some simplifications. On $\partial \underline{\Omega}(\hat{q})$, using $(5.46)$, we can write that:

$$
\left.\frac{d}{d t}\left(\frac{\partial \underline{\varphi}_{j, t}}{\partial n}(\xi(t, \cdot))\right)\right|_{t=0}=\frac{\partial \underline{\varphi}_{j}^{\prime}}{\partial n}+\left\langle D^{2} \underline{\varphi}_{j}, \hat{w}^{*}, n\right\rangle+\omega^{*} \frac{\partial \underline{\varphi}_{j}}{\partial \tau}
$$


where the last term is obtained by noticing that $n(\xi(t, \cdot))=R\left(-t \omega^{*}\right) n$. Therefore by taking the derivative at $t=0$ of the identity (5.51b) and using (5.52) we obtain

$$
\begin{aligned}
\frac{\partial \underline{\varphi}_{j}^{\prime}}{\partial n} & =-\left\langle D^{2} \underline{\varphi}_{j}, \hat{w}^{*}, n\right\rangle-\omega^{*} \frac{\partial \underline{\varphi}_{j}}{\partial \tau} \\
& =-\frac{\partial^{2} \underline{\varphi}_{j}}{\partial n^{2}}\left(\hat{w}^{*} \cdot n\right)-\left\langle D^{2} \underline{\varphi}_{j}, \tau, n\right\rangle\left(\hat{w}^{*} \cdot \tau\right)-\omega^{*} \frac{\partial \underline{\varphi}_{j}}{\partial \tau}
\end{aligned}
$$

by decomposing $w^{*}$ into normal and tangential parts. Taking the tangential derivative of the identity $5.37 \mathrm{~b}$ and using the relation $\frac{\partial n}{\partial \tau}=\kappa \tau$ with $\kappa$ the local curvature of $\partial \underline{\Omega}(\hat{q})$, we arrive at

$$
\left\langle D^{2} \underline{\varphi}_{j}, \tau, n\right\rangle+\kappa \frac{\partial \underline{\varphi}^{j}}{\partial \tau}=0 \quad \text { on } \partial \underline{\Omega}(\hat{q}) .
$$

We substitute 5.54 into 5.53 to deduce:

$$
\begin{aligned}
\frac{\partial \underline{\varphi}_{j}^{\prime}}{\partial n} & =-\frac{\partial^{2} \underline{\varphi}_{j}}{\partial n^{2}}\left(\hat{w}^{*} \cdot n\right)+\left(\kappa\left(\hat{w}^{*} \cdot \tau\right)-\omega^{*}\right) \frac{\partial \underline{\varphi}_{j}}{\partial \tau} \\
& =-\frac{\partial^{2} \underline{\varphi}_{j}}{\partial n^{2}}\left(\hat{w}^{*} \cdot n\right)+\left(\frac{\partial}{\partial \tau}\left(\hat{w}^{*} \cdot n\right)\right) \frac{\partial \underline{\varphi}_{j}}{\partial \tau} .
\end{aligned}
$$

On $\partial \underline{\Omega}(\hat{q})$, we have with local coordinates:

$$
\Delta \underline{\varphi}_{j}=\frac{\partial}{\partial \tau}\left(\frac{\partial \underline{\varphi}_{j}}{\partial \tau}\right)+\kappa \frac{\partial \underline{\varphi}_{j}}{\partial n}+\frac{\partial^{2} \underline{\varphi}_{j}}{\partial n^{2}}
$$

Since $\underline{\varphi}_{j}$ is harmonic and $\frac{\partial \underline{\varphi}_{j}}{\partial n}=0$ on $\partial \underline{\Omega}(\hat{q})$, we deduce that $\frac{\partial^{2} \underline{\varphi}_{j}}{\partial n^{2}}=-\frac{\partial^{2} \underline{\varphi}_{j}}{\partial \tau^{2}}$ on $\partial \underline{\Omega}(\hat{q})$, and therefore

$$
\frac{\partial \underline{\varphi}_{j}^{\prime}}{\partial n}=\left(\hat{w}^{*} \cdot n\right) \frac{\partial}{\partial \tau}\left(\frac{\partial \underline{\varphi}_{j}}{\partial \tau}\right)+\left(\frac{\partial}{\partial \tau}\left(\hat{w}^{*} \cdot n\right)\right) \frac{\partial \underline{\varphi}_{j}}{\partial \tau},
$$

which is 5.50 .

5.5 Identification of the electric potential: Proof of Lemma 2.5

We now establish Lemma 2.5. By definition,

$$
C(q)=-\int_{\mathcal{F}(q)} \nabla \psi(q, \cdot) \cdot \nabla \psi(q, \cdot) \mathrm{d} x .
$$

Thus, by Reynolds transport formula 5.15 we infer that for every $p$ in $\mathbb{R}^{3}$,

$$
D C(q) \cdot p=-2 \int_{\mathcal{F}(q)} \nabla\left(\frac{\partial \psi}{\partial q} \cdot p\right) \cdot \nabla \psi \mathrm{d} x-\int_{\partial \mathcal{S}(q)}|\nabla \psi|^{2} u_{q^{\prime}} \cdot n \mathrm{~d} s
$$


where $u_{q^{\prime}}$ was defined in 2.15. Upon an integration by parts,

$$
\int_{\mathcal{F}(q)} \nabla\left(\frac{\partial \psi}{\partial q} \cdot p\right) \cdot \nabla \psi \mathrm{d} x=\int_{\partial \mathcal{S}(q)}\left(\frac{\partial \psi}{\partial q} \cdot p\right) \frac{\partial \psi}{\partial n} \mathrm{~d} s .
$$

Moreover gathering (5.30) and (5.31) we arrive at

$$
\frac{\partial \psi}{\partial q}(q, x) \cdot p=D C(q) \cdot p-\frac{\partial \psi}{\partial n}(q, x) \frac{\partial \varphi}{\partial n}(q, x) \cdot p . \quad \text { for } x \in \partial \mathcal{S}(q) .
$$

Now combining (5.57) and 5.58) we obtain

$$
\begin{aligned}
\int_{\mathcal{F}(q)} \nabla\left(\frac{\partial \psi}{\partial q} \cdot p\right) \cdot \nabla \psi \mathrm{d} x & =\int_{\partial \mathcal{S}(q)}(D C(q) \cdot p) \frac{\partial \psi}{\partial n} \mathrm{~d} s-\int_{\partial \mathcal{S}(q)}\left|\frac{\partial \psi}{\partial n}\right|^{2} \frac{\partial \varphi}{\partial n} \cdot p \mathrm{~d} s \\
& =-D C(q) \cdot p-\int_{\partial \mathcal{S}(q)}\left|\frac{\partial \psi}{\partial n}\right|^{2} \frac{\partial \varphi}{\partial n} \cdot p \mathrm{~d} s,
\end{aligned}
$$

thanks to 2.11d . On the other hand since $\psi(q, \cdot)$ is constant on $\partial \mathcal{S}(q)$,

$$
\int_{\partial \mathcal{S}(q)}|\nabla \psi|^{2} u_{q^{\prime}} \cdot n \mathrm{~d} s=\int_{\partial \mathcal{S}(q)}\left|\frac{\partial \psi}{\partial n}\right|^{2} \frac{\partial \varphi}{\partial n} \cdot p \mathrm{~d} s .
$$

Gathering (5.56), (5.59) and (5.60) and recalling the definition (2.18b) of $E$, we obtain (2.23). This concludes the proof of Lemma 2.5 .

5.6 Conservation of energy: Proof of Proposition 2.6

We prove Proposition 2.6. We start with the observation that

$$
\left(\mathcal{E}\left(q, q^{\prime}\right)\right)^{\prime}=M(q) q^{\prime \prime} \cdot q^{\prime}+\frac{1}{2}\left(D M(q) \cdot q^{\prime}\right) q^{\prime} \cdot q^{\prime}-\frac{1}{2} \gamma^{2} D C(q) \cdot q^{\prime} .
$$

Now, thanks to 2.21) and 2.18c),

$$
M(q) q^{\prime \prime} \cdot q^{\prime}=-\left\langle\Gamma(q), q^{\prime}, q^{\prime}\right\rangle \cdot q^{\prime}+F\left(q, q^{\prime}\right) \cdot q^{\prime},
$$

and

$$
F\left(q, q^{\prime}\right) \cdot q^{\prime}=\gamma^{2} E(q) \cdot q^{\prime}
$$

We introduce the matrix for any $(q, p)$ in $\mathcal{Q} \times \mathbb{R}^{3}$,

$$
S(q, p):=\left(\sum_{1 \leqslant i \leqslant 3} \Gamma_{i, j}^{k}(q) p_{i}\right)_{1 \leqslant k, j \leqslant 3},
$$

so that

$$
\langle\Gamma(q), p, p\rangle=S(q, p) p
$$


Combining (5.61), 5.62, 5.63, 5.64) and 5.65) we obtain

$$
\left(\mathcal{E}\left(q, q^{\prime}\right)\right)^{\prime}=\gamma^{2}\left(E(q)-\frac{1}{2} D C(q)\right) \cdot q^{\prime}+\left(\frac{1}{2} D M(q) \cdot q^{\prime}-S\left(q, q^{\prime}\right)\right) q^{\prime} \cdot q^{\prime}
$$

The first term of the right hand side vanishes thanks to Lemma 2.5. Proposition 2.6 follows then from the following result.

Lemma 5.12. For any $(q, p)$ in $\mathcal{Q} \times \mathbb{R}^{3}$, the matrix $\frac{1}{2} D M(q) \cdot p-S(q, p)$ is skew-symmetric.

Proof We first observe that $D M(q) \cdot p$ is the $3 \times 3$ matrix containing the entries

$$
\sum_{1 \leqslant k \leqslant 3}\left(M_{a}\right)_{i, j}^{k}(q) p_{k}, \text { for } 1 \leqslant i, j \leqslant 3
$$

where $\left(M_{a}\right)_{i, j}^{k}(q)$ is defined in $2.17 \mathrm{c}$. On the other hand, the matrix $S(q, p)$ contains the entries

$$
\frac{1}{2} \sum_{1 \leqslant k \leqslant 3}\left(\left(M_{a}\right)_{i, j}^{k}+\left(M_{a}\right)_{i, k}^{j}-\left(M_{a}\right)_{k, j}^{i}\right)(q) p_{k}
$$

for $1 \leqslant i, j \leqslant 3$. Therefore, the matrix $D M(q) \cdot p-S(q, p)$ contains the entries

$$
c_{i j}(q, p)=-\frac{1}{2} \sum_{1 \leqslant k \leqslant 3}\left(\left(M_{a}\right)_{i, k}^{j}-\left(M_{a}\right)_{k, j}^{i}\right)(q) p_{k},
$$

for $1 \leqslant i, j \leqslant 3$. Since the matrix $M(q)$ is symmetric, $c_{i j}(q, p)=-c_{j i}(q, p)$ for $1 \leqslant i, j \leqslant 3$.

5.7 The case of a disk: proof of Theorem 2.9

In this subsection, we suppose that $\mathcal{S}_{0}$ is a disk, of center $\zeta$ and radius $r_{0}$. We start by observing that, as noticed in Subsection 4.1 $\zeta=h_{c}(0)=h_{c}(0)-h(0)$, so with 4.15) we deduce $\zeta_{\vartheta}=h_{c}-h$, that is, 2.35b). Relation (2.35c is an immediate consequence of the decomposition $x-h=\left(x-h_{c}\right)+\left(h_{c}-h\right)$, of the fact that $\left(x-h_{c}\right) \cdot n=0$ on $\partial \mathcal{S}(t)$, and of $\left.2.1 \mathrm{c}-2.1 \mathrm{~d}\right)$. Hence only 2.35a needs proving.

Concerning the added mass matrix, due to $(2.9)$, in this case, $\partial_{n} \varphi_{1}(q, \cdot)=$ $\left(h_{c}-h\right)^{\perp} \cdot n(\cdot)$, and consequently,

$$
\varphi_{1}(q, \cdot)=-\zeta_{\vartheta, 2} \varphi_{2}(q, \cdot)+\zeta_{\vartheta, 1} \varphi_{3}(q, \cdot) .
$$

We underline that $\varphi_{2}$ and $\varphi_{3}$ depend merely on $h_{c}$ while $\varphi_{1}$ depends also on $\vartheta$. From (5.67), one deduces that for any $q$ in $\mathcal{Q}$ such that $d\left(B\left(h, r_{0}\right), \partial \Omega\right)>0$ and any $p=(\omega, \ell)$ :

$$
M_{a}(q) p \cdot p=\tilde{M}_{b}\left(h_{c}\right) p_{b} \cdot p_{b}
$$


with

$$
p_{b}=p_{b}(\vartheta, \omega, \ell):=\ell+\omega \zeta_{\vartheta}^{\perp} .
$$

Notice that for a solution $(p, q):[0, T] \rightarrow \mathcal{Q} \times \mathbb{R}^{3}$ of the system with $p=q^{\prime}$, for all times,

$$
p_{b}\left(\vartheta(t), \vartheta^{\prime}(t), h^{\prime}(t)\right)=h_{c}^{\prime}(t) .
$$

Now to establish 2.35a we rely on the following adaptation of Lemma 5.5.

Lemma 5.13. For any smooth curve $t \mapsto q(t)$ in $\mathcal{Q}$, for every $p^{*}=\left(\omega^{*}, \ell^{*}\right) \in$ $\mathbb{R}^{3}$,

$$
\mathcal{E} \mathcal{L}=\tilde{M}_{b}\left(h_{c}\right) p_{b}^{\prime} \cdot p_{b}^{*}+\left\langle\Gamma_{b}\left(h_{c}\right), p_{b}, p_{b}\right\rangle \cdot p_{b}^{*},
$$

where

$$
p_{b}^{*}=p_{b}^{*}\left(\vartheta, \omega^{*}, \ell^{*}\right):=\ell^{*}+\omega^{*} \zeta_{\vartheta}^{\perp},
$$

with $\mathcal{E L}$ is given by (5.11), $\tilde{M}_{b}\left(h_{c}\right)$ is defined by 2.29)-(2.31) and $\Gamma_{b}\left(h_{c}\right)$ is defined in 2.32 .

Proof Mimicking the proof of Lemma 5.5 using $\frac{d}{d t} p_{b}^{*}=-\vartheta^{\prime} \omega^{*} \zeta_{\vartheta}, 2.31$ and (5.68) we obtain

$$
\frac{d}{d t} \frac{\partial \mathcal{E}_{1}}{\partial p} \cdot p^{*}=\tilde{M}_{b} p_{b}^{\prime} \cdot p_{b}^{*}+\left(D_{h_{c}} \tilde{M}_{b} \cdot h_{c}^{\prime}\right) p_{b} \cdot p_{b}^{*}-\tilde{M}_{b} p_{b} \cdot\left(\vartheta^{\prime} \omega^{*} \zeta_{\vartheta}\right) .
$$

On the other hand,

$$
\frac{\partial \mathcal{E}_{1}}{\partial q} \cdot p^{*}=\frac{1}{2}\left(D_{h_{c}} \tilde{M}_{b} \cdot \omega^{*} \zeta_{\vartheta}^{\perp}\right) p_{b} \cdot p_{b}+\tilde{M}_{b}\left(\vartheta^{\prime} \omega^{*} \zeta_{\vartheta}\right) \cdot p_{b} .
$$

We deduce that

$$
\mathcal{E} \mathcal{L}=\tilde{M}_{b} p_{b}^{\prime} \cdot p_{b}^{*}+\left(D_{h_{c}} \tilde{M}_{b} \cdot h_{c}^{\prime}\right) p_{b} \cdot p_{b}^{*}-\frac{1}{2}\left(D_{h_{c}} \tilde{M}_{b} \cdot \omega^{*} \zeta_{\vartheta}^{\perp}\right) p_{b} \cdot p_{b},
$$

and conclude as in Lemma 5.5, by symmetrizing the second term.

Theorem 2.9 finally follows from Lemmas 5.15 .2 and a rewriting of $E(q)$ and $B(q)$ taking (5.67) into account.

\section{Convergence to the massive point vortex system in Case (i): Proof of Theorem 2.11}

In this section we prove Theorem 2.11 which corresponds to Case (i). We will rely on intermediate results (Proposition 6.3, Lemma 6.4 and Lemma 6.5). The proofs of these intermediate results will be postponed to the last sections.

We work on Equation 2.21 with a small solid, that is

$$
M_{\varepsilon}\left(q_{\varepsilon}\right) q_{\varepsilon}^{\prime \prime}+\left\langle\Gamma_{\varepsilon}\left(q_{\varepsilon}\right), q_{\varepsilon}^{\prime}, q_{\varepsilon}^{\prime}\right\rangle=F_{\varepsilon}\left(q_{\varepsilon}, q_{\varepsilon}^{\prime}\right)
$$

where

$$
M_{\varepsilon}:=M\left[\mathcal{S}_{0, \varepsilon}, m_{\varepsilon}, \mathcal{J}_{\varepsilon}, \Omega\right], \Gamma_{\varepsilon}:=\Gamma\left[\mathcal{S}_{0, \varepsilon}, \Omega\right] \text { and } F_{\varepsilon}:=F\left[\mathcal{S}_{0, \varepsilon}, \gamma, \Omega\right],
$$


with $m_{\varepsilon}, \mathcal{J}_{\varepsilon}$ given by 2.38. The functions $M_{\varepsilon}(q),\left\langle\Gamma_{\varepsilon}(q), p, p\right\rangle$ and $F_{\varepsilon}(q, p)$ are defined for $q$ in $\mathcal{Q}^{\varepsilon}$ and for $p$ in $\mathbb{R}^{3}$.

We begin by introducing some notations. Given $\delta>0$ and $\varepsilon_{0}$ in $(0,1)$, we let

$$
\begin{gathered}
\mathfrak{Q}:=\left\{(\varepsilon, q) \in(0,1) \times \mathbb{R}^{3}: d\left(\mathcal{S}_{\varepsilon}(q), \partial \Omega\right)>0\right\}, \\
\mathfrak{Q}^{\delta}:=\left\{(\varepsilon, q) \in(0,1) \times \mathbb{R}^{3}: d\left(\mathcal{S}_{\varepsilon}(q), \partial \Omega\right)>\delta\right\}, \\
\mathfrak{Q}_{\delta, \varepsilon_{0}}:=\left\{(\varepsilon, q) \in\left(0, \varepsilon_{0}\right) \times \mathbb{R}^{3}: d\left(\mathcal{S}_{\varepsilon}(q), \partial \Omega\right)>\delta\right\} .
\end{gathered}
$$

For $\delta>0$, we also introduce

$$
\Omega_{\delta}:=\{x \in \Omega: d(x, \partial \Omega)>\delta\} .
$$

Observe that despite the fact that the center of mass $h_{\varepsilon}$ does not necessarily belong to $\mathcal{S}_{\varepsilon}(q)$, we have the following elementary result whose proof is left to the reader.

Lemma 6.1. Let $\delta>0$. There exists $\delta_{0}$ in $(0, \delta)$ and $\varepsilon_{0}$ in $(0,1]$ such that for any $(\varepsilon, q)$ in $\mathfrak{Q}_{\delta, \varepsilon_{0}}$, with $q=(\vartheta, h)$, necessarily $h$ belongs to $\Omega_{\delta_{0}}$.

\subsection{Normal form}

We will rephrase (6.1) to be able to pass to the limit as $\varepsilon$ goes to 0 . The following definition will be useful to deal with the remainder.

Definition 6.2. Let $\delta>0$ and $\varepsilon_{0} \in(0,1)$ be given. We say that a vector field $F$ in $L_{\text {loc }}^{\infty}\left(\mathfrak{Q}_{\delta, \varepsilon_{0}} \times \mathbb{R}^{3} ; \mathbb{R}^{3}\right)$ is weakly nonlinear if there exists $K>0$ depending on $\mathcal{S}_{0}, m, \mathcal{J}, \gamma, \Omega$ and $\delta$ such that for any $(\varepsilon, q, p)$ in $\mathfrak{Q}_{\delta, \varepsilon_{0}} \times \mathbb{R}^{3}$,

$$
|F(\varepsilon, q, p)|_{\mathbb{R}^{3}} \leqslant K\left(1+|p|_{\mathbb{R}^{3}}+\varepsilon|p|_{\mathbb{R}^{3}}^{2}\right) .
$$

The normal form is as follows.

Proposition 6.3. There exists $F_{r}: \mathfrak{Q} \times \mathbb{R}^{3} \rightarrow \mathbb{R}^{3}$ depending on $\mathcal{S}_{0}, \gamma$ and $\Omega$ such that, for any $\delta>0$, there exists $\varepsilon_{0}$ in $(0,1)$ such that $F_{r}$ belongs to $L_{\mathrm{loc}}^{\infty}\left(\mathfrak{Q}_{\delta, \varepsilon_{0}} \times \mathbb{R}^{3} ; \mathbb{R}^{3}\right)$ and is weakly nonlinear in the sense of Definition 6.2 and such that Equation (6.1) can be recast as

$$
M_{g} p_{\varepsilon}^{\prime}=F_{\vartheta_{\varepsilon}}^{\mathcal{S}_{0}}\left(\varepsilon \vartheta_{\varepsilon}^{\prime}, h_{\varepsilon}^{\prime}-\gamma u^{\Omega}\left(h_{\varepsilon}\right)\right)+\varepsilon F_{r}\left(\varepsilon, q_{\varepsilon}, p_{\varepsilon}\right) .
$$

Recall that $u^{\Omega}$ was defined in 2.44 , that the force term $F_{\vartheta_{\varepsilon}}^{\mathcal{S}_{0}}(p)$ was defined in the section dealing with the case without outer boundary, see (4.14), and that $p_{\varepsilon}=\left(\varepsilon \vartheta_{\varepsilon}^{\prime}, h_{\varepsilon}^{\prime}\right)^{t}$ was defined in 4.29 .

The normal form (6.7) will be useful in order to pass to the limit. To get Proposition 6.3. we will perform expansions of the inertia matrix, of the Christoffel symbols and of the force terms with respect to $\varepsilon$. Roughly speaking the leading terms coming from the force terms will be gathered into the first term of the right hand side of (6.7); see (9.60). 
6.2 Renormalized energy estimates

We will of course need uniform estimates as $\varepsilon \rightarrow 0^{+}$in order to pass to the limit in (6.7). The energy is the natural candidate to yield such estimates. Hence we are led to consider the behavior of the energy with respect to $\varepsilon$. We therefore now index the energy (introduced in in 2.22 ) as follows:

$$
\mathcal{E}_{\varepsilon}(q, p):=\frac{1}{2} M_{\varepsilon}(q) p \cdot p+U_{\varepsilon}(q),
$$

where the potential energy $U_{\varepsilon}$ is given by

$$
U_{\varepsilon}(q):=-\frac{1}{2} \gamma^{2} C_{\varepsilon}(q) .
$$

Of course Proposition 2.6 can be applied for each $\varepsilon$ in $(0,1)$ so that the energy associated with a solution $q_{\varepsilon}$ as in Theorem 2.11 is conserved along time until its maximal time of existence $T_{\varepsilon}$. We will establish in Subsection 8.3 the following result regarding the expansion of $C_{\varepsilon}(q)$ with respect to $\varepsilon$. The expansion is uniform, in the sense that the remainder is uniformly bounded, as long as the solid stays at a positive distance from the external boundary. Let us recall that the Newtonian potential $G$ was introduced in $(2.42$, the Kirchhoff-Routh stream function $\psi^{\Omega}$ was defined in 2.43 and the constant $C^{\mathcal{S}_{0}}$ in 4.5). We will also use the function defined for $q:=(\vartheta, h)$ in $\mathbb{R} \times \Omega$,

$$
\psi_{c}(q):=D_{h} \psi^{\Omega}(h) \cdot \zeta_{\vartheta} .
$$

Above $D_{h}$ denotes the derivative with respect to $h$.

Lemma 6.4. There exists a function $C_{r}: \mathfrak{Q} \rightarrow \mathbb{R}$ such that, for any $\delta>0$, there exists $\varepsilon_{0}$ in $(0,1)$ such that $C_{r}$ is in $L^{\infty}\left(\mathfrak{Q}_{\delta, \varepsilon_{0}} ; \mathbb{R}\right)$ and that for any $(\varepsilon, q)$ in $\mathfrak{Q}_{\delta, \varepsilon_{0}}$,

$$
C_{\varepsilon}(q)=-G(\varepsilon)+C^{\mathcal{S}_{0}}+2 \psi^{\Omega}(h)+2 \varepsilon \psi_{c}(q)+\varepsilon^{2} C_{r}(\varepsilon, q) .
$$

This result establishes that the potential energy $U_{\varepsilon}(q)$ diverges logarithmically as $\varepsilon \rightarrow 0^{+}$. However since they do not depend on the solid position and velocity the contributions of the two first terms of $(6.11)$ can be discarded from the energy in 6.8 without altering its conservation property.

On the other hand an immediate consequence of Proposition 9.1 below is the following result regarding the kinetic energy part.

Lemma 6.5. There is a function $M_{r}: \mathfrak{Q} \rightarrow \mathbb{R}^{3 \times 3}$ depending on $\mathcal{S}_{0}$ and $\Omega$, such that, for any $\delta>0$, there exists $\varepsilon_{0}$ in $(0,1)$ such that $M_{r}$ is in $L^{\infty}\left(\mathfrak{Q}_{\delta, \varepsilon_{0}} ; \mathbb{R}^{3 \times 3}\right)$ and such that for all $(\varepsilon, q)$ in $\mathfrak{Q}$, for all $p$ in $\mathbb{R}^{3}$,

$$
\left.\frac{1}{2} M_{\varepsilon}(q) p \cdot p=\frac{1}{2}\left(\left(M_{g}+\varepsilon^{2} M_{a, \vartheta}^{\mathcal{S}_{0}}\right) I_{\varepsilon} p\right)\right) \cdot\left(I_{\varepsilon} p\right)+\frac{1}{2} \varepsilon^{4}\left(M_{r}(\varepsilon, q) I_{\varepsilon} p\right) \cdot\left(I_{\varepsilon} p\right) .
$$

Recall that $M_{a, \vartheta}^{\mathcal{S}_{0}}$ was defined in 4.10 and $I_{\varepsilon}$ in 4.28 . Combining Lemma 6.4 and Lemma 6.5 we obtain the following. 
Corollary 6.6. Let $q_{\varepsilon}$ and $T_{\varepsilon}$ be as in Theorem 2.11. Then the renormalized energy

$$
\begin{aligned}
\frac{1}{2}\left(\left(M_{g}+\varepsilon^{2} M_{a, \vartheta}^{\mathcal{S}_{0}}\right) p_{\varepsilon}\right) & \cdot p_{\varepsilon}-\gamma^{2} \psi^{\Omega}\left(h_{\varepsilon}\right) \\
& +\frac{1}{2} \varepsilon^{4} M_{r}\left(\varepsilon, q_{\varepsilon}\right) p_{\varepsilon} \cdot p_{\varepsilon}-\varepsilon \gamma^{2} \psi_{c}\left(q_{\varepsilon}\right)-\frac{1}{2} \varepsilon^{2} \gamma^{2} C_{r}\left(\varepsilon, q_{\varepsilon}\right),
\end{aligned}
$$

is constant in time until $T_{\varepsilon}$.

The two most important terms in the renormalized energy above are the first and second ones which are respectively of order $O\left(\left|p_{\varepsilon}\right|_{\mathbb{R}^{3}}^{2}\right)$ and $O(1)$ as long as there is no collision. Hence we deduce the following counterpart of Corollary 2.7.

Corollary 6.7. Let $\left(q_{\varepsilon}, T_{\varepsilon}\right)$ as in Theorem 2.11. Let $\delta>0$. There exists $K>0$ (depending on $\mathcal{S}_{0}, \Omega, p_{0}, \gamma, m_{1}, \mathcal{J}_{1}, \delta$ ) and $\varepsilon_{0}>0$ such that for any $\varepsilon$ in $\left(0, \varepsilon_{0}\right)$, as long as $\left(\varepsilon, q_{\varepsilon}\right)$ belongs to $\mathfrak{Q}_{\delta, \varepsilon_{0}}$, one has $\left|p_{\varepsilon}\right|_{\mathbb{R}^{3}} \leqslant K$.

6.3 Passage to the limit

We deduce from Corollary 6.7 two different results. The first result concerns the lifetime $T_{\varepsilon}$ of the solution $\left(q_{\varepsilon}, p_{\varepsilon}\right)$, which can be only limited by a possible encounter between the solid and the boundary $\partial \Omega$.

Lemma 6.8. There exist $\varepsilon_{0}>0, \underline{T}>0$ and $\underline{\delta}>0$, such that for any $\varepsilon$ in $\left(0, \varepsilon_{0}\right)$,

$$
T^{\varepsilon} \geqslant \underline{T} \text { and on }[0, \underline{T}],\left(\varepsilon, q_{\varepsilon}\right) \in \mathfrak{Q}_{\underline{\delta}, \varepsilon_{0}} .
$$

Proof Let us introduce

$$
R_{0}:=\max \left\{|x|, x \in \partial \mathcal{S}_{0}\right\},
$$

so that, whatever $t \geqslant 0, \vartheta(t)$ in $\mathbb{R}$ and $\varepsilon$ in $(0,1)$,

$$
\mathcal{S}_{\varepsilon}\left(q_{\varepsilon}(t)\right) \subset \bar{B}\left(h_{\varepsilon}(t), \varepsilon R_{0}\right) .
$$

We introduce $\underline{\delta}:=\frac{1}{4} d(0, \partial \Omega)$ and $\varepsilon_{0}$ in $(0,1)$ (which may be reduced later) such that $\varepsilon_{0} R_{0} \leqslant \underline{\delta}$ and

$$
\forall \varepsilon \in\left(0, \varepsilon_{0}\right], \quad d\left(\bar{B}\left(0, \varepsilon R_{0}\right), \partial \Omega\right) \geqslant \frac{3}{4} d(0, \partial \Omega) .
$$

We apply Corollary 6.7 with $\underline{\delta}$ to deduce that there exists $K>0$ such that, reducing $\varepsilon_{0}$ if necessary,

$$
\left|h_{\varepsilon}^{\prime}\right| \leqslant K, \text { for all } t \text { for which } d\left(\mathcal{S}_{\varepsilon}\left(q_{\varepsilon}(t)\right), \partial \Omega\right) \geqslant \underline{\delta} .
$$

We introduce $\underline{T}:=\min \left(1, \frac{d(0, \partial \Omega)}{2 K}\right)$, and for $\varepsilon$ in $\left(0, \varepsilon_{0}\right]$,

$$
\mathcal{I}^{\varepsilon}=\left\{t \in[0,1]: \forall s \in[0, t], d\left(\bar{B}\left(h_{\varepsilon}(s), \varepsilon R_{0}\right), \partial \Omega\right) \geqslant \underline{\delta}\right\} .
$$


The set $\mathcal{I}^{\varepsilon}$ is a closed interval containing 0 , according to 6.15 . Consider $\tilde{T}_{\varepsilon}:=$ $\max \mathcal{I}^{\varepsilon}$, and let us show that $\tilde{T}_{\varepsilon} \geqslant \underline{T}$. Of course, if $\tilde{T}_{\varepsilon}=1$, then this is clear; let us suppose that $\tilde{T}_{\varepsilon}<1$. This involves that $d\left(\bar{B}\left(h_{\varepsilon}\left(\tilde{T}_{\varepsilon}\right), \varepsilon R_{0}\right), \partial \Omega\right)=\underline{\delta}$. Using $\varepsilon_{0} R_{0} \leqslant \underline{\delta}$ we deduce $d\left(h_{\varepsilon}\left(\tilde{T}_{\varepsilon}\right), \partial \Omega\right) \leqslant 2 \underline{\delta}$. With the triangle inequality and $\underline{\delta}=\frac{1}{4} d(0, \partial \Omega)$ we infer that $d\left(h_{\varepsilon}\left(\tilde{T}_{\varepsilon}\right), 0\right) \geqslant \frac{1}{2} d(0, \partial \Omega)$. Now the relation (6.14) implies that for all $t$ in $\left[0, \tilde{T}_{\varepsilon}\right]$,

$$
d\left(\mathcal{S}_{\varepsilon}\left(q_{\varepsilon}(t)\right), \partial \Omega\right) \geqslant d\left(\bar{B}\left(h_{\varepsilon}(t), \varepsilon R_{0}\right), \partial \Omega\right) \geqslant \underline{\delta},
$$

so that 6.16 is satisfied during $\left[0, \tilde{T}_{\varepsilon}\right]$. We deduce that $K \tilde{T}_{\varepsilon} \geqslant d(0, \partial \Omega) / 2$, so $\tilde{T}_{\varepsilon} \geqslant \underline{T}$. Therefore for any $t$ in $[0, \underline{T}]$, for any $\varepsilon$ in $\left[0, \varepsilon_{0}\right],(6.17)$ holds true. This concludes the proof of Lemma 6.8 .

The second result establishes the desired convergence on any time interval during which we have a minimal distance between $\mathcal{S}_{\varepsilon}(q)$ and $\partial \Omega$, uniform for small $\varepsilon$. Let us recall that $(\bar{h}, \bar{T})$ denotes the maximal solution to 2.40 .

Lemma 6.9. Let $\varepsilon_{1}>0, \check{\delta}>0$ and $\check{T}>0$ with $\check{T}<\bar{T}$, and suppose that for any $\varepsilon$ in $\left(0, \varepsilon_{1}\right)$,

$$
d\left(\mathcal{S}_{\varepsilon}\left(q_{\varepsilon}(t)\right), \partial \Omega\right) \geqslant \check{\delta} \text { on }[0, \check{T}] .
$$

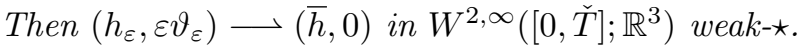

The proof of Lemma 6.9 consists in passing to the weak limit, with the help of all a priori bounds, in each term of (6.7). It is a straightforward extension of the proof of Theorem 4.9 and it is therefore omitted.

We now finish the proof of Theorem 2.11. It only remains to extend the time interval on which the above convergences are valid to any closed subinterval of $[0, \bar{T})$. Hence let $T \in(0, \bar{T})$, and let us prove that for small $\varepsilon>0$ the time of existence $T^{\varepsilon}$ is larger than $T$ and establish the convergences on the time interval $[0, T]$. For such a $T$, we know that there exists $\bar{d}>0$ such that

$$
\forall t \in\left[0, \frac{T+\bar{T}}{2}\right], \quad d(\bar{h}(t), \partial \Omega) \geqslant \bar{d} .
$$

We let $\bar{T}_{\varepsilon}:=\max \left\{t>0 d\left(B\left(h_{\varepsilon}(t), \varepsilon R_{0}\right), \partial \Omega\right) \geqslant \bar{d} / 2\right\}$. Let us recall that $R_{0}$ is defined in 6.13. Using Lemma 6.8 we deduce that, reducing $\bar{d}$ if necessary, for some $\bar{\varepsilon}>0, \inf _{\varepsilon \in(0, \bar{\varepsilon}]} \bar{T}_{\varepsilon}>0$. Therefore $\tilde{T}:=\liminf _{\varepsilon \rightarrow 0^{+}} \bar{T}_{\varepsilon}$ satisfies $\tilde{T}>0$. Due to Corollary 6.7, there exists $K>0$ and $\varepsilon_{0}$ such that for all $t$ in $[0, T+1]$ and $\varepsilon$ in $\left(0, \varepsilon_{0}\right)$,

$$
\left|h_{\varepsilon}^{\prime}\right|+\left|\varepsilon \vartheta_{\varepsilon}^{\prime}\right| \leqslant K \text { as long as } d\left(\mathcal{S}_{\varepsilon}\left(q_{\varepsilon}(t)\right), \partial \Omega\right) \geqslant \bar{d} / 2
$$

Now we claim that

$$
\tilde{T} \geqslant T+\frac{\bar{T}-T}{4}
$$

Suppose that this is not the case, so that there is a sequence $\varepsilon_{n} \rightarrow 0^{+}$such that $\bar{T}_{\varepsilon_{n}} \rightarrow \tilde{T}<T+\frac{\bar{T}-T}{4}$. Now for any $\eta$ in $(0, \tilde{T})$, on the interval $[0, \tilde{T}-\eta]$, 
the condition $d\left(\bar{B}\left(h_{\varepsilon_{n}}(t), \varepsilon R_{0}\right), \partial \Omega\right) \geqslant \bar{d} / 2$ is satisfied for $n$ large enough. Moreover, for such $n$, for all $t$ in $[0, \tilde{T}-\eta], 6.14$ implies that

$$
d\left(\mathcal{S}_{\varepsilon_{n}}\left(q_{\varepsilon_{n}}(t)\right), \partial \Omega\right) \geqslant d\left(\bar{B}\left(h_{\varepsilon_{n}}(t), \varepsilon_{n} R_{0}\right), \partial \Omega\right) \geqslant \bar{d} / 2 .
$$

Hence applying Lemma 6.9 , we deduce the uniform convergence of $\left(h_{\varepsilon_{n}}\right)$ to $\bar{h}$ on $[0, \tilde{T}-\eta]$. In particular, as $n \rightarrow+\infty$,

$$
d\left(h_{\varepsilon_{n}}(\tilde{T}-\eta), \partial \Omega\right) \rightarrow d(\bar{h}(\tilde{T}-\eta), \partial \Omega) \geqslant \bar{d},
$$

according to 6.19). On the other hand by definition of $\bar{T}_{\varepsilon_{n}}$

$$
d\left(B\left(h_{\varepsilon_{n}}\left(\bar{T}_{\varepsilon_{n}}\right), \varepsilon_{n} R_{0}\right), \partial \Omega\right)=\bar{d} / 2
$$

Using the triangle inequality and $\bar{T}_{\varepsilon_{n}} \rightarrow \tilde{T}$, we get a contradiction with 6.20 for $\eta$ small enough. Hence (6.21) is valid, so that, reducing $\bar{\varepsilon}$ if necessary, $\inf _{\varepsilon \in(0, \bar{\varepsilon}]} \bar{T}_{\varepsilon} \geqslant T$. Now, applying again (6.14) and Lemma 6.9, we reach the conclusion. This ends the proof of Theorem 2.11

\section{Convergence to the point vortex system in Case (ii): Proof of Theorem 2.12}

In this section we prove Theorem 2.12 which corresponds to Case (ii). We work again on Equations (6.1) but here $m_{\varepsilon}$, and $\mathcal{J}_{\varepsilon}$ are given by (2.46). Our analysis relies on some normal forms (see Propositions 7.10 and 7.18 below) which proved in subsequent sections. We begin this section with the case where $\mathcal{S}_{0}$ is a homogeneous disk because in this situation it is simpler to deduce the convergence from the normal form. Then we treat the case when $\mathcal{S}_{0}$ is not a disk, and finish with the case of a non-homogeneous disk.

7.1 The case of a homogeneous disk

When $\mathcal{S}_{0}$ is a homogeneous disk, the dynamics of the angle $\vartheta_{\varepsilon}$ is trivial; we expand the dynamics of the center of mass $h_{\varepsilon}$ in powers of $\varepsilon$.

Without loss of generality, we assume that $\mathcal{S}_{0}=\bar{B}(0,1)$. We first modify a bit Definition 6.2 as follows.

Definition 7.1. Let $\delta>0$ and $\varepsilon_{0} \in(0,1)$ be given. We say that a vector field $F_{\mathrm{b}}$ in $L_{\text {loc }}^{\infty}\left(\left(0, \varepsilon_{0}\right) \times \Omega \times \mathbb{R}^{2} ; \mathbb{R}^{2}\right)$ is weakly nonlinear if there exists $K>0$ depending on $m, \mathcal{J}, \gamma, \Omega$ and $\delta$ such that for any $\varepsilon$ in $\left(0, \varepsilon_{0}\right)$, any $h$ in $\Omega$ such that $d(B(h, \varepsilon), \partial \Omega)>\delta$ and any $\ell \in \mathbb{R}^{2}$,

$$
\left|F_{b}(\varepsilon, h, \ell)\right|_{\mathbb{R}^{2}} \leqslant K\left(1+|\ell|_{\mathbb{R}^{2}}+\varepsilon|\ell|_{\mathbb{R}^{2}}^{2}\right) .
$$

We used the notation 2.6. In this simpler situation we obtain the following result. 
Proposition 7.2. There exists $F_{b, r}$ in $L_{\mathrm{loc}}^{\infty}\left((0,1) \times \Omega \times \mathbb{R}^{2} ; \mathbb{R}^{2}\right)$ such that, for any $\delta>0$, there exists $\varepsilon_{0}$ in $(0,1)$ for which $F_{b, r}$ is weakly nonlinear in the sense of Definition 7.1 and such that Equation 2.35) can be recast as

$$
\vartheta_{\varepsilon}^{\prime \prime}=0,
$$

and

$$
\begin{aligned}
\left(\varepsilon^{\alpha} m_{1}+\varepsilon^{2} \pi\right)\left(h_{\varepsilon}^{\prime}-u^{\Omega}\left(h_{\varepsilon}\right)\right)^{\prime}=\gamma\left(h_{\varepsilon}^{\prime}-u^{\Omega}\left(h_{\varepsilon}\right)\right)^{\perp} & \\
& +\varepsilon^{\min (2, \alpha)} F_{b, r}\left(\varepsilon, h_{\varepsilon}, h_{\varepsilon}^{\prime}\right) .
\end{aligned}
$$

Since this proposition is a particular case of Proposition 7.10 (or of Proposition 7.18), we make no specific proof.

Note that the coefficient $\pi$ comes from the fact that $M_{b}^{\mathcal{S}_{0}}=\pi \mathrm{Id}_{2}$ in this case (where $M_{b}^{\mathcal{S}_{0}}$ is defined in 4.25). As a consequence, Corollary 6.7 has the following counterpart.

Corollary 7.3. Let $h_{\varepsilon}$ satisfy the assumptions of Theorem 2.9. Let $\delta>0$. There exists $K>0$ (depending on $\Omega, \ell_{0}, \gamma, m_{1}, \delta$ ) and $\varepsilon_{0}>0$ such that for any $\varepsilon$ in $\left(0, \varepsilon_{0}\right)$, for any $t>0$, as long as $d\left(B\left(h_{\varepsilon}, \varepsilon\right), \partial \Omega\right)>\delta$ one has $\varepsilon^{\min \left(1, \frac{\alpha}{2}\right)}\left|h_{\varepsilon}^{\prime}\right|_{\mathbb{R}^{2}} \leqslant K$.

Proof The proof is almost the same as for Corollary 6.7, but we have to take into account that the added mass matrix is degenerate and that dynamics of the rotation angle is trivial.

In the case of a homogeneous disk,the energy given in (6.8) can be described by using the following function, where $q=(\vartheta, h)$ and $p=(\omega, \ell)$ :

$$
\tilde{\mathcal{E}}_{\varepsilon}(h, \omega, \ell)=\frac{1}{2}\left(\varepsilon^{\alpha} m_{1} \operatorname{Id}_{2}+\varepsilon^{2} \tilde{M}_{b}(h)\right) \ell \cdot \ell+\varepsilon^{\alpha+2} \mathcal{J}_{1} \omega^{2}+\tilde{U}_{\varepsilon}(h) .
$$

Here we wrote $\tilde{U}_{\varepsilon}(h):=U_{\varepsilon}(q)$ for $q=(\vartheta, h)$ since actually it does not depend on the angle $\vartheta$.

Now using the same analysis as for Corollary 6.6 and taking into account that $\vartheta_{\varepsilon}^{\prime}(t)=\omega_{0}$ for all times, we deduce that the following quantity is conserved over time:

$$
\begin{aligned}
\frac{1}{2}\left(\varepsilon^{\alpha} m_{1} \operatorname{Id}_{2}+\varepsilon^{2} \tilde{M}_{b}(h)\right) h_{\varepsilon}^{\prime} \cdot h_{\varepsilon}^{\prime}-\gamma^{2} \psi^{\Omega}\left(h_{\varepsilon}\right) & \\
& +\frac{1}{2} \varepsilon^{4} \tilde{M}_{b, r}\left(\varepsilon, h_{\varepsilon}\right) h_{\varepsilon}^{\prime} \cdot h_{\varepsilon}^{\prime}+\varepsilon \tilde{C}_{r}\left(\varepsilon, h_{\varepsilon}\right),
\end{aligned}
$$

where $\tilde{C}_{r}\left(\varepsilon, h_{\varepsilon}\right):=C_{r}\left(\varepsilon, q_{\varepsilon}\right)$ since it does not depend on the angle, and $\tilde{M}_{b, r}$ is a bounded function on sets for which $d\left(B\left(h_{\varepsilon}, \varepsilon\right), \partial \Omega\right)>\delta$. The conclusion follows.

To improve the estimates on $h_{\varepsilon}^{\prime}$, we obtain modulated energy estimates. The following lemma relies on straightforward computations. 
Lemma 7.4. Let $h_{\varepsilon}$ satisfy the assumptions of Theorem 2.9. Then, during their lifetime, regular solutions satisfy

$$
\frac{d}{d t} \frac{1}{2}\left(\varepsilon^{2} \pi+\varepsilon^{\alpha} m_{1}\right)\left|h_{\varepsilon}^{\prime}-u^{\Omega}\left(h_{\varepsilon}\right)\right|^{2}=\varepsilon^{\min (2, \alpha)}\left(h_{\varepsilon}^{\prime}-u^{\Omega}\left(h_{\varepsilon}\right)\right) \cdot F_{b, r}\left(\varepsilon, h_{\varepsilon}, h_{\varepsilon}^{\prime}\right) .
$$

Moreover we have the following immediate estimate.

Lemma 7.5. Let $\delta>0$, there exists $\varepsilon_{0}$ in $(0,1)$ and $K>0$ such that for any $\varepsilon \in\left(0, \varepsilon_{0}\right)$, any $h \in \Omega$ such that $d(B(h, \varepsilon), \partial \Omega)>\delta$, we have $\left|u^{\Omega}(h)\right|_{\mathbb{R}^{2}} \leqslant K$.

We conclude that Corollary 7.3 can be improved into the following.

Corollary 7.6. Let $h_{\varepsilon}$ satisfy the assumptions of Theorem 2.9. Let $\delta>0$. There exists $K>0$ (depending on $\mathcal{S}_{0}, \Omega, \ell_{0}, \gamma, m_{1}, \delta$ ) and $\varepsilon_{0}>0$ such that for any $\varepsilon$ in $\left(0, \varepsilon_{0}\right)$, for any $t>0$ such that $d\left(B\left(h_{\varepsilon}(t), \varepsilon\right), \partial \Omega\right)>\delta$ one has $\left|h_{\varepsilon}^{\prime}(t)\right|_{\mathbb{R}^{2}} \leqslant K$.

Thanks to Corollary 7.6. Lemma 6.8 remains true in the case under view, that it, the time of existence $T_{\varepsilon}$ of $h_{\varepsilon}$ is bounded from below by some $\underline{T}>0$. Now we have the following local convergence result of $h_{\varepsilon}$ toward $\bar{h}$, where we recall that $\bar{h}$ is the global solution to 2.48 .

Lemma 7.7. Let $\varepsilon_{1}>0, \delta>0$ and $T>0$, and suppose that for any $\varepsilon$ in $\left(0, \varepsilon_{1}\right)$,

$$
d\left(B\left(h_{\varepsilon}(t), \varepsilon\right), \partial \Omega\right)>\delta \text { on }[0, T] .
$$

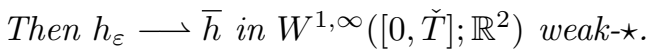

Proof Given $\delta>0, T>0$ and $\varepsilon_{1}>0$ as above we apply Corollary 7.6 on $[0, T]$ so that reducing $\varepsilon_{1}>0$ if necessary,

$$
\left(h_{\varepsilon}^{\prime}\right)_{\varepsilon \in\left(0, \varepsilon_{1}\right)} \text { is bounded uniformly on }[0, T] .
$$

Our goal is to pass to the limit in each term of 7.2 . For what concerns the left hand side it is obvious that $\left(\varepsilon^{\alpha} m_{1}+\varepsilon^{2} \pi\right)\left(h_{\varepsilon}^{\prime}-u^{\Omega}\left(h_{\varepsilon}\right)\right)^{\prime} \longrightarrow 0$ in $W^{-1, \infty}$. Next, the term $\varepsilon^{\min (2, \alpha)} F_{\mathrm{b}, r}\left(\varepsilon, h_{\varepsilon}, h_{\varepsilon}^{\prime}\right)$ converges to 0 in $L^{\infty}$. Hence we infer that $h_{\varepsilon}^{\prime}-\gamma u^{\Omega}\left(h_{\varepsilon}\right)$ converges weakly to 0 in $W^{-1, \infty}$. Due to the a priori estimate, this convergences occurs in $L^{\infty}$ weak- $\star$. By $(7.6)$, there is a subsequence $\left(h_{\varepsilon_{n}}\right)_{n}$ of $\left(h_{\varepsilon}\right)$ satisfying $h_{\varepsilon_{n}} \rightarrow h_{*}$ in $W^{1, \infty}$ weak- . In particular convergence of $h_{\varepsilon}$ toward $h_{*}$ is strong in $L^{\infty}$, and with the convergence of $h_{\varepsilon}^{\prime}-\gamma u^{\Omega}\left(h_{\varepsilon}\right)$ we deduce that $h_{*}^{\prime}=\gamma u^{\Omega}\left(h_{*}\right)$ and $h_{*}(0)=0$. The uniqueness of the solution to this Cauchy problem gives $h_{*}=\bar{h}$ and that the whole sequence $\left(h_{\varepsilon}\right)$ converges toward $\bar{h}$ as $\varepsilon \rightarrow 0^{+}$. This concludes the proof of Lemma 7.7 .

Finally we briefly conclude the proof of Theorem 2.12 in the case of a homogeneous disk, which is the same as the one of Theorem 2.11. except that in the case (ii) under view, as mentioned below 2.48), the solution $\bar{h}$ is global in time, and in particular that there is no collision of the point vortex with 
the external boundary $\partial \Omega$. Hence here, compared to the end of the proof of Theorem 2.11 at the end of Subsection 6.3 we can pick any $T>0$, and then we define $\bar{d}$ so that for all $t \in[0, T+1], d(h(t), \partial \Omega) \geqslant \bar{d}$ rather than by $(6.19)$ and prove $\tilde{T} \geqslant T+\frac{1}{2}$ rather than 6.21 . Of course we rely on Corollary 7.6 rather than Corollary 6.7 and on Lemma 7.7 rather than Lemma 6.9. This ends the proof of Theorem 2.12 in the case of a homogeneous disk.

\subsection{Geodesic-gyroscopic normal form}

In Case (ii) we will establish that (6.1) can be put into a normal form whose structure looks like (4.17) up to a refined modulation of the velocity of the center of mass. Indeed, in the same way as we defined the Kirchhoff-Routh velocity $u^{\Omega}$ by $u^{\Omega}=\nabla^{\perp} \psi^{\Omega}$ we introduce the corrector velocity $u_{c}$ corresponding to the stream function $\psi_{c}$ defined in 6.10 by

$$
u_{c}(q):=\nabla_{h}^{\perp} \psi_{c}(q) .
$$

Observe that the function $u_{c}$ depends on $\Omega, \mathcal{S}_{0}, \vartheta$ and on $h$, whereas $u^{\Omega}$ depends only on $\Omega$ and $h$. We will make use in a crucial way of the following second order modulation:

$$
\tilde{p}_{\varepsilon}:=\left(\varepsilon \vartheta_{\varepsilon}^{\prime}, h_{\varepsilon}^{\prime}-\gamma\left[u^{\Omega}\left(h_{\varepsilon}\right)+\varepsilon u_{c}\left(q_{\varepsilon}\right)\right]\right)^{t},
$$

which can be compared to the expression (4.29) of $p_{\varepsilon}$. We note that $\gamma\left(u^{\Omega}(h)+\right.$ $\left.\varepsilon u_{c}(q)\right)$ is the beginning of the expansion of $-\frac{1}{\gamma} \nabla_{h}^{\perp} U_{\varepsilon}(q)$ where $U_{\varepsilon}(q)$ is the electric-type potential energy defined in (6.9); see (6.11). This modulation is therefore driven by the leading terms of the electric-type potential. Observe also that, as long as the solid does not touch the boundary, the drift term in the velocity of the center of mass is bounded. Indeed the following refinement of Lemma 7.5 is a direct consequence of Lemma 6.1 and of the definitions of $u^{\Omega}$ and $u_{c}$.

Lemma 7.8. Let $\delta>0$, there exists $\varepsilon_{0}$ in $(0,1)$ and $K>0$ such that for any $(\varepsilon, q)$ in $\mathfrak{Q}_{\delta, \varepsilon_{0}}$ with $q=(\vartheta, h),\left|u^{\Omega}(h)+\varepsilon u_{c}(q)\right|_{\mathbb{R}^{3}} \leqslant K$.

Before stating our normal form, we introduce the following definition.

Definition 7.9. Let $\delta>0$ and $\varepsilon_{0} \in(0,1)$ be given. We say that a vector field $F$ in $C^{\infty}\left(\mathbb{R} \times \Omega ; \mathbb{R}^{3}\right)$ is weakly gyroscopic if there exists $K>0$ depending on $\mathcal{S}_{0}, \Omega, \gamma$ and $\delta$ such that for any smooth curve $q(t)=(\vartheta(t), h(t))$ in $\mathbb{R} \times \Omega_{\delta}$, for any $t \geqslant 0$ and any $\varepsilon \in\left(0, \varepsilon_{0}\right)$

$$
\left|\int_{0}^{t} \tilde{p} \cdot F(q)\right| \leqslant \varepsilon K\left(1+t+\int_{0}^{t}|\tilde{p}|_{\mathbb{R}^{3}}^{2}\right),
$$

with $\tilde{p}=\left(\varepsilon \vartheta^{\prime}, h^{\prime}-\gamma\left[u^{\Omega}(h)+\varepsilon u_{c}(q)\right]\right)^{t}$.

The weakly gyroscopic vector fields in the sequel have the form $F=$ $\left(F_{1}, 0,0\right)^{t}$. The normal form that we use in Case (ii) is as follows. 
Proposition 7.10. There exist

- $F_{r}: \mathfrak{Q} \times \mathbb{R}^{3} \rightarrow \mathbb{R}^{3}$ depending on $\mathcal{S}_{0}, \gamma$ and $\Omega$, such that for any $\delta>0$, there exists $\varepsilon_{0}$ in $(0,1)$ such that $F_{r} \in L_{\mathrm{loc}}^{\infty}\left(\mathfrak{Q}_{\delta, \varepsilon_{0}} \times \mathbb{R}^{3} ; \mathbb{R}^{3}\right)$ and is weakly nonlinear in the sense of Definition 6.2.

- $\mathrm{E}_{1}^{b}$ in $C^{\infty}\left(\mathbb{R} \times \Omega ; \mathbb{R}^{3}\right)$ depending on $\mathcal{S}_{0}$ and $\Omega$, weakly gyroscopic in the sense of Definition 7.9 .

such that Equation (6.1) can be recast as

$$
\begin{aligned}
\left(\varepsilon^{\alpha} M_{g}+\varepsilon^{2} M_{a, \vartheta_{\varepsilon}}^{\mathcal{S}_{0}}\right) \tilde{p}_{\varepsilon}^{\prime}+\varepsilon\left\langle\Gamma_{\vartheta_{\varepsilon}}^{\mathcal{S}_{0}}, \tilde{p}_{\varepsilon}, \tilde{p}_{\varepsilon}\right\rangle=F_{\vartheta_{\varepsilon}}^{\mathcal{S}_{0}}\left(\tilde{p}_{\varepsilon}\right)+\varepsilon \gamma^{2} \mathrm{E}_{1}^{b}\left(q_{\varepsilon}\right) \\
+\varepsilon^{\min (2, \alpha)} F_{r}\left(\varepsilon, q_{\varepsilon}, \tilde{p}_{\varepsilon}\right) .
\end{aligned}
$$

We recall that $M_{a, \vartheta}^{\mathcal{S}_{0}}, \Gamma_{\vartheta}^{\mathcal{S}_{0}}$ and the force term $F_{\vartheta}^{\mathcal{S}_{0}}$ were defined in the case without outer boundary in 4.10, 4.12 and 4.14 (see also 4.16) respectively. Moreover the term $\mathrm{E}_{1}^{b}$ is explicit; see (9.15). The proof of Proposition 7.10 is given in Section 9 .

- Motivations. We will use the normal form 7.10 both in order to get a uniform bound of the velocity and to pass to the limit in Case (ii). It would be actually possible to deal with the case where $\alpha$ is small with a less accurate normal form and still get an energy estimate.

In particular in order to get a uniform bound of the velocity in Case (ii) we will perform an estimate on an energy adapted to the normal form 7.10 . Observe that should the right hand side vanish the normal form 77.10 would be the geodesic equation associated with the metric $M_{\vartheta}(\varepsilon)$ defined in (4.33). On the other hand the right hand side is the sum of terms with a quite remarkable structure: the leading term $F_{\vartheta_{\varepsilon}}^{\mathcal{S}_{0}}\left(\tilde{p}_{\varepsilon}\right)$ is gyroscopic in the sense of Definition 4.3 , the electric-type term $\mathrm{E}_{1}^{b}\left(q_{\varepsilon}\right)$ is weakly gyroscopic in the sense of Definition 7.9 and the remainder $F_{r}$ is weakly nonlinear in the sense of Definition 6.2.

- Ideas of the proof of Proposition 7.10. As for Proposition 6.3 the proof of Proposition 7.10 relies on expansions of the inertia matrix, of the Christoffel symbols and of the force terms with respect to $\varepsilon$. A striking and crucial phenomenon is that some subprincipal contributions (that is, of order $\varepsilon$ ) of the force terms will be gathered with the leading part of the term involving the Christoffel symbols to become a part of the second term of the left hand side of 7.10); see Lemma 9.9. The leading part of the contribution coming from the Christoffel symbols will be provided by the $\Gamma^{\mathrm{rot}}$-part of the decomposition (2.28).

Remark 7.11. The normal forms above are inspired by the case without external boundary (see Equation (4.17) ) and by the paper [2] where the authors consider the motion of a light charged particle in a slowly varying electromagnetic field. The equation of motion for the particle is an ordinary differential equation involving a small parameter in front of the term with the highest time derivative. To restore some uniformity with respect to the small parameter 
they use a modulation, subtracting from the particle velocity the $|B|^{-2} E \times B$ drift, and a normal form, see [2, Eq. (3.5)], where the only remaining singular term appears through a Lorentz gyroscopic force. This allows us to tackle the convergence of the particle motion to the so-called guiding center motion despite the fast oscillations induced by the gyroscopic force.

In the case where the solid $\mathcal{S}_{0}$ is a homogeneous disk (Subsection 7.1), $u^{\Omega}\left(h_{\varepsilon}\right)$ is the vector whose coordinates are the last two coordinates of this drift. However in the case where $\mathcal{S}_{0}$ is not a disk our drift term $\left(0, \gamma\left(u^{\Omega}(h)+\varepsilon u_{c}(q)\right)\right)$ does not enter this framework. Actually the use of the $|B|^{-2} E \times B$ drift could give a modulated energy estimate only in the case $\alpha \leqslant 1$, and in particular not in the case of a solid with a fixed homogeneous density $(\alpha=2)$. Moreover it would not be adapted to the passage to the limit.

\subsection{Modulated energy estimates}

As mentioned above, in Case (i), Corollary 6.7 provides a uniform bound of $h_{\varepsilon}^{\prime}$ as long as the body stays at a positive distance from the external boundary. In Case (ii), the same analysis can be carried on so that Corollary 6.7 and Corollary 7.3 have the following counterpart.

Corollary 7.12. Let $q_{\varepsilon}$ satisfy the assumptions of Theorem 2.12. Let $\delta>0$. There exists $K>0$ (depending on $\mathcal{S}_{0}, \Omega, p_{0}, \gamma, m_{1}, \mathcal{J}_{1}, \delta$ ) and $\varepsilon_{0}>0$ such that for any $\varepsilon$ in $\left(0, \varepsilon_{0}\right)$, such that as long as $\left(\varepsilon, q_{\varepsilon}\right)$ belongs to $\mathfrak{Q}_{\delta, \varepsilon_{0}}$, one has $\varepsilon^{\min \left(1, \frac{\alpha}{2}\right)}\left|p_{\varepsilon}\right|_{\mathbb{R}^{3}} \leqslant K$.

Therefore this analysis does no longer provide a uniform bound of the solid velocity. An important part of the proof consists in finding an appropriate substitute which allows us a better control on the body velocity. This will be accomplished below by a modulated energy, which, roughly speaking, consists in applying the energy $\mathcal{E}_{\vartheta}$ (see 4.35p) to $\tilde{p}_{\varepsilon}$ (defined in (7.8p) rather than to $p_{\varepsilon}$ (defined in 4.29).

The structure established in Proposition 7.10 will allow us to obtain an estimate of the modulated energy $\mathcal{E}_{\vartheta}\left(\varepsilon, \tilde{p}_{\varepsilon}\right)$. Since Equation 7.10 looks like Equation (4.17) of the case without external boundary for which the total energy is the kinetic energy alone (as defined in Proposition 4.5), one may hope to have a good behaviour of the modulated energy $\mathcal{E}_{\vartheta}\left(\varepsilon, \widetilde{p}_{\varepsilon}\right)$ as time proceeds. Indeed we have the following result on the time derivative of the modulated energy.

Lemma 7.13. Let $\left(q_{\varepsilon}, T_{\varepsilon}\right)$ as in Theorem 2.12, Then

$$
\frac{d}{d t} \mathcal{E}_{\vartheta_{\varepsilon}}\left(\varepsilon, \tilde{p}_{\varepsilon}\right)=\varepsilon^{\max (1-\alpha,-1)} \gamma^{2} \tilde{p}_{\varepsilon} \cdot \mathrm{E}_{1}^{b}\left(q_{\varepsilon}\right)+\tilde{p}_{\varepsilon} \cdot F_{r}\left(\varepsilon, q, \tilde{p}_{\varepsilon}\right) .
$$

Proof Since the matrix $M_{\vartheta_{\varepsilon}}$ defined in 4.33 is symmetric,

$$
\frac{d}{d t} \mathcal{E}_{\vartheta_{\varepsilon}}\left(\varepsilon, \tilde{p}_{\varepsilon}\right)=\tilde{p}_{\varepsilon} \cdot M_{\vartheta_{\varepsilon}}(\varepsilon) \tilde{p}_{\varepsilon}^{\prime}+\frac{1}{2} \tilde{p}_{\varepsilon} \cdot\left(\frac{d}{d t} M_{\vartheta_{\varepsilon}}(\varepsilon)\right) \tilde{p}_{\varepsilon} .
$$


Recalling (4.34 and substituting 7.10 in 7.12 we arrive at

$$
\begin{aligned}
\varepsilon^{\min (2, \alpha)} \frac{d}{d t} \mathcal{E}_{\vartheta_{\varepsilon}}\left(\varepsilon, \tilde{p}_{\varepsilon}\right) & =\tilde{p}_{\varepsilon} \cdot F_{\vartheta_{\varepsilon}}^{\mathcal{S}_{0}}\left(\tilde{p}_{\varepsilon}\right) \\
& +\tilde{p}_{\varepsilon} \cdot\left(\frac{1}{2} \varepsilon^{\min (2, \alpha)}\left(\frac{d}{d t} M_{\vartheta_{\varepsilon}}(\varepsilon)\right) \tilde{p}_{\varepsilon}-\varepsilon\left\langle\Gamma_{\vartheta_{\varepsilon}}^{\mathcal{S}_{0}}, \tilde{p}_{\varepsilon}, \tilde{p}_{\varepsilon}\right\rangle\right) \\
& +\varepsilon \gamma^{2} \tilde{p}_{\varepsilon} \cdot \mathrm{E}_{1}^{b}\left(q_{\varepsilon}\right)+\varepsilon^{\min (2, \alpha)} \tilde{p}_{\varepsilon} \cdot F_{r}\left(\varepsilon, q_{\varepsilon}, \tilde{p}_{\varepsilon}\right) .
\end{aligned}
$$

Using that the force term is $(\vartheta, p) \mapsto F_{\vartheta}^{\mathcal{S}_{0}}(p)$ is gyroscopic in the sense of Definition 4.3 we deduce that the first term of the right hand side of the equation (7.13) vanishes. Moreover, going back to the definition of $M_{\vartheta}(\varepsilon)$,

$$
\varepsilon^{\min (2, \alpha)} \frac{d}{d t} M_{\vartheta_{\varepsilon}}(\varepsilon)=\varepsilon^{2} \frac{d}{d t} M_{a, \vartheta_{\varepsilon}}^{\mathcal{S}_{0}}=\varepsilon \frac{\partial M_{a, \vartheta}^{\mathcal{S}_{0}}}{\partial q}\left(\vartheta_{\varepsilon}\right) \cdot \tilde{p}_{\varepsilon},
$$

by taking advantage of the fact that the matrix $M_{a, \vartheta}^{\mathcal{S}_{0}}$ depends only on $\vartheta$ and not on the last two coordinates of $q$. Using (4.18) we conclude that the second term of the right hand side of Equation 7.13 vanishes. Therefore the equation 7.13 reduces to 7.11 and the proof of Lemma 7.13 is complete.

Now Corollary 6.7 and Lemma 7.8 already give us that $\varepsilon \tilde{p}_{\varepsilon}$ is bounded. Then we use that $F_{r}$ is weakly nonlinear in the sense of Definition 6.2, that $\mathrm{E}_{1}^{b}$ is weakly gyroscopic in the sense of Definition 7.9 (using Lemma 6.1), Lemma 4.8 and Gronwall's lemma to get the following result.

Corollary 7.14. Let $\left(q_{\varepsilon}, T_{\varepsilon}\right)$ as in Theorem 2.11. Let $\delta>0$. There exists $K>0$ (depending on $\mathcal{S}_{0}, \Omega, p_{0}, \gamma, m_{1}, \mathcal{J}_{1}, \delta$ ) and $\varepsilon_{0}>0$ such that for any $\varepsilon$ in $\left(0, \varepsilon_{0}\right)$, as long as $\left(\varepsilon, q_{\varepsilon}\right)$ belongs to $\mathfrak{Q}_{\delta, \varepsilon_{0}}$, one has $\left|p_{\varepsilon}\right|_{\mathbb{R}^{3}} \leqslant K$.

Corollary 7.14 therefore provides the same estimates for Case (ii) as Corollary 6.7 for Case (i).

Remark 7.15. A modulated energy argument was also used in 31 for a related issue but we would like to emphasize that the modulation occurs for a different part of the energy. More precisely, in the paper 31] an Euler-Vlasov system is introduced as a mean-field model for massless point particles moving in a perfect incompressible fluid. This system couples the incompressible Euler equation with a Vlasov equation which describes the dynamics of the massive particles, seen as a dispersed phase. This Vlasov equation is therefore the counterpart of the ordinary differential equation (2.40) of Case (i). The last section of [31] deals with the limit where the individual mass of the particles converges to 0 . Then a modulated energy is used in order to obtain a hydrodynamic convergence, that is the convergence of the macroscopic mixture velocity associated by the Biot-Savart law with both fluid vorticity and particles circulations. This modulated energy is inspired by the paper [4 of Brenier regarding the gyrokinetic limit for the Vlasov-Poisson system. Referring to the decomposition of the energy in 2.22 and to Eq. (78) in 31 we 
sketch the following opposition. In [31] the potential part of the energy or more exactly of its modulation is crucial. On the contrary we use here a modulation of the kinetic part of the energy in order to deduce the limit of the particle dynamics, the fluid dynamics being subjugated to the particle one.

\subsection{Passage to the limit}

Here we prove the convergence of the center of mass $h_{\varepsilon}$ to $\bar{h}$, where $\bar{h}$ is the global solution to 2.48.

First, thanks to Corollary 7.14, Lemma 6.8 remains true in Case (ii). Moreover we have the following counterpart of Lemma 6.9 and Lemma 7.7

Lemma 7.16. Let $\varepsilon_{1}>0, \delta>0$ and $T>0$, and suppose that for any $\varepsilon$ in $\left(0, \varepsilon_{1}\right)$

$$
\left(\varepsilon, q_{\varepsilon}\right) \in \mathfrak{Q}_{\delta, \varepsilon_{1}} \text { on }[0, T] .
$$

Then $h_{\varepsilon} \longrightarrow \bar{h}$ in $W^{1, \infty}\left([0, T] ; \mathbb{R}^{2}\right)$ weak- .

As for Lemma 7.7. the proof of Lemma 7.16 consists in passing to the weak limit, with the help of all a priori bounds, in each term of (7.10).

Proof We consider $\delta>0, T>0$ and $\varepsilon_{1}>0$ as above and apply Corollary 7.14 on the interval $[0, T]$. Reducing $\varepsilon_{1}>0$ if necessary,

$$
\left(\left|h_{\varepsilon}^{\prime}\right|+\left|\varepsilon \vartheta_{\varepsilon}^{\prime}\right|\right)_{\varepsilon \in\left(0, \varepsilon_{1}\right)} \text { is bounded uniformly on }[0, T] .
$$

Our goal is to pass to the limit in each term of 7.10 . For what concerns the left hand side we first observe that, thanks to (7.16), $\varepsilon M_{a, \mathcal{S}_{0}, \vartheta_{\varepsilon}}$ is bounded in $W^{1, \infty}$ whereas $\tilde{p}_{\varepsilon}^{\prime}$ is bounded in $W^{-1, \infty}$. Since $M_{g}$ is constant, it follows that $\left(\varepsilon^{\alpha} M_{g}+\varepsilon^{2} M_{a, \vartheta_{\varepsilon}}^{\mathcal{S}_{0}}\right) \tilde{p}_{\varepsilon}^{\prime} \longrightarrow 0$ in $W^{-1, \infty}$. Next, the term $\varepsilon\left\langle\Gamma_{\vartheta_{\varepsilon}}^{\mathcal{S}_{0}}, \tilde{p}_{\varepsilon}, \tilde{p}_{\varepsilon}\right\rangle$ converges to 0 in $L^{\infty}$ since all factors in the brackets are bounded. In the same way, the terms $\varepsilon \gamma^{2} \mathrm{E}_{1}^{b}\left(q_{\varepsilon}\right)$ and $\varepsilon^{\min (2, \alpha)} F_{r}\left(\varepsilon, q_{\varepsilon}, \tilde{p}_{\varepsilon}\right)$ converge strongly to 0 in $L^{\infty}$.

Now let us consider the last two coordinates of the remaining term $F_{\vartheta_{\varepsilon}}^{\mathcal{S}_{0}}\left(\tilde{p}_{\varepsilon}\right)$ in the equation 7.10 , that is, $\gamma\left(h_{\varepsilon}^{\prime}-\gamma u^{\Omega}\left(h_{\varepsilon}\right)-\varepsilon \gamma u_{c}\left(q_{\varepsilon}\right)\right)^{\perp}-\varepsilon \vartheta_{\varepsilon}^{\prime} \zeta_{\vartheta_{\varepsilon}}$. The last term converges weakly to 0 in $W^{-1, \infty}$ as seen in Case (i); see 4.38 . Hence we infer that $h_{\varepsilon}^{\prime}-\gamma u^{\Omega}\left(h_{\varepsilon}\right)$ converges weakly to 0 in $W^{-1, \infty}$. Then one concludes exactly as for Lemma 7.7 .

Once Lemma 7.16 is established, the conclusion of the proof of Theorem 2.12 (provided that $\mathcal{S}_{0}$ is not a disk) follows the exact same lines as in the case of a homogeneous disk. This concludes the proof of Theorem 2.12 in this case.

\subsection{The case of a non-homogeneous disk}

We now return to the case $\mathcal{S}_{0}$ is a disk, but this time we handle the case where it is non-homogeneous, or more precisely the case where the center of 
gravity $h_{\varepsilon}$ is not at the center of the disk $h_{c, \varepsilon}$, which can occur with a nonuniform mass distribution. We aim at proving Theorem 2.13. This adds some extra-difficulties in the analysis which require a separate treatment in the case $\alpha>2$. In the case $\alpha \leqslant 2$, we can use the above subsection since the degeneracy of $M_{a, \vartheta}^{\mathcal{S}_{0}}$ does not prevent $M_{\vartheta}(\varepsilon)$ to be uniformly bounded from below, so that we can obtain Corollary 7.14 in the same way.

From now on we suppose $\alpha>2$ and once again we assume without loss of generality $\mathcal{S}_{0}=\bar{B}(0,1)$. We will use yet another slight variant of Definition 6.2 and Definition 7.1

Definition 7.17. Let $\delta>0$ and $\varepsilon_{0} \in(0,1)$ be given. We say that a vector field $F_{\mathrm{b}}$ in $L_{\text {loc }}^{\infty}\left(\left(0, \varepsilon_{0}\right) \times \Omega \times \mathbb{R}^{3} ; \mathbb{R}^{2}\right)$ is weakly nonlinear if there exists $K>0$ depending on $m, \mathcal{J}, \gamma, \Omega$ and $\delta$ such that for any $\varepsilon$ in $\left(0, \varepsilon_{0}\right)$, any $x$ in $\Omega$ such that $d(B(x, \varepsilon), \partial \Omega)>\delta$ and any $p$ in $\mathbb{R}^{3}$,

$$
\left|F_{b}(\varepsilon, x, p)\right|_{\mathbb{R}^{2}} \leqslant K\left(1+|p|_{\mathbb{R}^{3}}+\varepsilon|p|_{\mathbb{R}^{3}}^{2}\right) .
$$

We will use the modulated variable $\tilde{p}_{b, \varepsilon}$ defined by

$$
\tilde{p}_{b, \varepsilon}:=h_{c, \varepsilon}^{\prime}-\gamma u^{\Omega}\left(h_{c, \varepsilon}\right)
$$

and the following normal form.

Proposition 7.18. There exists $F_{\mathrm{b}, r}$ in $L_{\mathrm{loc}}^{\infty}\left((0,1) \times \Omega \times \mathbb{R}^{3} ; \mathbb{R}^{2}\right)$ such that, for any $\delta>0$, there exists $\varepsilon_{0}$ in $(0,1)$ for which $F_{b, r}$ is weakly nonlinear in the sense of Definition 7.17, and such that Equation (2.35) can be recast as

$$
\begin{aligned}
\varepsilon^{\alpha} m_{1} h_{\varepsilon}^{\prime \prime}+\varepsilon^{2} \pi\left(\tilde{p}_{b, \varepsilon}\right)^{\prime} & =\gamma \tilde{p}_{b, \varepsilon}^{\perp}+\varepsilon^{2} F_{b, r}\left(\varepsilon, h_{c, \varepsilon}, \varepsilon \vartheta_{\varepsilon}^{\prime}, \tilde{p}_{b, \varepsilon}\right), \\
h_{c, \varepsilon}-h_{\varepsilon} & =\varepsilon \zeta_{\vartheta_{\varepsilon}}, \\
\mathcal{J}_{1} \varepsilon \vartheta_{\varepsilon}^{\prime \prime} & =\zeta_{\vartheta_{\varepsilon}}^{\perp} \cdot m_{1} h_{\varepsilon}^{\prime \prime}
\end{aligned}
$$

Again the coefficient $\pi$ comes from the fact that $M_{b}^{\mathcal{S}_{0}}=\pi \operatorname{Id}_{2}$ (where $M_{b}^{\mathcal{S}_{0}}$ is defined in (4.25). The proof of Proposition 7.18 is given in Section 9

Once Proposition 7.18 is obtained, for $\varepsilon \in\left(0, \varepsilon_{0}\right)$, we multiply 7.18$)$ by $\tilde{p}_{b, \varepsilon}$. We set $\mathcal{E}_{b}:=\varepsilon^{\alpha} m_{1}\left|h_{\varepsilon}^{\prime}\right|^{2}+\varepsilon^{\alpha+2} \mathcal{J}_{1}\left|\vartheta_{\varepsilon}^{\prime}\right|^{2}+\varepsilon^{2} \pi\left|\tilde{p}_{b, \varepsilon}\right|^{2}$. Noticing that

$$
m_{1} h_{\varepsilon}^{\prime \prime} \cdot h_{c, \varepsilon}^{\prime}=m_{1} h_{\varepsilon}^{\prime \prime} \cdot h_{\varepsilon}^{\prime}+\varepsilon^{2} \mathcal{J}_{1} \vartheta_{\varepsilon}^{\prime \prime} \vartheta_{\varepsilon}^{\prime}
$$

we arrive at

$$
\frac{d}{d t} \mathcal{E}_{b}(t)+\varepsilon^{\alpha} m_{1} h_{\varepsilon}^{\prime \prime} \cdot u^{\Omega}\left(h_{c, \varepsilon}\right) \leqslant C \varepsilon^{2}\left|\tilde{p}_{b, \varepsilon}\right|\left(1+\left|\tilde{p}_{b, \varepsilon}\right|+\varepsilon\left|\tilde{p}_{b, \varepsilon}\right|^{2}\right)
$$

The standard energy estimate gives us here that $\varepsilon\left|h_{c, \varepsilon}^{\prime}\right|$ is bounded, and consequently so is $\varepsilon\left|\tilde{p}_{b, \varepsilon}\right|$. Integrating over time and using an integration by parts we obtain (as long as the solid stays at positive distance from $\partial \Omega$ ):

$$
\mathcal{E}_{b}(t)-\mathcal{E}_{b}(0) \leqslant C \varepsilon^{2}+C \int_{0}^{t} \mathcal{E}_{b}(s) d s+C \varepsilon^{\alpha}\left|h_{\varepsilon}^{\prime}(t)\right|+C \varepsilon^{\alpha}\left|h_{\varepsilon}^{\prime}(0)\right| .
$$


Thanks to Young's inequality,

$$
\mathcal{E}_{b}(t) \leqslant C \int_{0}^{t} \mathcal{E}_{b}(s) d s+C \mathcal{E}_{b}(0)+C \varepsilon^{2},
$$

so with Gronwall's lemma we finally deduce that $\mathcal{E}_{b}(t) \leqslant C\left(\mathcal{E}_{b}(0)+\varepsilon^{2}\right)$, as long as the solid stays at positive distance from $\partial \Omega$. Since $\alpha>2$, we deduce that $\left(h_{c, \varepsilon}^{\prime}\right)_{\varepsilon \in\left(0, \varepsilon_{0}\right)},\left(\varepsilon^{\frac{\alpha}{2}-1} h_{\varepsilon}^{\prime}\right)_{\varepsilon \in\left(0, \varepsilon_{0}\right)}$ and $\left(\varepsilon^{\frac{\alpha}{2}} \vartheta_{\varepsilon}^{\prime}\right)_{\varepsilon \in\left(0, \varepsilon_{0}\right)}$ are bounded in $L^{\infty}$. Notice in passing that using (7.19) and an interpolation argument we deduce that $\left(h_{\varepsilon}\right)_{\varepsilon \in\left(0, \varepsilon_{0}\right)}$ is bounded in $W^{\frac{2}{\alpha}}, \infty$; see the discussion after the statement of Theorem 2.13

Then it is straightforward to adapt the previous reasoning to pass to the weak limit in 7.18 . We deduce $\tilde{p}_{b, \varepsilon} \rightarrow 0$ in $L^{\infty}$ and the conclusion follows. This ends the proof of Theorem 2.13 .

\section{Asymptotic development of the stream and potential functions}

In this section, we establish asymptotic expansions for the circulation stream function (defined in (2.11) ) and the Kirchhoff potentials (defined in (2.9p) in the domain $\mathcal{F}^{\varepsilon}(q)$, as $\varepsilon$ tends to $0^{+}$. The asymptotic analysis of the Laplace equation when the size of an inclusion goes to 0 has been deeply studied, see for example [20] and 29]. However to our knowledge the results of this section are not covered by the literature.

\subsection{A few reminders about single-layer potentials}

To get the asymptotic expansions mentioned above, we will look for a representation of these stream and potential functions as a superposition of singlelayer integrals supported by the two connected components $\partial \mathcal{S}^{\varepsilon}(q)$ and $\partial \Omega$ of the boundary of the fluid domain $\mathcal{F}_{\varepsilon}(q)$. In this subsection, we give a few reminders about single-layer potentials which we will use in the analysis. We refer for instance to [7] and [28.

Below we consider single-layer potentials of the form:

$$
S L\left[\mathrm{p}^{\mathcal{C}}\right]:=\int_{\mathcal{C}} \mathrm{p}^{\mathcal{C}}(y) G(\cdot-y) \mathrm{d} s(y),
$$

where $\mathcal{C}$ is a smooth Jordan curve in the plane and $\mathrm{p}^{\mathcal{C}}$ belongs to the Sobolev space $H^{-\frac{1}{2}}(\mathcal{C})$. Recall that $G$ was defined in 2.42 . We say that $\mathcal{C}$ is the support of the single-layer potential and that $\mathrm{p}^{\mathcal{C}}$ is a density on $\mathcal{C}$.

Harmonicity and trace. The formula (8.1) defines a function in the Sobolev space $H_{\text {loc }}^{1}\left(\mathbb{R}^{2}\right)$, harmonic in $\mathbb{R}^{2} \backslash \mathcal{C}$. In particular, for any $\mathrm{p}^{\mathcal{C}}$ in $H^{-\frac{1}{2}}(\mathcal{C})$, the trace of $S L\left[\mathrm{p}^{\mathcal{C}}\right]$ on $\mathcal{C}$ is well-defined as a function of the Sobolev space $H^{\frac{1}{2}}(\mathcal{C})$. 
Jump of the derivative and density. The density $\mathrm{p}^{\mathcal{C}}$ is equal to the jump of the normal derivative of $S L\left[\mathrm{p}^{\mathcal{C}}\right]$ across $\mathcal{C}$. To state this rigorously let us be specific on the orientation of the normal. According to Jordan's theorem, the set $\mathbb{R}^{2} \backslash \mathcal{C}$ has two connected components, one bounded (the interior), say $\mathcal{O}_{i}$, and the other one unbounded (the exterior), say $\mathcal{O}_{e}$. Moreover the curve $\mathcal{C}$ is the boundary of each component. We consider the restrictions of $S L\left[\mathrm{p}^{\mathcal{C}}\right]$ :

$$
u_{i}:=S L\left[\mathrm{p}^{\mathcal{C}}\right]_{\mid \mathcal{O}_{i}} \text { and } u_{e}:=S L\left[\mathrm{p}^{\mathcal{C}}\right]_{\mid \mathcal{O}_{e}}
$$

Denote $n_{i}$ (respectively $n_{e}$ ) the unit normal on $\mathcal{C}$ pointing outward of $\mathcal{O}_{i}$ (resp. of $\mathcal{O}_{e}$ ). Then the function $u_{i}$ (respectively $u_{e}$ ) is harmonic in $\mathcal{O}_{i}\left(\operatorname{resp} . \mathcal{O}_{e}\right.$ ) and the traces of the normal derivatives $\frac{\partial u_{i}}{\partial n_{i}}$ and $\frac{\partial u_{e}}{\partial n_{e}}$ on each side of $\mathcal{C}$ are well-defined in $H^{-\frac{1}{2}}(\mathcal{C})$ and satisfy

$$
\mathrm{p}^{\mathcal{C}}=\frac{\partial u_{i}}{\partial n_{i}}+\frac{\partial u_{e}}{\partial n_{e}}
$$

In the sequel we will make use of single-layer potentials supported on the external boundary $\partial \Omega$, on the boundary $\partial \mathcal{S}_{\varepsilon}(q)$ of the solid body and on the boundary $\partial \mathcal{S}_{0}$ of the rescaled body as well. We will not use the notations $n_{i}$ nor $n_{e}$ but rather the notation $n$ which always stands for the normal outward the fluid. Hence we will have to particularly take care of the signs when referring to the formula 8.2 .

Kernel and rank. We will use the following facts (see Th. 7.17, Th. 8.12 and Th. 8.16 in [28]):

The operator $S L$ is Fredholm with index zero from $L^{2}(\mathcal{C})$ to $H^{1}(\mathcal{C})$,

$$
\begin{aligned}
& \text { If } \mathrm{p}^{\mathcal{C}} \in H^{-\frac{1}{2}}(\mathcal{C}) \text { satisfies } \int_{\mathcal{C}} \mathrm{p}^{\mathcal{C}} \mathrm{d} s=0 \text { and } S L\left[\mathrm{p}^{\mathcal{C}}\right]=0 \text { then } \mathrm{p}^{\mathcal{C}}=0, \\
& \text { If } \operatorname{Cap}(\mathcal{C}) \neq 1 \text {, then for any } \mathrm{p}^{\mathcal{C}} \in H^{-\frac{1}{2}}(\mathcal{C}), S L\left[\mathrm{p}^{\mathcal{C}}\right]=0 \text { implies } \mathrm{p}^{\mathcal{C}}=0 \text {. }
\end{aligned}
$$

Above, with some slight abuses of notation, we omit to mention the trace operator on $\mathcal{C}$ and we write $\int_{\mathcal{C}} \mathrm{p}^{\mathcal{C}} \mathrm{d} s$ for the duality bracket $\left\langle 1, \mathrm{p}^{\mathcal{C}}\right\rangle_{H^{-\frac{1}{2}}(\mathcal{C}), H^{\frac{1}{2}}(\mathcal{C})}$.

These properties have the following consequences in our context. We assume without loss of generality that the logarithmic capacity ${ }^{2} \operatorname{Cap}(\partial \Omega)$ of $\partial \Omega$ satisfies $\operatorname{Cap}(\partial \Omega)<1$, using translation and dilatation of the coordinates system if necessary. Observe that the monotonicity property of the logarithmic capacity entails that $\operatorname{Cap}\left(\partial \mathcal{S}_{0}\right)<1$. Using this latter property, we deduce the two following results.

Proposition 8.1. There exists a unique smooth function $\psi_{-1}^{\mathcal{S}_{0}}$ solution to 4.5. Moreover, it satisfies

$$
\psi_{-1}^{\mathcal{S}_{0}}=S L\left[\mathrm{p}_{-1}^{\mathcal{S}_{0}}\right], \text { with } \mathrm{p}_{-1}^{\mathcal{S}_{0}}=\frac{\partial \psi_{-1}^{\mathcal{S}_{0}}}{\partial n} .
$$

2 also called external conformal radius or transfinite diameter in other contexts 37. 
In potential theory $-\mathrm{p}_{-1}^{\mathcal{S}_{0}}$ is called the equilibrium density of $\partial \mathcal{S}_{0}$. The constant $C^{\mathcal{S}_{0}}$ in 4.5 is given by $C^{\mathcal{S}_{0}}=\frac{1}{2 \pi} \ln \left(\operatorname{Cap}\left(\partial \mathcal{S}_{0}\right)\right)$. Note that the index -1 is in italic type to emphasize the fact that it is related to an asymptotic development of $\psi$ in powers of $\varepsilon$ and is not a coordinate.

Proposition 8.2. Let $g$ be a smooth function on $\partial \mathcal{S}_{0}$ such that

$$
\int_{\partial \mathcal{S}_{0}} g \mathrm{p}_{-1}^{\mathcal{S}_{0}} \mathrm{~d} s=0
$$

Then there exists a unique bounded smooth function $f$ such that

$$
-\Delta f=0 \quad \text { in } \quad \mathbb{R}^{2} \backslash \mathcal{S}_{0}, \quad \text { and } \quad f=g \quad \text { on } \partial \mathcal{S}_{0} .
$$

Moreover, there exists a unique smooth density $p^{\partial \mathcal{S}_{0}}$ in $C^{\infty}\left(\partial \mathcal{S}_{0}\right)$ such that $f=S L\left[p^{\partial \mathcal{S}_{0}}\right]$ and

$$
\int_{\partial \mathcal{S}_{0}} p^{\partial \mathcal{S}_{0}} \mathrm{~d} s=0
$$

Finally, $f=O\left(|x|_{\mathbb{R}^{2}}^{-1}\right)$ at infinity and

$$
\int_{\partial \mathcal{S}_{0}} \frac{\partial f}{\partial n} \mathrm{~d} s=0
$$

Proof of Proposition 8.1 and 8.2. The uniqueness part of Proposition 8.1 and of Proposition 8.2 and the decay at infinity in Proposition 8.2 can be established by considering holomorphy at infinity of appropriate functions; see for instance [7, Prop. 2.74. and Prop. 3.2.].

The existence part of Proposition 8.1 is given in [28, Th. 8.15]; it also follows from the properties of the single-layers potentials recalled above, in particular 8.3 and 8.5.

Regarding the existence part of Proposition 8.2 we proceed in two steps.

First we prove that the operator which maps $\left(p^{\partial \mathcal{S}_{0}}, C\right)$ in $L^{2}\left(\partial \mathcal{S}_{0}\right) \times \mathbb{R}$ to $\left(S L\left[p^{\partial \mathcal{S}_{0}}\right]-C, \int_{\partial \mathcal{S}_{0}} p^{\partial \mathcal{S}_{0}} \mathrm{~d} s\right)$ in $H^{1}\left(\partial \mathcal{S}_{0}\right) \times \mathbb{R}$ is invertible. To prove this, we observe that this operator is Fredholm with index zero as a consequence of 8.3. Moreover if $\left(p^{\partial \mathcal{S}_{0}}, C\right)$ is in the kernel of this operator, then $S L\left[p^{\partial \mathcal{S}_{0}}-\right.$ $\left.\frac{C}{C^{\mathcal{S}_{0}}} \mathrm{p}_{-1}^{\mathcal{S}_{0}}\right]=0$, so that according to $8.5, p^{\partial \mathcal{S}_{0}}=\frac{C}{C^{\mathcal{S}_{0}}} \mathrm{p}_{-1}^{\mathcal{S}_{0}}$. Now, as a consequence of $4.5 \mathrm{~d}$ ) and of the second identity of 8.6 ,

$$
\int_{\partial \mathcal{S}_{0}} \mathrm{p}_{-1}^{\mathcal{S}_{0}} \mathrm{~d} s=-1 .
$$

Then using that $\int_{\partial \mathcal{S}_{0}} p^{\partial \mathcal{S}_{0}} \mathrm{~d} s=0$ we deduce that $C=0$ and therefore $p^{\partial \mathcal{S}_{0}}=0$ as well.

Then $(g, 0)$ is in the image of this operator, that is there exists $\left(p^{\partial \mathcal{S}_{0}}, C\right)$ in $L^{2}\left(\partial \mathcal{S}_{0}\right) \times \mathbb{R}$ such that

$$
S L\left[p^{\partial \mathcal{S}_{0}}\right]-C=g \text { on } \partial \mathcal{S}_{0},
$$


and 8.9 . Observing that the trace of the operator $S L$ on $\partial \mathcal{S}_{0}$ is self-adjoint we infer that

$$
\int_{\partial \mathcal{S}_{0}} S L\left[p^{\partial \mathcal{S}_{0}}\right] \mathrm{p}_{-1}^{\mathcal{S}_{0}} \mathrm{~d} s=\int_{\partial \mathcal{S}_{0}} p^{\partial \mathcal{S}_{0}} S L\left[\mathrm{p}_{-1}^{\mathcal{S}_{0}}\right] \mathrm{d} s=C^{\mathcal{S}_{0}} \int_{\partial \mathcal{S}_{0}} p^{\partial \mathcal{S}_{0}} \mathrm{~d} s=0 .
$$

Combining (8.7), 8.11), 8.12) and 8.13) we infer that $C=0$.

Finally the smoothness part of Proposition 8.1 and of Proposition 8.2 follows from [28, Th. 7.16] and (8.10) follows from (8.9), 8.2) and the vanishing by integration by parts of the interior contribution.

Regular integral operators. Since we consider single-layer potentials supported on two disjoint curves and their values on both curves, we will also be led to consider regular integral operators. We recall below some straightforward results which are useful in the sequel. Given $\mathcal{C}$ a smooth Jordan curve in $\bar{\Omega}$, we introduce, for $\delta>0$,

$$
\mathcal{C}^{\delta}:=\{x \in \bar{\Omega}: \operatorname{dist}(x, \mathcal{C})<\delta\}
$$

and define

$$
F_{\delta}: C^{1}\left(\bar{\Omega} \backslash \mathcal{C}^{\delta} ; H^{\frac{1}{2}}(\mathcal{C})\right) \times H^{-\frac{1}{2}}(\mathcal{C}) \rightarrow C^{1}\left(\bar{\Omega} \backslash \mathcal{C}^{\delta} ; \mathbb{R}\right),
$$

by setting, for any $\left(b, \mathrm{p}^{\mathcal{C}}\right)$ in $C^{1}\left(\bar{\Omega} \backslash \mathcal{C}^{\delta} ; H^{\frac{1}{2}}(\mathcal{C})\right) \times H^{-\frac{1}{2}}(\mathcal{C})$, for any $x$ in $\bar{\Omega} \backslash \mathcal{C}^{\delta}$,

$$
F_{\delta}\left[b, \mathrm{p}^{\mathcal{C}}\right](x):=\int_{\mathcal{C}} b(x, y) \mathrm{p}^{\mathcal{C}}(y) \mathrm{d} s(y)
$$

This will be applied to $b$ defined in a larger set but singular for $x=y$; this motivates our framework for $b$.

Next, given another smooth Jordan curve $\tilde{\mathcal{C}}$ in $\bar{\Omega} \backslash \mathcal{C}^{\delta}$ and for $b$ in $C^{1}(\bar{\Omega} \backslash$ $\left.\mathcal{C}^{\delta} ; H^{\frac{1}{2}}(\mathcal{C})\right)$, we define the operator

$$
F_{\delta, b}: L^{2}(\mathcal{C}) \rightarrow H^{1}(\tilde{\mathcal{C}})
$$

by setting $F_{\delta, b}\left(\mathrm{p}^{\mathcal{C}}\right)$ as the trace of $F_{\delta}\left[b, \mathrm{p}^{\mathcal{C}}\right]$ on $\tilde{\mathcal{C}}$. We will make use of the following lemma.

Lemma 8.3. Let $\delta>0$. The two following properties hold.

(i) The operator $F_{\delta}$ is bilinear continuous with a norm less than 1 , in other words: for any $\left(b, \mathrm{p}^{\mathcal{C}}\right)$ in $C^{1}\left(\bar{\Omega} \backslash \mathcal{C}^{\delta} ; H^{\frac{1}{2}}(\mathcal{C})\right) \times H^{-\frac{1}{2}}(\mathcal{C})$,

$$
\left\|F_{\delta}\left[b, \mathrm{p}^{\mathcal{C}}\right]\right\|_{C^{1}\left(\bar{\Omega} \backslash \mathcal{C}^{\delta}\right)} \leqslant\|b\|_{C^{1}\left(\bar{\Omega} \backslash \mathcal{C}^{\delta} ; H^{\frac{1}{2}}(\mathcal{C})\right.}\left\|\mathrm{p}^{\mathcal{C}}\right\|_{H^{-\frac{1}{2}}(\mathcal{C})} .
$$

(ii) If $\tilde{\mathcal{C}}$ is a smooth Jordan curve in $\bar{\Omega} \backslash \mathcal{C}^{\delta}$ and b in $C^{1}\left(\bar{\Omega} \backslash \mathcal{C}^{\delta} ; H^{\frac{1}{2}}(\mathcal{C})\right)$, the operator $F_{\delta, b}$ is compact from $L^{2}(\mathcal{C})$ to $H^{1}(\tilde{\mathcal{C}})$.

The proof of Lemma 8.3 is elementary and left to the reader. 
8.2 Statements of the results

\subsubsection{Circulation part}

Let $(\varepsilon, q)$ in $\mathfrak{Q}$. We denote $\psi_{\varepsilon}(q, \cdot)$ in $\mathcal{F}_{\varepsilon}(q)$ the function defined as the solution to the Dirichlet boundary value problem:

$$
\begin{aligned}
& -\Delta \psi_{\varepsilon}(q, \cdot)=0 \quad \text { in } \mathcal{F}_{\varepsilon}(q), \\
& \psi_{\varepsilon}(q, \cdot)=C_{\varepsilon}(q) \text { on } \partial \mathcal{S}_{\varepsilon}(q), \\
& \psi_{\varepsilon}(q, \cdot)=0 \quad \text { on } \partial \Omega \text {, }
\end{aligned}
$$

where the constant $C_{\varepsilon}(q)$ is such that:

$$
\int_{\partial \mathcal{S}_{\varepsilon}(q)} \frac{\partial \psi_{\varepsilon}}{\partial n}(q, \cdot) \mathrm{d} s=-1 .
$$

Here, $n$ stands for the unit normal vector to $\partial \mathcal{S}_{\varepsilon}(q) \cup \partial \Omega$ directed toward the exterior of $\mathcal{F}_{\varepsilon}(q)$. The function $\psi_{\varepsilon}$ is the counterpart, for the case where the size of the solid is of order $\varepsilon$, of the function $\psi$ defined in 2.11 in the case where the size of the solid is of order 1 . For any $q$ in $\mathfrak{Q}$, the existence and uniqueness of a solution $\psi_{\varepsilon}(q, \cdot)$ of 8.14 is classical.

To state a result establishing an asymptotic expansion of $C_{\varepsilon}(q)$ and of $\frac{\partial \psi_{\varepsilon}}{\partial n}(q, \cdot)$ on $\partial \mathcal{S}_{\varepsilon}(q)$ as $\varepsilon \rightarrow 0^{+}$we introduce a few notations.

Definition of $\psi_{0}^{\mathcal{S}_{0}}(q, \cdot)$ and of $P_{0}(q, X)$. We denote, for any $q:=(\vartheta, h)$ in $\mathbb{R} \times \Omega$, by $P_{0}(q, X)$ the harmonic polynomial

$$
P_{0}(q, X):=u^{\Omega}(h)^{\perp} \cdot\left(R(\vartheta) X-\zeta_{\vartheta}\right) .
$$

Let us recall that $\zeta_{\vartheta}$ is defined in 4.15 in terms of $\zeta$ defined in 4.13).

Recalling (8.11) and the second identity of 8.6 we observe that $P_{0}(q, X)$ satisfies

$$
\int_{\partial \mathcal{S}_{0}} P_{0}(q, \cdot) \mathrm{p}_{-1}^{\mathcal{S}_{0}} \mathrm{~d} s=0
$$

Therefore, according to Proposition 8.2 there exists a unique smooth function $\psi_{0}^{\mathcal{S}_{0}}(q, \cdot)$ satisfying

$$
\begin{aligned}
-\Delta \psi_{0}^{\mathcal{S}_{0}}(q, \cdot) & =0 & & \text { in } \mathbb{R}^{2} \backslash \mathcal{S}_{0}, \\
\psi_{0}^{\mathcal{S}_{0}}(q, \cdot) & =P_{0}(q, \cdot) & & \text { on } \partial \mathcal{S}_{0},
\end{aligned}
$$

and vanishing at infinity. Moreover

$$
\int_{\partial \mathcal{S}_{0}} \frac{\partial \psi_{0}^{\mathcal{S}_{0}}}{\partial n} \mathrm{~d} s=0 .
$$


Definition of $\psi_{1}^{\Omega}(q, \cdot)$. We also introduce the solution $\psi_{1}^{\Omega}(q, \cdot)$ of

$$
\begin{aligned}
-\Delta \psi_{1}^{\Omega}(q, \cdot) & =0 & & \text { in } \Omega, \\
\psi_{1}^{\Omega}(q, \cdot) & =-(\nabla G)(\cdot-h) \cdot \zeta_{\vartheta} & & \text { on } \partial \Omega .
\end{aligned}
$$

Above $G$ denotes the Newtonian potential defined in $(2.42)$. The function $\psi_{1}^{\Omega}$ can be expressed thanks to the function $\psi_{0}^{\Omega}$ defined in (2.41) according to the following formula:

$$
\forall h, x \in \Omega, \quad \forall \vartheta \in \mathbb{R}, \quad D_{x} \psi_{0}^{\Omega}(h, x) \cdot \zeta_{\vartheta}=\psi_{1}^{\Omega}(\vartheta, x, h) .
$$

Proof of 8.19 . We first recall that $\psi_{0}^{\Omega}$ is symmetric in its variables. Indeed by uniqueness of the Dirichlet problem 2.41 it follows that for any $h$ in $\Omega$, the following decomposition holds in $\Omega$ :

$$
\psi_{0}^{\Omega}(h, \cdot)=G(\cdot-h)+G^{\Omega}(h, \cdot),
$$

where $G^{\Omega}$ denotes the Green function associated with the domain $\Omega$ and the homogeneous Dirichlet condition, that is

$$
\Delta G^{\Omega}(h, \cdot)=\delta_{h} \text { in } \Omega, G^{\Omega}(h, \cdot)=0 \text { on } \partial \Omega .
$$

Using the decomposition 8.20 , that the Newtonian potential $G$ is even and the symmetry of $G^{\Omega}$ we deduce

$$
\forall h, x \in \Omega, \quad \psi_{0}^{\Omega}(h, x)=\psi_{0}^{\Omega}(x, h) .
$$

It follows that $\left(D_{x} \psi_{0}^{\Omega}\right)(x, h) \cdot \zeta_{\vartheta}=\left(D_{h} \psi_{0}^{\Omega}\right)(h, x) \cdot \zeta_{\vartheta}$. Next we observe that $D_{h} \psi_{0}^{\Omega}(h, \cdot) \cdot \zeta_{\vartheta}$ satisfies the same Dirichlet problem as $\psi_{1}^{\Omega}(\vartheta, h, \cdot)$, by derivation of 2.41). Formula (8.19) follows then from the uniqueness of solutions to the Dirichlet problem, after switching $h$ and $x$.

Definition of $\psi_{1}^{\mathcal{S}_{0}}(q, \cdot)$ and of $P_{1}(q, X)$. Let us denote, for any $q:=(\vartheta, h)$ in $\mathbb{R} \times \Omega$, by $P_{1}(q, X)$ the polynomial defined by

$$
\begin{aligned}
P_{1}(q, X):=-\frac{1}{2}\left\langle R(\vartheta)^{t} D_{x}^{2} \psi_{0}^{\Omega}(h, h) R(\vartheta), T^{2}\left(\mathrm{p}_{-1}^{\mathcal{S}_{0}}\right)+X^{\otimes 2}\right\rangle_{\mathbb{R}^{2 \times 2}} \\
+R(\vartheta)^{t} D_{x} \psi_{1}^{\Omega}(q, h) \cdot(\zeta-X)
\end{aligned}
$$

Above $D_{x}^{2} \psi_{0}^{\Omega}(h, h)$ denotes the second derivative of $\psi_{0}^{\Omega}(h, \cdot)$ evaluated in $h$, $D_{x} \psi_{1}^{\Omega}(q, h)$ stands for the derivative of $\psi_{1}^{\Omega}(q, \cdot)$ evaluated in $h$ and $X^{\otimes 2}$ stands for the $2 \times 2$ matrix $X \otimes X$. The notation $T^{2}\left(\mathrm{p}_{-1}^{\mathcal{S}_{0}}\right)$ stands for

$$
T^{2}\left(\mathrm{p}_{-1}^{\mathcal{S}_{0}}\right):=\int_{\partial \mathcal{S}_{0}} \frac{\partial \psi_{-1}^{\mathcal{S}_{0}}}{\partial n}(X) X^{\otimes 2} \mathrm{~d} s(X) .
$$


This notation is justified by (8.6). Observe that $P_{1}(q, X)$ is harmonic since every monomial of Taylor's expansions of harmonic functions are themselves harmonic. By (4.13), 4.15) and the second identity of 8.6),

$$
\int_{\partial \mathcal{S}_{0}} \mathrm{p}_{-1}^{\mathcal{S}_{0}}(\zeta-X) \mathrm{d} s=0 .
$$

Thus, by 8.11 and 8.24,

$$
\int_{\partial \mathcal{S}_{0}} P_{1}(q, \cdot) \mathrm{p}_{-1}^{\mathcal{S}_{0}} \mathrm{~d} s=0 .
$$

Therefore, according to Proposition 8.2 there exists a unique smooth function $\psi_{1}^{\mathcal{S}_{0}}(q, \cdot)$ satisfying

$$
\begin{aligned}
-\Delta \psi_{1}^{\mathcal{S}_{0}}(q, \cdot) & =0 & & \text { in } \mathbb{R}^{2} \backslash \mathcal{S}_{0}, \\
\psi_{1}^{\mathcal{S}_{0}}(q, \cdot) & =P_{1}(q, \cdot) & & \text { on } \partial \mathcal{S}_{0},
\end{aligned}
$$

and vanishing at infinity. Moreover

$$
\int_{\partial \mathcal{S}_{0}} \frac{\partial \psi_{1}^{\mathcal{S}_{0}}}{\partial n}(q, \cdot) \mathrm{d} s=0 .
$$

Our main result regarding the potential $\psi_{\varepsilon}$, in addition to Lemma 6.4 is the following.

Proposition 8.4. There exist $\mathrm{p}_{r}^{\partial \mathcal{S}_{0}}: \mathfrak{Q} \rightarrow L^{2}\left(\partial \mathcal{S}_{0} ; \mathbb{R}\right)$, depending only on $\mathcal{S}_{0}$ and $\Omega$, such that, for any $\delta>0$, there exists $\varepsilon_{0}$ in $(0,1)$ for which $\mathrm{p}_{r}^{\partial \mathcal{S}_{0}} \in$ $L^{\infty}\left(\mathfrak{Q}_{\delta, \varepsilon_{0}} ; L^{2}\left(\partial \mathcal{S}_{0} ; \mathbb{R}\right)\right)$, and such that for any $(\varepsilon, q)$ in $\mathfrak{Q}_{\delta, \varepsilon_{0}}$ and for any $X$ in $\partial \mathcal{S}_{0}$,

$$
\begin{aligned}
\frac{\partial \psi_{\varepsilon}}{\partial n}(q, \varepsilon R(\vartheta) X+h)= & \frac{1}{\varepsilon} \frac{\partial \psi_{-1}^{\mathcal{S}_{0}}}{\partial n}(X)+\left(\frac{\partial \psi_{0}^{\mathcal{S}_{0}}}{\partial n}(q, X)-R(\vartheta)^{t} u^{\Omega}(h) \cdot \tau\right) \\
& +\varepsilon\left(\frac{\partial \psi_{1}^{\mathcal{S}_{0}}}{\partial n}-\frac{\partial P_{1}}{\partial n}\right)(q, X)+\varepsilon^{2} \mathbf{p}_{r}^{\partial \mathcal{S}_{0}}(\varepsilon, q, X) .
\end{aligned}
$$

We recall that the set $\mathfrak{Q}_{\delta, \varepsilon_{0}}$ was defined in 6.4 .

The proofs of Lemma 6.4 and of Proposition 8.4 are gathered in Subsection 8.3

\subsubsection{Potential part}

For any $j=1,2,3$, for any $q$ in $\mathcal{Q}$, we consider the functions $K_{j, \varepsilon}(q, \cdot)$ on $\partial \Omega \cup \partial \mathcal{S}_{\varepsilon}(q)$ given by:

$$
K_{j, \varepsilon}(q, \cdot):=n \cdot \xi_{j}(q, \cdot) \text { on } \partial \Omega \cup \partial \mathcal{S}_{\varepsilon}(q),
$$


where $n$ denotes the unit normal to $\partial \mathcal{S}_{\varepsilon}(q) \cup \partial \Omega$, pointing outside $\mathcal{F}^{\varepsilon}(q)$ and the functions $\xi_{j}(q, \cdot)$ are given by the formula (2.7). Then the Kirchhoff potentials $\varphi_{j, \varepsilon}(q, \cdot)$, for $j=1,2,3$, are the unique (up to an additive constant) solutions in $\mathcal{F}^{\varepsilon}(q)$ of the following Neumann problem:

$$
\begin{array}{ll}
\Delta \varphi_{j, \varepsilon}(q, \cdot)=0 & \text { in } \mathcal{F}^{\varepsilon}(q), \\
\frac{\partial \varphi_{j, \varepsilon}}{\partial n}(q, \cdot)=K_{j, \varepsilon}(q, \cdot) & \text { on } \partial \mathcal{S}_{\varepsilon}(q), \\
\frac{\partial \varphi_{j, \varepsilon}}{\partial n}(q, \cdot)=0 & \text { on } \partial \Omega .
\end{array}
$$

The functions $K_{j, \varepsilon}(q, \cdot)$ (respectively $\varphi_{j, \varepsilon}(q, \cdot)$ ) are the counterpart, for the case where the size of the solid is of order $\varepsilon$, of the functions defined in (2.8) (resp. in (2.9p) in the case where the size of the solid is of order 1.

We will use the vector notations:

$$
\boldsymbol{\varphi}_{\varepsilon}=\left(\varphi_{1, \varepsilon}, \varphi_{2, \varepsilon}, \varphi_{3, \varepsilon}\right)^{t} \text { and } \boldsymbol{K}_{\varepsilon}=\left(K_{1, \varepsilon}, K_{2, \varepsilon}, K_{3, \varepsilon}\right)^{t} \text {. }
$$

Our result on the expansion of the Kirchhoff potentials $\varphi_{j, \varepsilon}$ is the following.

Proposition 8.5. There exist

(i) $\varphi_{r}: \mathfrak{Q} \rightarrow L^{2}\left(\partial \mathcal{S}_{0} ; \mathbb{R}^{3}\right)$ and $\check{\boldsymbol{c}}: \mathfrak{Q} \rightarrow \mathbb{R}^{3}$ such that for any $(\varepsilon, q)$ in $\mathfrak{Q}$, with $q=(\vartheta, h)$, for any $X$ in $\partial \mathcal{S}_{0}$,

$$
\boldsymbol{\varphi}_{\varepsilon}(q, \varepsilon R(\vartheta) X+h)=\varepsilon I_{\varepsilon} \mathcal{R}(\vartheta)\left(\varphi^{\mathcal{S}_{0}}(X)+\check{\boldsymbol{c}}(\varepsilon, q)+\varepsilon^{2} \boldsymbol{\varphi}_{r}(\varepsilon, q, X)\right)
$$

(ii) $\mathrm{p}_{r}^{\partial \mathcal{S}_{0}}: \mathfrak{Q} \rightarrow L^{2}\left(\partial \mathcal{S}_{0} ; \mathbb{R}^{3}\right)$ such that for any $(\varepsilon, q)$ in $\mathfrak{Q}$ with $q=(\vartheta, h)$, for any $X$ in $\partial \mathcal{S}_{0}$,

$$
\mathcal{R}(\vartheta)^{t} \frac{\partial \varphi_{\varepsilon}}{\partial \tau}(q, \varepsilon R(\vartheta) X+h)=I_{\varepsilon}\left(\frac{\partial \varphi^{\mathcal{S}_{0}}}{\partial \tau}(X)+\varepsilon^{2} \mathrm{p}_{r}^{\partial \mathcal{S}_{0}}(\varepsilon, q, X)\right)
$$

(iii) $\mathrm{p}_{r}^{\partial \Omega}: \mathfrak{Q} \rightarrow L^{2}\left(\partial \Omega ; \mathbb{R}^{3}\right)$ such that for any $(\varepsilon, q)$ in $\mathfrak{Q}$, for any $x$ in $\partial \Omega$,

$$
\frac{\partial \varphi_{\varepsilon}}{\partial \tau}(q, x)=I_{\varepsilon} \varepsilon^{2} \mathrm{p}_{r}^{\partial \Omega}(\varepsilon, q, x)
$$

and such that for any $\delta>0$, there exists $\varepsilon_{0}$ in $(0,1)$ such that $\boldsymbol{\varphi}_{r}$ belongs to $L^{\infty}\left(\mathfrak{Q}_{\delta, \varepsilon_{0}} ; L^{2}\left(\partial \mathcal{S}_{0} ; \mathbb{R}^{3}\right)\right)$, г to $L^{\infty}\left(\mathfrak{Q}_{\delta, \varepsilon_{0}} ; \mathbb{R}^{3}\right), \mathrm{p}_{r}^{\partial \mathcal{S}_{0}}$ to $L^{\infty}\left(\mathfrak{Q}_{\delta, \varepsilon_{0}} ; L^{2}\left(\partial \mathcal{S}_{0} ; \mathbb{R}^{3}\right)\right)$ and $\mathrm{p}_{r}^{\partial \Omega}$ to $L^{\infty}\left(\mathfrak{Q}_{\delta, \varepsilon_{0}} ; L^{2}\left(\partial \Omega ; \mathbb{R}^{3}\right)\right)$.

Moreover the remainders $\boldsymbol{\varphi}_{r}, \mathrm{p}_{r}^{\partial \mathcal{S}_{0}}$ and $\mathrm{p}_{r}^{\partial \Omega}$ depend only on $\mathcal{S}_{0}$ and $\Omega$.

We recall that $\varphi^{\mathcal{S}_{0}}$ was defined in 4.3 . The proof of Proposition 8.5 is given in Subsection 8.4 . 
8.3 Asymptotic expansion of the circulation part: Proof of Proposition 8.4 and of Lemma 6.4

In this subsection we prove Proposition 8.4 and Lemma 6.4. Let, for $-\frac{1}{2} \leqslant$ $s \leqslant 1, F_{s}$ denote the following Hilbert space:

$$
F_{s}:=H^{s}\left(\partial \mathcal{S}_{0}\right) \times H^{s}(\partial \Omega) \times \mathbb{R}
$$

We will mainly make use of the indices $s=0$ and 1 and also for technical reasons of $-\frac{1}{2}$ and $\frac{1}{2}$. We will proceed in four steps.

\subsubsection{First Step. Reduction to integral equations}

We look for the solution $\psi_{\varepsilon}(q, \cdot)$ of (8.14) as a superposition of two single-layer integrals, one supported on the body's boundary and the other one supported on $\partial \Omega$. This transforms 8.14 in an integral system as follows.

We define, for any $(\varepsilon, q)$ in $\mathfrak{Q}$ with $q=(\vartheta, h)$ in $\mathbb{R} \times \Omega$, two operators $K^{\partial \mathcal{S}_{0}}(\varepsilon, q)$ and $K^{\partial \Omega}(\varepsilon, q)$ respectively from $L^{2}(\partial \Omega)$ to $H^{1}\left(\partial \mathcal{S}_{0}\right)$ and from $L^{2}\left(\partial \mathcal{S}_{0}\right)$ to $H^{1}(\partial \Omega)$, by the following formulas: given densities $\mathrm{p}^{\partial \Omega}$ and $\mathrm{p}^{\partial \mathcal{S}_{0}}$ respectively in $L^{2}(\partial \Omega)$ and $L^{2}\left(\partial \mathcal{S}_{0}\right)$,

$$
\begin{gathered}
K^{\partial \mathcal{S}_{0}}(\varepsilon, q)\left[\mathrm{p}^{\partial \Omega}\right](\cdot):=S L\left[p^{\partial \Omega}\right](\varepsilon R(\vartheta) \cdot+h) \text { on } \partial \mathcal{S}_{0}, \\
K^{\partial \Omega}(\varepsilon, q)\left[\mathrm{p}^{\partial \mathcal{S}_{0}}\right](\cdot):=\int_{\partial \mathcal{S}_{0}} \mathrm{p}^{\partial \mathcal{S}_{0}}(Y) G(\cdot-(\varepsilon R(\vartheta) Y+h)) \mathrm{d} s(Y) \text { on } \partial \Omega .
\end{gathered}
$$

Thanks to Lemma 8.3 (ii), the operators $K^{\partial \mathcal{S}_{0}}(\varepsilon, q)$ and $K^{\partial \Omega}(\varepsilon, q)$ are compact respectively from $L^{2}(\partial \Omega)$ to $H^{1}\left(\partial \mathcal{S}_{0}\right)$ and from $L^{2}\left(\partial \mathcal{S}_{0}\right)$ to $H^{1}(\partial \Omega)$.

We also introduce for $(\varepsilon, q)$ in $\mathfrak{Q}$, the operator $\mathfrak{A}(\varepsilon, q): F_{0} \rightarrow F_{1}$ as follows: for any $\mathfrak{p}:=\left(\mathrm{p}^{\partial \mathcal{S}_{0}}, \mathrm{p}^{\partial \Omega}, C\right)$ in $F_{0}$,

$$
\begin{aligned}
\mathfrak{A}(\varepsilon, q)[\mathfrak{p}]:=\left(S L\left[\mathrm{p}^{\partial \mathcal{S}_{0}}\right]+\right. & K^{\partial \mathcal{S}_{0}}(\varepsilon, q)\left[\mathrm{p}^{\partial \Omega}\right]-C, \\
& \left.S L\left[\mathrm{p}^{\partial \Omega}\right]+K^{\partial \Omega}(\varepsilon, q)\left[\mathrm{p}^{\partial \mathcal{S}_{0}}\right], \int_{\partial \mathcal{S}_{0}} \mathrm{p}^{\partial \mathcal{S}_{0}} \mathrm{~d} s\right) .
\end{aligned}
$$

To simplify the notations, we omitted to write the trace operators applied to the single-layers in $K^{\partial \mathcal{S}_{0}}(\varepsilon, q), K^{\partial \Omega}(\varepsilon, q)$ and $\mathfrak{A}(\varepsilon, q)$. We also emphasize that the dependence of $\mathfrak{A}(\varepsilon, q)$ on $(\varepsilon, q)$ occurs only through the compact operators $K^{\partial \mathcal{S}_{0}}(\varepsilon, q)$ and $K^{\partial \Omega}(\varepsilon, q)$.

Now the equation (8.14) is transformed into an integral system thanks to the following lemma.

Lemma 8.6. For any $(\varepsilon, q)$ in $\mathfrak{Q}$, let $\mathfrak{p}_{\varepsilon}(q, \cdot)=\left(p_{\varepsilon}^{\partial \mathcal{S}_{0}}(q, \cdot), p_{\varepsilon}^{\partial \Omega}(q, \cdot), C_{\varepsilon}(q)\right)$ in $F_{0}$ such that

$$
\mathfrak{A}(\varepsilon, q)\left[\mathfrak{p}_{\varepsilon}(q, \cdot)+(0,0, G(\varepsilon))\right]=(0,0,-1) .
$$


Consider the density $p_{\varepsilon}^{\partial \mathcal{S}_{\varepsilon}(q)}(q, \cdot)$ on $\partial \mathcal{S}_{\varepsilon}(q)$ defined through the relation:

$$
\text { for } X \in \partial \mathcal{S}_{0}, \quad p_{\varepsilon}^{\partial \mathcal{S}_{0}}(q, X):=\varepsilon p_{\varepsilon}^{\partial \mathcal{S}_{\varepsilon}(q)}(q, \varepsilon R(\vartheta) X+h) .
$$

Then the function in $\mathcal{F}^{\varepsilon}(q)$

$$
\psi_{\varepsilon}(q, \cdot):=S L\left[p_{\varepsilon}^{\partial \mathcal{S}_{\varepsilon}(q)}(q, \cdot)\right]+S L\left[p_{\varepsilon}^{\partial \Omega}(q, \cdot)\right],
$$

is the solution to 8.14. Moreover the normal derivative $\frac{\partial \psi_{\varepsilon}}{\partial n}(q, \cdot)$ on $\partial \mathcal{S}_{\varepsilon}(q)$ is given by:

$$
\text { for } X \in \partial \mathcal{S}_{0}, \quad \frac{\partial \psi_{\varepsilon}}{\partial n}(q, \varepsilon R(\vartheta) X+h)=\frac{1}{\varepsilon} p_{\varepsilon}^{\partial \mathcal{S}_{0}}(q, X) .
$$

Proof First observe that for any densities $p_{\varepsilon}^{\partial \mathcal{S}_{\varepsilon}(q)}(q, \cdot)$ in $H^{-\frac{1}{2}}\left(\partial \mathcal{S}_{\varepsilon}(q)\right)$ and $p_{\varepsilon}^{\partial \Omega}(q, \cdot)$ in $H^{-\frac{1}{2}}(\partial \Omega)$, the right hand side of $(8.39)$ is in $H_{\text {loc }}^{1}\left(\mathbb{R}^{2}\right)$ and harmonic in $\mathcal{F}_{\varepsilon}(q)$ and in $\mathbb{R}^{2} \backslash \mathcal{F}_{\varepsilon}(q)$. In particular the equation 8.14a is satisfied when $\psi^{\varepsilon}(q, \cdot)$ is given by 8.39 without further assumptions about $p_{\varepsilon}^{\partial \mathcal{S}_{\varepsilon}(q)}(q, \cdot)$ or $p_{\varepsilon}^{\partial \Omega}(q, \cdot)$.

Next we write 8.37 explicitly in the form:

$$
\begin{aligned}
-G(\varepsilon)+S L\left[p_{\varepsilon}^{\partial \mathcal{S}_{0}}(q, \cdot)\right]+K^{\partial \mathcal{S}_{0}}(\varepsilon, q)\left[p_{\varepsilon}^{\partial \Omega}(q, \cdot)\right] & =C_{\varepsilon}(q) & & \text { on } \partial \mathcal{S}_{0}, \\
S L\left[p_{\varepsilon}^{\partial \Omega}(q, \cdot)\right]+K^{\partial \Omega}(\varepsilon, q)\left[p_{\varepsilon}^{\partial \mathcal{S}_{0}}(q, \cdot)\right] & =0 & & \text { on } \partial \Omega \\
\int_{\partial \mathcal{S}_{0}} p_{\varepsilon}^{\partial \mathcal{S}_{0}}(q, \cdot) \mathrm{d} s & =-1 . & &
\end{aligned}
$$

Thanks to a change of variable, the identity $G(\varepsilon(x-y))=G(\varepsilon)+G(x-y)$, 8.34, 8.35 and 8.38, 8.41 can be recast as

$$
\begin{array}{cl}
S L\left[p_{\varepsilon}^{\partial \mathcal{S}_{\varepsilon}(q)}(q, \cdot)\right]+S L\left[p_{\varepsilon}^{\partial \Omega}(q, \cdot)\right]=C_{\varepsilon}(q) & \text { on } \partial \mathcal{S}_{\varepsilon}(q), \\
S L\left[p_{\varepsilon}^{\partial \mathcal{S}_{\varepsilon}(q)}(q, \cdot)\right]+S L\left[p_{\varepsilon}^{\partial \Omega}(q, \cdot)\right]=0 & \text { on } \partial \Omega, \\
\int_{\partial \mathcal{S}_{\varepsilon}(q)} p_{\varepsilon}^{\partial \mathcal{S}_{\varepsilon}(q)}(q, \cdot) \mathrm{d} s=-1 . &
\end{array}
$$

In particular we infer from 8.42a and 8.42b that, when $\psi_{\varepsilon}(q, \cdot)$ is given by 8.39 with $\mathfrak{p}_{\varepsilon}(q, \cdot)=\left(p_{\varepsilon}^{\partial \mathcal{S}_{0}}(q, \cdot), p_{\varepsilon}^{\partial \Omega}(q, \cdot), C_{\varepsilon}(q)\right)$ solution to 8.37), the boundary conditions 8.14b and 8.14c are satisfied. Moreover, by uniqueness of the solutions to the Poisson problem:

$$
\Delta \Psi=0 \text { in } \mathcal{S}_{\varepsilon}(q), \quad \Psi=C_{\varepsilon}(q) \text { on } \partial \mathcal{S}_{\varepsilon}(q),
$$

the right hand side of 8.39 is equal to $C_{\varepsilon}(q)$ in $\mathcal{S}_{\varepsilon}(q)$.

The single-layer potential $S L\left[p_{\varepsilon}^{\partial \Omega}(q, \cdot)\right]$ is smooth in a neighborhood of $\partial \mathcal{S}_{\varepsilon}(q)$. Hence, according to 8.2 , when $\psi^{\varepsilon}(q, \cdot)$ is given by 8.39 , the density $p_{\varepsilon}^{\partial \mathcal{S}_{\varepsilon}(q)}(q, \cdot)$ is equal to the jump across $\partial \mathcal{S}_{\varepsilon}(q)$ of the normal derivatives of the function equal to $\psi_{\varepsilon}(q, \cdot)$ in $\mathcal{F}_{\varepsilon}(q)$ and to $C_{\varepsilon}(q)$ in $\mathcal{S}_{\varepsilon}(q)$, that is

$$
p_{\varepsilon}^{\partial \mathcal{S}_{\varepsilon}(q)}(q, \cdot)=\frac{\partial \psi_{\varepsilon}}{\partial n}(q, \cdot) \text { on } \partial \mathcal{S}_{\varepsilon}(q) .
$$

Hence we obtain 8.40 by using 8.38 and the condition $8.14 \mathrm{~d}$ by using $8.42 \mathrm{c}$. This concludes the proof of Lemma 8.6 . 


\subsubsection{Second Step. Construction of an approximate solution}

In this step we describe an approximation $\mathfrak{p}_{\text {app }}$ up to order $O\left(\varepsilon^{3}\right)$ of the solution $\mathfrak{p}_{\varepsilon}$ of (8.37) and reformulate the equation 8.37) in terms of the rest $\mathfrak{p}_{\varepsilon}-\mathfrak{p}_{\text {app }}$. We first introduce the various terms involved in the approximation.

Densities on $\partial \mathcal{S}_{0}$. Recall that the functions $\psi_{-1}^{\mathcal{S}_{0}}, \psi_{0}^{\mathcal{S}_{0}}(q, \cdot)$ and $\psi_{1}^{\mathcal{S}_{0}}(q, \cdot)$, defined respectively in (4.5), 8.17) and (8.26), are harmonic in $\mathbb{R}^{2} \backslash \mathcal{S}_{0}$.

Let $\mathrm{p}_{-1}^{\mathcal{S}_{0}}, \mathrm{p}_{0}^{\mathcal{S}_{0}}(q, \cdot)$ and $\mathrm{p}_{1}^{\mathcal{S}_{0}}(q, \cdot)$ be the densities on $\partial \mathcal{S}_{0}$ associated respectively with $\psi_{-1}^{\mathcal{S}_{0}}, \psi_{0}^{\mathcal{S}_{0}}(q, \cdot)$ and $\psi_{1}^{\mathcal{S}_{0}}(q, \cdot)$ as explained in Propositions 8.1 and 8.2. Thus, in $\mathbb{R}^{2} \backslash \mathcal{S}_{0}$ :

$$
\psi_{-1}^{\mathcal{S}_{0}}=S L\left[\mathrm{p}_{-1}^{\mathcal{S}_{0}}\right] \quad \text { and } \quad \psi_{j}^{\mathcal{S}_{0}}(q, \cdot)=S L\left[\mathrm{p}_{j}^{\mathcal{S}_{0}}(q, \cdot)\right] \quad(j=0,1) .
$$

The single layer potentials, as being supported by $\partial \mathcal{S}_{0}$, are actually defined in $\mathbb{R}^{2}$ and harmonic in $\mathbb{R}^{2} \backslash \partial \mathcal{S}_{0}$. The identities above can therefore be used to extend the functions $\psi_{-1}^{\mathcal{S}_{0}}, \psi_{0}^{\mathcal{S}_{0}}(q, \cdot)$ and $\psi_{1}^{\mathcal{S}_{0}}(q, \cdot)$ in $\mathcal{S}_{0}$. More explicitly $\psi_{-1}^{\mathcal{S}_{0}}$ is extended by $C^{\mathcal{S}_{0}}$ in $\mathcal{S}_{0}, \psi_{0}^{\mathcal{S}_{0}}(q, \cdot)$ is extended by $P_{0}(q, \cdot)$ in $\mathcal{S}_{0}$ and $\psi_{1}^{\mathcal{S}_{0}}(q, \cdot)$ is extended by $P_{1}(q, \cdot)$ in $\mathcal{S}_{0}$, see the definitions (8.15) and 8.22 of the harmonic polynomials $P_{0}$ and $P_{1}$. Moreover using 8.2

$$
\mathbf{p}_{0}^{\mathcal{S}_{0}}=\frac{\partial \psi_{0}^{\mathcal{S}_{0}}}{\partial n}-\frac{\partial P_{0}}{\partial n} \text { and } \mathbf{p}_{1}^{\mathcal{S}_{0}}=\frac{\partial \psi_{1}^{\mathcal{S}_{0}}}{\partial n}-\frac{\partial P_{1}}{\partial n}
$$

Densities on $\partial \Omega$. We follow the same ideas to extend in $\mathbb{R}^{2}$ the functions $\psi_{0}^{\Omega}(h, \cdot), \psi_{1}^{\Omega}(q, \cdot)$ and $\psi_{2}^{\Omega}(q, \cdot)$, so far defined and harmonic in $\Omega$ (see 2.41), (8.18) and (8.48)). We consider their densities $\mathrm{p}_{0}^{\Omega}(q, \cdot), \mathrm{p}_{1}^{\Omega}(q, \cdot)$ and $\mathrm{p}_{2}^{\Omega_{2}}(q, \cdot)$, supported in $\partial \Omega$ and such that, in $\Omega$ :

$$
\psi_{0}^{\Omega}(h, \cdot)=S L\left[\mathbf{p}_{0}^{\Omega}(h, \cdot)\right], \quad \psi_{j}^{\Omega}(q, \cdot)=S L\left[\mathbf{p}_{j}^{\Omega}(q, \cdot)\right] \quad(j=1,2) .
$$

The single layer potentials being defined in $\mathbb{R}^{2}$ and harmonic $\mathbb{R}^{2} \backslash \partial \Omega$, these identities are used to extend the functions $\psi_{0}^{\Omega}(h, \cdot), \psi_{1}^{\Omega}(q, \cdot)$ and $\psi_{2}^{\Omega}(q, \cdot)$ in $\mathbb{R}^{2}$. More explicitly $\psi_{0}^{\Omega}(h, \cdot)$ is extended by $G(\cdot-h)$ in $\mathbb{R}^{2} \backslash \Omega, \psi_{1}^{\Omega}(q, \cdot)$ is extended by $-(\nabla G)(\cdot-h) \cdot \zeta_{\vartheta}$ in $\mathbb{R}^{2} \backslash \Omega$, and $\psi_{2}^{\Omega}(q, \cdot)$ is extended by $Q_{2}(q, \cdot)$, defined in $(8.46)$, in $\mathbb{R}^{2} \backslash \Omega$.

Definition of $\psi_{2}^{\Omega}(q, \cdot)$ and of $Q_{2}(q, \cdot)$. To define $\psi_{2}^{\Omega}(q, \cdot)$, we introduce the harmonic function in $\mathbb{R}^{2} \backslash \Omega$ :

$$
\begin{aligned}
Q_{2}(q, x):=\frac{1}{2}\left\langle R(\vartheta)^{t} D^{2} G(x-h)\right. & \left.R(\vartheta), T^{2}\left(\mathrm{p}_{-1}^{\mathcal{S}_{0}}\right)\right\rangle_{\mathbb{R}^{2 \times 2}} \\
& +R(\vartheta)^{t} \nabla G(x-h) \cdot T^{1}\left(\mathrm{p}_{O}^{\mathcal{S}_{0}}(q, \cdot)\right),
\end{aligned}
$$

where

$$
T^{1}\left(\mathrm{p}_{O}^{\mathcal{S}_{0}}(q, \cdot)\right):=\int_{\partial \mathcal{S}_{0}} X \mathrm{p}_{O}^{\mathcal{S}_{0}}(q, X) \mathrm{d} s(X)
$$


Then we consider $\psi_{2}^{\Omega}(q, \cdot)$ as the solution to

$$
\begin{aligned}
-\Delta \psi_{2}^{\Omega}(q, \cdot) & =0 & & \text { in } \Omega, \\
\psi_{2}^{\Omega}(q, \cdot) & =Q_{2}(q, \cdot) & & \text { on } \partial \Omega,
\end{aligned}
$$

extended by $Q_{2}(q, x)$ for $x$ in $\mathbb{R}^{2} \backslash \Omega$. These functions $\psi_{2}^{\Omega}(q, \cdot)$ and $Q_{2}(q, \cdot)$ do not appear in the claim of Proposition 8.4 and of Lemma 6.4 but will be useful later. With the choices above we aim at constructing a solution to (8.37) with $p_{\varepsilon}^{\partial \mathcal{S}_{0}}(\varepsilon, q, \cdot)$ and $p_{\varepsilon}^{\partial \Omega}(\varepsilon, q, \cdot)$ close respectively to

$$
\begin{aligned}
& \mathrm{p}_{\text {app }}^{\partial \mathcal{S}_{0}}(\varepsilon, q, \cdot):=\mathrm{p}_{-1}^{\mathcal{S}_{0}}+\varepsilon \mathrm{p}_{0}^{\mathcal{S}_{0}}(q, \cdot)+\varepsilon^{2} \mathrm{p}_{1}^{\mathcal{S}_{0}}(q, \cdot), \\
& \mathrm{p}_{\text {app }}^{\partial \Omega}(\varepsilon, q, \cdot):=\mathrm{p}_{0}^{\Omega}(h, \cdot)+\varepsilon \mathrm{p}_{1}^{\Omega}(q, \cdot)+\varepsilon^{2} \mathrm{p}_{2}^{\Omega}(q, \cdot) .
\end{aligned}
$$

The corresponding approximation $C_{\text {app }}(\varepsilon, q)$ of $C_{\varepsilon}(q)$ is chosen as:

$$
C_{\text {app }}(\varepsilon, q):=-G(\varepsilon)+\mathrm{C}_{0}(h)+\varepsilon \mathrm{C}_{1}(q)+\varepsilon^{2} \mathrm{C}_{2}(q),
$$

with

$$
\begin{aligned}
\mathrm{C}_{0}(h):= & C^{\mathcal{S}_{0}}+\psi_{0}^{\Omega}(h, h), \\
\mathrm{C}_{1}(q):= & 2 D_{x} \psi_{0}^{\Omega}(h, h) \cdot \zeta_{\vartheta}, \\
\mathrm{C}_{2}(q):= & \psi_{2}^{\Omega}(q, h)+D_{x} \psi_{1}^{\Omega}(q, h) \cdot \zeta_{\vartheta} \\
& +\frac{1}{2}\left\langle R(\vartheta)^{t} D_{x}^{2} \psi_{0}^{\Omega}(h, h) R(\vartheta), T^{2}\left(\mathrm{p}_{-1}^{\mathcal{S}_{0}}\right)\right\rangle_{\mathbb{R}^{2 \times 2}} .
\end{aligned}
$$

Using that $\psi_{0}^{\Omega}$ is symmetric with respect to its two arguments (see 8.21)), and using 2.43, we see that the first terms of the expansion above are the same as those claimed in Lemma 6.4 that is

$$
\mathrm{C}_{0}(h):=C^{\mathcal{S}_{0}}+2 \psi^{\Omega}(h) \text { and } \mathrm{C}_{1}(q):=2 \psi_{c}(q) .
$$

We finally define

$$
\mathfrak{p}_{\mathrm{app}}(\varepsilon, q, \cdot):=\left(\mathrm{p}_{\mathrm{app}}^{\partial \mathcal{S}_{0}}(\varepsilon, q, \cdot), \mathrm{p}_{\mathrm{app}}^{\partial \Omega}(\varepsilon, q, \cdot), C_{\mathrm{app}}(\varepsilon, q)\right) .
$$

Now the equation 8.37) translates as follows. Let us introduce $g^{\partial \mathcal{S}_{0}}(\varepsilon, q, \cdot)$ and $g^{\partial \Omega}(\varepsilon, q, \cdot)$ two functions respectively defined on $\partial \mathcal{S}_{0}$ and $\partial \Omega$, for $q=$ $(\vartheta, h)$, by

$$
\begin{aligned}
-g^{\partial \mathcal{S}_{0}}(\varepsilon, q, \cdot):= & \sum_{j=0}^{2} \int_{\partial \Omega} \mathrm{p}_{j}^{\Omega}(q, y) \eta_{3-j}(\varepsilon, q, \cdot, y) \mathrm{d} s(y), \\
-g^{\partial \Omega}(\varepsilon, q, x):= & \int_{\partial \mathcal{S}_{0}} \mathrm{p}_{-1}^{\mathcal{S}_{0}}(y) \eta_{3}(\varepsilon,(\vartheta, x),-y, h) \mathrm{d} s(y) \\
& +\sum_{j=0}^{1} \int_{\partial \mathcal{S}_{0}} \mathrm{p}_{j}^{\mathcal{S}_{0}}(q, y) \eta_{2-j}(\varepsilon,(\vartheta, x),-y, h) \mathrm{d} s(y),
\end{aligned}
$$


where, for $N \geqslant 1$,

$$
\eta_{N}(\varepsilon, q, \cdot, y):=\int_{0}^{1} \frac{(1-\sigma)^{N-1}}{(N-1) !} D^{N} G(\sigma \varepsilon R(\vartheta) \cdot+h-y) \cdot(R(\vartheta) \cdot)^{\otimes N} \mathrm{~d} \sigma .
$$

Let

$$
\mathfrak{g}(\varepsilon, q, \cdot):=\left(g^{\partial \mathcal{S}_{0}}(\varepsilon, q, \cdot), g^{\partial \Omega}(\varepsilon, q, \cdot), 0\right) .
$$

We can deduce from the definitions of the densities $\mathbf{p}_{j}^{\Omega}$ for $j=0,1,2, \mathrm{p}_{-1}^{\mathcal{S}_{0}}$ and $\mathrm{p}_{j}^{\mathcal{S}_{0}}$ for $j=0$ and 1 , and from Lemma 8.3 . (iii) that $g^{\partial \mathcal{S}_{0}}(\varepsilon, q, \cdot)$ and $g^{\partial \Omega}(\varepsilon, q, \cdot)$ belong respectively to $H^{1}\left(\partial \mathcal{S}_{0}\right)$ and to $H^{1}(\partial \Omega)$. Actually we even have

$$
\mathfrak{g} \in L^{\infty}\left(\mathfrak{Q}^{\delta} ; F_{1}\right) .
$$

We can now state the result of this second step.

Lemma 8.7. For any $(\varepsilon, q)$ in $\mathfrak{Q}$, let $\mathfrak{p}_{r}(\varepsilon, q, \cdot)$ in $F_{0}$ satisfy:

$$
\mathfrak{A}(\varepsilon, q)\left[\mathfrak{p}_{r}(\varepsilon, q, \cdot)\right]=\mathfrak{g}(\varepsilon, q, \cdot) .
$$

Then

$$
\mathfrak{p}_{\varepsilon}(q, \cdot):=\mathfrak{p}_{\text {app }}(\varepsilon, q, \cdot)+\varepsilon^{3} \mathfrak{p}_{r}(\varepsilon, q, \cdot),
$$

is solution to 8.37 .

Let us stress in particular that the third coordinate of the left hand side of 8.58 does not contain the singular term $G(\varepsilon)$ anymore (compare with 8.37) and that the third coordinate of the right hand side of 8.58 is now 0 .

Proof Let $(\varepsilon, q)$ in $\mathfrak{Q}$ and $\mathfrak{p}_{r}(\varepsilon, q, \cdot):=\left(\mathrm{p}_{r}^{\partial \mathcal{S}_{0}}(\varepsilon, q, \cdot), p_{r}^{\partial \Omega}(\varepsilon, q, \cdot), C_{r}(\varepsilon, q)\right)$ in $F_{0}$ satisfying (8.58) (where $\mathfrak{A}$ is defined in (8.36)), that is

$$
\begin{gathered}
S L\left[\mathrm{p}_{r}^{\partial \mathcal{S}_{0}}(\varepsilon, q, \cdot)\right]+K^{\partial \mathcal{S}_{0}}(\varepsilon, q)\left[\mathrm{p}_{r}^{\partial \Omega}(\varepsilon, q, \cdot)\right]-C_{r}(\varepsilon, q)=g^{\partial \mathcal{S}_{0}}(\varepsilon, q, \cdot) \text { on } \partial \mathcal{S}_{0}, \\
S L\left[\mathrm{p}_{r}^{\partial \Omega}(\varepsilon, q, \cdot)\right]+K^{\partial \Omega}(\varepsilon, q)\left[\mathrm{p}_{r}^{\partial \mathcal{S}_{0}}(\varepsilon, q, \cdot)\right]=g^{\partial \Omega}(\varepsilon, q, \cdot) \text { on } \partial \Omega \\
\int_{\partial \mathcal{S}_{0}} \mathrm{p}_{r}^{\partial \mathcal{S}_{0}}(\varepsilon, q, \cdot) \mathrm{d} s=0 .
\end{gathered}
$$

Let $\mathfrak{p}_{\varepsilon}(q, \cdot)=\left(p_{\varepsilon}^{\partial \mathcal{S}_{0}}(q, \cdot), p_{\varepsilon}^{\partial \Omega}(q, \cdot), C_{\varepsilon}(q)\right)$ in $F_{0}$ given by 8.59). To prove 8.37 we now verify the three parts of 8.41).

Let us start with (8.41c). Using the second equality in (8.6) and (8.44), the fact that $P_{0}$ and $P_{1}$ are harmonic and the conditions $\left.4.5 \mathrm{~d}, 8.17 \mathrm{c}\right)$ and 8.26c), we arrive at

$$
\int_{\partial \mathcal{S}_{0}} \mathrm{p}_{-1}^{\mathcal{S}_{0}} \mathrm{~d} s=-1 \text { and } \int_{\partial \mathcal{S}_{0}} \mathrm{p}_{0}^{\mathcal{S}_{0}}(q, \cdot) \mathrm{d} s=\int_{\partial \mathcal{S}_{0}} \mathbf{p}_{1}^{\mathcal{S}_{0}}(q, \cdot) \mathrm{d} s=0
$$

We deduce from $(8.62)$ and $(8.63)$ that the condition $8.41 \mathrm{c})$ is fulfilled. 
Let us now verify 8.41a). Using 8.53, 8.59, 8.6, 4.5b, 8.43, we obtain, on $\partial \mathcal{S}_{0}$,

$$
S L\left[p_{\varepsilon}^{\partial \mathcal{S}_{0}}(q, \cdot)\right]=C^{\mathcal{S}_{0}}+\varepsilon \psi_{0}^{\mathcal{S}_{0}}(q, \cdot)+\varepsilon^{2} \psi_{1}^{\mathcal{S}_{0}}(q, \cdot)+\varepsilon^{3} S L\left[p_{\varepsilon, r}^{\partial \mathcal{S}_{0}}(\varepsilon, q, \cdot)\right] .
$$

It follows from 8.34, 8.53, 8.59, 8.45, Taylor's formula and 8.19 that, on $\partial \mathcal{S}_{0}$,

$$
\begin{array}{r}
K^{\partial \mathcal{S}_{0}}(\varepsilon, q)\left[p_{\varepsilon}^{\partial \Omega}(q, \cdot)\right](X)=\psi_{0}^{\Omega}(h, h)+\varepsilon D_{x} \psi_{0}^{\Omega}(h, h) \cdot\left(R(\vartheta) X+\zeta_{\vartheta}\right) \\
+\varepsilon^{2}\left(\psi_{2}^{\Omega}(q, h)+D_{x} \psi_{1}^{\Omega}(q, h) \cdot R(\vartheta) X+\frac{1}{2} D_{x}^{2} \psi_{0}^{\Omega}(h, h) \cdot(R(\vartheta) X, R(\vartheta) X)\right) \\
+\varepsilon^{3}\left(K^{\partial \mathcal{S}_{0}}(\varepsilon, q)\left[\mathrm{p}_{r}^{\partial \Omega}(\varepsilon, q, \cdot)\right](X)-g^{\partial \mathcal{S}_{0}}(\varepsilon, q, X)\right) \cdot
\end{array}
$$

We recall that the function $g^{\partial \mathcal{S}_{0}}(\varepsilon, q, \cdot)$ is defined in 8.54a).

Gathering 8.50, 8.59, 8.64 and 8.65 we obtain, on $\partial \mathcal{S}_{0}$,

$$
\begin{gathered}
-G(\varepsilon)+S L\left[p_{\varepsilon}^{\partial \mathcal{S}_{0}}(q, \cdot)\right]+K^{\partial \mathcal{S}_{0}}(\varepsilon, q)\left[p_{\varepsilon}^{\partial \Omega}(q, \cdot)\right]-C_{\varepsilon}(q) \\
=\varepsilon\left(\psi_{0}^{\mathcal{S}_{0}}(q, \cdot)-P_{0}(q, \cdot)\right)+\varepsilon^{2}\left(\psi_{1}^{\mathcal{S}_{0}}(q, \cdot)-P_{1}(q, \cdot)\right) \\
+\varepsilon^{3}\left(S L\left[p_{\varepsilon, r}^{\partial \mathcal{S}_{0}}(\varepsilon, q, \cdot)\right]+K^{\partial \mathcal{S}_{0}}(\varepsilon, q)\left[\mathrm{p}_{r}^{\partial \Omega}(\varepsilon, q, \cdot)\right]-C_{r}(\varepsilon, q)-g^{\partial \mathcal{S}_{0}}(\varepsilon, q, \cdot)\right),
\end{gathered}
$$

where $P_{0}(q, \cdot)$ and $P_{1}(q, \cdot)$ are the harmonic polynomials defined respectively in 8.15 and in 8.22. Now taking into account the boundary conditions $8.17 \mathrm{~b}$ and $8.48 \mathrm{~b})$, and 8.60 we deduce that $8.41 \mathrm{a}$ holds true.

Finally we move to the verification of 8.41b. First, using 8.53), 8.59 and 8.45 , we obtain, on $\partial \Omega$,

$$
S L\left[p_{\varepsilon}^{\partial \Omega}(q, \cdot)\right]=\psi_{O}^{\Omega}(h, \cdot)+\varepsilon \psi_{1}^{\Omega}(q, \cdot)+\varepsilon^{2} \psi_{2}^{\Omega}(q, \cdot)+\varepsilon^{3} S L\left[\mathrm{p}_{r}^{\partial \Omega}(\varepsilon, q, \cdot)\right] .
$$

By 4.15, 4.13), the second equality in 8.6 and 8.23),

$$
\begin{gathered}
\int_{\partial \mathcal{S}_{0}} \mathrm{p}_{-1}^{\mathcal{S}_{0}}(X) R(\vartheta) X \mathrm{~d} s(X)=-\zeta_{\vartheta}, \\
\int_{\partial \mathcal{S}_{0}} \mathrm{p}_{-1}^{\mathcal{S}_{0}}(X) X^{\otimes 2} \mathrm{~d} s(X)=T^{2}\left(\mathrm{p}_{-1}^{\mathcal{S}_{0}}\right),
\end{gathered}
$$

On the other hand, using 8.35, 8.59, Taylor's formula, 8.54b, 8.47), 8.62, 8.63, 8.67, 8.68, we deduce, for $x$ in $\partial \Omega$,

$$
\begin{aligned}
& K^{\partial \Omega}(\varepsilon, q)\left[p_{\varepsilon}^{\partial \mathcal{S}_{0}}(q, \cdot)\right](x)=-G(x-h)+\varepsilon D G(x-h) \cdot \zeta_{\vartheta} \\
&+\varepsilon^{2}(-R(\vartheta)^{t} D G(x-h) \cdot T^{1}\left(\mathrm{p}_{0}^{\mathcal{S}_{0}}(q, \cdot)\right) \\
&\left.\quad-\frac{1}{2}\left\langle R(\vartheta)^{t} D_{x}^{2} G(x-h) R(\vartheta), T^{2}\left(\mathrm{p}_{-1}^{\mathcal{S}_{0}}\right)\right\rangle_{\mathbb{R}^{2 \times 2}}\right) \\
&+\varepsilon^{3}\left(K^{\partial \Omega}(\varepsilon, q)\left[p_{r}^{\partial \mathcal{S}_{0}}(\varepsilon, q, \cdot)\right](x)-g^{\partial \Omega}(\varepsilon, q, x)\right) .
\end{aligned}
$$


By 8.66) and (8.69), the equation 8.41b now reads, for $x$ in $\partial \Omega$,

$$
\begin{array}{r}
S L\left[p_{\varepsilon}^{\partial \Omega}(q, \cdot)\right](x)+K^{\partial \Omega}(\varepsilon, q)\left[p_{\varepsilon}^{\partial \mathcal{S}_{0}}(q, \cdot)\right](x)=\psi_{0}^{\Omega}(h, x)-G(x-h) \\
+\varepsilon\left(\psi_{1}^{\Omega}(q, x)+D G(x-h) \cdot \zeta_{\vartheta}\right)+\varepsilon^{2}\left(\psi_{2}^{\Omega}(q, x)-Q_{2}(q, x)\right) \\
+\varepsilon^{3}\left(S L\left[\mathrm{p}_{r}^{\partial \Omega}(\varepsilon, q, \cdot)\right]+K^{\partial \Omega}(\varepsilon, q)\left[p_{\varepsilon, r}^{\partial \mathcal{S}_{0}}(\varepsilon, q, \cdot)\right](x)-g^{\partial \Omega}(\varepsilon, q, x)\right),
\end{array}
$$

where $Q_{2}(q, x)$ denotes the harmonic polynomial defined in 8.46 .

Taking now the boundary conditions 2.41, $8.18 \mathrm{~b}),(8.48 \mathrm{~b}$ ) and (8.61) into account, we deduce from the equation (8.70) that (8.41b) holds true. This concludes the proof of Lemma 8.7 .

\subsubsection{Third Step. Existence and estimate of the remainders}

In this third step we prove, for $(\varepsilon, q)$ in $\mathfrak{Q}_{\delta, \varepsilon_{0}}$ with $\delta$ and $\varepsilon_{0}$ positive and small enough, the existence of $\mathfrak{p}_{r}(\varepsilon, q, \cdot)$ in $F_{0}$ satisfying (8.58) and provide an estimate in $F_{0}$, uniform over $(\varepsilon, q)$ in $\mathfrak{Q}_{\delta, \varepsilon_{0}}$.

We will make use of the fact that the the third argument of the right hand side of 8.58 vanishes. Accordingly, we define

$$
\tilde{F}_{1}:=H^{1}\left(\partial \mathcal{S}_{0}\right) \times H^{1}(\partial \Omega) \times\{0\},
$$

which is a closed subspace of $F_{1}$ and prove the following result.

Lemma 8.8. Let $\delta>0$. There exists $\varepsilon_{0}$ in $(0,1)$, such that for any $\mathfrak{g}$ in $L^{\infty}\left(\mathfrak{Q}_{\delta, \varepsilon_{0}} ; \tilde{F}_{1}\right)$, there exists $\mathfrak{p}_{r}$ in $L^{\infty}\left(\mathfrak{Q}_{\delta, \varepsilon_{0}} ; F_{0}\right)$ such that $\mathfrak{p}_{r}(\varepsilon, q, \cdot)$ solves 8.58 for any $(\varepsilon, q)$ in $\mathfrak{Q}_{\delta, \varepsilon_{0}}$.

Proof of Lemma 8.8. To prove Lemma 8.8 let us start with stating a perturbative result. We will use the notation that given $X$ and $Y$ two Banach spaces, $\mathcal{L}(X ; Y)$ is the space of bounded linear operators from $X$ to $Y$. Now the framework is as follows. Let $\delta>0$. Recall that $\mathfrak{Q}^{\delta}$ and $\Omega_{\delta}$ were defined in (6.3) and 6.5). We introduce the following families of operators.

- First we consider a family of operators in $\mathcal{L}\left(L^{2}(\partial \Omega) ; H^{1}\left(\partial \mathcal{S}_{0}\right)\right)$ :

$$
\begin{array}{r}
\tilde{K}^{\partial \mathcal{S}_{0}} \in \operatorname{Lip}\left(\overline{\Omega_{\delta}} ; \mathcal{L}\left(L^{2}(\partial \Omega) ; H^{1}\left(\partial \mathcal{S}_{0}\right)\right)\right) \text { such that for all } h \text { in } \overline{\Omega_{\delta}} \\
\tilde{K}^{\partial \mathcal{S}_{0}}(h) \text { is compact from } L^{2}(\partial \Omega) \text { to } H^{1}\left(\partial \mathcal{S}_{0}\right)
\end{array}
$$

- Next we consider two families of operators: one in $\mathcal{L}\left(L^{2}(\partial \Omega) ; H^{1}\left(\partial \mathcal{S}_{0}\right)\right)$ and the other one in $\mathcal{L}\left(L^{2}\left(\partial \mathcal{S}_{0}\right) ; H^{1}(\partial \Omega)\right)$ :

$$
\begin{aligned}
& \left(T^{\partial \mathcal{S}_{0}}(\varepsilon, q)\right)_{(\varepsilon, q) \in \mathfrak{Q}^{\delta}} \text { bounded in } \mathcal{L}\left(L^{2}(\partial \Omega) ; H^{1}\left(\partial \mathcal{S}_{0}\right)\right), \\
& \left(T^{\partial \Omega}(\varepsilon, q)\right)_{(\varepsilon, q) \in \mathfrak{Q}^{\delta}} \text { bounded in } \mathcal{L}\left(L^{2}\left(\partial \mathcal{S}_{0}\right) ; H^{1}(\partial \Omega)\right) .
\end{aligned}
$$


Given these operators we can construct the following one. For $(\varepsilon, q)$ in $\mathfrak{Q}^{\delta}$, let $A(\varepsilon, q): F_{0} \rightarrow F_{1}$ given by the following formula: for any $\mathfrak{p}:=\left(\mathrm{p}^{\partial \mathcal{S}_{0}}, \mathrm{p}^{\partial \Omega}, C\right)$ in $F_{0}$,

$$
A(\varepsilon, q)[\mathfrak{p}]:=\left(A(\varepsilon, q)[\mathfrak{p}]_{i}\right)_{1 \leqslant i \leqslant 3} \in F_{1}
$$

with

$$
\begin{aligned}
& A(\varepsilon, q)[\mathfrak{p}]_{1}:=S L\left[\mathrm{p}^{\partial \mathcal{S}_{0}}\right]+\tilde{K}^{\partial \mathcal{S}_{0}}(h)\left[\mathrm{p}^{\partial \Omega}\right]+\varepsilon T^{\partial \mathcal{S}_{0}}(\varepsilon, q)\left[\mathrm{p}^{\partial \Omega}\right]-C, \\
& A(\varepsilon, q)[\mathfrak{p}]_{2}:=S L\left[\mathrm{p}^{\partial \Omega}\right]+\varepsilon T^{\partial \Omega}(\varepsilon, q)\left[\mathrm{p}^{\partial \mathcal{S}_{0}}\right], \\
& A(\varepsilon, q)[\mathfrak{p}]_{3}:=\int_{\partial \mathcal{S}_{0}} \mathrm{p}^{\partial \mathcal{S}_{0}} \mathrm{~d} s .
\end{aligned}
$$

We will use the following perturbative result.

Lemma 8.9. Let $\delta>0$ and for $(\varepsilon, q)$ in $\mathfrak{Q}^{\delta}, A(\varepsilon, q)$ given as above, with assumptions 8.72 and 8.73 . Then there exists $\varepsilon_{0}$ in $(0,1)$ such that for any $(\varepsilon, q)$ in $\mathfrak{Q}_{\delta, \varepsilon_{0}}, A(\varepsilon, q)$ is an isomorphism from $F_{0}$ to $F_{1}$ and

$$
\sup _{(\varepsilon, q) \in \mathfrak{Q}_{\delta, \varepsilon_{0}}}\left\|A(\varepsilon, q)^{-1}\right\|_{\mathcal{L}\left(F_{1} ; F_{0}\right)}<\infty .
$$

Proof It is straightforward to see that for any $(\varepsilon, q)$ in $\mathfrak{Q}, A(\varepsilon, q)$ is linear continuous. Let $(\varepsilon, q)$ in $\mathcal{Q}$, with $q=(\vartheta, h)$ in $\mathbb{R} \times \Omega$. Let us introduce, for any $\mathfrak{p}:=\left(\mathrm{p}^{\partial \mathcal{S}_{0}}, \mathrm{p}^{\partial \Omega}, C\right)$ in $F_{-\frac{1}{2}}$,

$$
\begin{aligned}
L[\mathfrak{p}] & :=\left(S L\left[\mathrm{p}^{\partial \mathcal{S}_{0}}\right], S L\left[\mathrm{p}^{\partial \Omega}\right], C\right), \\
K(h)[\mathfrak{p}] & :=\left(\tilde{K}^{\partial \mathcal{S}_{0}}(h)\left[\mathrm{p}^{\partial \Omega}\right]-C, 0, \int_{\partial \mathcal{S}_{0}} \mathrm{p}^{\partial \mathcal{S}_{0}} \mathrm{~d} s-C\right), \\
T(\varepsilon, q)[\mathfrak{p}] & :=\left(T^{\partial \mathcal{S}}(\varepsilon, q)\left[\mathrm{p}^{\partial \Omega}\right], T^{\partial \Omega}(\varepsilon, q)\left[\mathrm{p}^{\partial \mathcal{S}_{0}}\right], 0\right),
\end{aligned}
$$

so that we can write $A$ in the following form: on $F_{0}$,

$$
A(\varepsilon, q)=L+K(h)+\varepsilon T(\varepsilon, q) .
$$

We first consider the operator $L+K(h)$. According to 8.3 , the operator $L$ is Fredholm with index zero and since for each $h$ in $\overline{\Omega_{\delta}}, K(h)$ is compact, we deduce that $L+K(h)$ is Fredholm with index zero. It follows that to prove that $L+K(h)$ is an isomorphism, it is sufficient to prove that its kernel is trivial.

Consider $\mathfrak{p}:=\left(\mathrm{p}^{\partial \mathcal{S}_{0}}, \mathrm{p}^{\partial \Omega}, C\right)$ in $F_{-\frac{1}{2}}$ such that $(L+K(h))[\mathfrak{p}]=0$. Since the logarithmic capacity $\operatorname{Cap}(\partial \Omega)$ of $\partial \Omega$ satisfies $\operatorname{Cap}(\partial \Omega) \neq 1$, according to (8.5), the second equation $S L\left[\mathrm{p}^{\partial \Omega}\right]=0$ implies $\mathrm{p}^{\partial \Omega}=0$. Then, substituting into the first equation, $S L\left[\mathrm{p}^{\mathcal{S}_{0}}\right]=C$, whereas the third equation reads $\int_{\partial \mathcal{S}_{0}} \mathrm{p}^{\partial \mathcal{S}_{0}} \mathrm{~d} s=0$. Thus according to 8.4 , we obtain $\mathrm{p}^{\partial \mathcal{S}_{0}}=0$ and thus $C=0$. This proves that the kernel of $L+\bar{K}(h)$ is trivial, and consequently that for any $h$ in $\Omega_{\delta}$, $L+K(h)$ is an isomorphism. 
Now using that the dependence of $K$ on $h$ is Lipschitz, we deduce that $L+K(h)$ has locally a bounded inverse. By compactness of $\overline{\Omega_{\delta}}$, it follows that $L+K(h)$ has a bounded inverse for $h$ running over $\overline{\Omega_{\delta}}$.

Since the operators $\left(T_{\varepsilon}\right)_{\varepsilon \in(0,1)}$ are bounded in the space of bounded operators from $F_{0}$ to $F_{1}$ we can then easily deduce the result from (8.77). This concludes the proof of Lemma 8.9 .

In our case, Lemma 8.9 is applied as follows. Recalling 8.34-8.35 we define, for any $(\varepsilon, q)$ in $\mathfrak{Q}$, with $q=(\vartheta, h)$,

- for any density $\mathrm{p}^{\partial \Omega}$ in $L^{2}(\partial \Omega)$,

$$
\tilde{K}^{\partial \mathcal{S}_{0}}(h)\left[\mathrm{p}^{\partial \Omega}\right]=K^{\partial \mathcal{S}_{0}}(0,0, h)\left[\mathrm{p}^{\partial \Omega}\right]=S L\left[\mathrm{p}^{\partial \Omega}\right](h)
$$

as a constant function on $\partial \mathcal{S}_{0}$, and

$$
T^{\partial \mathcal{S}_{0}}(\varepsilon, q)\left[\mathrm{p}^{\partial \Omega}\right]:=\int_{\partial \Omega} \mathrm{p}^{\partial \Omega}(y) \eta_{1}(\varepsilon, q, \cdot, y) \mathrm{d} s(y) \text { on } \partial \mathcal{S}_{0},
$$

- for any density $\mathrm{p}^{\partial \mathcal{S}_{0}}$ in $L^{2}\left(\partial \mathcal{S}_{0}\right)$,

$$
T^{\partial \Omega}(\varepsilon, q)\left[\mathrm{p}^{\partial \mathcal{S}_{0}}\right]:=\int_{\partial \mathcal{S}_{0}} \mathrm{p}^{\partial \mathcal{S}_{0}}(Y) \eta_{1}(\varepsilon, \vartheta, \cdot,-Y, h) \mathrm{d} s(y) \text { on } \partial \Omega .
$$

The following lemma entails that the hypotheses of Lemma 8.9 are satisfied.

Lemma 8.10. Let $\delta>0$. With the definitions above, 8.72 and 8.73 hold true.

Proof We use Lemma 8.3 with $\mathcal{C}=\partial \Omega, b=G$ and $\mathrm{p}^{\mathcal{C}}=\mathrm{p}^{\partial \Omega}$ to obtain that $\tilde{K}^{\partial \mathcal{S}_{0}}$ satisfies 8.72 . Next we apply Lemma 8.3 (ii) for any $(\varepsilon, q)$ in $\mathfrak{Q}_{\delta, \varepsilon_{0}}$, with $\mathcal{C}=\partial \Omega, \bar{b}(x, y)=\eta_{1}(\varepsilon, q, x, y)$ and $\mathrm{p}^{\mathcal{C}}=\mathrm{p}^{\partial} \Omega$ and with $\mathcal{C}=\partial \mathcal{S}_{0}$, $b(x, y)=\eta_{1}(\varepsilon, \vartheta, x,-y, h)$ and $\mathrm{p}^{\mathcal{C}}=\mathrm{p}_{\mathcal{S}_{0}}$ to get that $T^{\partial \mathcal{S}_{0}}(\varepsilon, q)$ and $T^{\partial \Omega}(\varepsilon, q)$ satisfy 8.73 . This ends the proof of Lemma 8.10

Then we consider the operator $A(\varepsilon, q)$ associated with these operators $\tilde{K}^{\partial \mathcal{S}_{0}}(h), T^{\partial \mathcal{S}_{0}}(\varepsilon, q)$ and $T^{\partial \Omega}(\varepsilon, q)$ as given by 8.74 - 8.75). The next lemma shows that this operator $A(\varepsilon, q)$ provides the existence of a solution to 8.58 with uniform estimates.

Lemma 8.11. Let $\delta>0$. There exists $\varepsilon_{0}$ in $(0,1)$ such that for any $(\varepsilon, q)$ in $\mathfrak{Q}_{\delta, \varepsilon_{0}}$,

$$
\mathfrak{p}_{r}(\varepsilon, q, \cdot):=A(\varepsilon, q)^{-1} \mathfrak{g}(\varepsilon, q, \cdot)
$$

belongs to $F_{0}$ and solves 8.58). Moreover $\mathfrak{p}_{r}$ is in $L^{\infty}\left(\mathfrak{Q}_{\delta, \varepsilon_{0}} ; F_{0}\right)$.

Proof Let $\delta>0$. Let us first observe that for any $(\varepsilon, q)$ in $\mathfrak{Q}_{\delta}$, for any $\mathfrak{p}:=$ $\left(\mathrm{p}^{\partial \mathcal{S}_{0}}, \mathrm{p}^{\partial \Omega}, C\right)$ in $F_{0}$ satisfying the condition

$$
\int_{\partial \mathcal{S}_{0}} \mathrm{p}^{\partial \mathcal{S}_{0}} \mathrm{~d} s=0
$$


the following equality holds:

$$
\mathfrak{A}(\varepsilon, q)[\mathfrak{p}]=A(\varepsilon, q)[\mathfrak{p}] .
$$

Indeed 8.78 and first order Taylor expansions yield with 8.79 and 8.80 that

$$
\begin{aligned}
K^{\partial \mathcal{S}_{0}}(\varepsilon, q)\left[\mathrm{p}^{\partial \Omega}\right]-\tilde{K}^{\partial \mathcal{S}_{0}}(h)\left[\mathrm{p}^{\partial \Omega}\right] & =\varepsilon T^{\partial \mathcal{S}_{0}}(\varepsilon, q)\left[\mathrm{p}^{\partial \Omega}\right] \\
K^{\partial \Omega}(\varepsilon, q)\left[\mathrm{p}^{\partial \mathcal{S}_{0}}\right] & =\varepsilon T^{\partial \Omega}(\varepsilon, q)\left[\mathrm{p}^{\partial \mathcal{S}_{0}}\right] .
\end{aligned}
$$

We emphasize in particular that the last equality relies on the condition 8.82).

Now, consider $\varepsilon_{0}$ in $(0,1)$ obtained by applying Lemma 8.9 . For any $(\varepsilon, q)$ in $\mathfrak{Q}_{\delta, \varepsilon_{0}}$, consider $\mathfrak{p}_{r}(\varepsilon, q, \cdot)=\left(\mathrm{p}_{r}^{\partial \mathcal{S}_{0}}(\varepsilon, q, \cdot), p_{r}^{\partial \Omega}(\varepsilon, q, \cdot), C_{r}(\varepsilon, q)\right)$ given by 8.81). It belongs to $F_{0}$ and satisfies 8.62 and consequently

$$
\mathfrak{A}(\varepsilon, q)\left[p_{r}(\varepsilon, q, \cdot)\right]=A(\varepsilon, q)\left[p_{r}(\varepsilon, q, \cdot)\right]=\mathfrak{g}(\varepsilon, q, \cdot) .
$$

Moreover we have the estimate

$$
\left\|p_{r}(\varepsilon, q, \cdot)\right\|_{F_{0}} \leqslant\left\|A(\varepsilon, q)^{-1}\right\|_{\mathcal{L}\left(F_{1} ; F_{0}\right)}\|\mathfrak{g}(\varepsilon, q, \cdot)\|_{F_{1}} .
$$

The estimates 8.57) and 8.76 entail that $\mathfrak{p}_{r}$ is in $L^{\infty}\left(Q_{\delta, \varepsilon_{0}} ; F_{0}\right)$, which concludes the proof of Lemma 8.11

Lemma 8.8 follows in a straightforward manner.

\subsubsection{Fourth Step. Conclusion}

End of proof of Lemma 6.4 We apply Lemma 8.8 to 8.56). Thanks to 8.57) the assumption is satisfied. Regarding $C_{\varepsilon}(q)$ this yields an expansion actually better than the one stated in Lemma 6.4. that is, according to 8.50 and 8.52 and what precedes, there exists $C_{r}$ in $L^{\infty}\left(\mathfrak{Q}_{\delta, \varepsilon_{0}} ; \mathbb{R}\right)$ such that

$$
\begin{aligned}
C_{\varepsilon}(q)=-G(\varepsilon)+C^{\mathcal{S}_{0}}+\psi_{0}^{\Omega}(h, h)+2 \varepsilon D_{x} \psi_{0}^{\Omega}(h, h) \cdot \zeta_{\vartheta} & +\varepsilon^{2} C_{2}(q) \\
& +\varepsilon^{3} C_{r}(\varepsilon, q),
\end{aligned}
$$

where $\mathrm{C}_{2}(q)$ is given by 8.51 . To prove Lemma 6.4 it is therefore sufficient to observe that $\mathrm{C}_{2}(q)$ is bounded uniformly in $\mathbb{R}$ for $(\varepsilon, q)$ in $\mathfrak{Q}^{\delta}$ and to redefine $C_{r}(\varepsilon, q)$ such that $\varepsilon^{2} C_{r}(\varepsilon, q)$ is equal to the sum of the last two terms in 8.83 .

End of proof of Proposition 8.4 Combining 8.40, 8.49) and 8.59, we deduce that on $\partial \mathcal{S}_{0}$

$$
\frac{\partial \psi_{\varepsilon}}{\partial n}(q, \varepsilon R(\vartheta) \cdot+h)=\mathrm{p}_{-1}^{\mathcal{S}_{0}}(\cdot)+\varepsilon \mathrm{p}_{0}^{\mathcal{S}_{0}}(q, \cdot)+\varepsilon^{2} \mathrm{p}_{1}^{\mathcal{S}_{0}}(q, \cdot)+\varepsilon^{3} \mathrm{p}_{r}^{\partial \mathcal{S}_{0}}(\varepsilon, q, \cdot),
$$

with $\mathrm{p}_{r}^{\partial \mathcal{S}_{0}}$ in $L^{\infty}\left(\mathfrak{Q}_{\delta, \varepsilon_{0}} ; L^{2}\left(\partial \mathcal{S}_{0} ; \mathbb{R}\right)\right)$.

Combining this with the second equality in 8.6, 8.44 and using that $\frac{\partial P_{0}}{\partial n}(q, X)=-R(\vartheta)^{t} u^{\Omega}(h) \cdot \tau$, for $X$ on $\partial \mathcal{S}_{0}$, as a direct consequence of the definition of $P_{0}$ in 8.15 , we conclude the proof of Proposition 8.4. 
8.4 Asymptotic expansion of the potential part: Proof of Proposition 8.5

The proof of Proposition 8.5 is very close to the one of Proposition 8.4. We will only explain how to transform the (Neumann) problem defining the Kirchhoff potentials into a Dirichlet one, so that the proof of Proposition 8.5 follows from a tedious adaptation of the steps of the proof of Proposition 8.4 detailed in Subsection 8.3 .

We emphasize that the indices below correspond to coordinates in $\mathbb{R}^{3}$ and are in normal font type (while indices related to the order in an asymptotic development in powers of $\varepsilon$ are written in italic type).

We consider the functions $\bar{\varphi}_{j, \varepsilon}(q, \cdot)$, for $j=1,2,3$, as the solution to the following Dirichlet boundary value problem in $\mathcal{F}_{\varepsilon}(q)$ :

$$
\begin{aligned}
-\Delta \bar{\varphi}_{j, \varepsilon}(q, \cdot) & =0 & & \text { in } \mathcal{F}_{\varepsilon}(q), \\
\bar{\varphi}_{j, \varepsilon}(q, \cdot) & =\bar{K}_{j}(q, \cdot)+c_{j, \varepsilon}(q) & & \text { on } \partial \mathcal{S}_{\varepsilon}(q), \\
\bar{\varphi}_{j, \varepsilon}(q, \cdot) & =0 & & \text { on } \partial \Omega,
\end{aligned}
$$

where the functions $\bar{K}_{j}(q, \cdot)$ are given by

$$
\bar{K}_{j}(q, \cdot):=\left\{\begin{array}{cl}
\frac{1}{2}|x-h|^{2} & \text { if } j=1, \\
-R(\vartheta)^{t}(x-h) \cdot e_{2} & \text { if } j=2, \\
R(\vartheta)^{t}(x-h) \cdot e_{1} & \text { if } j=3,
\end{array}\right.
$$

where $e_{1}$ and $e_{2}$ are the unit vectors of the canonical basis, and the constants $c_{j, \varepsilon}(q)$ are such that:

$$
\int_{\partial \mathcal{S}_{\varepsilon}(q)} \frac{\partial \bar{\varphi}_{j, \varepsilon}}{\partial n}(q, \cdot) \mathrm{d} s=0 .
$$

Precisely, the constants $c_{j, \varepsilon}(q)$ are given by

$$
c_{j, \varepsilon}(q)=C_{\varepsilon}(q) \int_{\partial \mathcal{S}_{\varepsilon}(q)} \frac{\partial \bar{\phi}_{j, \varepsilon}}{\partial n}(q, \cdot) \mathrm{d} s,
$$

where $\bar{\phi}_{j, \varepsilon}, j=1,2,3$ are the solutions of

$$
\begin{aligned}
-\Delta \bar{\phi}_{j, \varepsilon}(q, \cdot) & =0 & & \text { in } \mathcal{F}_{\varepsilon}(q), \\
\bar{\phi}_{j, \varepsilon}(q, \cdot) & =\bar{K}_{j}(q, \cdot) & & \text { on } \partial \mathcal{S}_{\varepsilon}(q), \\
\bar{\phi}_{j, \varepsilon}(q, \cdot) & =0 & & \text { on } \partial \Omega .
\end{aligned}
$$

We will use the vector notation:

$$
\bar{\varphi}_{\varepsilon}:=\left(\bar{\varphi}_{1, \varepsilon}, \bar{\varphi}_{2, \varepsilon}, \bar{\varphi}_{3, \varepsilon}\right)^{t} .
$$

Up to a rotation, the functions $\bar{\varphi}_{j, \varepsilon}(q, \cdot)$ are harmonically conjugated to the Kirchhoff potentials $\varphi_{j, \varepsilon}(q, \cdot)$ (see 8.29$)$ ), as shown in the following result. 
Lemma 8.12. For any $(\varepsilon, q)$ in $\mathfrak{Q}$, with $q=(\vartheta, h)$, there holds in $\mathcal{F}_{\varepsilon}(q)$,

$$
\nabla \varphi_{j, \varepsilon}(q, \cdot)=\nabla^{\perp} \check{\varphi}_{j, \varepsilon}(q, \cdot) \text {, }
$$

where

$$
\left(\check{\varphi}_{1, \varepsilon}(q, \cdot), \check{\varphi}_{2, \varepsilon}(q, \cdot), \check{\varphi}_{3, \varepsilon}(q, \cdot)\right):=\mathcal{R}(\vartheta)\left(\bar{\varphi}_{1, \varepsilon}(q, \cdot), \bar{\varphi}_{2, \varepsilon}(q, \cdot), \bar{\varphi}_{3, \varepsilon}(q, \cdot)\right) .
$$

Proof We recall that for any $(\varepsilon, q)$ in $\mathfrak{Q}$, the system

$$
\begin{aligned}
\operatorname{div} u & =0 & & \text { in } \mathcal{F}_{\varepsilon}(q), \\
\operatorname{curl} u & =0 & & \text { in } \mathcal{F}_{\varepsilon}(q), \\
u \cdot n & =0 & & \text { on } \partial \Omega, \\
u \cdot n & =K_{j}(q, \cdot) & & \text { on } \partial \mathcal{S}_{\varepsilon}(q), \\
\int_{\partial \mathcal{S}_{\varepsilon}(q)} u \cdot \tau \mathrm{d} s & =0, & &
\end{aligned}
$$

has a unique solution $u$, say in $H^{1}\left(\mathcal{F}_{\varepsilon}(q)\right)$. Then one observes that both $\nabla \varphi_{j, \varepsilon}(q, \cdot)$ and $\nabla^{\perp} \check{\varphi}_{j, \varepsilon}(q, \cdot)$ solve 8.92 . In particular let us emphasize that, on $\partial \mathcal{S}_{\varepsilon}(q)$,

$$
\left(n \cdot \nabla^{\perp} \bar{\varphi}_{j, \varepsilon}(q, \cdot)\right)_{j=1,2,3}=\left(\frac{\partial \bar{K}_{j}}{\partial \tau}(q, \cdot)\right)_{j=1,2,3}=\mathcal{R}(\vartheta)^{t} \boldsymbol{K}(q, \cdot),
$$

so that, for $j=1,2,3, \nabla^{\perp} \check{\varphi}_{j, \varepsilon}(q, \cdot)$ satisfies 8.92d), and the condition 8.84d ensures that $8.92 \mathrm{e}$ is satisfied.

In the case without exterior boundary we consider in the same way $\bar{\varphi}_{j}^{\mathcal{S}_{0}}$ as the solution to

$$
\begin{aligned}
-\Delta \bar{\varphi}_{j}^{\mathcal{S}_{0}} & =0 & & \text { in } \mathbb{R}^{2} \backslash \mathcal{S}_{0}, \\
\bar{\varphi}_{j}^{\mathcal{S}_{0}}(\cdot) & =\bar{K}_{j}(0, \cdot)+c_{j} & & \text { on } \partial \mathcal{S}_{0}, \\
\bar{\varphi}_{j}^{\mathcal{S}_{0}}(x) & \rightarrow 0 & & \text { as }|x| \rightarrow+\infty,
\end{aligned}
$$

where the constant $c_{\mathcal{S}_{0}, j}$ is such that

$$
\int_{\partial \mathcal{S}_{0}} \frac{\partial \bar{\varphi}_{j}^{\mathcal{S}_{0}}}{\partial n} \mathrm{~d} s=0 .
$$

The existence and uniqueness of such a constant $c_{\mathcal{S}_{0}, j}$ is provided by a similar argument as for 8.85)-(8.86). Proceeding as in the proof of Lemma 8.12

$$
\nabla \varphi_{j}^{\mathcal{S}_{0}}=\nabla^{\perp} \bar{\varphi}_{j}^{\mathcal{S}_{0}}
$$

where the functions $\varphi_{j}^{\mathcal{S}_{0}}$, for $j=1,2,3$, are the Kirchhoff potentials in $\mathbb{R}^{2} \backslash \mathcal{S}_{0}$ defined in (4.3). As before we introduce the vector notation for the functions $\bar{\varphi}_{j}^{\mathcal{S}_{0}}$ :

$$
\bar{\varphi}^{\mathcal{S}_{0}}:=\left(\bar{\varphi}_{1}^{\mathcal{S}_{0}}, \bar{\varphi}_{2}^{\mathcal{S}_{0}}, \bar{\varphi}_{3}^{\mathcal{S}_{0}}\right)
$$

Then, following the strategy of Proposition 8.4 we obtain the following result. 
Proposition 8.13. There exist

(i) $\boldsymbol{p}_{r}^{\partial \mathcal{S}_{0}}: \mathfrak{Q} \rightarrow L^{2}\left(\partial \mathcal{S}_{0} ; \mathbb{R}^{3}\right)$ such that for any $(\varepsilon, q)$ in $\mathfrak{Q}$, with $q=(\vartheta, h)$, for any $X$ in $\partial \mathcal{S}_{0}$,

$$
\frac{\partial \overline{\boldsymbol{\varphi}}_{\varepsilon}}{\partial n}(q, \varepsilon R(\vartheta) X+h)=I_{\varepsilon}\left(\frac{\partial \bar{\varphi}^{\mathcal{S}_{0}}}{\partial n}(X)+\varepsilon^{2} \boldsymbol{p}_{r}^{\partial \mathcal{S}_{0}}(\varepsilon, q, X)\right),
$$

(ii) $\boldsymbol{p}_{r}^{\partial \Omega}: \mathfrak{Q} \rightarrow L^{2}\left(\partial \Omega ; \mathbb{R}^{3}\right)$ such that for any $(\varepsilon, q)$ in $\mathfrak{Q}$, for any $x$ in $\partial \Omega$,

$$
\frac{\partial \overline{\boldsymbol{\varphi}}_{\varepsilon}}{\partial n}(q, x)=I_{\varepsilon} \varepsilon^{2} \boldsymbol{p}_{r}^{\partial \Omega}(\varepsilon, q, x) .
$$

and moreover such that for any $\delta>0$, there exists $\varepsilon_{0}$ in $(0,1)$ for which $\boldsymbol{p}_{r}^{\partial \mathcal{S}_{0}} \in L^{\infty}\left(\mathfrak{Q}_{\delta, \varepsilon_{0}} ; L^{2}\left(\partial \mathcal{S}_{0} ; \mathbb{R}^{3}\right)\right)$ and $\boldsymbol{p}_{r}^{\partial \Omega} \in L^{\infty}\left(\mathfrak{Q}_{\delta, \varepsilon_{0}} ; L^{2}\left(\partial \Omega ; \mathbb{R}^{3}\right)\right)$.

Proposition 8.5then follows from Lemma 8.12, 8.94) and Proposition 8.13. We omit the details.

\section{Proof of the normal forms}

This section is devoted to the proof of the normal forms in Proposition 6.3 Proposition 7.10 and Proposition 7.18, as well as the expansion of the added inertia in Proposition 9.1 that was used to establish Lemma 6.5. The proof of the normal forms (6.7), (7.10) and (7.18) consists first in expanding the functions $M_{\varepsilon}(q),\left\langle\Gamma_{\varepsilon}(q), p, p\right\rangle$ and $F_{\varepsilon}(q, p)$ with respect to $\varepsilon$ thanks to the expansions of the previous sections and to Lamb's lemma (Lemma 4.6), and then in substituting these expansions into 6.1). Next, further modifications are needed in order to reach the exact forms (6.7), 7.10) and (7.18).

9.1 Asymptotic expansion of the added inertia and the Christoffel symbols

In this subsection, we use the asymptotic developments of Section 8 to deduce expansions for the added inertia matrix and for the Christoffel symbols.

We begin by giving the expansions in terms of $\varepsilon$ of the inertia matrix $M_{a, \varepsilon}(q)$ which is the counterpart for the body of size $\varepsilon$ of the added mass $M_{a}(q)$ defined in 2.16b. Precisely, it is defined for $(\varepsilon, q)$ in $\mathfrak{Q}$ by

$$
M_{a, \varepsilon}(q):=\int_{\partial \mathcal{S}_{\varepsilon}(q)} \boldsymbol{\varphi}_{\varepsilon}(q, \cdot) \otimes \frac{\partial \boldsymbol{\varphi}_{\varepsilon}}{\partial n}(q, \cdot) \mathrm{d} s=\int_{\partial \mathcal{S}_{\varepsilon}(q)} \boldsymbol{\varphi}_{\varepsilon}(q, \cdot) \otimes \boldsymbol{K}_{\varepsilon}(q, \cdot) \mathrm{d} s .
$$

The function $\varphi_{\varepsilon}$ mentioned above is defined in 8.29), 8.30). Let us also recall that the matrix $M_{a, \vartheta}^{\mathcal{S}_{0}}$ is defined in 4.9 ) and 4.10), and $I_{\varepsilon}$ is defined in 4.28). The expansion is as follows.

Proposition 9.1. There exists a function $M_{r}: \mathfrak{Q} \rightarrow \mathbb{R}^{3 \times 3}$ depending on $\mathcal{S}_{0}$ and $\Omega$ such that for any $\delta>0$ there exists $\varepsilon_{0}$ in $(0,1)$ such that $M_{r} \in$ $L^{\infty}\left(\mathfrak{Q}_{\delta, \varepsilon_{0}} ; \mathbb{R}^{3 \times 3}\right)$ and such that for all $(\varepsilon, q)$ in $\mathfrak{Q}$, with $q=(\vartheta, h)$,

$$
M_{a, \varepsilon}(q)=\varepsilon^{2} I_{\varepsilon}\left(M_{a, \vartheta}^{\mathcal{S}_{0}}+\varepsilon^{2} M_{r}(\varepsilon, q)\right) I_{\varepsilon} .
$$


Proof Using a change of variable, 2.10 and 8.30 we deduce that for $(\varepsilon, q)$ in $\mathfrak{Q}$,

$$
\boldsymbol{K}_{\varepsilon}(q, \varepsilon R(\vartheta) \cdot+h)=I_{\varepsilon} \mathcal{R}(\vartheta) \boldsymbol{K}(0, \cdot) \text { on } \partial \mathcal{S}_{0} .
$$

It follows that

$$
M_{a, \varepsilon}(q)=\varepsilon \int_{\partial \mathcal{S}_{0}} \boldsymbol{\varphi}_{\varepsilon}(q, \varepsilon R(\vartheta) \cdot+h) \otimes I_{\varepsilon} \mathcal{R}(\vartheta) \boldsymbol{K}(0, \cdot) \mathrm{d} s .
$$

We now apply Proposition 8.5. (i) to get

$$
\begin{aligned}
M_{a, \varepsilon}(q) & =\varepsilon^{2} I_{\varepsilon}\left(\int_{\partial \mathcal{S}_{0}} \mathcal{R}(\vartheta)\left(\varphi^{\mathcal{S}_{0}}+\check{\boldsymbol{c}}(\varepsilon, q)+\varepsilon^{2} \boldsymbol{\varphi}_{r}(\varepsilon, q, \cdot)\right) \otimes \mathcal{R}(\vartheta) \boldsymbol{K}(0, \cdot) \mathrm{d} s\right) I_{\varepsilon} \\
& =\varepsilon^{2} I_{\varepsilon}\left(M_{a, \vartheta}^{\mathcal{S}_{0}}+\varepsilon^{2} \mathcal{R}(\vartheta) \int_{\partial \mathcal{S}_{0}} \boldsymbol{\varphi}_{r}(\varepsilon, q, \cdot) \otimes \boldsymbol{K}(0, \cdot) \mathrm{d} s \mathcal{R}(\vartheta)^{t}\right) I_{\varepsilon},
\end{aligned}
$$

since

and

$$
\int_{\partial \mathcal{S}_{0}} \check{\boldsymbol{c}}(\varepsilon, q) \otimes \boldsymbol{K}(0, \cdot) \mathrm{d} s=\check{\boldsymbol{c}}(\varepsilon, q) \otimes \int_{\partial \mathcal{S}_{0}} \boldsymbol{K}(0, \cdot) \mathrm{d} s=0
$$

$$
M_{a, \vartheta}^{\mathcal{S}_{0}}=\mathcal{R}(\vartheta) \int_{\partial \mathcal{S}_{0}} \varphi^{\mathcal{S}_{0}} \otimes \boldsymbol{K}(0, \cdot) \mathrm{d} s \mathcal{R}(\vartheta)^{t},
$$

thanks to $4.3 \mathrm{~b}, 4.9$ and 4.10 . Above $\varepsilon_{0}$ belongs to $(0,1)$ and $\boldsymbol{\varphi}_{r}$ is in the space $L^{\infty}\left(\mathfrak{Q}_{\delta, \varepsilon_{0}} ; L^{2}\left(\partial \mathcal{S}_{0} ; \mathbb{R}^{3}\right)\right)$. Then we set

$$
M_{r}(\varepsilon, q):=\mathcal{R}(\vartheta) \int_{\partial \mathcal{S}_{0}} \boldsymbol{\varphi}_{r}(\varepsilon, q, \cdot) \otimes \boldsymbol{K}(0, \cdot) \mathrm{d} s \mathcal{R}(\vartheta)^{t},
$$

and we observe that $M_{r}$ is in $L^{\infty}\left(\mathfrak{Q}_{\delta, \varepsilon_{0}} ; \mathbb{R}\right)$ and depends only on $\mathcal{S}_{0}$ and $\Omega$. This concludes the proof of Proposition 9.1 and consequently of Lemma 6.5

We now consider the Christoffel symbols $\Gamma_{\varepsilon}^{\text {rot }}$ given for $(\varepsilon, q)$ in $\mathfrak{Q}$ and $p=(\omega, \ell) \in \mathbb{R}^{3}$, by

$$
\left\langle\Gamma_{\varepsilon}^{\mathrm{rot}}(q), p, p\right\rangle:=-\left(\begin{array}{c}
0 \\
P_{a}^{\varepsilon}
\end{array}\right) \times p-\omega M_{a, \varepsilon}(q)\left(\begin{array}{c}
0 \\
\ell^{\perp}
\end{array}\right) \in \mathbb{R}^{3},
$$

where $P_{a}^{\varepsilon}$ denotes the last two coordinates of $M_{a, \varepsilon}(q) p$. The formula $(9.3)$ is the counterpart for a body of size $\varepsilon$ of the Christoffel symbols given by $(2.26)$ when $\varepsilon=1$.

The next result proves that the leading term of $\Gamma_{\varepsilon}^{\text {rot }}$ is given, up to an appropriate scaling, by the Christoffel symbols $\left\langle\Gamma_{\vartheta}^{\mathcal{S}_{0}}, p, p\right\rangle$ of the solid as if it was immersed in a fluid filling the plane. We recall that $\left\langle\Gamma_{\vartheta}^{\mathcal{S}_{0}}, p, p\right\rangle$ is defined in 4.12. Precisely, we have the following result.

Proposition 9.2. There exists $\Gamma_{r}^{\text {rot }}: \mathfrak{Q} \rightarrow \mathcal{B L}\left(\mathbb{R}^{3} \times \mathbb{R}^{3} ; \mathbb{R}^{3}\right)$ depending on $\mathcal{S}_{0}, \gamma$ and $\Omega$, such that for any $\delta>0$, there exists $\varepsilon_{0}$ in $(0,1)$ for which $\Gamma_{r}^{\text {rot }} \in L^{\infty}\left(\mathfrak{Q}_{\delta, \varepsilon_{0}} ; \mathcal{B L}\left(\mathbb{R}^{3} \times \mathbb{R}^{3} ; \mathbb{R}^{3}\right)\right)$ and such that for any $(\varepsilon, q)$ in $\mathfrak{Q}$, with $q=(\vartheta, h)$, for any $p=(\omega, \ell)$ in $\mathbb{R}^{3}$,

$$
\left\langle\Gamma_{\varepsilon}^{\mathrm{rot}}(q), p, p\right\rangle=\varepsilon I_{\varepsilon}\left(\left\langle\Gamma_{\vartheta}^{\mathcal{S}_{0}}, I_{\varepsilon} p, I_{\varepsilon} p\right\rangle+\varepsilon^{2}\left\langle\Gamma_{r}^{\mathrm{rot}}(\varepsilon, q), I_{\varepsilon} p, I_{\varepsilon} p\right\rangle\right) .
$$


Proposition 9.2 follows from Proposition 9.1 by straightforward computations.

Finally we study the Christoffel symbols $\Gamma_{\varepsilon}^{\Omega}$ given for $(\varepsilon, q)$ in $\mathfrak{Q}$ and $p=\left(p_{1}, p_{2}, p_{3}\right) \in \mathbb{R}^{3}$, by

$$
\left\langle\Gamma_{\varepsilon}^{\partial \Omega}(q), p, p\right\rangle:=\left(\sum_{1 \leqslant k, l \leqslant 3}\left(\Gamma_{\varepsilon}^{\partial \Omega}\right)_{k, l}^{j}(q) p_{k} p_{l}\right)_{1 \leqslant j \leqslant 3} \in \mathbb{R}^{3}
$$

where for every $j, k, l \in\{1,2,3\}$, we set

$$
\left(\Gamma_{\varepsilon}^{\partial \Omega}\right)_{k, l}^{j}(q):=\frac{1}{2}\left[\Lambda_{\varepsilon, k j}^{l}(q)+\Lambda_{\varepsilon, j l}^{k}(q)-\Lambda_{\varepsilon, k l}^{j}(q)\right],
$$

with

$$
\Lambda_{\varepsilon, k j}^{l}(q):=\int_{\partial \Omega}\left(\frac{\partial \varphi_{j, \varepsilon}}{\partial \tau} \frac{\partial \varphi_{k, \varepsilon}}{\partial \tau} K_{l}\right)(q, \cdot) \mathrm{d} s
$$

The Christoffel symbols $\Gamma_{\varepsilon}^{\Omega}$ are the counterpart $\Gamma^{\Omega}$ defined in $(2.27)$. They are expanded as follows.

Proposition 9.3. There exists $\Gamma_{r}^{\partial \Omega}: \mathfrak{Q} \rightarrow \mathcal{B L}\left(\mathbb{R}^{3} \times \mathbb{R}^{3} ; \mathbb{R}^{3}\right)$ depending on $\mathcal{S}_{0}, \gamma$ and $\Omega$, such that for any $\delta>0$, there exists $\varepsilon_{0}$ in $(0,1)$ for which $\Gamma_{r}^{\partial \Omega} \in L^{\infty}\left(\mathfrak{Q}_{\delta, \varepsilon_{0}} ; \mathcal{B L}\left(\mathbb{R}^{3} \times \mathbb{R}^{3} ; \mathbb{R}^{3}\right)\right)$ and such that for any $(\varepsilon, q)$ in $\mathfrak{Q}$, with $q:=(\vartheta, h)$, for any $p:=(\omega, \ell)$ in $\mathbb{R}^{3}$,

$$
\left\langle\Gamma_{\varepsilon}^{\partial \Omega}(q), p, p\right\rangle=\varepsilon^{3} I_{\varepsilon}\left\langle\Gamma_{r}^{\partial \Omega}(\varepsilon, q), I_{\varepsilon} p, I_{\varepsilon} p\right\rangle
$$

Proof Proposition 9.3 follows from Proposition 8.5. (iii). Indeed, 9.5 can be rewritten as:

$$
\left\langle\Gamma_{\varepsilon}^{\partial \Omega}(q), p, p\right\rangle=\int_{\partial \Omega}\left[\frac{\partial \boldsymbol{\varphi}_{\varepsilon}}{\partial \tau}\left(\mathbf{K}_{\varepsilon} \cdot p\right)\left(\frac{\partial \boldsymbol{\varphi}_{\varepsilon}}{\partial \tau} \cdot p\right)-\frac{1}{2} \mathbf{K}_{\varepsilon}\left(\frac{\partial \boldsymbol{\varphi}_{\varepsilon}}{\partial \tau} \cdot p\right)^{2}\right](q, \cdot) \mathrm{d} s
$$

Observe that $\mathbf{K}_{\varepsilon}$ is actually independent of $\varepsilon$ on $\partial \Omega$. According to 8.33 , we obtain:

$$
\begin{aligned}
& \left\langle\Gamma_{\varepsilon}^{\partial \Omega}(q), p, p\right\rangle \\
& \quad=\varepsilon^{3} I_{\varepsilon} \int_{\partial \Omega}\left[\mathrm{p}_{r}^{\partial \Omega}\left(\hat{\mathbf{K}}_{\varepsilon} \cdot I_{\varepsilon} p\right)\left(\mathrm{p}_{r}^{\partial \Omega} \cdot I_{\varepsilon} p\right)-\frac{1}{2} \hat{\mathbf{K}}_{\varepsilon}\left(\mathrm{p}_{r}^{\partial \Omega} \cdot I_{\varepsilon} p\right)^{2}\right](q, \cdot) \mathrm{d} s
\end{aligned}
$$

where $\hat{\mathbf{K}}_{\varepsilon}:=\varepsilon I_{\varepsilon}^{-1} \mathbf{K}_{\varepsilon}$. This gives the expected result. 
9.2 Asymptotic expansion of $E_{\varepsilon}$

We now consider the expansion of $E_{\varepsilon}$ which is given, for $(\varepsilon, q)$ in $\mathfrak{Q}$, by

$$
E_{\varepsilon}(q):=-\frac{1}{2} \int_{\partial \mathcal{S}_{\varepsilon}(q)}\left|\frac{\partial \psi_{\varepsilon}}{\partial n}(q, \cdot)\right|^{2} \boldsymbol{K}_{\varepsilon}(q, \cdot) \mathrm{d} s .
$$

This formula is the counterpart of $(2.18 \mathrm{~b}$ for a body of size $\varepsilon$. We recall that the function $\psi_{\varepsilon}(q, \cdot)$ is defined in (8.14) and the vector field $\boldsymbol{K}_{\varepsilon}(q, \cdot)$ in 8.28 - 8.30.

The first two terms in the asymptotic expansion will be given respectively thanks to two vector fields $\mathrm{E}_{0}(q)$ and $\mathrm{E}_{1}(q)$ which we now define. First we set

$$
\mathrm{E}_{0}(q):=-\left(\begin{array}{c}
u^{\Omega}(h) \cdot \zeta_{\vartheta} \\
u^{\Omega}(h)^{\perp}
\end{array}\right) \text {, where } q=(\vartheta, h) .
$$

We recall that $u^{\Omega}$ and $\zeta_{\vartheta}$ were defined in 2.44 and 4.15-4.13 respectively. Next we define $\mathbf{E}_{1}(q)$ as

$$
\mathrm{E}_{1}(q):=\mathrm{E}_{1}^{a}(q)+\mathrm{E}_{1}^{b}(q)+\mathrm{E}_{1}^{c}(q),
$$

where the three addends are given by the definitions below.

The inertial subprincipal term $\mathrm{E}_{1}^{a}$. The definition of the term $\mathrm{E}_{1}^{a}$ will use some functions of the entries of the matrix $M_{a}^{\mathcal{S}_{0}}$ defined in 4.9$)$. Let us first recall that we decomposed $M_{a}^{\mathcal{S}_{0}}$ in 4.25 . We also define the real traceless symmetric $2 \times 2$ matrix $M^{\dagger}$ defined by

$$
M^{\dagger}=\left(M_{i, j}^{\dagger}\right)_{1 \leqslant i, j \leqslant 2}:=\frac{1}{2}\left(M_{b}^{\mathcal{S}_{0}}(\perp)+\left(M_{b}^{\mathcal{S}_{0}}(\perp)\right)^{t}\right)=\frac{1}{2}\left(M_{b}^{\mathcal{S}_{0}}(\perp)-(\perp) M_{b}^{\mathcal{S}_{0}}\right),
$$

where $(\perp)$ is defined as the $2 \times 2$ matrix

$$
(\perp):=\left(\begin{array}{cc}
0 & -1 \\
1 & 0
\end{array}\right)
$$

The matrix $M^{\dagger}$ depends only on $\mathcal{S}_{0}$. Its coefficients can be described as follows:

$$
\begin{array}{r}
M_{1,1}^{\dagger}=-M_{2,2}^{\dagger}=\int_{\partial \mathcal{S}_{0}} \frac{\partial \varphi_{3}^{\mathcal{S}_{0}}}{\partial n} \varphi_{2}^{\mathcal{S}_{0}} \mathrm{~d} s, \quad \text { and } \\
M_{1,2}^{\dagger}=M_{2,1}^{\dagger}=\frac{1}{2} \int_{\partial \mathcal{S}_{0}}\left(\frac{\partial \varphi_{3}^{\mathcal{S}_{0}}}{\partial n} \varphi_{3}^{\mathcal{S}_{0}}-\frac{\partial \varphi_{2}^{\mathcal{S}_{0}}}{\partial n} \varphi_{2}^{\mathcal{S}_{0}}\right) \mathrm{d} s
\end{array}
$$

where the functions $\varphi_{j}^{\mathcal{S}_{0}}$, for $j=1,2,3$, are the Kirchhoff potentials in $\mathbb{R}^{2} \backslash \mathcal{S}_{0}$ defined in 4.3). Recalling 4.25, we also consider

$$
M_{b, \vartheta}^{\mathcal{S}_{0}}:=R(\vartheta) M_{b}^{\mathcal{S}_{0}} R(\vartheta)^{t}, \quad \mu_{\vartheta}^{\mathcal{S}_{0}}:=R(\vartheta) \mu^{\mathcal{S}_{0}}, \quad M_{\vartheta}^{\dagger}:=R(\vartheta) M^{\dagger} R(\vartheta)^{t} .
$$


Then we define:

$$
\mathrm{E}_{1}^{a}(q):=\left(\begin{array}{c}
u^{\Omega}(h)^{\perp} M_{\vartheta}^{\dagger} u^{\Omega}(h)^{\perp} \\
0 \\
0
\end{array}\right), \text { where } q=(\vartheta, h) .
$$

The weakly gyroscopic subprincipal term $\mathrm{E}_{1}^{b}$. Let us introduce the geometrical constant $2 \times 2$ matrix

$$
\begin{aligned}
\sigma & :=\int_{\partial \mathcal{S}_{0}} \frac{\partial \psi_{-1}^{\mathcal{S}_{0}}}{\partial n}(X) X \otimes X^{\perp} \mathrm{d} s(X)+\zeta \otimes \zeta^{\perp} \\
& =\int_{\partial \mathcal{S}_{0}} \frac{\partial \psi_{-1}^{\mathcal{S}_{0}}}{\partial n}(X)\left(X \otimes X^{\perp}-\zeta \otimes \zeta^{\perp}\right) \mathrm{d} s(X),
\end{aligned}
$$

which only depends on $\mathcal{S}_{0}$. Next we introduce its symmetric part

$$
\sigma^{s}:=\frac{1}{2}\left(\sigma+\sigma^{t}\right),
$$

and the associated field force $\mathrm{E}_{1}^{b}(q)$ defined, for $q=(\vartheta, h)$ in $\mathbb{R} \times \Omega$, by

$$
\mathrm{E}_{1}^{b}(q):=\left(\begin{array}{c}
-\left\langle D_{x}^{2} \psi_{0}^{\Omega}(h, h), R(-2 \vartheta) \sigma^{s}\right\rangle_{\mathbb{R}^{2 \times 2}} \\
0 \\
0
\end{array}\right) .
$$

Let us recall that the function $\psi_{0}^{\Omega}$ is defined in 2.41). The main property of $\mathrm{E}_{1}^{b}$ is the following.

Lemma 9.4. The vector field $\mathrm{E}_{1}^{b}$ in $C^{\infty}\left(\mathbb{R} \times \Omega ; \mathbb{R}^{3}\right)$ defined by 9.15 is weakly gyroscopic in the sense of Definition 7.9.

Proof Multiply 9.15 by $\tilde{p}$ and integrate. The conclusion follows from an integration by parts, crude bounds, Lemma 6.1. the smoothness of the function $\psi_{0}^{\Omega}$ and Lemma 7.8

The drift subprincipal term $\mathrm{E}_{1}^{c}$. Let us introduce the force field $\mathrm{E}_{1}^{c}(q)$ defined, for $q=(\vartheta, h)$ in $\mathbb{R} \times \Omega$, by

$$
\mathrm{E}_{1}^{c}(q):=-\left(\begin{array}{c}
\zeta_{\vartheta} \cdot u_{c}(q) \\
\left(u_{c}(q)\right)^{\perp}
\end{array}\right) .
$$

Above $u_{c}(q)$ denotes the corrector velocity defined in 7.7 .

Now the goal of this subsection is to establish the following result.

Proposition 9.5. There exists a function $E_{r}: \mathfrak{Q} \rightarrow \mathbb{R}^{3}$ depending on $\mathcal{S}_{0}$ and $\Omega$, such that for any $\delta>0$, there exists $\varepsilon_{0}$ in $(0,1)$ for which $E_{r}$ belongs to $L^{\infty}\left(\mathfrak{Q}_{\delta, \varepsilon_{0}} ; \mathbb{R}^{3}\right)$ and such that for any $(\varepsilon, q)$ in $\mathfrak{Q}_{\delta, \varepsilon_{0}}$,

$$
E_{\varepsilon}(q)=I_{\varepsilon}\left(\mathrm{E}_{0}(q)+\varepsilon \mathrm{E}_{1}(q)+\varepsilon^{2} E_{r}(\varepsilon, q)\right) .
$$


Proof We proceed in three steps: first we use a change of variable in order to recast $E_{\varepsilon}(q)$ as an integral on the fixed boundary $\partial \mathcal{S}_{0}$. Then we plug the expansion of $\psi^{\varepsilon}$ into this integral. Finally we use several times Lamb's lemma in order to compute the terms of the resulting expansion.

First, thanks to a change of variable and $(9.2$,

$$
E_{\varepsilon}(q)=-\frac{\varepsilon}{2} I_{\varepsilon} \mathcal{R}(\vartheta) \int_{\partial \mathcal{S}_{0}}\left|\frac{\partial \psi_{\varepsilon}}{\partial n}(q, \varepsilon R(\vartheta) \cdot+h)\right|^{2} \boldsymbol{K}(0, \cdot) \mathrm{d} s,
$$

where $\boldsymbol{K}(q, \cdot)$ is the vector field defined in 2.10 . Now let $\delta>0$. Using Proposition 8.4 we deduce that there exists $\varepsilon_{0}$ in $(0,1)$ such that for any $(\varepsilon, q)$ in $\mathfrak{Q}_{\delta, \varepsilon_{0}}$,

$$
E_{\varepsilon}(q)=I_{\varepsilon} \mathcal{R}(\vartheta)\left(\frac{1}{\varepsilon} \underline{\mathrm{E}}_{-1}+\underline{\mathrm{E}}_{o}(q)+\varepsilon \underline{\mathrm{E}}_{1}(q)+\varepsilon^{2} \underline{E}_{r}(\varepsilon, q)\right),
$$

with

$$
\begin{aligned}
\underline{\mathrm{E}}_{-1} & :=-\frac{1}{2} \int_{\partial \mathcal{S}_{0}}\left|\frac{\partial \psi_{-1}^{\mathcal{S}_{0}}}{\partial n}\right|^{2} \boldsymbol{K}(0, \cdot) \mathrm{d} s \\
\underline{\mathrm{E}}_{0}(q) & :=-\int_{\partial \mathcal{S}_{0}} \frac{\partial \psi_{-1}^{\mathcal{S}_{0}}}{\partial n}\left(\frac{\partial \psi_{0}^{\mathcal{S}_{0}}}{\partial n}(q, \cdot)-R(\vartheta)^{t} u^{\Omega}(h) \cdot \tau\right) \boldsymbol{K}(0, \cdot) \mathrm{d} s, \\
\underline{\mathrm{E}}_{1}(q) & :=\underline{\mathrm{E}}_{1}^{a}(q)+\underline{\mathrm{E}}_{1}^{b}(q)
\end{aligned}
$$

where

$$
\begin{aligned}
& \underline{\mathrm{E}}_{1}^{a}(q):=-\frac{1}{2} \int_{\partial \mathcal{S}_{0}}\left|\frac{\partial \psi_{0}^{\mathcal{S}_{0}}}{\partial n}(q, \cdot)-R(\vartheta)^{t} u^{\Omega}(h) \cdot \tau\right|^{2} \boldsymbol{K}(0, \cdot) \mathrm{d} s, \\
& \underline{\mathrm{E}}_{1}^{b}(q):=-\int_{\partial \mathcal{S}_{0}} \frac{\partial \psi_{-1}^{\mathcal{S}_{0}}}{\partial n}\left(\frac{\partial \psi_{1}^{\mathcal{S}_{0}}}{\partial n}(q, \cdot)-\frac{\partial P_{1}}{\partial n}(q, \cdot)\right) \boldsymbol{K}(0, \cdot) \mathrm{d} s,
\end{aligned}
$$

and $\underline{E}_{r}$ in $L^{\infty}\left(\mathfrak{Q}_{\delta, \varepsilon_{0}} ; \mathbb{R}^{3}\right)$ depending only on $\mathcal{S}_{0}$ and $\Omega$.

We now compute each term thanks to Lamb's lemma. More precisely we establish the following equalities:

$$
\begin{aligned}
\underline{\mathrm{E}}_{-1} & =0, \\
\mathcal{R}(\vartheta) \underline{\mathrm{E}}_{0}(q) & =\mathrm{E}_{0}(q), \\
\mathcal{R}(\vartheta) \underline{\mathrm{E}}_{1}^{a}(q) & =\mathrm{E}_{1}^{a}(q), \\
\mathcal{R}(\vartheta) \underline{\mathrm{E}}_{1}^{b}(q) & =\mathrm{E}_{1}^{b}(q)+\mathrm{E}_{1}^{c}(q) .
\end{aligned}
$$

The proof of Proposition 9.5 is then concluded after observing that $\mathcal{R}(\vartheta) \underline{E}_{r}$ is also in $L^{\infty}\left(\mathfrak{Q}_{\delta, \varepsilon_{0}} ; \mathbb{R}^{3}\right)$ and depends only on $\mathcal{S}_{0}$ and $\Omega$.

To simplify the notations we omit to write the dependence on $q$ except if this dependence reduces on $\vartheta$ or $h$. Similarly we omit to write that the function 
$\boldsymbol{K}$, its coordinates $K_{j}$ and the vector fields $\xi_{j}$, which appear thanks to Lamb's lemma, are evaluated at $q=0$.

Proof of 9.23). Computation of $\underline{\mathrm{E}}_{-1}$. We use Lemma 4.6 with $u=v=$ $\nabla^{\perp} \psi_{-1}^{\mathcal{S}_{0}}$ and observe that $\nabla^{\perp} \psi_{-1}^{\mathcal{S}_{0}}$ is tangent to $\mathcal{S}_{0}$ to obtain (9.23).

Proof of 9.24). Computation of $\underline{\mathrm{E}}_{0}$. We observe that

$$
\begin{aligned}
\nabla^{\perp} \psi_{-1}^{\mathcal{S}_{0}} & =-\frac{\partial \psi_{-1}^{\mathcal{S}_{0}}}{\partial n} \tau \text { on } \partial \mathcal{S}_{0}, \\
\tau \cdot \nabla^{\perp} \psi_{0}^{\mathcal{S}_{0}} & =-\frac{\partial \psi_{0}^{\mathcal{S}_{0}}}{\partial n} \text { on } \partial \mathcal{S}_{0} .
\end{aligned}
$$

Hence for $j=1,2,3$,

$$
-\int_{\partial \mathcal{S}_{0}} \frac{\partial \psi_{-1}^{\mathcal{S}_{0}}}{\partial n} \cdot \frac{\partial \psi_{0}^{\mathcal{S}_{0}}}{\partial n} K_{j} \mathrm{~d} s=-\int_{\partial \mathcal{S}_{0}} \nabla \psi_{-1}^{\mathcal{S}_{0}} \cdot \nabla \psi_{0}^{\mathcal{S}_{0}} K_{j} \mathrm{~d} s,
$$

and we use Lemma 4.6 with $(u, v)=\left(\nabla^{\perp} \psi_{-1}^{\mathcal{S}_{0}}, \nabla^{\perp} \psi_{0}^{\mathcal{S}_{0}}\right)$ to obtain

$$
-\int_{\partial \mathcal{S}_{0}} \frac{\partial \psi_{-1}^{\mathcal{S}_{0}}}{\partial n} \cdot \frac{\partial \psi_{0}^{\mathcal{S}_{0}}}{\partial n} K_{j} \mathrm{~d} s=-\int_{\partial \mathcal{S}_{0}}\left(\xi_{j} \cdot \nabla^{\perp} \psi_{-1}^{\mathcal{S}_{0}}\right)\left(n \cdot \nabla^{\perp} \psi_{0}^{\mathcal{S}_{0}}\right) \mathrm{d} s .
$$

Then we use again (9.27) and observe that applying the tangential derivative to $8.17 \mathrm{~b}$, taking 8.15 into account, yields

$$
n \cdot \nabla^{\perp} \psi_{0}^{\mathcal{S}_{0}}=\frac{\partial \psi_{0}^{\mathcal{S}_{0}}}{\partial \tau}=R(\vartheta)^{t} u^{\Omega}(h)^{\perp} \cdot \tau \text { on } \partial \mathcal{S}_{0} .
$$

Thus

$$
-\int_{\partial \mathcal{S}_{0}} \frac{\partial \psi_{-1}^{\mathcal{S}_{0}}}{\partial n} \cdot \frac{\partial \psi_{0}^{\mathcal{S}_{0}}}{\partial n} K_{j} \mathrm{~d} s=\int_{\partial \mathcal{S}_{0}} \frac{\partial \psi_{-1}^{\mathcal{S}_{0}}}{\partial n}\left(\xi_{j} \cdot \tau\right)\left(R(\vartheta)^{t} u^{\Omega}(h)^{\perp} \cdot \tau\right) \mathrm{d} s .
$$

Then using 4.5d, 4.13) and 4.15, we arrive at

$\mathcal{R}(\vartheta) \underline{\mathrm{E}}_{0}=\mathcal{R}(\vartheta)\left(\int_{\partial \mathcal{S}_{0}} \frac{\partial \psi_{-1}^{\mathcal{S}_{0}}}{\partial n}\left(\xi_{j} \cdot R(\vartheta)^{t} u^{\Omega}(h)^{\perp}\right) \mathrm{d} s\right)_{j}=-\left(\begin{array}{c}u^{\Omega}(h) \cdot \zeta_{\vartheta} \\ u^{\Omega}(h)^{\perp}\end{array}\right)=\mathrm{E}_{0}$.

Proof of 9.25. Computation of $\underline{\mathrm{E}}_{1}^{a}$. We start with expanding the square in (9.21), to get

$\underline{\mathrm{E}}_{1}^{a}=\underline{\mathrm{E}}_{1}^{a, 1}+\int_{\partial \mathcal{S}_{0}} \frac{\partial \psi_{0}^{\mathcal{S}_{0}}}{\partial n}\left(R(\vartheta)^{t} u^{\Omega}(h) \cdot \tau\right) \boldsymbol{K} \mathrm{d} s-\frac{1}{2} \int_{\partial \mathcal{S}_{0}}\left|R(\vartheta)^{t} u^{\Omega}(h) \cdot \tau\right|^{2} \boldsymbol{K} \mathrm{d} s$,

with

$$
\underline{\mathrm{E}}_{1}^{a, 1}=-\frac{1}{2} \int_{\partial \mathcal{S}_{0}}\left|\frac{\partial \psi_{0}^{\mathcal{S}_{0}}}{\partial n}\right|^{2} \boldsymbol{K} \mathrm{d} s=-\frac{1}{2} \int_{\partial \mathcal{S}_{0}}\left|\nabla \psi_{0}^{\mathcal{S}_{0}}\right|^{2} \boldsymbol{K} \mathrm{d} s+\frac{1}{2} \int_{\partial \mathcal{S}_{0}}\left|\frac{\partial \psi_{0}^{\mathcal{S}_{0}}}{\partial \tau}\right|^{2} \boldsymbol{K} \mathrm{d} s .
$$


We apply Lemma 4.6 with $u=v=\nabla^{\perp} \psi_{0}^{\mathcal{S}_{0}}$ to get

$$
\frac{1}{2} \int_{\partial \mathcal{S}_{0}}\left|\nabla \psi_{0}^{\mathcal{S}_{0}}\right|^{2} \boldsymbol{K} \mathrm{d} s=\left(\int_{\partial \mathcal{S}_{0}}\left(\nabla^{\perp} \psi_{0}^{\mathcal{S}_{0}} \cdot n\right)\left(\nabla^{\perp} \psi_{0}^{\mathcal{S}_{0}} \cdot \xi_{j}\right) \mathrm{d} s\right)_{j=1,2,3}
$$

Let us denote by $\underline{\mathrm{E}}_{1, j}^{a, 1}, j=1,2,3$, the coordinates of the vector $\underline{\mathrm{E}}_{1}^{a, 1}$. We use 9.29) to get

$$
\begin{aligned}
\underline{\mathrm{E}}_{1, j}^{a, 1}=-\int_{\partial \mathcal{S}_{0}}\left(\xi_{j} \cdot \nabla^{\perp} \psi_{0}^{\mathcal{S}_{0}}\right)\left(R(\vartheta)^{t} u^{\Omega}(h)^{\perp} \cdot \tau\right) \mathrm{d} s & \\
& +\frac{1}{2} \int_{\partial \mathcal{S}_{0}}\left(R(\vartheta)^{t} u^{\Omega}(h)^{\perp} \cdot \tau\right)^{2} K_{j} \mathrm{~d} s .
\end{aligned}
$$

Then we decompose $\xi_{j} \cdot \nabla^{\perp} \psi_{0}^{\mathcal{S}_{0}}$ in normal and tangential parts and use again 9.29 to obtain:

$\underline{\mathrm{E}}_{1, j}^{a, 1}=\int_{\partial \mathcal{S}_{0}} \frac{\partial \psi_{0}^{\mathcal{S}_{0}}}{\partial n}\left(R(\vartheta)^{t} u^{\Omega}(h)^{\perp} \cdot \tau\right)\left(\xi_{j} \cdot \tau\right) \mathrm{d} s-\frac{1}{2} \int_{\partial \mathcal{S}_{0}}\left(R(\vartheta)^{t} u^{\Omega}(h)^{\perp} \cdot \tau\right)^{2} K_{j} \mathrm{~d} s$.

Now we plug this expression of $\underline{\mathrm{E}}_{1}^{a, 1}$ into 9.31) to get

$$
\begin{aligned}
\underline{\mathrm{E}}_{1, j}^{a}= & -\frac{1}{2} \int_{\partial \mathcal{S}_{0}}\left(R(\vartheta)^{t} u^{\Omega}(h)^{\perp}\right)^{2} K_{j} \mathrm{~d} s+\int_{\partial \mathcal{S}_{0}} \frac{\partial \psi_{0}^{\mathcal{S}_{0}}}{\partial n}\left(R(\vartheta)^{t} u^{\Omega}(h) \cdot \tau\right) K_{j} \mathrm{~d} s \\
& +\int_{\partial \mathcal{S}_{0}} \frac{\partial \psi_{0}^{\mathcal{S}_{0}}}{\partial n}\left(R(\vartheta)^{t} u^{\Omega}(h)^{\perp} \cdot \tau\right)\left(\xi_{j} \cdot \tau\right) \mathrm{d} s .
\end{aligned}
$$

We observe that the first term in the right hand side vanishes and we combine the two other ones to get

$$
\underline{\mathrm{E}}_{1, j}^{a}=\int_{\partial \mathcal{S}_{0}} \frac{\partial \psi_{0}^{\mathcal{S}_{0}}}{\partial n}\left(R(\vartheta)^{t} u^{\Omega}(h)^{\perp}\right) \cdot \xi_{j} \mathrm{~d} s .
$$

Using $8.17 \mathrm{c}$ we infer

$$
\underline{\mathbf{E}}_{1, j}^{a}=0 \text { for } j=2,3 \text {. }
$$

Now for $j=1$, we start with observing that

$$
\underline{\mathrm{E}}_{1,1}^{a}=\left(R(\vartheta)^{t} u^{\Omega}(h)^{\perp}\right) \cdot \int_{\partial \mathcal{S}_{0}} \frac{\partial \psi_{0}^{\mathcal{S}_{0}}}{\partial n} x^{\perp} \mathrm{d} s .
$$

To compute the right hand side we introduce the matrix

$$
\bar{M}:=\int_{\partial \mathcal{S}_{0}}\left(\begin{array}{c}
\bar{\varphi}_{2}^{\mathcal{S}_{0}} \\
\bar{\varphi}_{3}^{\mathcal{S}_{0}}
\end{array}\right) \otimes\left(\begin{array}{c}
\frac{\partial \bar{\varphi}_{3}^{\mathcal{S}_{0}}}{\partial n} \\
-\frac{\partial \bar{\varphi}_{2}}{\partial n}
\end{array}\right) \mathrm{d} s,
$$

where the functions $\bar{\varphi}_{j}^{\mathcal{S}_{0}}$, for $j=1,2,3$, defined in 8.93 , are harmonic conjugates to the functions $\varphi_{j}^{\mathcal{S}_{0}}$. 
Lemma 9.6. For any $q:=(\vartheta, h)$ in $\mathbb{R} \times \Omega$,

$$
\int_{\partial \mathcal{S}_{0}} \frac{\partial \psi_{0}^{\mathcal{S}_{0}}}{\partial n}(q, x) x^{\perp} \mathrm{d} s(x)=\bar{M} R(\vartheta)^{t} u^{\Omega}(h)^{\perp} .
$$

Proof First it follows from $8.93 \mathrm{~b}$ that, on $\partial \mathcal{S}_{0}$,

$$
x^{\perp}=\left(\begin{array}{c}
\bar{\varphi}_{2}^{\mathcal{S}_{0}}-c_{2}^{\mathcal{S}_{0}} \\
\bar{\varphi}_{3}^{\mathcal{S}_{0}}-c_{3}^{\mathcal{S}_{0}}
\end{array}\right)
$$

We now express the stream function $\psi_{0}^{\mathcal{S}_{0}}(q, \cdot)$ thanks to the functions $\bar{\varphi}_{3}^{\mathcal{S}_{0}}$ and $\bar{\varphi}_{2}^{\mathcal{S}_{0}}$. Let $q:=(\vartheta, h)$ in $\mathbb{R} \times \Omega$. On $\partial \mathcal{S}_{0}$, it follows from 8.15, 8.17b, 8.93 and Proposition 8.1 that there exists $c$ in $\mathbb{R}$ such that, on $\overline{\mathbb{R}^{2} \backslash \mathcal{S}_{0}}$,

$$
\psi_{0}^{\mathcal{S}_{0}}(q, \cdot)=R(\vartheta)^{t} u^{\Omega}(h)^{\perp} \cdot\left(\begin{array}{c}
\bar{\varphi}_{3}^{\mathcal{S}_{0}} \\
-\bar{\varphi}_{2}^{\mathcal{S}_{0}}
\end{array}\right)+c \psi_{-1}^{\mathcal{S}_{0}} .
$$

Then using (4.5d), 8.17c and 8.93d) we obtain $c=0$. Thus for any $q:=(\vartheta, h)$ in $\mathbb{R} \times \Omega$, on $\mathbb{R}^{2} \backslash \mathcal{S}_{0}$,

$$
\psi_{0}^{\mathcal{S}_{0}}(q, \cdot)=R(\vartheta)^{t} u^{\Omega}(h)^{\perp} \cdot\left(\begin{array}{c}
\bar{\varphi}_{3}^{\mathcal{S}_{0}} \\
-\bar{\varphi}_{2} \mathcal{S}_{0}
\end{array}\right) .
$$

Substituting (9.36) and (9.37) into the left hand side of (9.35) and using again 8.17c establishes Lemma 9.6

Then, combining $(9.33)$ and $(9.35)$, we obtain:

$$
\underline{\mathrm{E}}_{1,1}^{a}=\left(R(\vartheta)^{t} u^{\Omega}(h)^{\perp}\right) \cdot \bar{M} R(\vartheta)^{t} u^{\Omega}(h)^{\perp} .
$$

Let us now connect the matrices $M^{\dagger}$ defined in (9.8) and $\bar{M}$ defined in 9.34). Using integrations by parts and (8.94), we get, for any $i, j=2,3$,

$$
\int_{\partial \mathcal{S}_{0}} \frac{\partial \bar{\varphi}_{i}^{\mathcal{S}_{0}}}{\partial n} \bar{\varphi}_{j}^{\mathcal{S}_{0}} \mathrm{~d} s=\int_{\partial \mathcal{S}_{0}} \frac{\partial \varphi_{i}^{\mathcal{S}_{0}}}{\partial n} \varphi_{j}^{\mathcal{S}_{0}} \mathrm{~d} x
$$

Combining this with 9.10 yields

$$
M^{\dagger}=\frac{1}{2}\left(\bar{M}+\bar{M}^{t}\right) .
$$

Recalling the definition of $M_{\vartheta}^{\dagger}$ in (9.11), we deduce that

$$
\underline{\mathrm{E}}_{1,1}^{a}=u^{\Omega}(h)^{\perp} M_{\vartheta}^{\dagger} u^{\Omega}(h)^{\perp} .
$$

Gathering 9.12, 9.32 and 9.39) we obtain 9.25).

Proof of 9.26$)$. Computation of $\underline{E}_{1}^{b}$. We start by splitting $\underline{E}_{1}^{b}$ into two parts as follows:

$$
\underline{\mathrm{E}}_{1}^{b}=-\int_{\partial \mathcal{S}_{0}} \frac{\partial \psi_{-1}^{\mathcal{S}_{0}}}{\partial n} \frac{\partial \psi_{1}^{\mathcal{S}_{0}}}{\partial n} \boldsymbol{K} \mathrm{d} s+\int_{\partial \mathcal{S}_{0}} \frac{\partial \psi_{-1}^{\mathcal{S}_{0}}}{\partial n} \frac{\partial P_{1}}{\partial n} \boldsymbol{K} \mathrm{d} s
$$


Using 9.27 and 9.28 , we see that the first term of the right hand side above is equal to

$$
-\int_{\partial \mathcal{S}_{0}} \nabla^{\perp} \psi_{-1}^{\mathcal{S}_{0}} \cdot \nabla^{\perp} \psi_{1}^{\mathcal{S}_{0}} \boldsymbol{K} \mathrm{d} s
$$

We denote $\underline{\mathrm{E}}_{1, j}^{b}, j=1,2,3$, the coordinates of $\underline{\mathrm{E}}_{1}^{b}$. We apply Lemma 4.6 with $u=\nabla^{\perp} \psi_{-1}^{\mathcal{S}_{0}}$ and $v=\nabla^{\perp} \psi_{1}^{\mathcal{S}_{0}}$ for any $j=1,2,3$, to get

$$
\underline{\mathrm{E}}_{1, j}^{b}=-\int_{\partial \mathcal{S}_{0}} \frac{\partial \psi_{1}^{\mathcal{S}_{0}}}{\partial \tau} \xi_{j} \cdot \nabla^{\perp} \psi_{-1}^{\mathcal{S}_{0}} \mathrm{~d} s+\int_{\partial \mathcal{S}_{0}} \frac{\partial \psi_{-1}^{\mathcal{S}_{0}}}{\partial n} \frac{\partial P_{1}}{\partial n} K_{j} \mathrm{~d} s .
$$

We now use that, on $\partial \mathcal{S}_{0}$,

$$
\xi_{j} \cdot \nabla^{\perp} \psi_{-1}^{\mathcal{S}_{0}}=-\frac{\partial \psi_{-1}^{\mathcal{S}_{0}}}{\partial n} \xi_{j} \cdot \tau \text { and } \frac{\partial \psi_{1}^{\mathcal{S}_{0}}}{\partial \tau}=\frac{\partial P_{1}}{\partial \tau},
$$

the last identity being a consequence of $8.26 \mathrm{~b}$, to deduce that

$$
\underline{\mathrm{E}}_{1, j}^{b}=\int_{\partial \mathcal{S}_{0}} \frac{\partial \psi_{-1}^{\mathcal{S}_{0}}}{\partial n} \xi_{j} \cdot \nabla P_{1} \mathrm{~d} s .
$$

Thanks to the expression of $P_{1}$ in 8.22 ,

$$
\underline{\mathrm{E}}_{1, j}^{b}=-\left\langle D_{x}^{2} \psi_{0}^{\Omega}(h, h), R(\vartheta) A_{j}^{1} R(\vartheta)^{t}\right\rangle_{\mathbb{R}^{2 \times 2}}-D_{x} \psi_{1}^{\Omega}(q, h) \cdot R(\vartheta) A_{j}^{2},
$$

where

$$
A_{j}^{1}:=\int_{\partial \mathcal{S}_{0}} \frac{\partial \psi_{-1}^{\mathcal{S}_{0}}}{\partial n} x \otimes \xi_{j} \mathrm{~d} s \text { and } A_{j}^{2}:=\int_{\partial \mathcal{S}_{0}} \frac{\partial \psi_{-1}^{\mathcal{S}_{0}}}{\partial n} \xi_{j} \mathrm{~d} s .
$$

Case $j=1$. Consider the first term in the right hand side of (9.41). Using 9.13 we see that $A_{1}^{1}=\sigma-\zeta \otimes \zeta^{\perp}$ and we observe that, since $D_{x}^{2} \psi_{0}^{S}(h, h)$ is symmetric,

$$
\left\langle D_{x}^{2} \psi_{0}^{\Omega}(h, h), R(\vartheta) \sigma R(\vartheta)^{t}\right\rangle_{\mathbb{R}^{2 \times 2}}=\left\langle D_{x}^{2} \psi_{0}^{\Omega}(h, h), R(\vartheta) \sigma^{s} R(\vartheta)^{t}\right\rangle,
$$

where $\sigma^{s}$ is the symmetric part of $\sigma$ defined in $\left(9.14\right.$. Then using that $\sigma^{s}$ is a traceless symmetric $2 \times 2$ matrix,

$$
\left\langle D_{x}^{2} \psi_{O}^{\Omega}(h, h), R(\vartheta) \sigma R(\vartheta)^{t}\right\rangle_{\mathbb{R}^{2 \times 2}}=\left\langle D_{x}^{2} \psi_{O}^{\Omega}(h, h), R(-2 \vartheta) \sigma^{s}\right\rangle=-\mathrm{E}_{1,1}^{b}(q),
$$

where $\mathrm{E}_{1,1}^{b}(q)$ denotes the first coordinate of the vector field $\mathrm{E}_{1}^{b}(q)$ defined in 9.15). Therefore we obtain for $j=1$,

$$
-\left\langle D_{x}^{2} \psi_{0}^{\Omega}(h, h), R(\vartheta) A_{j}^{1} R(\vartheta)^{t}\right\rangle_{\mathbb{R}^{2 \times 2}}=\mathrm{E}_{1,1}^{b}(q)+\left\langle D_{x}^{2} \psi_{0}^{\Omega}(h, h), \zeta_{\vartheta} \otimes \zeta_{\vartheta}^{\perp}\right\rangle_{\mathbb{R}^{2 \times 2}} .
$$

Concerning the second term in the right hand side of 9.41 , we use $A_{1}^{2}=-\zeta^{\perp}$ (see 4.13) to get that for $j=1$,

$$
-D_{x} \psi_{1}^{\Omega}(q, h) \cdot R(\vartheta) A_{j}^{2}=D_{x} \psi_{1}^{\Omega}(q, h) \cdot \zeta_{\vartheta}^{\perp} .
$$

Thus

$$
\underline{\mathrm{E}}_{1,1}^{b}=\mathrm{E}_{1,1}^{b}(q)+\left\langle D_{x}^{2} \psi_{0}^{\Omega}(h, h), \zeta_{\vartheta} \otimes \zeta_{\vartheta}^{\perp}\right\rangle_{\mathbb{R}^{2 \times 2}}+D_{x} \psi_{1}^{\Omega}(q, h) \cdot \zeta_{\vartheta}^{\perp} .
$$

The last two terms in the right hand side can be expressed in terms of the corrector velocity $u_{c}(q)$ defined in (7.7), as follows from the following statement. 
Lemma 9.7. For any $q=(\vartheta, h)$ in $\Omega \times \mathbb{R}$,

$$
u_{c}(q)=\left(D_{x}^{2} \psi_{0}^{\Omega}(h, h) \cdot \zeta_{\vartheta}+D_{x} \psi_{1}^{\Omega}(q, h)\right)^{\perp} .
$$

Proof From the definition of $\psi_{c}$ in 6.10 and the one of $u_{c}(q)$ in 7.7 we deduce that for any $q=(\vartheta, h)$ in $\Omega \times \mathbb{R}$,

$$
u_{c}(q)=\left(D_{x}^{2} \psi_{O}^{\Omega}(h, h) \cdot \zeta_{\vartheta}+D_{x h}^{2} \psi_{O}^{\Omega}(h, h) \cdot \zeta_{\vartheta}\right)^{\perp}
$$

which yields 9.42 thanks to 8.19 .

Hence we finally obtain

$$
\underline{\mathrm{E}}_{1,1}^{b}=\mathrm{E}_{1,1}^{b}(q)-\zeta_{\vartheta} \cdot u_{c}(q) .
$$

Case $j=2$ or 3 . In this case, $A_{j}^{1}=-\zeta \otimes \xi_{j}$ and $A_{j}^{2}=-\xi_{j}$, and therefore

$$
\begin{aligned}
\underline{\mathrm{E}}_{1, j}^{b} & =\left\langle D_{x}^{2} \psi_{O}^{\Omega}(h, h), R(\vartheta)\left(\zeta \otimes \xi_{j}\right) R(\vartheta)^{t}\right\rangle_{\mathbb{R}^{2 \times 2}}+D \psi_{1}^{\Omega}(q, h) \cdot R(\vartheta) \xi_{j} \\
& =\left(D_{x}^{2} \psi_{0}^{\Omega}(h, h) \cdot \zeta_{\vartheta}+D \psi_{1}^{\Omega}(q, h)\right) \cdot R(\vartheta) \xi_{j} \\
& =-R(\vartheta)^{t} u_{c}(q)^{\perp} \cdot \xi_{j} .
\end{aligned}
$$

Thus

$$
R(\vartheta)\left(\underline{\mathrm{E}}_{1,1}^{b}\right)_{j=2,3}=-u_{c}(q)^{\perp} .
$$

Gathering 9.15, 9.16), 9.43 and 9.44) we obtain 9.26). This ends the proof of Proposition 9.5

9.3 Asymptotic expansion of $B_{\varepsilon}$

We now tackle the expansion of $B_{\varepsilon}$ which is given, for $(\varepsilon, q)$ in $\mathfrak{Q}$, by

$$
B_{\varepsilon}(q):=\int_{\partial \mathcal{S}_{\varepsilon}(q)} \frac{\partial \psi_{\varepsilon}}{\partial n}(q, \cdot)\left(\boldsymbol{K}_{\varepsilon}(q, \cdot) \times \frac{\partial \varphi_{\varepsilon}}{\partial \tau}(q, \cdot)\right) \mathrm{d} s .
$$

This formula is the counterpart of 2.18a for a body of size $\varepsilon$. Let us recall that the Kirchhoff potentials $\varphi_{\varepsilon}$ are defined in 8.29-8.30.

The expansion that we obtain for $B_{\varepsilon}(q)$ is given in the following statement where $B_{\vartheta}^{\mathcal{S}_{0}}$ is defined in 4.16), $M_{\vartheta}^{\dagger}$ in (9.11), $I_{\varepsilon}$ in 4.28) and where:

$$
\mathrm{B}_{1}(q):=\left(\begin{array}{c}
0 \\
-2 M_{\vartheta}^{\dagger} u^{\Omega}(h)^{\perp}
\end{array}\right) \text { for } q=(\vartheta, h) .
$$

Proposition 9.8. There exists $B_{r}: \mathfrak{Q} \rightarrow \mathbb{R}^{3}$ depending only on $\mathcal{S}_{0}$ and $\Omega$, such that for any $\delta>0$, there exists $\varepsilon_{0}$ in $(0,1)$ for which $B_{r} \in L^{\infty}\left(\mathfrak{Q}_{\delta, \varepsilon_{0}} ; \mathbb{R}^{3}\right)$ and such that for any $(\varepsilon, q)$ in $\mathfrak{Q}_{\delta, \varepsilon_{0}}$, where $q=(\vartheta, h)$,

$$
B_{\varepsilon}(q)=\varepsilon I_{\varepsilon}^{-1}\left(B_{\vartheta}^{\mathcal{S}_{0}}+\varepsilon \mathrm{B}_{1}(q)+\varepsilon^{2} B_{r}(\varepsilon, q)\right) .
$$


Proof We proceed as in the proof of Proposition 9.5 Let us state the following formula which is useful several times in the sequel:

$$
\text { for any }\left(p_{a}, p_{b}\right) \in \mathbb{R}^{3} \times \mathbb{R}^{3}, \quad \varepsilon p_{a} \times p_{b}=I_{\varepsilon}\left(\left(I_{\varepsilon} p_{a}\right) \times\left(I_{\varepsilon} p_{b}\right)\right) \text {. }
$$

By a change of variable, using (9.47) and (9.2), we arrive at

$$
\begin{gathered}
B_{\varepsilon}(q)= \\
\varepsilon I_{\varepsilon}^{-1} \mathcal{R}(\vartheta) \int_{\partial \mathcal{S}_{0}} \frac{\partial \psi_{\varepsilon}}{\partial n}(q, \varepsilon R(\vartheta) \cdot+h)\left(\boldsymbol{K}(0, \cdot) \times \mathcal{R}(\vartheta)^{t} \frac{\partial \varphi_{\varepsilon}}{\partial \tau}(q, \varepsilon R(\vartheta) \cdot+h)\right) \mathrm{d} s .
\end{gathered}
$$

Now let $\delta>0$. We use Proposition 8.4 and Proposition 8.5 to obtain that there exists $\varepsilon_{0}$ in $(0,1)$ such that for any $(\varepsilon, q)$ in $\mathfrak{Q}_{\delta, \varepsilon_{0}}$,

$$
B_{\varepsilon}(q)=\varepsilon I_{\varepsilon}^{-1} \mathcal{R}(\vartheta)\left(\underline{\mathrm{B}}_{O}+\varepsilon \underline{\mathrm{B}}_{1}(q)+\varepsilon^{2} B_{r}(\varepsilon, q)\right),
$$

with

$$
\begin{aligned}
\underline{\mathrm{B}}_{0} & :=\int_{\partial \mathcal{S}_{0}} \frac{\partial \psi_{-1}^{\mathcal{S}_{0}}}{\partial n}\left(\boldsymbol{K}(0, \cdot) \times \frac{\partial \boldsymbol{\varphi}^{\mathcal{S}_{0}}}{\partial \tau}\right) \mathrm{d} s \\
\underline{\mathrm{B}}_{1}(q) & :=\int_{\partial \mathcal{S}_{0}}\left(\frac{\partial \psi_{0}^{\mathcal{S}_{0}}}{\partial n}-R(\vartheta)^{t} u^{\Omega}(h) \cdot \tau\right)\left(\boldsymbol{K}(0, \cdot) \times \frac{\partial \boldsymbol{\varphi}^{\mathcal{S}_{0}}}{\partial \tau}\right) \mathrm{d} s
\end{aligned}
$$

and $B_{r}$ in $L^{\infty}\left(\mathfrak{Q}_{\delta, \varepsilon_{0}} ; \mathbb{R}^{3}\right)$ depending only on $\mathcal{S}_{0}$ and $\Omega$.

We now compute each term thanks to Lamb's lemma. More precisely we will prove the following equalities:

$$
\begin{aligned}
& \mathcal{R}(\vartheta) \underline{\mathrm{B}}_{0}=B_{\vartheta}^{\mathcal{S}_{0}}, \\
& \mathcal{R}(\vartheta) \underline{\mathrm{B}}_{1}=\mathrm{B}_{1} .
\end{aligned}
$$

As in the proof of Proposition 9.8 we will omit to write the dependence on $q$, except if this dependence reduces to a dependence on $\vartheta$ or $h$, and it will be understood that the functions $\boldsymbol{K}$, its coordinates $K_{j}$ and the vector fields $\xi_{j}$ are evaluated at $q=0$.

Proof of 9.48). Computation of $\underline{\mathrm{B}}^{0}$. For $j=1,2,3$, we denote by $\underline{\mathrm{B}}_{0, j}$, the coordinates of $\underline{\mathrm{B}}_{O}$. By (9.27),

$$
\begin{aligned}
\underline{\mathrm{B}}_{0,1} & =\int_{\partial \mathcal{S}_{0}} \frac{\partial \psi_{-1}^{\mathcal{S}_{0}}}{\partial n} \frac{\partial \varphi_{3}^{\mathcal{S}_{0}}}{\partial \tau} K_{2} \mathrm{~d} s-\int_{\partial \mathcal{S}_{0}} \frac{\partial \psi_{-1}^{\mathcal{S}_{0}}}{\partial n} \frac{\partial \varphi_{2}^{\mathcal{S}_{0}}}{\partial \tau} K_{3} \mathrm{~d} s \\
& =-\int_{\partial \mathcal{S}_{0}} \nabla^{\perp} \psi_{-1}^{\mathcal{S}_{0}} \cdot \nabla \varphi_{3}^{\mathcal{S}_{0}} K_{2} \mathrm{~d} s+\int_{\partial \mathcal{S}_{0}} \nabla^{\perp} \psi_{-1}^{\mathcal{S}_{0}} \cdot \nabla \varphi_{2}^{\mathcal{S}_{0}} K_{3} \mathrm{~d} s
\end{aligned}
$$

Then we use Lemma 4.6 twice, with $(u, v)=\left(\nabla^{\perp} \psi_{-1}^{\mathcal{S}_{0}}, \nabla \varphi_{2}^{\mathcal{S}_{0}}\right)$ and with $(u, v)=$ $\left(\nabla^{\perp} \psi_{-1}^{\mathcal{S}_{0}}, \nabla \varphi_{3}^{\mathcal{S}_{0}}\right), 4.5 \mathrm{~d}$ ) and 9.27 to obtain

$$
\underline{\mathrm{B}}_{0,1}=\int_{\partial \mathcal{S}_{0}} \frac{\partial \psi_{-1}^{\mathcal{S}_{0}}}{\partial n}\left(\left(\tau \cdot \xi_{2}\right)\left(n \cdot \xi_{3}\right)-\left(\tau \cdot \xi_{3}\right)\left(n \cdot \xi_{2}\right)\right) \mathrm{d} s=-1 .
$$


Proceeding in the same way and using (4.13), we arrive at

$$
\underline{\mathrm{B}}_{0,2}=\int_{\partial \mathcal{S}_{0}} \frac{\partial \psi_{-1}^{\mathcal{S}_{0}}}{\partial n}\left(\left(\tau \cdot \xi_{3}\right)\left(n \cdot \xi_{1}\right)-\left(\tau \cdot \xi_{1}\right)\left(n \cdot \xi_{3}\right)\right) \mathrm{d} s=\zeta^{\perp} \cdot \xi_{2},
$$

and $\underline{\mathrm{B}}_{0,3}=\zeta^{\perp} \cdot \xi_{3}$. This gives 9.48 .

Proof of 9.49 . Computation of $\underline{B}_{1}$. Let us start with the first coordinate $\underline{\mathrm{B}}_{1,1}$ of $\underline{\mathrm{B}}_{1}$, that is:

$$
\begin{aligned}
\underline{\mathrm{B}}_{1,1} & =-\int_{\partial \mathcal{S}_{0}}\left(\frac{\partial \psi_{0}^{\mathcal{S}_{0}}}{\partial n}-R(\vartheta)^{t} u^{\Omega}(h) \cdot \tau\right)\left(\frac{\partial \varphi_{2}^{\mathcal{S}_{0}}}{\partial \tau} K_{3}-\frac{\partial \varphi_{3}^{\mathcal{S}_{0}}}{\partial \tau} K_{2}\right) \mathrm{d} s \\
& =\underline{\mathrm{B}}_{1,1}^{a}+\underline{\mathrm{B}}_{1,1}^{b}+\underline{\mathrm{B}}_{1,1}^{c},
\end{aligned}
$$

with

$$
\begin{aligned}
& \underline{\mathrm{B}}_{1,1}^{a}:=-\int_{\partial \mathcal{S}_{0}} \frac{\partial \psi_{0}^{\mathcal{S}_{0}}}{\partial n} \frac{\partial \varphi_{2}^{\mathcal{S}_{0}}}{\partial \tau} K_{3} \mathrm{~d} s, \\
& \underline{\mathrm{B}}_{1,1}^{b}:=\int_{\partial \mathcal{S}_{0}} \frac{\partial \psi_{0}^{\mathcal{S}_{0}}}{\partial n} \frac{\partial \varphi_{3}^{\mathcal{S}_{0}}}{\partial \tau} K_{2} \mathrm{~d} s, \\
& \underline{\mathrm{B}}_{1,1}^{c}:=\int_{\partial \mathcal{S}_{0}} R(\vartheta)^{t} u^{\Omega}(h) \cdot\left(\frac{\partial \varphi_{2}^{\mathcal{S}_{0}}}{\partial \tau} K_{3}-\frac{\partial \varphi_{3}^{\mathcal{S}_{0}}}{\partial \tau} K_{2}\right) \tau \mathrm{d} s .
\end{aligned}
$$

We start with

$$
\underline{\mathrm{B}}_{1,1}^{a}=\int_{\partial \mathcal{S}_{0}} \nabla^{\perp} \psi_{0}^{\mathcal{S}_{0}} \cdot \nabla \varphi_{2}^{\mathcal{S}_{0}} K_{3} \mathrm{~d} s-\int_{\partial \mathcal{S}_{0}} \frac{\partial \psi_{0}^{\mathcal{S}_{0}}}{\partial \tau} K_{2} K_{3} \mathrm{~d} s .
$$

We use Lemma 4.6 with $u=\nabla^{\perp} \psi_{0}^{\mathcal{S}_{0}}$ and $v=\nabla \varphi_{2}^{\mathcal{S}_{0}}$ to obtain

$$
\begin{aligned}
\int_{\partial \mathcal{S}_{0}} \nabla^{\perp} \psi_{0}^{\mathcal{S}_{0}} \cdot \nabla \varphi_{2}^{\mathcal{S}_{0}} K_{3} \mathrm{~d} s= & \int_{\partial \mathcal{S}_{0}}\left(\nabla^{\perp} \psi_{0}^{\mathcal{S}_{0}} \cdot \xi_{3}\right)\left(\nabla \varphi_{2}^{\mathcal{S}_{0}} \cdot n\right) \mathrm{d} s \\
& +\int_{\partial \mathcal{S}_{0}}\left(\nabla^{\perp} \psi_{0}^{\mathcal{S}_{0}} \cdot n\right)\left(\nabla \varphi_{2}^{\mathcal{S}_{0}} \cdot \xi_{3}\right) \mathrm{d} s v \\
= & \int_{\partial \mathcal{S}_{0}}\left(\frac{\partial \psi_{0}^{\mathcal{S}_{0}}}{\partial \tau} K_{3}-\frac{\partial \psi_{0}^{\mathcal{S}_{0}}}{\partial n} \xi_{3} \cdot \tau\right) K_{2} \mathrm{~d} s \\
+\int_{\partial \mathcal{S}_{0}} \frac{\partial \psi_{0}^{\mathcal{S}_{0}}}{\partial \tau} K_{2} K_{3} \mathrm{~d} s & +\int_{\partial \mathcal{S}_{0}} \frac{\partial \psi_{0}^{\mathcal{S}_{0}}}{\partial \tau} \frac{\partial \varphi_{2}^{\mathcal{S}_{0}}}{\partial \tau}\left(\xi_{3} \cdot \tau\right) \mathrm{d} s .
\end{aligned}
$$

Therefore

$$
\underline{\mathbf{B}}_{1,1}^{a}=\int_{\partial \mathcal{S}_{0}}\left(\frac{\partial \psi_{0}^{\mathcal{S}_{0}}}{\partial \tau} K_{3}-\frac{\partial \psi_{0}^{\mathcal{S}_{0}}}{\partial n} \xi_{3} \cdot \tau\right) K_{2} \mathrm{~d} s+\int_{\partial \mathcal{S}_{0}} \frac{\partial \psi_{0}^{\mathcal{S}_{0}}}{\partial \tau} \frac{\partial \varphi_{2}^{\mathcal{S}_{0}}}{\partial \tau}\left(\xi_{3} \cdot \tau\right) \mathrm{d} s
$$


By switching the indexes 2 and 3 we obtain

$$
\underline{\mathrm{B}}_{1,1}^{b}=-\int_{\partial \mathcal{S}_{0}}\left(\frac{\partial \psi_{0}^{\mathcal{S}_{0}}}{\partial \tau} K_{2}-\frac{\partial \psi_{0}^{\mathcal{S}_{0}}}{\partial n} \xi_{2} \cdot \tau\right) K_{3} \mathrm{~d} s-\int_{\partial \mathcal{S}_{0}} \frac{\partial \psi_{0}^{\mathcal{S}_{0}}}{\partial \tau} \frac{\partial \varphi_{3}^{\mathcal{S}_{0}}}{\partial \tau}\left(\xi_{2} \cdot \tau\right) \mathrm{d} s
$$

We sum these two terms, observe that $-\left(\xi_{3} \cdot \tau\right) K_{2}+\left(\xi_{2} \cdot \tau\right) K_{3}=K_{2}^{2}+K_{3}^{2}=1$ and use $8.17 \mathrm{c}$ to get

$$
\int_{\partial \mathcal{S}_{0}}\left(K_{2}^{2}+K_{3}^{2}\right) \frac{\partial \psi_{0}^{\mathcal{S}_{0}}}{\partial n} \mathrm{~d} s=\int_{\partial \mathcal{S}_{0}} \frac{\partial \psi_{0}^{\mathcal{S}_{0}}}{\partial n} \mathrm{~d} s=0
$$

We deduce

$$
\underline{\mathrm{B}}_{1,1}^{a}+\underline{\mathrm{B}}_{1,1}^{b}=\int_{\partial \mathcal{S}_{0}} \frac{\partial \psi_{0}^{\mathcal{S}_{0}}}{\partial \tau}\left(\frac{\partial \varphi_{2}^{\mathcal{S}_{0}}}{\partial \tau}\left(\xi_{3} \cdot \tau\right)-\frac{\partial \varphi_{3}^{\mathcal{S}_{0}}}{\partial \tau}\left(\xi_{2} \cdot \tau\right)\right) \mathrm{d} s .
$$

Now, using $(9.29)$, we obtain

$$
\begin{aligned}
\underline{\mathrm{B}}_{1,1}^{a}+\underline{\mathrm{B}}_{1,1}^{b} & =-\int_{\partial \mathcal{S}_{0}} R(\vartheta)^{t} u^{\Omega}(h) \cdot\left(\frac{\partial \varphi_{2}^{\mathcal{S}_{0}}}{\partial \tau}\left(\xi_{3} \cdot \tau\right)-\frac{\partial \varphi_{3}^{\mathcal{S}_{0}}}{\partial \tau}\left(\xi_{2} \cdot \tau\right)\right) n \mathrm{~d} s \\
& =R(\vartheta)^{t} u^{\Omega}(h) \cdot \int_{\partial \mathcal{S}_{0}}\left(\frac{\partial \varphi_{2}^{\mathcal{S}_{0}}}{\partial \tau}\left(\xi_{2} \cdot n\right)+\frac{\partial \varphi_{3}^{\mathcal{S}_{0}}}{\partial \tau}\left(\xi_{3} \cdot n\right)\right) n \mathrm{~d} s .
\end{aligned}
$$

On the other hand we observe that

$$
\underline{\mathrm{B}}_{1,1}^{c}=R(\vartheta)^{t} u^{\Omega}(h) \cdot \int_{\partial \mathcal{S}_{0}}\left(\frac{\partial \varphi_{2}^{\mathcal{S}_{0}}}{\partial \tau}\left(\xi_{2} \cdot \tau\right)+\frac{\partial \varphi_{3}^{\mathcal{S}_{0}}}{\partial \tau}\left(\xi_{3} \cdot \tau\right)\right) \tau \mathrm{d} s .
$$

By 9.50 and 9.51 ,

$$
\underline{\mathrm{B}}_{1,1}=R(\vartheta)^{t} u^{\Omega}(h) \cdot \int_{\partial \mathcal{S}_{0}}\left[\frac{\partial \varphi_{2}^{\mathcal{S}_{0}}}{\partial \tau} \xi_{2}+\frac{\partial \varphi_{3}^{\mathcal{S}_{0}}}{\partial \tau} \xi_{3}\right] \mathrm{d} s=0,
$$

thanks to an integration by parts.

Let us now consider the second coordinate $\underline{B}_{1,2}$ of $\underline{B}_{1}$, that is:

$$
\begin{aligned}
\underline{\mathrm{B}}_{1,2} & =\int_{\partial \mathcal{S}_{0}}\left(\frac{\partial \psi_{0}^{\mathcal{S}_{0}}}{\partial n}-R(\vartheta)^{t} u^{\Omega}(h) \cdot \tau\right)\left(\frac{\partial \varphi_{1}^{\mathcal{S}_{0}}}{\partial \tau} K_{3}-\frac{\partial \varphi_{3}^{\mathcal{S}_{0}}}{\partial \tau} K_{1}\right) \mathrm{d} s \\
& =\underline{\mathrm{B}}_{1,2}^{a}+\underline{\mathrm{B}}_{1,2}^{b}+\underline{\mathrm{B}}_{1,2}^{c},
\end{aligned}
$$

with

$$
\begin{aligned}
\underline{\mathrm{B}}_{1,2}^{a} & :=-\int_{\partial \mathcal{S}_{0}} \frac{\partial \psi_{0}^{\mathcal{S}_{0}}}{\partial n} \frac{\partial \varphi_{3}^{\mathcal{S}_{0}}}{\partial \tau} K_{1} \mathrm{~d} s \\
\underline{\mathrm{B}}_{1,2}^{b} & :=\int_{\partial \mathcal{S}_{0}} \frac{\partial \psi_{0}^{\mathcal{S}_{0}}}{\partial n} \frac{\partial \varphi_{1}^{\mathcal{S}_{0}}}{\partial \tau} K_{3} \mathrm{~d} s \\
\underline{\mathrm{B}}_{1,2}^{c} & :=\int_{\partial \mathcal{S}_{0}} R(\vartheta)^{t} u^{\Omega}(h) \cdot\left(\frac{\partial \varphi_{3}^{\mathcal{S}_{0}}}{\partial \tau} K_{1}-\frac{\partial \varphi_{1}^{\mathcal{S}_{0}}}{\partial \tau} K_{3}\right) \tau \mathrm{d} s .
\end{aligned}
$$


Proceeding as above with $\underline{\mathrm{B}}_{1,1}^{a}$ and $\underline{\mathrm{B}}_{1,1}^{b}$, we arrive at

$$
\begin{aligned}
& \underline{\mathrm{B}}_{1,2}^{a}=\int_{\partial \mathcal{S}_{0}}\left(\frac{\partial \psi_{0}^{\mathcal{S}_{0}}}{\partial \tau} K_{1}-\frac{\partial \psi_{0}^{\mathcal{S}_{0}}}{\partial n} \xi_{1} \cdot \tau\right) K_{3} \mathrm{~d} s+\int_{\partial \mathcal{S}_{0}} \frac{\partial \psi_{0}^{\mathcal{S}_{0}}}{\partial \tau} \frac{\partial \varphi_{3}^{\mathcal{S}_{0}}}{\partial \tau}\left(\xi_{1} \cdot \tau\right) \mathrm{d} s, \\
& \underline{\mathrm{B}}_{1,2}^{b}=-\int_{\partial \mathcal{S}_{0}}\left(\frac{\partial \psi_{0}^{\mathcal{S}_{0}}}{\partial \tau} K_{3}-\frac{\partial \psi_{0}^{\mathcal{S}_{0}}}{\partial n} \xi_{3} \cdot \tau\right) K_{1} \mathrm{~d} s-\int_{\partial \mathcal{S}_{0}} \frac{\partial \psi_{0}^{\mathcal{S}_{0}}}{\partial \tau} \frac{\partial \varphi_{1}^{\mathcal{S}_{0}}}{\partial \tau}\left(\xi_{3} \cdot \tau\right) \mathrm{d} s .
\end{aligned}
$$

We sum these two terms, observing that $\left(\xi_{1} \cdot \tau\right) K_{3}-\left(\xi_{3} \cdot \tau\right) K_{1}=x^{\perp} \cdot \xi_{2}$, to get

$$
\begin{aligned}
\underline{\mathrm{B}}_{1,2}^{a}+\underline{\mathrm{B}}_{1,2}^{b}=-\int_{\partial \mathcal{S}_{0}} & \left(x^{\perp} \cdot \xi_{2}\right) \frac{\partial \psi_{0}^{\mathcal{S}_{0}}}{\partial n} \mathrm{~d} s \\
& \quad+\int_{\partial \mathcal{S}_{0}} \frac{\partial \psi_{0}^{\mathcal{S}_{0}}}{\partial \tau}\left(\frac{\partial \varphi_{3}^{\mathcal{S}_{0}}}{\partial \tau}\left(\xi_{1} \cdot \tau\right)-\frac{\partial \varphi_{1}^{\mathcal{S}_{0}}}{\partial \tau}\left(\xi_{3} \cdot \tau\right)\right) \mathrm{d} s .
\end{aligned}
$$

Using (9.35) we obtain

$$
\int_{\partial \mathcal{S}_{0}}\left(x^{\perp} \cdot \xi_{2}\right) \frac{\partial \psi_{0}^{\mathcal{S}_{0}}}{\partial n} \mathrm{~d} s=\bar{M} R(\vartheta)^{t} u^{\Omega}(h)^{\perp} \cdot \xi_{2},
$$

with $\bar{M}$ given by $(9.34)$. We also use $(9.29$ to modify the second term in the right hand side of $(9.52$ and then get

$$
\begin{aligned}
\underline{\mathrm{B}}_{1,2}^{a}+\underline{\mathrm{B}}_{1,2}^{b}= & -\bar{M} R(\vartheta)^{t} u^{\Omega}(h)^{\perp} \cdot \xi_{2} \\
& -\int_{\partial \mathcal{S}_{0}} R(\vartheta)^{t} u^{\Omega}(h) \cdot\left(\frac{\partial \varphi_{3}^{\mathcal{S}_{0}}}{\partial \tau}\left(\xi_{1} \cdot \tau\right)-\frac{\partial \varphi_{1}^{\mathcal{S}_{0}}}{\partial \tau}\left(\xi_{3} \cdot \tau\right)\right) n \mathrm{~d} s .
\end{aligned}
$$

Adding $\underline{\mathrm{B}}_{1,2}^{c}$, we arrive at

$$
\underline{\mathrm{B}}_{1,2}=-\bar{M} R(\vartheta)^{t} u^{\Omega}(h)^{\perp} \cdot \xi_{2}-R(\vartheta)^{t} u^{\Omega}(h) \cdot \underline{\mathrm{B}}_{1,2}^{d},
$$

with

$$
\begin{aligned}
\underline{\mathrm{B}}_{1,2}^{d}:=\int_{\partial \mathcal{S}_{0}} \frac{\partial \varphi_{3}^{\mathcal{S}_{0}}}{\partial \tau}\left(\left(\xi_{1}^{\perp} \cdot n\right) n+\left(\xi_{1}^{\perp} \cdot \tau\right) \tau\right) \mathrm{d} s \\
\quad+\int_{\partial \mathcal{S}_{0}} \frac{\partial \varphi_{1}^{\mathcal{S}_{0}}}{\partial \tau}\left(-\left(\xi_{3}^{\perp} \cdot n\right) n-\left(\xi_{3}^{\perp} \cdot \tau\right) \tau\right) \mathrm{d} s \\
=\int_{\partial \mathcal{S}_{0}} \frac{\partial \varphi_{3}^{\mathcal{S}_{0}}}{\partial \tau} \xi_{1}^{\perp} \mathrm{d} s-\int_{\partial \mathcal{S}_{0}} \frac{\partial \varphi_{1}^{\mathcal{S}_{0}}}{\partial \tau} \xi_{3}^{\perp} \mathrm{d} s .
\end{aligned}
$$

Using an integration by parts we see that the second term of the right hand side above vanishes and the first term gives

$$
\int_{\partial \mathcal{S}_{0}} \frac{\bar{\varphi}_{3}^{\mathcal{S}_{0}}}{\partial \tau}(x) x^{\perp} \mathrm{d} s(x)=-\int_{\partial \mathcal{S}_{0}} \varphi_{3}^{\mathcal{S}_{0}} n \mathrm{~d} s=-\bar{M} \xi_{2}(0, \cdot) .
$$


Therefore

$$
R(\vartheta)^{t} u^{\Omega}(h) \cdot \underline{\mathrm{B}}_{1,2}^{d}=\bar{M}^{t} R(\vartheta)^{t} u^{\Omega}(h)^{\perp} \cdot \xi_{2} .
$$

Gathering 9.54 and 9.55 and using 9.38, we arrive at

$$
\underline{\mathrm{B}}_{1,2}=-2 M^{\dagger} R(\vartheta)^{t} u^{\Omega}(h)^{\perp} \cdot \xi_{2} .
$$

Proceeding in the same way for the third coordinate, using

$$
\left(\xi_{1} \cdot \tau\right) K_{2}-\left(\xi_{2} \cdot \tau\right) K_{1}=-x^{\perp} \cdot \xi_{3} \text { and } \int_{\partial \mathcal{S}_{0}} \frac{\partial \bar{\varphi}_{2}^{\mathcal{S}_{0}}}{\partial \tau}(x) x^{\perp} \mathrm{d} s(x)=\bar{M} \xi_{3}(0, \cdot),
$$

we deduce that

$$
\underline{\mathrm{B}}_{1,3}=-2 M^{\dagger} R(\vartheta)^{t} u^{\Omega}(h)^{\perp} \cdot \xi_{3} .
$$

Combining (9.56) and (9.57) and recalling the definition of $M_{\vartheta}^{\dagger}$ in (9.11), we deduce 9.49). This ends the proof of Proposition 9.8.

\subsection{End of the proof of the normal forms}

To prove Proposition 6.3 and Proposition 7.10 we have to put Equation 2.21 under the normal forms (6.7) and (7.10). We focus on the more delicate Case (ii). Case (i) can be proved with the same strategy with some simplifications, since the proof of the normal form 7.10 corresponding to Case (ii) actually requires to perform additional manipulations. At the end of this section, we add a few words about Proposition 7.18.

By 2.18c the equation 2.21 reads

$$
M_{\varepsilon}\left(q_{\varepsilon}\right) q_{\varepsilon}^{\prime \prime}=\gamma^{2} E_{\varepsilon}\left(q_{\varepsilon}\right)+\gamma q_{\varepsilon}^{\prime} \times B_{\varepsilon}\left(q_{\varepsilon}\right)-\left\langle\Gamma_{\varepsilon}\left(q_{\varepsilon}\right), q_{\varepsilon}^{\prime}, q_{\varepsilon}^{\prime}\right\rangle .
$$

The proof now consists in substituting the previous expansions of $\Gamma_{\varepsilon}, E_{\varepsilon}$ and $B_{\varepsilon}$ into the right hand side of $(9.58)$ and to rely on some crucial cancellations. Let $\delta>0$. Using the decomposition 2.28 of $\Gamma$, the definition 4.29$)$ of $p_{\varepsilon}$, the expansions 9.2 and $(9.6)$ for the Christoffel symbols, (9.17) for the electric field, 9.46) for the magnetic field and the relation 9.47) we obtain that, for $\varepsilon_{0}$ in $(0,1)$ small enough, as long as $\left(\varepsilon, q_{\varepsilon}\right)$ belongs to $\mathfrak{Q}_{\delta, \varepsilon_{0}}$ :

$$
\begin{gathered}
\gamma^{2} E_{\varepsilon}\left(q_{\varepsilon}\right)+\gamma q_{\varepsilon}^{\prime} \times B_{\varepsilon}\left(q_{\varepsilon}\right)-\left\langle\Gamma_{\varepsilon}\left(q_{\varepsilon}\right), q_{\varepsilon}^{\prime}, q_{\varepsilon}^{\prime}\right\rangle=I_{\varepsilon}\left[\left(\gamma^{2} \mathrm{E}_{0}\left(q_{\varepsilon}\right)+\gamma p_{\varepsilon} \times B_{\vartheta_{\varepsilon}}^{\mathcal{S}_{0}}\right)\right. \\
\left.+\varepsilon\left(\gamma^{2} \mathrm{E}_{1}\left(q_{\varepsilon}\right)+\gamma p_{\varepsilon} \times \mathrm{B}_{1}\left(q_{\varepsilon}\right)-\left\langle\Gamma_{\vartheta_{\varepsilon}}^{\mathcal{S}_{0}}, p_{\varepsilon}, p_{\varepsilon}\right\rangle\right)+\varepsilon^{2} \check{F}_{r}\left(\varepsilon, q_{\varepsilon}, p_{\varepsilon}\right)\right],
\end{gathered}
$$

where

$$
\begin{aligned}
\check{F}_{r}\left(\varepsilon, q_{\varepsilon}, p_{\varepsilon}\right)=\gamma^{2} E_{r}\left(\varepsilon, q_{\varepsilon}\right)+\gamma p_{\varepsilon} \times B_{r}\left(\varepsilon, q_{\varepsilon}\right)-\varepsilon\left\langle\Gamma_{r}^{\mathrm{rot}}\left(\varepsilon, q_{\varepsilon}\right), p_{\varepsilon}, p_{\varepsilon}\right\rangle & \\
& -\varepsilon\left\langle\Gamma_{r}^{\partial \Omega}\left(\varepsilon, q_{\varepsilon}\right), p_{\varepsilon}, p_{\varepsilon}\right\rangle .
\end{aligned}
$$


Then recalling that $F_{\vartheta_{\varepsilon}}^{\mathcal{S}_{0}}$ is defined in 4.14 , we observe that the zero order term in the right hand side of 9.59 (in terms of powers of $\varepsilon$ ) can be recast as follows:

$$
\gamma^{2} \mathrm{E}_{0}\left(q_{\varepsilon}\right)+\gamma p_{\varepsilon} \times B_{\vartheta_{\varepsilon}}^{\mathcal{S}_{0}}\left(q_{\varepsilon}\right)=F_{\vartheta_{\varepsilon}}^{\mathcal{S}_{0}}\left(\varepsilon \vartheta_{\varepsilon}^{\prime}, h_{\varepsilon}^{\prime}-\gamma u^{\Omega}\left(h_{\varepsilon}\right)\right) .
$$

Now, in order to deal with the subprincipal term of the right hand side of (9.59), let us state the following crucial lemma, where we consider only the part $\mathrm{E}_{1}^{a}\left(q_{\varepsilon}\right)$ defined in 9.12 of the decomposition 9.7$)$ of the term $\mathrm{E}_{1}\left(q_{\varepsilon}\right)$.

Lemma 9.9. The following holds:

$$
\gamma^{2} \mathrm{E}_{1}^{a}\left(q_{\varepsilon}\right)+\gamma p_{\varepsilon} \times \mathrm{B}_{1}\left(q_{\varepsilon}\right)-\left\langle\Gamma_{\vartheta}^{\mathcal{S}_{0}}, p_{\varepsilon}, p_{\varepsilon}\right\rangle=-\left\langle\Gamma_{\vartheta}^{\mathcal{S}_{0}}, \hat{p}_{\varepsilon}, \hat{p}_{\varepsilon}\right\rangle,
$$

where

$$
\hat{p}_{\varepsilon}:=\left(\varepsilon \vartheta_{\varepsilon}^{\prime}, h_{\varepsilon}^{\prime}-\gamma u^{\Omega}\left(h_{\varepsilon}\right)\right)^{t} .
$$

Remark 9.10. As for 9.60), this relation is algebraic, in the sense that it does not rely on $p_{\varepsilon}=I_{\varepsilon} q_{\varepsilon}^{\prime}$ or on the fact that $q_{\varepsilon}$ satisfies (2.21).

Proof of Lemma 9.9. We will recast the second and third terms of the left hand side in terms of the matrix $M^{\dagger}$ defined in (9.8). Let us start with the Christoffel term. Using the definition of $M_{a, \vartheta}^{\mathcal{S}_{0}}$ in 4.10 and the decomposition of $M_{a}^{\mathcal{S}_{0}}$ in 4.25, we arrive at

$$
M_{a, \vartheta}^{\mathcal{S}_{0}}=\left(\begin{array}{cc}
m^{\#} & \mu_{\vartheta}^{t} \\
\mu_{\vartheta} & M_{b, \vartheta}^{\mathcal{S}_{0}}
\end{array}\right)
$$

with $M_{b, \vartheta}^{\mathcal{S}_{0}}$ and $\mu_{\vartheta}$ as in 9.11. In particular we infer from 4.12 that

$$
\left\langle\Gamma_{\vartheta}^{\mathcal{S}_{0}}, p_{\varepsilon}, p_{\varepsilon}\right\rangle=\left(\begin{array}{c}
-\left(M_{b, \vartheta_{\varepsilon}}^{\mathcal{S}_{0}} h_{\varepsilon}^{\prime}\right)^{\perp} \cdot h_{\varepsilon}^{\prime} \\
\left(\varepsilon \vartheta_{\varepsilon}^{\prime}\right)^{2} \mu_{\vartheta_{\varepsilon}}^{\perp}+\varepsilon \vartheta_{\varepsilon}^{\prime}\left(\left(M_{b, \vartheta_{\varepsilon}}^{\mathcal{S}_{0}} h_{\varepsilon}^{\prime}\right)^{\perp}-M_{b, \vartheta_{\varepsilon}}^{\mathcal{S}_{0}}\left(h_{\varepsilon}^{\prime}\right)^{\perp}\right)
\end{array}\right) .
$$

It remains to recast this expression thanks to the matrix $M^{\dagger}$ defined in (9.8). This is done thanks to the following elementary identities: recalling 9.9 we have for any $\vartheta$ in $\mathbb{R}$ and $X$ in $\mathbb{R}^{2}$,

$$
\begin{aligned}
\left(M_{b, \vartheta}^{\mathcal{S}_{0}} X\right)^{\perp} \cdot X & =X^{\perp} \cdot M_{\vartheta}^{\dagger} X^{\perp}, \\
\left(M_{\mathrm{b}, \vartheta}^{\mathcal{S}_{0}} X\right)^{\perp}-M_{\mathrm{b}, \vartheta}^{\mathcal{S}_{0}} X^{\perp} & =-2 M_{\vartheta}^{\dagger} X, \\
(\perp) M_{\vartheta}^{\dagger}(\perp) & =M_{\vartheta}^{\dagger} .
\end{aligned}
$$

Therefore

$$
\left\langle\Gamma_{\vartheta}^{\mathcal{S}_{0}}, p_{\varepsilon}, p_{\varepsilon}\right\rangle=\left(\begin{array}{c}
-\left(h_{\varepsilon}^{\prime}\right)^{\perp} \cdot M_{\vartheta}^{\dagger}\left(h_{\varepsilon}^{\prime}\right)^{\perp} \\
\left(\varepsilon \vartheta_{\varepsilon}^{\prime}\right)^{2} \mu_{\vartheta_{\varepsilon}}^{\perp}-2 \varepsilon \vartheta_{\varepsilon}^{\prime} M_{\vartheta}^{\dagger} h_{\varepsilon}^{\prime}
\end{array}\right) .
$$

Now using 9.45, 9.66 and the fact that for any $\vartheta$ in $\mathbb{R}, M_{\vartheta}^{\dagger}$ is symmetric, we obtain

$$
p_{\varepsilon} \times \mathrm{B}_{1}\left(q_{\varepsilon}\right)=\left(\begin{array}{c}
-2\left(h_{\varepsilon}^{\prime}\right)^{\perp} \cdot M_{\vartheta_{\varepsilon}}^{\dagger} u^{\Omega}\left(h_{\varepsilon}\right)^{\perp} \\
-2\left(\varepsilon \vartheta_{\varepsilon}^{\prime}\right)^{2}\left(M_{\vartheta_{\varepsilon}}^{\dagger} u^{\Omega}\left(h_{\varepsilon}\right)^{\perp}\right)^{\perp}
\end{array}\right)=\left(\begin{array}{c}
-2\left(h_{\varepsilon}^{\prime}\right)^{\perp} \cdot M_{\vartheta_{\varepsilon}}^{\dagger} u^{\Omega}\left(h_{\varepsilon}\right)^{\perp} \\
-2\left(\varepsilon \vartheta_{\varepsilon}^{\prime}\right)^{2} M_{\vartheta_{\varepsilon}}^{\dagger} u^{\Omega}\left(h_{\varepsilon}\right)
\end{array}\right) .
$$

Now it suffices to combine 9.68, 9.67) and 9.12 to deduce 9.61). 
As a consequence, combining $9.59,9.60$ and 9.61 , we arrive at

$$
\begin{aligned}
& \gamma^{2} E_{\varepsilon}\left(q_{\varepsilon}\right)+\gamma q_{\varepsilon}^{\prime} \times B_{\varepsilon}\left(q_{\varepsilon}\right)-\left\langle\Gamma_{\varepsilon}\left(q_{\varepsilon}\right), q_{\varepsilon}^{\prime}, q_{\varepsilon}^{\prime}\right\rangle \\
= & I_{\varepsilon}\left[F_{\vartheta}^{\mathcal{S}_{0}}\left(\hat{p}_{\varepsilon}\right)+\varepsilon\left\{-\left\langle\Gamma_{\vartheta}^{\mathcal{S}_{0}}, \hat{p}_{\varepsilon}, \hat{p}_{\varepsilon}\right\rangle+\gamma^{2}\left(\mathrm{E}_{1}^{b}\left(q_{\varepsilon}\right)+\mathrm{E}_{1}^{c}\left(q_{\varepsilon}\right)\right)\right\}+\varepsilon^{2} F_{r}\left(\varepsilon, q_{\varepsilon}, \hat{p}_{\varepsilon}\right)\right] .
\end{aligned}
$$

Moreover $F_{r}$ belongs to $L_{\text {loc }}^{\infty}\left(\mathfrak{Q}_{\delta, \varepsilon_{0}} \times \mathbb{R}^{3} ; \mathbb{R}^{3}\right)$, depends on $\mathcal{S}_{0}, \gamma$ and $\Omega$ and is weakly nonlinear in the sense of Definition 6.2. Next the part $\mathrm{E}_{1}^{c}$ of the subprincipal term in 9.69 can be absorbed by the principal term up to a modification of size $\varepsilon$ of the arguments (that is, thanks to the second oder modulation). More precisely, by 4.16 and (9.16),

$$
F_{\mathcal{S}_{0}, \vartheta_{\varepsilon}}\left(\tilde{p}_{\varepsilon}\right)=F_{\mathcal{S}_{0}, \vartheta_{\varepsilon}}\left(\hat{p}_{\varepsilon}\right)+\varepsilon \gamma^{2} \mathrm{E}_{1}^{c}\left(q_{\varepsilon}\right),
$$

where $\tilde{p}_{\varepsilon}$ is given by $(7.8)$. Thus we deduce from $(9.69)$ and 9.70 that

$$
\begin{aligned}
\gamma^{2} E_{\varepsilon}\left(q_{\varepsilon}\right) & +\gamma q_{\varepsilon}^{\prime} \times B_{\varepsilon}\left(q_{\varepsilon}\right)-\left\langle\Gamma_{\varepsilon}\left(q_{\varepsilon}\right), q_{\varepsilon}^{\prime}, q_{\varepsilon}^{\prime}\right\rangle \\
& =I_{\varepsilon}\left[F_{\vartheta_{\varepsilon}}^{\mathcal{S}_{0}}\left(\tilde{p}_{\varepsilon}\right)-\varepsilon\left\langle\Gamma_{\vartheta_{\varepsilon}}^{\mathcal{S}_{0}}, \tilde{p}_{\varepsilon}, \tilde{p}_{\varepsilon}\right\rangle+\varepsilon \gamma^{2} E_{1}^{b}\left(q_{\varepsilon}\right)+\varepsilon^{2} \hat{F}_{r}\left(\varepsilon, q_{\varepsilon}, \tilde{p}_{\varepsilon}\right)\right],
\end{aligned}
$$

where the term $\hat{F}_{r}$ is defined by

$$
\begin{aligned}
\hat{F}_{r}\left(\varepsilon, q_{\varepsilon}, \tilde{p}_{\varepsilon}\right):=\check{F}_{r}\left(\varepsilon, q_{\varepsilon}, \tilde{p}_{\varepsilon}+\varepsilon \gamma p_{c}\left(q_{\varepsilon}\right)\right)-2 \gamma\left\langle\Gamma_{\vartheta_{\varepsilon}}^{\mathcal{S}_{0}}, \tilde{p}_{\varepsilon}, p_{c}\left(q_{\varepsilon}\right)\right\rangle & \\
& -\varepsilon \gamma^{2}\left\langle\Gamma_{\vartheta_{\varepsilon}}^{\mathcal{S}_{0}}, p_{c}\left(q_{\varepsilon}\right), p_{c}\left(q_{\varepsilon}\right)\right\rangle
\end{aligned}
$$

where $p_{c}\left(q_{\varepsilon}\right):=\left(0, u_{c}\left(q_{\varepsilon}\right)\right)$. One can easily check that $\hat{F}_{r}$ is still weakly nonlinear.

Using Proposition 9.1 and 9.71, and recalling the notation 4.33, we recast the equation 9.58 as follows:

$$
\begin{array}{r}
\varepsilon^{\min (2, \alpha)}\left(M_{\vartheta_{\varepsilon}}(\varepsilon)+\varepsilon^{4-\min (2, \alpha)} M_{r}\left(\varepsilon, q_{\varepsilon}\right)\right) p_{\varepsilon}^{\prime}=F_{\vartheta_{\varepsilon}}^{\mathcal{S}_{0}}\left(\tilde{p}_{\varepsilon}\right)-\varepsilon\left\langle\Gamma_{\vartheta_{\varepsilon}}^{\mathcal{S}_{0}}, \tilde{p}_{\varepsilon}, \tilde{p}_{\varepsilon}\right\rangle \\
+\varepsilon \gamma^{2} \mathrm{E}_{1}^{b}\left(q_{\varepsilon}\right)+\varepsilon^{2} \hat{F}_{r}\left(\varepsilon, q_{\varepsilon}, \tilde{p}_{\varepsilon}\right) .
\end{array}
$$

We need to perform further modifications on this equation in order to achieve the normal forms (6.7)- 7.10 exactly, due to the fact that the mass matrix in 9.72 contains some extra lower-order terms, and that the time derivative is applied to $p_{\varepsilon}$ rather than to $\tilde{p}_{\varepsilon}$. To deal with the first discrepancy, reducing $\varepsilon_{0}$ in $(0,1)$ if necessary, we simply multiply 9.72 by the matrix

$$
M_{\vartheta}(\varepsilon)\left(M_{\vartheta}(\varepsilon)+\varepsilon^{4-\min (2, \alpha)} M_{r}(\varepsilon, q)\right)^{-1} .
$$

On the other hand, for the second discrepancy, we observe that the modulated translation velocity $\tilde{\ell}_{\varepsilon}:=h^{\prime}-\gamma\left(u^{\Omega}\left(h_{\varepsilon}\right)+\varepsilon u_{c}\left(q_{\varepsilon}\right)\right)$ satisfies

$$
\begin{aligned}
\tilde{\ell}_{\varepsilon}^{\prime}=h_{\varepsilon}^{\prime \prime}-\gamma \tilde{\ell}_{\varepsilon} & \cdot\left(\nabla u^{\Omega}\right)\left(h_{\varepsilon}\right)-\gamma^{2}\left(u^{\Omega}\left(h_{\varepsilon}\right)+\varepsilon u_{c}\left(q_{\varepsilon}\right)\right) \cdot \nabla u^{\Omega}\left(h_{\varepsilon}\right) \\
& -\gamma D_{\vartheta} u_{c}\left(q_{\varepsilon}\right) \varepsilon \vartheta_{\varepsilon}^{\prime}-\varepsilon \gamma D_{h} u_{c}\left(q_{\varepsilon}\right) \cdot\left(\tilde{\ell}_{\varepsilon}+\gamma\left(u^{\Omega}\left(h_{\varepsilon}\right)+\varepsilon u_{c}\left(q_{\varepsilon}\right)\right)\right) .
\end{aligned}
$$


Thus we obtain 7.10 with $F_{r}$ in $L_{\text {loc }}^{\infty}\left(\mathfrak{Q}_{\delta, \varepsilon_{0}} \times \mathbb{R}^{3} ; \mathbb{R}^{3}\right)$ weakly nonlinear in the sense of Definition 6.2. This ends the proof of Proposition 7.10 .

Starting from 2.35a, the proof of Proposition 7.18 is similar to the one of Proposition 7.10 with some simplifications, since in this case $\tilde{M}_{\mathrm{b}}^{\mathcal{S}_{0}}=\pi \mathrm{Id}_{2}$ and consequently $M^{\dagger}=0$. It follows that $\mathrm{E}_{1}^{a}=0$ and $\mathrm{B}_{1}=0$.

Now we expand $\tilde{M}_{b, \varepsilon}, \Gamma_{b, \varepsilon}, E_{b, \varepsilon}$ and $\tilde{B}_{1, \varepsilon}$ (which depend merely on $h_{c}$ and $\varepsilon$ ) in terms of $\varepsilon$. Note that the last two coordinates of $E_{1}^{b}$ (which are the only ones to be relevant here, recall (2.33)) are zero and that $\mathrm{E}_{1}^{c}$ gives the term $-u_{c}(q)^{\perp}$. Noting that $u^{\Omega}(h)+\varepsilon u_{c}(q)=u^{\Omega}\left(h_{c}\right)+O\left(\varepsilon^{2}\right)$ and recalling (2.34) we infer

$$
E_{\mathrm{b}, \varepsilon}=-u^{\Omega}\left(h_{c}\right)^{\perp}+\varepsilon^{2} E_{\mathrm{b}, r} \text { and } \tilde{B}_{1, \varepsilon}=-1+\varepsilon^{2} B_{\mathrm{b}, r},
$$

with $E_{\mathrm{b}, r}=E_{\mathrm{b}, r}\left(\varepsilon, h_{c, \varepsilon}\right)$ weakly nonlinear in the sense of Definition 7.17 and $B_{b, r}=B_{b, r}\left(\varepsilon, h_{c, \varepsilon}\right)$ bounded as long as $h_{c, \varepsilon}$ is away from $\partial \Omega$. On the other side, one finds that $\tilde{M}_{b}=\varepsilon^{2} \pi \mathrm{Id}_{2}+O\left(\varepsilon^{4}\right)$ and $\Gamma_{b, \varepsilon}=O\left(\varepsilon^{4}\right)$. The conclusion follows easily and this ends the proof of Proposition 7.18

Acknowledgements The authors thank the Agence Nationale de la Recherche, Project IFSMACS, grant ANR-15-CE40-0010 for a partial financial support. The first and third authors were also partially supported by the Agence Nationale de la Recherche, Project DYFICOLTI, grant ANR-13-BS01-0003-01, the second author by the Agence Nationale de la Recherche, Project OPTIFORM, grant ANR-12-BS01-0007-04 and the third author by the Agence Nationale de la Recherche, Project BORDS, grant ANR-16-CE40-0027-01. The third author was also partially supported by the Emergences Project "Instabilities in Hydrodynamics" funded by the Mairie de Paris and the Fondation Sciences Mathématiques de Paris.

\section{References}

1. Arnold, V. I., Kozlov, V. V.; Neishtadt, A. I., Mathematical aspects of classical and celestial mechanics. [Dynamical systems. III]. Translated from the Russian original by E. Khukhro. Third edition. Encyclopaedia of Mathematical Sciences, 3. Springer-Verlag, Berlin, 2006.

2. Berkowitz J., Gardner C. S., On the asymptotic series expansion of the motion of a charged particle in slowly varying fields. Comm. Pure Appl. Math. 12 (1959), 501-512.

3. Bonnaillie-Noël V., Dambrine M., Tordeux S., Vial G., Interactions between moderately close inclusions for the Laplace equation. M3AS: Mathematical Models and Methods in Applied Sciences, 19 (2009), no. 10, 1853-1882.

4. Brenier Y., Convergence of the Vlasov-Poisson system to the incompressible Euler equations. Comm. Partial Differential Equations 25 (2000), no. 3-4, 737-754.

5. Cardone G., Nazarov S.A., Sokolowski J., Asymptotics of solutions of the Neumann problem in a domain with closely posed components of the boundary. Asymptotic Analysis 62 (2009), 41-88.

6. Chambrion T., Munnier A., Generic controllability of $3 \mathrm{~d}$ swimmers in a perfect fluid. SIAM Journal on Control and Optimization, 50(5) (2012), 2814-2835.

7. Folland G. B., Introduction to partial differential equations. Second edition. Princeton University Press, Princeton, NJ, 1995.

8. Friedrichs K. O., Special topics in fluid dynamics, Gordon and Breach, New York, 1966.

9. Gallay T., Interaction of vortices in weakly viscous planar flows. Arch. Ration. Mech. Anal. 200 (2011), no. 2, 445-490. 
10. Glass O., Kolumbán J., Sueur F. External boundary control of the motion of a rigid body immersed in a perfect two-dimensional fluid. arXiv preprint (2017), arXiv:1707.05093.

11. Glass O., Lacave C., Sueur F., On the motion of a small body immersed in a two dimensional incompressible perfect fluid. Bull. Soc. Math. France. 142 (2014), no 3, 489-536.

12. Glass O., Lacave C., Sueur F., On the motion of a small light body immersed in a two dimensional incompressible perfect fluid. Communications in Math. Physics. 341 (2016), Issue 3, 1015-1065.

13. Glass O., Sueur F., The movement of a solid in an incompressible perfect fluid as a geodesic flow. Proc. Amer. Math. Soc. 140 (2012), no. 6, 2155-2168.

14. Glass O., Sueur F., Uniqueness results for weak solutions of two-dimensional fluid-solid systems. Arch. Ration. Mech. Anal. 218 (2105), no. 2, 907-944.

15. Glass O., Sueur F., On the motion of a rigid body in a two-dimensional irregular ideal flow. SIAM J. Math. Anal. 44 (2012), no. 5, 3101-3126.

16. Helmholtz H., Über Integrale der hydrodynamischen Gleichungen, welche den Wirbelbewegungen entsprechen, Crelles J. 55, 25 (1858). Translation in: On the integral of the hydrodynamical equations which express vortex motion, Phil. Mag. 33, 485-513 (1867).

17. Henrot A., Pierre M., Variation et Optimisation de Formes. Une Analysis Géométrique, Mathématiques and Applications, Vol. 48, Springer-Verlag, Berlin, 2005.

18. Houot J., Munnier A., On the motion and collisions of rigid bodies in an ideal fluid. Asymptot. Anal. 56 (2008), no. 3-4, 125-158.

19. Iftimie D., Lopes Filho M. C., Nussenzveig Lopes H. J., Two dimensional incompressible ideal flow around a small obstacle. Comm. Partial Differential Equations 28 (2003), no. $1-2,349-379$.

20. Il'in A. M., Matching of asymptotic expansions of solutions of boundary value problems. Translated from the Russian by V. Minachin. Translations of Mathematical Monographs, 102. American Mathematical Society, Providence, RI, 1992.

21. Kelvin W. Thomson, Lord $\sim$, Mathematical and Physical Papers. Cambridge University Press, Cambridge, UK, 1910.

22. Kirchhoff G., Vorlesungen über mathematische Physik, Mechanik. Teuber, Leipzig, 1876.

23. Lamb H., Hydrodynamics. Reprint of the 1932 sixth edition. Cambridge University Press, 1993.

24. Lin C. C., On the motion of vortices in two dimensions I. Existence of the KirchhoffRouth function. Proc. Natl. Acad. Sci. U.S.A. 27 (1941), 570-575.

25. Lin C. C., On the motion of vortices in two dimensions II. Some further investigations on the Kirchhoff-Routh function. Proc. Natl. Acad. Sci. U.S.A. 27 (1941), 575-577.

26. Lopes Filho M. C., Vortex dynamics in a two-dimensional domain with holes and the small obstacle limit. SIAM J. Math. Anal. 39 (2007), no. 2, 422-436.

27. Marchioro C., Pulvirenti M., Mathematical theory of incompressible nonviscous fluids. Applied Mathematical Sciences 96, Springer-Verlag, New York, 1994.

28. McLean W., Strongly elliptic systems and boundary integral equations. Cambridge University Press, Cambridge, 2000.

29. Maz'ya V., Nazarov S., Plamenevskij B., Asymptotic theory of elliptic boundary value problems in singularly perturbed domains. Vol. I. Translated from the German by Georg Heinig and Christian Posthoff. Operator Theory: Advances and Applications, 111. Birkhuser Verlag, Basel, 2000.

30. Milne-Thomson L. M., Theoretical hydrodynamics. 4th ed. The Macmillan Co., New York, 1960.

31. Moussa A., Sueur F., A 2d spray model with gyroscopic effects. Asymptotic analysis. Volume 81 (2013), Issue 1, 53-91.

32. Munnier, A. On the self-displacement of deformable bodies in a potential fluid flow. Mathematical Models and Methods in Applied Sciences, 18:11 (2008), 1945-1981.

33. Munnier A., Locomotion of Deformable Bodies in an Ideal Fluid: Newtonian versus Lagrangian Formalisms. J. Nonlinear Sci (2009) 19: 665-715.

34. Munnier A., Ramdani K., Asymptotic analysis of a Neumann problem in a domain with cusp. Application to the collision problem of rigid bodies in a perfect fluid. SIAM Journal on Mathematical Analysis, 47 (2015), no. 6, 4360-4403.

35. Newton P.K., The N-Vortex Problem: Analytical Techniques, Applied Mathematical Sciences Series, Vol. 145, Springer-Verlag, New York, 2001. 
36. Poincaré H., Théorie des tourbillons. George Carré, Paris, 1893.

37. Pommerenke C., Univalent functions. With a chapter on quadratic differentials by Gerd Jensen. Studia Mathematica/Mathematische Lehrbücher, Band XXV. Vandenhoeck and Ruprecht, Göttingen, 1975.

38. Reynolds O., Papers on Mechanical and Physical Subjects, the Sub-Mechanics of the Universe, volume 3. Cambridge University Press, Cambridge, 1903.

39. Routh E. J., Some applications of conjugate functions. Proc. Lond. Math. Soc. 12 (1881), 73-89.

40. Sokołowski J., Zolésio J.-P., Introduction to shape optimization. Shape sensitivity analysis. Springer Series in Computational Mathematics, 16, Springer-Verlag, Berlin, 1992.

41. Turkington B., On the evolution of a concentrated vortex in an ideal fluid. Arch. Rational Mech. Anal. 97 (1987), no. 1, 75-87.

42. Vankerschaver J., Kanso E., Marsden. J. E. The Geometry and Dynamics of Interacting Rigid Bodies and Point Vortices. J. Geom. Mech. 1 (2009), no. 2, 223-266. 